\title{
Psychological adjustment to stress and trauma : hippocampal configuration learning, cognitive emotion regulation, and frontal brain asymmetry, as predictors of resilience
}

Citation for published version (APA):

Meyer, T. (2015). Psychological adjustment to stress and trauma : hippocampal configuration learning, cognitive emotion regulation, and frontal brain asymmetry, as predictors of resilience. [Doctoral Thesis, Maastricht University]. Maastricht University. https://doi.org/10.26481/dis.20150423tm

Document status and date:

Published: 01/01/2015

DOI:

10.26481/dis.20150423tm

Document Version:

Publisher's PDF, also known as Version of record

Please check the document version of this publication:

- A submitted manuscript is the version of the article upon submission and before peer-review. There can be important differences between the submitted version and the official published version of record. People interested in the research are advised to contact the author for the final version of the publication, or visit the DOI to the publisher's website.

- The final author version and the galley proof are versions of the publication after peer review.

- The final published version features the final layout of the paper including the volume, issue and page numbers.

Link to publication

\footnotetext{
General rights rights.

- You may freely distribute the URL identifying the publication in the public portal. please follow below link for the End User Agreement:

www.umlib.nl/taverne-license

Take down policy

If you believe that this document breaches copyright please contact us at:

repository@maastrichtuniversity.nl

providing details and we will investigate your claim.
}

Copyright and moral rights for the publications made accessible in the public portal are retained by the authors and/or other copyright owners and it is a condition of accessing publications that users recognise and abide by the legal requirements associated with these

- Users may download and print one copy of any publication from the public portal for the purpose of private study or research.

- You may not further distribute the material or use it for any profit-making activity or commercial gain

If the publication is distributed under the terms of Article 25fa of the Dutch Copyright Act, indicated by the "Taverne" license above,

Download date: 26 Apr. 2023 


\section{Psychological adjustment to stress and trauma}

Hippocampal configuration learning, cognitive emotion regulation, and frontal brain asymmetry, as predictors of resilience

Thomas Meyer 
Printing: $\quad$ Ridderprint BV

Cover: $\quad$ Robert Kanters, Ridderprint BV

Photograph: Maciej Bledowski

Grove of Oddly Shaped Pine Trees in Crooked Forest, Poland.

Copyright (C) by Thomas Meyer, Maastricht 2015

ISBN 978-94-6299-070-8 


\section{Psychological adjustment to stress and trauma}

Hippocampal configuration learning, cognitive emotion regulation, and frontal brain asymmetry, as predictors of resilience

\section{DISSERTATION}

to obtain the degree of Doctor at Maastricht University, on the authority of the Rector Magnificus, Prof. Dr. L.L.G. Soete

in accordance with the decision of the Board of Deans,

to be defended in public

on Thursday, $23^{\text {rd }}$ of April 2015 at 14.00 hours

by

Thomas Meyer 


\section{Supervisor}

Prof. dr. Harald Merckelbach

\section{Co-supervisors}

Dr. Tom Smeets

Dr. Timo Giesbrecht

\section{Assessment Committee}

Prof. dr. A. Jansen (chair)

Prof. dr. A. Arntz (Universiteit van Amsterdam)

Prof. dr. D. Bernstein

Prof. dr. I. Engelhard (Universiteit Utrecht)

Dr. M. Jelicic 


\section{Contents}

$\begin{array}{lll}\text { Chapter } 1 & \text { General introduction: Exploring markers of psychological } & 7\end{array}$ adjustment.

Chapter 2 Individual differences in spatial configuration learning predict the occurrence of intrusive memories.

Chapter 3 Acute stress differentially affects spatial configuration learning in high and low cortisol-responding healthy adults.

Chapter 4 The efficiency of reappraisal and expressive suppression in regulating everyday affective experiences.

Chapter 5 Frontal EEG asymmetry as predictor of physiological responses to aversive memories.

Chapter 6 The role of frontal EEG asymmetry in post-traumatic stress disorder.

Chapter 7 General Discussion

Summary

Samenvatting

Valorisation Addendum

Acknowledgments

Curriculum vitae 



\section{Chapter 1}

General introduction:

Exploring markers of psychological adjustment 



\section{Adjustment to potentially traumatic experiences}

At some point in their life, many people are exposed to potentially traumatic events, such as the death of a close friend, life threatening accidents, or being witness to a violent crime (Ozer, Best, Lipsey, \& Weiss, 2003). A negative outcome that may follow the fear, horror, or helplessness experienced during these events is post-traumatic stress disorder (PTSD). Trauma victims who develop PTSD suffer from prolonged reactions to the event, including re-experiencing (e.g., intrusions, nightmares), avoidance of cues and situations related to the trauma, more negative mood and cognitions, and increased general arousal (American Psychiatric Association, 2013). However, only a minority of those who have been exposed to potentially traumatic experiences actually develops PTSD, and most people are able to adapt reasonably well to these adverse experiences. This ability has been coined resilience (Bonanno, 2004, 2012; Bonanno \& Mancini, 2008). In fact, the severity of PTSD symptoms is only modestly related to the objective severity of the traumatic event (Brewin, Andrews, \& Valentine, 2000; Kessler, Sonnega, Bromet, Hughes, \& Nelson, 1995; McNally \& Robinaugh, 2011), and it is now well established that people differ in how they initially respond to trauma, and how they recover from it (Bonanno, 2012). Therefore, researchers and clinicians alike have been intrigued by the question of what determines the course of psychological adjustment following stress and trauma. In other words, why do some people suffer from pathological symptoms after adversity, while others are resilient?

The interest in factors that predict psychological adjustment to trauma prospectively is fuelled by the ambition of eventually understanding and preventing the development of PTSD more effectively. In the past decades, research on the predictive factors of PTSD has advanced rapidly and has provided insights into risk and resilience factors. Importantly, studies suggest that psychological adjustment to trauma is related to individual differences in various psychological and psychophysiological factors before, during, and after the traumatic event (e.g., Marmar et al., 2006; for a meta-analysis, see Ozer et al., 2003). This implies that the development of PTSD may not be attributable to a single mechanism. Rather, it can be best understood as the result of a complex causal chain of antecedents. The following paragraphs highlight two groups of psychological factors that are involved in this disorder and that have received considerable attention in the PTSD literature: disordered memory and emotion regulation. 


\section{Disordered memory}

Among the cognitive factors proposed to precede and fuel PTSD symptomatology are maladaptive encoding, consolidation, and the (re)construction of traumatic memories (Brewin, Dalgleish, \& Joseph, 1996; Ehlers \& Clark, 2000; McNally, 2003; Rubin, Berntsen, $\&$ Bohni, 2008). Our knowledge of these factors is based on the recurrent finding that PTSD is accompanied by various alterations in memory functioning (for a review, see Brewin, 2011), including an impairment in recalling very specific episodic memories (e.g., Wessel, Merckelbach, \& Dekkers, 2002). In line with this, neuroendocrine accounts of PTSD posit that physiological hyper-arousal and hormonal deregulation during a traumatic experience can lead to dysfunctional activity in memory-encoding structures of the brain (e.g., de Quervain, Aerni, Schelling, \& Roozendaal, 2009; Elzinga \& Bremner, 2002). This could acutely or lastingly compromise memory functioning, and eventually lead to the development of PTSD (e.g., Delahanty \& Nugent, 2006; van Wingen, Geuze, Vermetten, \& Fernandez, 2011; but see Jelicic \& Merckelbach, 2004). Notably, accumulating evidence suggests that hormonal and psychophysiological functioning might be abnormal already pre-trauma in individuals who develop PTSD after trauma (i.e., they constitute vulnerability factors; Gilbertson et al., 2007; Inslicht et al., 2011; Pole et al., 2009; van Zuiden et al., 2012). In sum, clinical and neuropsychological theories conceptualize PTSD as a disorder of memory. Thus, individual differences in memory and learning processes could be identified as risk factors for this disorder.

One detailed neuroanatomical model of memory formation during traumatic experiences is the dual representation model of Brewin and colleagues (Brewin et al., 1996; Brewin, Gregory, Lipton, \& Burgess, 2010). According to this model, two distinct but interacting memory systems in the brain are responsible for the occurrence of intrusive memories, a hallmark feature of PTSD. The first system is a sensation-based memory system that encodes perceptual and affective qualities of events in cortical and subcortical sensory areas, including the insula and amygdala. The processing of information within this system purportedly is enhanced during stressful situations. The second system is a context-based memory system that supports further processing of the sensory input both by recruiting sensory association areas and by translating the egocentric viewpoint of the sensory input into abstract, allocentric representations. Only in this abstract form, trauma representations are verbally and voluntarily accessible, and can be integrated with other autobiographical memories. The brain structures involved in context-based memory are the hippocampus and parahippocampus. This model 
predicts that intrusions are more likely to occur when highly emotional sensation-based memories are formed in the absence of a complete context-based memory for the traumatic event. In this situation, sensation-based trauma memories can be triggered on their own. As a result, the memory is experienced from an egocentric viewpoint, such that the reactivated affective states are perceived as having immediate significance.

Since its proposition in 1996, the dual representation model of PTSD has had a considerable impact on the trauma memory field. As Figure 1.1 illustrates, the original article by Brewin et al. (1996) continues to be widely referred to in the international scientific literature. However, despite the model's prominence, empirical evaluations of the theory remain surprisingly scarce. Therefore, one aim of this dissertation was to contribute to the empirical foundation of the dual representation model. In particular, we followed the idea to that the formation of allocentric, context-based memory in the hippocampal area reduces, and perhaps even prevents, the occurrence of intrusions after aversive experiences (Brewin et al., 2010). A testable prediction flowing from this model is that individuals with a superior context-based memory system should be more resilient after exposure to potentially traumatic events. This idea inspired part of this dissertation and will be addressed in more detail in later sections.

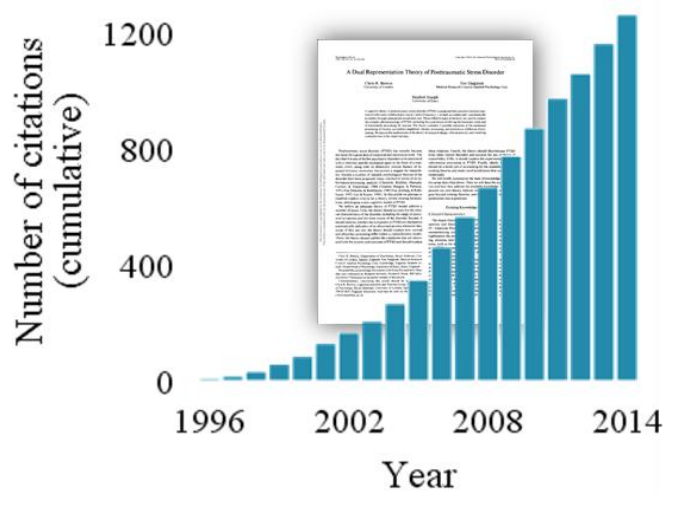

Figure 1.1. Cumulative number of citations per year of the article "A dual representation theory of posttraumatic stress disorder" by Brewin et al. (1996). Source: Google Scholar, 2014. 


\section{Disordered emotions}

While PTSD appears to be characterized by disturbed memory mechanisms, it can also be regarded as an emotional disorder. Usually, emotions are highly adaptive, as they serve to guide human behaviour and help prepare behavioural reactions quickly and in an automatic fashion (Most, Chun, Widders, \& Zald, 2005). This can be essential for survival in a lifethreatening situation. Likewise, strong emotional reactions to cues of past aversive experiences, as the ones that are often displayed by trauma survivors, help to prevent the risk of being exposed to similar situations again. However, situational demands can change and it may become inadequate to express or use specific emotions as a guide for behaviour. To this end, healthy humans are able to alter the strength and direction of their emotional responses by employing emotion regulation (Thompson, 1991). In patients with PTSD or other emotional disorders, this ability may be impaired (Aldao \& Nolen-Hoeksema, 2010; Berking et al., 2008). The literature describes several distinctive cognitive and behavioural tendencies in these patients, including biased information processing, maladaptive beliefs, and the way an individual responds to the reoccurrence of trauma memories (Brewin \& Holmes, 2003; Engelhard, Macklin, McNally, van den Hout, \& Arntz, 2001; Thrasher, Dalgleish, \& Yule, 1994). These tendencies may explain why PTSD patients are unable to recover from a traumatic provocation, and why their emotional reactions remain rigid and exaggerated, even if the environmental context is not dangerous any longer (Aldao, 2013). Thus, emotion regulation can have profound effects on emotional well-being and may determine the persistence of symptoms long after the traumatic event (Dunmore, Clark, \& Ehlers, 2001; Ehlers, 2010; Ehlers, Mayou, \& Bryant, 1998; Engelhard et al., 2001).

Since deficits in the ability to regulate emotions can hinder adjustment to shocking experiences, one can expect them to predict the risk of developing PTSD symptoms. And indeed, clinical research indicates that specific emotion regulation skills play a key role in psychological adjustment and emotional disorders (Berking et al., 2008; Garnefski et al., 2002). An emotion regulation strategy that is thought to be particularly relevant for well-being is called cognitive reappraisal. This strategy entails changing cognitions about a situation in order to alter the emotions it elicits. A second strategy that has received considerable attention in research is expressive suppression, which refers to counteracting bodily responses and response tendencies that are activated once an emotion has been fully generated (Gross \& Thompson, 2007). Individuals who use cognitive reappraisal more and expressive suppression less frequently have often been found to have lower levels of depression, anxiety, and stress- 
related symptoms (e.g., Amstadter, 2008; Joormann \& Gotlib, 2010; Moore, Zoellner, \& Mollenholt, 2008). Thus, the relative use of these emotion regulation strategies may serve as a possible indicator of how well an individual can psychologically adjust to a traumatic experience.

The degree to which individuals habitually employ reappraisal and suppression can be assessed using self-report instruments, the most widely known example being the Emotion Regulation Questionnaire (ERQ; Gross \& John, 2003). A particular advantage of self-report measures is that they are easy to use and interpret, and can thus have a great utility in clinical practice. However, the use of self-report relies critically on the assumptions that respondents collaborate benevolently with the experimenter (or practitioner), and that individuals actually have an accurate insight in their own cognitive and emotional processes that are relevant to resilience. While these assumptions may not always be correct, another potential problem is that self-report measures are often subject to cognitive or memory biases (Schwarz, 2012). Therefore, there are practical and theoretical interests to find more objective markers of emotion regulation and psychological adjustment in addition to self-report measures, such as hormone levels or psychophysiological activity.

This dissertation devotes particular attention to a biomarker called frontal asymmetry, as one potential objective indicator of resilience. It refers to a difference in mean alpha band power (typically 8-13 Hz) between the left and right frontal cortex over a time span of several minutes, and is usually measured using electroencephalography (EEG; Coan \& Allen, 2003). Based on the assumption that alpha band power is indicative of brain idling (Pfurtscheller, Stancak, \& Neuper, 1996), frontal asymmetry is widely interpreted in terms of hemispheric differences in frontal brain activity. It has been proposed to reflect the relative activity of two lateralized brain systems subserving motivation and emotion, determining the individual's style of affective responding in emotional situations (Davidson, 1998; Heller, 1993).

A large and growing literature suggests an intimate link between frontal asymmetry and emotional responding and regulation. For instance, frontal asymmetry has repeatedly been found to predict emotional and behavioural reactions to stressful situations, as well as the presence of psychopathology. In particular, more left-sided frontal activity at rest has been linked to superior affect and stress regulation (e.g., Koslov, Mendes, Pajtas, \& Pizzagalli, 2011; Tomarken, Davidson, Wheeler, \& Doss, 1992), lower levels of anxiety and depression (Thibodeau, Jorgensen, \& Kim, 2006), and lower risk of developing depression (Nusslock et al., 2011). Therefore, this non-invasive measure has become a promising candidate in the 
search for objective markers of resilience. However, compared with research on markers of depression, relatively few studies have investigated whether frontal asymmetry may be specifically related to the risk of developing PTSD symptoms following aversive experiences. Two sections of this dissertation address this idea in more detail.

\section{Aims of the present dissertation}

With the above-mentioned considerations in mind, the work presented in this dissertation aimed to explore the theoretical and practical relevance of potential predictors of resilience following trauma exposure. Broadly, the predictors that were addressed here can be grouped as memory-related and emotion-related factors (see Figure 1.2). Importantly, all empirical studies that are included here were designed to model resilience in a controlled laboratory environment, relying on healthy volunteers as participants. For instance, to study emotion regulation, mood induction by means of emotional films was employed (see Rottenberg, Ray, \& Gross, 2007), or emotional video fragments served as a laboratory analogue of a potentially traumatic experience (see Weidmann, Conradi, Grögera, Fehma, \& Fydrich, 2009). This latter approach, the so-called trauma-film paradigm (Holmes \& Bourne, 2008), was used to induce negative (involuntary) memories, analogue to intrusions in PTSD patients. An advantage of this methodology is that it allows exploring articulated links between specific individual characteristics and PTSD-analogue outcomes in a controlled environment. We were thus able to control the type and objective intensity of the aversive experience, which is often a problem in studies that include trauma-exposed individuals (e.g., PTSD patients). Furthermore, relatively homogenous and high-functioning samples of participants reduce the influence of (pre-trauma) psychopathology that may overshadow potential effects. However, one should keep in mind that the methodological approach in our studies may limit the generalizability of the findings. Rather than having immediate practical relevance for professionals working with trauma victims, the work described in this dissertation should be regarded as first, but necessary, steps in the exploration of specific markers of resilience. Thus, the current work aims to inform, and serve as an inspiration for, clinical research with trauma victims. In doing so, this work ultimately aims to contribute to the development of cognitive and biological markers of resilience and their clinical application, for instance as screening or diagnostic instruments. 


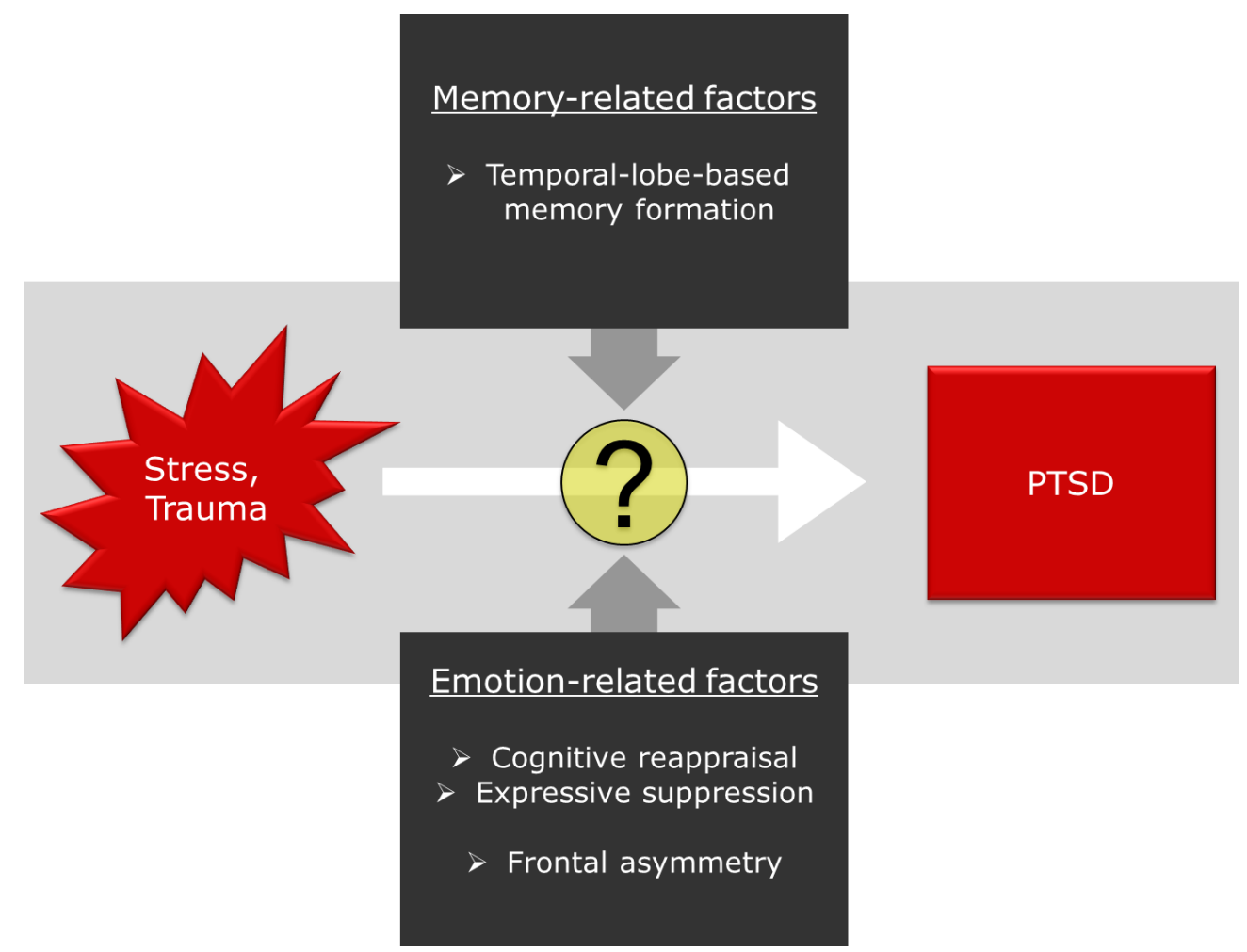

Figure 1.2. Overview of memory- and emotion-related factors investigated in this dissertation. The main aim was to explore whether they moderate psychological adjustment and PTSD symptoms following trauma exposure (grey arrows). As a secondary interest, we also explored how stressful experiences influence memory formation and frontal asymmetry.

\section{Overview of the Chapters}

The specific content of the following chapters is as follows. The first two empirical chapters test specific predictions flowing from the dual-representation model of intrusions, namely that temporal-lobe-based memory formation is related to intrusions following aversive experiences, and that acute stress plays a role in this relationship. In particular, as can be read in Chapter 2, we tested whether participants' performance on an implicit spatial learning task would predict the number of intrusions over the course of one week and physiological responses to reminder cues following exposure to a trauma film. Chapter 3 examines whether the same learning performance changes when participants are acutely stressed. Chapter 4 then turns to emotion regulation, and investigates whether specific emotion regulation strategies are associated with better emotional recovery following a laboratory mood induction on the one hand, and with everyday affect characteristics, on the other hand. The two subsequent chapters address a possible involvement of frontal asymmetry in the emotional adjustment to 
aversive experiences. In particular, Chapter 5 tests the hypothesis that frontal asymmetry might predict physiological self-regulation in response to reactivation of aversive memories. Chapter 6 reviews empirical studies on frontal asymmetry and affective processing, and aims to shed light on the neuropsychological origins of this asymmetry. Furthermore, this chapter discusses the relevance of functional asymmetries in the frontal cortex for our neuropsychological understanding of PTSD, and reviews the available empirical evidence bearing on an involvement of frontal asymmetry in this disorder. Finally, Chapter 7 briefly summarizes the findings of this dissertation, highlights several key theoretical and practical implications, and presents some of our latest, preliminary data. As well, it indicates promising avenues for future research and discusses the importance of bringing together experimental studies with healthy participants and studies with PTSD patients to advance the field. 


\section{References}

Aldao, A. (2013). The future of emotion regulation research: Capturing context. Perspectives on Psychological Science, 8, 155-172. doi: 10.1177/1745691612459518

Aldao, A., \& Nolen-Hoeksema, S. (2010). Specificity of cognitive emotion regulation strategies: A transdiagnostic examination. Behaviour Research and Therapy, 48, 974983. doi: 10.1016/j.brat.2010.06.002

American Psychiatric Association. (2013). Diagnostic and statistical manual of mental disorders (5th ed.). Arlington, VA: American Psychiatric Publishing.

Amstadter, A. (2008). Emotion regulation and anxiety disorders. Journal of Anxiety Disorders, 22, 211-221. doi: 10.1016/j.janxdis.2007.02.004

Berking, M., Wupperman, P., Reichardt, A., Pejic, T., Dippel, A., \& Znoj, H. (2008). Emotion-regulation skills as a treatment target in psychotherapy. Behaviour Research and Therapy, 46, 1230-1237. doi: 10.1016/j.brat.2008.08.005

Bonanno, G. A. (2004). Loss, trauma, and human resilience - Have we underestimated the human capacity to thrive after extremely aversive events? American Psychologist, 59, 20-28. doi: 10.1037/0003-066x.59.1.20

Bonanno, G. A. (2012). Uses and abuses of the resilience construct: Loss, trauma, and healthrelated adversities. Social Science \& Medicine, 74, 753-756. doi: 10.1016/j.socscimed.2011.11.022

Bonanno, G. A., \& Mancini, A. D. (2008). The human capacity to thrive in the face of potential trauma. Pediatrics, 121, 369-375. doi: 10.1542/peds.2007-1648

Brewin, C. R. (2011). The nature and significance of memory disturbance in posttraumatic stress disorder. Annual Review of Clinical Psychology, 7, 203-227. doi:10.1146/annurev-clinpsy-032210-104544

Brewin, C. R., Andrews, B., \& Valentine, J. D. (2000). Meta-analysis of risk factors for posttraumatic stress disorder in trauma-exposed adults. Journal of Consulting and Clinical Psychology, 68, 748-766.

Brewin, C. R., Dalgleish, T., \& Joseph, S. (1996). A dual representation theory of posttraumatic stress disorder. Psychological Review, 103, 670-686.

Brewin, C. R., Gregory, J. D., Lipton, M., \& Burgess, N. (2010). Intrusive images in psychological disorders: Characteristics, neural mechanisms, and treatment implications. Psychological Review, 117, 210-232. doi: 10.1037/a0018113 
Brewin, C. R., \& Holmes, E. A. (2003). Psychological theories of posttraumatic stress disorder. Clinical Psychology Review, 23, 339-376. doi: 10.1016/S02727358(03)00033-3

Coan, J. A., \& Allen, J. J. B. (2003). The state and trait nature of frontal EEG asymmetry in emotion. In K. Hugdahl \& R. J. Davidson (Eds.), The asymmetrical brain (pp. 565615). Cambridge: MIT Press.

Davidson, R. J. (1998). Affective style and affective disorders: Perspectives from affective neuroscience. Cognition \& Emotion, 12, 307-330.

de Quervain, D. J. F., Aerni, A., Schelling, G., \& Roozendaal, B. (2009). Glucocorticoids and the regulation of memory in health and disease. Frontiers in Neuroendocrinology, 30, 358-370. doi: 10.1016/j.yfrne.2009.03.002

Delahanty, D. L., \& Nugent, N. R. (2006). Predicting PTSD prospectively based on prior trauma history and immediate biological responses. In R. Yehuda (Ed.), Psychobiology of Posttraumatic Stress Disorder: A Decade of Progress (Vol. 1071, pp. 27-40). Boston: New York Academy of Science.

Dunmore, E., Clark, D. M., \& Ehlers, A. (2001). A prospective investigation of the role of cognitive factors in persistent posttraumatic stress disorder (PTSD) after physical or sexual assault. Behaviour Research and Therapy, 39, 1063-1084.

Ehlers, A. (2010). Understanding and treating unwanted trauma memories in posttraumatic stress disorder. Zeitschrift Fur Psychologie-Journal of Psychology, 218, 141-145. doi: $10.1027 / 0044-3409 / \mathrm{a} 000021$

Ehlers, A., \& Clark, D. M. (2000). A cognitive model of posttraumatic stress disorder. Behaviour Research and Therapy, 38, 319-345. doi: 10.1016/S0005-7967(99)00123-0

Ehlers, A., Mayou, R. A., \& Bryant, B. (1998). Psychological predictors of chronic posttraumatic stress disorder after motor vehicle accidents. Journal of Abnormal Psychology, 107, 508-519.

Elzinga, B. M., \& Bremner, J. D. (2002). Are the neural substrates of memory the final common pathway in posttraumatic stress disorder (PTSD)? Journal of Affective Disorders, 70, 1-17.

Engelhard, I. M., Macklin, M. L., McNally, R. J., van den Hout, M. A., \& Arntz, A. (2001). Emotion- and intrusion-based reasoning in Vietnam veterans with and without chronic posttraumatic stress disorder. Behaviour Research and Therapy, 39, 1339-1348. doi: 10.1016/S0005-7967(00)00101-7 
Garnefski, N., Van den Kommer, T., Kraaij, V., Teerds, J., Legerstee, J., \& Onstein, E. (2002). The relationship between cognitive emotion regulation strategies and emotional problems: Comparison between a clinical and a non-clinical sample. European Journal of Personality, 16, 403-420. doi: 10.1002/per.458

Gilbertson, M. W., Williston, S. K., Paulus, L. A., Lasko, N. B., Gurvits, T. V., Shenton, M. E., . . O Orr, S. P. (2007). Configural cue performance in identical twins discordant for posttraumatic stress disorder: Theoretical implications for the role of hippocampal function. Biological Psychiatry, 62, 513-520. doi: 10.1016/j.biopsych.2006.12.023

Gross, J. J., \& John, O. P. (2003). Individual differences in two emotion regulation processes: Implications for affect, relationships, and well-being. Journal of Personality and Social Psychology, 85, 348-362. doi: 10.1037/0022-3514.85.2.348

Gross, J. J., \& Thompson, R. A. (2007). Emotion regulation: Conceptual foundations. In J. J. Gross (Ed.), Handbook of emotion regulation (pp. 3-24). New York: The Guilford Press.

Heller, W. (1993). Neuropsychological mechanisms of individual differences in emotion, personality, and arousal. Neuropsychology, 7, 476-489.

Holmes, E. A., \& Bourne, C. (2008). Inducing and modulating intrusive emotional memories: A review of the trauma film paradigm. Acta Psychologica, 127, 553-566. doi: 10.1016/j.actpsy.2007.11.002

Inslicht, S. S., Otte, C., McCaslin, S. E., Apfel, B. A., Henn-Haase, C., Metzler, T., . . . Marmar, C. R. (2011). Cortisol awakening response prospectively predicts peritraumatic and acute stress reactions in police officers. Biological Psychiatry, 70, 1055-1062.

Jelicic, M., \& Merckelbach, H. (2004). Traumatic stress, brain changes, and memory deficits: A critical note. Journal of Nervous and Mental Disease, 192, 548-553. doi: 10.1097/01.nmd.0000135494.20416.59

Joormann, J., \& Gotlib, I. H. (2010). Emotion regulation in depression: Relation to cognitive inhibition. Cognition \& Emotion, 24, 281-298. doi: 10.1080/02699930903407948

Kessler, R. C., Sonnega, A., Bromet, E., Hughes, M., \& Nelson, C. B. (1995). Posttraumatic stress disorder in the National Comorbidity Survey. Archives of General Psychiatry, $52,1048-1060$. 
Koslov, K., Mendes, W. B., Pajtas, P. E., \& Pizzagalli, D. A. (2011). Asymmetry in resting intracortical activity as a buffer to social threat. Psychological Science, 22, 641-649. doi: 10.1177/0956797611403156

Marmar, C. R., McCaslin, S. E., Metzler, T. J., Best, S., Weiss, D. S., Fagan, J., . . Neylan, T. (2006). Predictors of posttraumatic stress in police and other first responders. In R. Yehuda (Ed.), Psychobiology of posttraumatic stress disorder: A decade of progress (pp. 1-18). Boston: New York Academy of Science.

McNally, R. J. (2003). Psychological mechanisms in acute response to trauma. Biological Psychiatry, 53, 779-788. doi: 10.1016/s0006-3223(02)01663-3

McNally, R. J., \& Robinaugh, D. J. (2011). Risk factors and posttraumatic stress disorder: Are they especially predictive following exposure to less severe stressors? Depression and Anxiety, 28, 1091-1096. doi: 10.1002/da.20867

Moore, S. A., Zoellner, L. A., \& Mollenholt, N. (2008). Are expressive suppression and cognitive reappraisal associated with stress-related symptoms? Behaviour Research and Therapy, 46, 993-1000. doi: 10.1016/j.brat.2008.05.001

Most, S. B., Chun, M. M., Widders, D. M., \& Zald, D. H. (2005). Attentional rubbernecking: Cognitive control and personality in emotion-induced blindness. Psychonomic Bulletin \& Review, 12, 654-661.

Nusslock, R., Shackman, A. J., Harmon-Jones, E., Alloy, L. B., Coan, J. A., \& Abramson, L. Y. (2011). Cognitive vulnerability and frontal brain asymmetry: Common predictors of first prospective depressive episode. Journal of Abnormal Psychology, 120, 497503. doi: $10.1037 / \mathrm{a} 0022940$

Ozer, E. J., Best, S. R., Lipsey, T. L., \& Weiss, D. S. (2003). Predictors of posttraumatic stress disorder and symptoms in adults: A meta-analysis. Psychological Bulletin, 129, 52-73. doi: 10.1037//0033-2909.129.1.52

Pfurtscheller, G., Stancak, A., \& Neuper, C. (1996). Event-related synchronization (ERS) in the alpha band - An electrophysiological correlate of cortical idling: A review. International Journal of Psychophysiology, 24, 39-46. doi: 10.1016/s01678760(96)00066-9

Pole, N., Neylan, T. C., Otte, C., Henn-Hasse, C., Metzler, T. J., \& Marmar, C. R. (2009). Prospective prediction of posttraumatic stress disorder symptoms using fear potentiated auditory startle responses. Biological Psychiatry, 65, 235-240. doi: 10.1016/j.biopsych.2008.07.015 
Rottenberg, J., Ray, R. R., \& Gross, J. J. (2007). Emotion elicitation using films. In J. A. Coan \& J. J. Allen (Eds.), The handbook of emotion elicitation and assessment (pp. 9-28). New York: Oxford University Press.

Rubin, D. C., Berntsen, D., \& Bohni, M. K. (2008). Memory-based model of posttraumatic stress disorder: Evaluating basic assumptions underlying the PTSD diagnosis. Psychological Review, 115, 985-1011. doi: 10.1037/a0013397

Schwarz, N. (2012). Why researchers should think "real-time": A cognitive rationale. In M. R. Mehl \& T. S. Conner (Eds.), Handbook of research methods for studying daily life (pp. 22-42). New York: Guilford Press.

Thibodeau, R., Jorgensen, R. S., \& Kim, S. (2006). Depression, anxiety, and resting frontal EEG asymmetry: A meta-analytic review. Journal of Abnormal Psychology, 115, 715729. doi: 10.1037/0021-843x.115.4.715

Thompson, R. (1991). Emotional regulation and emotional development. Educational Psychology Review, 3, 269-307.

Thrasher, S. M., Dalgleish, T., \& Yule, W. (1994). Information-processing in posttraumatic stress disorder. Behaviour Research and Therapy, 32, 247-254. doi: 10.1016/00057967(94)90119-8

Tomarken, A. J., Davidson, R. J., Wheeler, R. E., \& Doss, R. C. (1992). Individual differences in anterior brain asymmetry and fundamental dimensions of emotion. Journal of Personality and Social Psychology, 62, 676-687.

van Wingen, G. A., Geuze, E., Vermetten, E., \& Fernandez, G. (2011). Perceived threat predicts the neural sequelae of combat stress. Molecular Psychiatry, 16, 664-671.

van Zuiden, M., Geuze, E., Willemen, H. L. D. M., Vermetten, E., Maas, M., Amarouchi, K., . . Heijnen, C. J. (2012). Glucocorticoid receptor pathway components predict posttraumatic stress disorder symptom development: A prospective study. Biological Psychiatry, 71, 309-316. doi: 10.1016/j.biopsych.2011.10.026

Weidmann, A., Conradi, A., Grögera, K., Fehma, L., \& Fydrich, T. (2009). Using stressful films to analyze risk factors for PTSD in analogue experimental studies - Which film works best? Anxiety, Stress \& Coping, 22, 549-569.

Wessel, I., Merckelbach, H., \& Dekkers, T. (2002). Autobiographical memory specificity, intrusive memory, and general memory skills in Dutch-Indonesian survivors of the World War II Era. Journal of Traumatic Stress, 15, 227-234. doi: 10.1023/A:1015207428675 



\section{Chapter 2}

\section{Individual differences in spatial configuration learning predict the occurrence of intrusive memories}

Published as:

Meyer, T., Smeets, T., Giesbrecht, T., Quaedflieg, C. E. M., Girardelli, M., Mackay, G. N., \& Merckelbach, H. (2013). Individual differences in spatial configuration learning predict the occurrence of intrusive memories. Cognitive, Affective, \& Behavioral Neuroscience, 13, 186196. 


\section{Abstract}

The dual-representation model of posttraumatic stress disorder (PTSD; Brewin, Gregory, Lipton, \& Burgess, 2010, Psychological Review, 117, 210-232) argues that intrusions occur when people fail to construct context-based representations during adverse experiences. The present study tested a specific prediction flowing from this model. In particular, we investigated whether the efficiency of temporal-lobe-based spatial configuration learning would account for individual differences in intrusive experiences and physiological reactivity in the laboratory. Participants $(N=82)$ completed the contextual cuing paradigm, which assesses spatial configuration learning that is believed to depend on associative encoding in the parahippocampus. They were then shown a trauma film. Afterward, startle responses were quantified during presentation of trauma reminder pictures versus unrelated neutral and emotional pictures. PTSD symptoms were recorded in the week following participation. Better configuration learning performance was associated with fewer perceptual intrusions, $r$ $=-.33, p<.01$, but was unrelated to physiological responses to trauma reminder images $(p \mathrm{~s}>$ .46) and had no direct effect on intrusion-related distress and overall PTSD symptoms, $r s$ > -.12 , $p \mathrm{~s}>.29$. However, configuration learning performance tended to be associated with reduced physiological responses to unrelated negative images, $r=-.20, p=.07$. Thus, while spatial configuration learning appears to be unrelated to affective responding to trauma reminders, our overall findings support the idea that the context-based memory system helps to reduce intrusions.

\section{Keywords}

Posttraumatic stress disorder, intrusions, Spatial Contextual Cuing Task, startle paradigm 


\section{Introduction}

Many people are exposed to potentially traumatic events, such as life-threatening accidents, violent crimes, or the death of a close friend, at some point in their life. A possible negative outcome of the fear, horror, or helplessness that may accompany these events is posttraumatic stress disorder (PTSD). Trauma victims who develop PTSD suffer from prolonged reactions to the event, including re-experiencing (e.g., intrusions, nightmares), avoidance of cues and situations related to the trauma, emotional numbing, and increased general arousal (American Psychiatric Association, 2000). Surprisingly, only a minority of those who have been exposed to potentially traumatic experiences actually do develop PTSD. Indeed, most people are able to adapt well to adverse experiences, a phenomenon called resilience (Bonanno, 2004). Researchers and clinicians alike have been intrigued by the question of what distinguishes people who experience pathological symptoms after adversity from those who are resilient.

Studies of the predictive factors of PTSD strongly suggest that resilience is related to individual differences in bio-psychological reactions during and shortly after the traumatic event (e.g., Marmar et al., 2006; for a meta-analysis, see Ozer, Best, Lipsey, \& Weiss, 2003). For instance, alterations in physiological and hormonal activity known to influence memory have been shown to be predictive of later PTSD (Delahanty \& Nugent, 2006). Accordingly, a prominent theoretical account of PTSD (de Quervain, Aerni, Schelling, \& Roozendaal, 2009; Elzinga \& Bremner, 2002) posits that physiological hyperactivity and hormonal deregulation during a traumatic experience lead to dysfunctional activity in memory-encoding structures of the brain - notably, the amygdala and hippocampus - which could lead to the development of PTSD.

The dual-representation model by Brewin and colleagues (Brewin, Gregory, Lipton, \& Burgess, 2010) is a detailed neuroanatomical model of memory formation during traumatic experiences aiming to account for the above-mentioned findings (for competing or complementing models, see, e.g., Francati, Vermetten, \& Bremner, 2007; Rubin, Berntsen, \& Bohni, 2008; Shin, Rauch, \& Pitman, 2006; Suvak \& Barrett, 2011). According to this model, two distinct but interacting memory systems in the brain are responsible for the occurrence of intrusive memories, a hallmark symptom of PTSD. The first system is a sensation-based memory system, which would encode perceptual and affective qualities of events in cortical and subcortical sensory areas, including the insula and the amygdala. The processing of information within this system purportedly is enhanced during stressful situations. The second 
system is a context-based memory system that would support further processing of the sensory input both by recruiting sensory association areas and by translating the egocentric viewpoint of the sensory input into abstract, allocentric representations. The brain structures involved in context-based memory are the hippocampus and parahippocampus.

According to the dual-representation model (Brewin et al., 2010), intrusions occur when highly emotional sensation-based memories are formed in the absence of a complete contextbased memory for the traumatic event. When reactivated, a sensation-based memory will therefore be experienced from an egocentric viewpoint, such that the reactivated affective states are perceived as having immediate significance. Thus, according to Brewin et al., the formation of allocentric, context-based memory prevents or at least reduces the occurrence of intrusions. Therefore, one would expect variability in the efficiency with which contextual representations are encoded to underlie individual differences in resilience to adversity.

To date, the most direct evidence in support of the dual-representation model comes from two studies that assessed individual differences in visuospatial processing abilities and related them to PTSD symptoms (Bisby, King, Brewin, Burgess, \& Curran, 2010; Gilbertson et al., 2007). In an elegant twin study, Gilbertson et al. showed that PTSD was marked by impaired allocentric visuospatial processing, as measured with a 20 -item multiple-choice task in which participants had to rotate cubes in their minds or visualize how papers would be folded. More recently, Bisby et al. examined the occurrence of intrusions in a trauma film paradigm using a spatial memory task in which participants explicitly memorized spatial locations in a 3-D virtual space. Spatial learning was followed by recognition trials either from the same spatial viewpoint or from a shifted viewpoint, whereby the latter trials necessarily required a viewpoint-independent (i.e., allocentric) memory representation. Bisby and colleagues found that better recognition performance from a shifted viewpoint correlated with fewer perceptual intrusions. Thus, the aforementioned studies suggest that allocentric spatial processing and recognition may play an important role in intrusive memories. In line with the predictions of the dual-representation model (Brewin et al., 2010), these findings suggest that allocentric visuospatial processing and memory formation could be associated with individuals' ability to form contextualized memories of a traumatic experience.

The tasks used in both studies (Bisby et al., 2010; Gilbertson et al., 2007) required complex mental operations (e.g., explicit memorizing, mental orientation, visualization) that likely tapped the efficiency of the hippocampus (see Burgess, Maguire, \& O'Keefe, 2002), as well as structures in the parietal cortex (Jordan, Heinze, Lutz, Kanowski, \& Jancke, 2001). 
Importantly, the dual-representation model ascribes important roles to both the hippocampal area (e.g., the formation of context-based memories) and parietal regions in the development of intrusions (e.g., promoting the interaction between sensation-based and context-based memories; Brewin et al., 2010). Thus, the more specific involvement of a hippocampal-areabased memory system in the development of intrusions, as hypothesized in the dualrepresentation model, remains to be tested empirically.

To explore the specific link between hippocampal-area-based memory formation and intrusive experiences, we used the contextual cuing paradigm (Chun \& Jian, 1998) to assess individual differences in the efficiency with which visuospatial context information is encoded. This paradigm measures the degree to which spatial configurations of multiple simple cues are bound in memory. Although this learning performance is not necessarily allocentric in nature (e.g., Chua \& Chun, 2003), studies have suggested that contextual cuing crucially depends on structures in the parahippocampus (Chun \& Phelps, 1999; Manns \& Squire, 2001; Preston \& Gabrieli, 2008) that are involved in the encoding of associative feature conjunctions (Fyhn, Molden, Witter, Moser, \& Moser, 2004; Murray, Bussey, \& Saksida, 2007; van Strien, Cappaert, \& Witter, 2009) and serve as major input for the construction of spatial representations in the hippocampus (Fyhn, Hafting, Treves, Moser, \& Moser, 2007). Therefore, reduced learning efficiency on the contextual cuing task might reflect a reduction of information processing that would be a major input for a coherent hippocampal-dependent representation during a traumatic event. Thus, the contextual cuing paradigm can be used to provide additional insights into the relationship between contextual memory formation in the medial temporal lobe and intrusive memories of highly emotional events.

Aside from assessing the efficiency of visuospatial context-based memory formation, we subjected participants to a trauma film paradigm (Holmes, Brewin, \& Hennessy, 2004) and measured auditory startle responses $30 \mathrm{~min}$ later to assess affective responding (Jackson et al., 2003) to reminder pictures. Importantly, startle probes were presented both during and after picture presentation, making it possible to independently assess affective responding and affective down-regulation, respectively. Reminder-related affective responding may be especially relevant for individual differences in resilience, since PTSD patients, in contrast to resilient individuals, often show a specific exaggeration of startle responses toward traumarelated stimuli (Orr \& Roth, 2000). Heightened startle responses to fear-associated stimuli is indicative of amygdala activation (Davis, Walker, \& Lee, 1997), which is thought to be 
central in the processing of sensation-based memories (Brewin et al., 2010). Thus, in terms of the dual-representation model, the startle responses during memory activation may be indicative of the strength of film-related affective memories. By contrast, affective downregulation of startle responses is essentially thought to be mediated by the frontal cortex (Jackson et al., 2003) under guidance of contextual memory (Thayer, Ahs, Fredrikson, Sollers, \& Wager, 2012). Although the affective reaction to trauma memories may depend on a number of other mechanisms (e.g., selective attention, negative appraisals, avoidance, cognitive distortions; Brewin et al., 2010), the dual-representation model would predict that the efficiency of forming contextual representations of the trauma film contributes to affective down-regulation of startle responses. Finally, the occurrence of PTSD symptoms induced by the distressing film fragments was assessed using a 1-week intrusion diary and self-report measures of PTSD symptoms related to the trauma film.

On the basis of these considerations, we hypothesized that the efficiency of visuospatial context-based memory formation would be related to fewer intrusions in the week following exposure to the distressing film fragments. Additionally, we expected that this efficiency would lead to enhanced affect regulation in response to the activation of trauma memories as indexed by modulation of responses in the auditory startle paradigm. Concerning self-reported PTSD symptoms, more efficient context-based memory formation was expected to predict fewer PTSD symptoms in general (in terms of overall scores on PTSD scales) and fewer intrusions and lower intrusion-related distress in particular.

\section{Method}

\section{Participants}

Eighty-two undergraduates (63 women) with a mean age of 21.5 years $(S D=3.4)$ completed the study. Participants were recruited via advertisements at the university campus and received partial course credit or a small financial compensation in return for their participation. Exclusion criteria (based on self-report) were (1) recent psychological complaints, (2) drug or alcohol abuse or addiction, (3) blood phobia, and (4) past traumatic experiences that were similar to those depicted in the used trauma film fragments (see the Procedure section below; i.e., serious car accidents, life-threatening injuries, serious violence). Participants were informed that the materials used in the study might cause transient negative emotions and intrusions. In a follow-up inquiry, none of the participants reported long-term distress from the study. All participants had normal or corrected-to-normal 
vision. This study was approved by the standing ethical committee of the Faculty of Psychology and Neuroscience, Maastricht University. All participants provided written informed consent.

\section{Materials}

Spatial contextual cuing task. The contextual cuing paradigm (Chun \& Jian, 1998) requires participants to find a single target among a number of distractor stimuli. On half of the trials, the configuration of the distractor stimuli (i.e., the visuospatial context of the target) is repeated, while on the other half of the trials, new distractor configurations are presented. The repeated target contexts predict the location of the target and, thereby, facilitate the search, as evidenced by shorter reaction times (RTs) in comparison with new distractor configurations. This RT difference is an index of the contextual learning effect (Chun \& Jian, 1998). We used the abbreviated spatial contextual cueing task (SCCT) developed by Bennett and colleagues (Bennett, Barnes, Howard, \& Howard, 2009). Prior to the task, participants were given a passive strategy instruction in order to decrease performance variation due to different cognitive search strategies (see Lleras \& Von Muhlenen, 2004). Specifically, participants were asked to use their intuition rather than systematic search to find the target. The task consisted of 30 blocks containing six trials with repeated arrays and six trials with new arrays (for more details about stimuli and task construction, see Supplemental materials). RT data were condensed by averaging the median RTs across three epochs of 10 blocks. Similar to prior studies (e.g., Bennett et al., 2009), scores of configuration learning were calculated for each epoch by subtracting the score for repeated arrays from the score for novel arrays per epoch. Also, average accuracy scores were calculated for each epoch and array type.

We found the typical contextual cuing effect in the present sample (i.e., shorter RTs in repeated arrays, as compared with novel arrays; for details, Supplemental materials). We also observed strong non-sphericity in the array $\times$ epoch interaction, because participants with better initial configuration learning reached a ceiling of the learning effect already in the first epoch (see Figure 2.2 in Supplemental materials). Therefore, the average median RT difference in the first epoch $(M=96.4 \mathrm{~ms}, S D=411.4 \mathrm{~ms})$ most likely reflects individual learning differences and was used to index encoding efficiency (hereafter referred to as configuration learning performance). 
Eye-blink startle paradigm. In an eye-blink startle paradigm adapted from Jackson et al. (2003), participants viewed 30 reminder pictures from the distressing film fragments and 30 unrelated neutral (middle valence, low arousal ratings) pictures from the International Affective Picture System (IAPS; Lang, Bradley, \& Cuthbert, 2005). Additionally, 30 unrelated negative pictures (low valence, high arousal ratings) from the IAPS were included. This was done so as to contrast the expected modulation of startle responses to negatively valenced memories with a possible modulation of startle responses to acute negative affect induced by negative images ${ }^{1}$. The reminder pictures consisted of screen captures from the trauma film fragments, such that the used scenes contained no graphically disturbing details (i.e., the reminder pictures were chosen to be as neutral as possible by themselves). Using Self-AssessmentManikin (Bradley \& Lang, 1999) ratings, we confirmed in a small independent sample $(N=15)$ that the reminder pictures were more neutral than the negative pictures in terms of both valence $\left(M_{\text {Reminder }}=4.32 ; M_{\text {Negative }}=2.75\right), t(14)=-10.29, p<.001$, and arousal $\left(M_{\text {Reminder }}=5.23 ; M_{\text {Negative }}=6.3\right), t(14)=4.74, p<.001$. Reminder pictures had slightly lower ratings of valence than did neutral IAPS pictures $\left(M_{\text {Neutral }}=5.21\right), t(14)=$ $-8.16, p<.001$, as well as higher ratings of arousal $\left(M_{\text {Neutral }}=3.69\right), t(14)=5.69, p<.001$.

Pictures were shown for $6 \mathrm{~s}$ (interstimulus interval = $14 \mathrm{~s}$ ), accompanied by a $50-\mathrm{ms}$ white noise startle probe ( $95 \mathrm{~dB}$; near-instantaneous rise time) via headphones. Startle probe onset time was $2.5,4.5$, or $7 \mathrm{~s}$, relative to stimulus onset counterbalanced across stimulus categories, thus allowing the assessment of the chronometry of responding for each of the stimulus categories. In particular, the 2.5- and 4.5-s probes served to quantify affective responding, whereas the 7-s probe served to assess the degree of affective down-regulation (Jackson et al., 2003). The trial sequence was randomized individually for each participant, with the restriction that no more than three consecutive trials had the same probe timing or picture category. Six additional trials without startle probe were inserted into the trial sequence (two per picture category) in order to reduce the predictability of the startle probes.

1 IAPS pictures used for the neutral control category were 2190, 2200, 2215, 2397, 2441, 2745.1, 5120, 5500, 5532, 5740, 7000, 7004, 7010, 7020, 7025, 7031, 7050, 7060, 7080, $7090,7100,7130,7150,7160,7170,7175,8180,7224,7233,7500,7550,7700$; IAPS pictures used for the negative control category were 1052, 1070, 1090, 1120, 1220, 1280, 1300, 2120, 2691, 3000, 3010, 3016, 3071, 3100, 3130, 3150, 3225, 3261, 3500, 3530, 6020, 6190, 6200, 6230, 6313, 6370, 6510, 9040, 9254, 9410, 9423, 9490. 
Following the guidelines of Blumenthal et al. (2005), electromyography (EMG) was sampled continuously at $1000 \mathrm{~Hz}$, using $\mathrm{Ag} / \mathrm{AgCl}$ electrodes below the participants' left eye and an electrode on the forehead serving as signal ground. Electrode impedances were kept below $5 \mathrm{kOhms}$. EMG signals were rectified and $30 \mathrm{~Hz}$ low-pass filtered. For each trial, EMG signals were extracted from -50 to $250 \mathrm{~ms}$ relative to probe onset. The 50 -ms period before probe onset was used for baseline correction after rejection of noise-contaminated trials (i.e., signal changes $>20 \mu \mathrm{V}$ during baseline or reflex onset before probe onset). Data from 1 participant had to be excluded due to excessive artefacts. Reflex onset and peak values were extracted automatically between 20 and $120 \mathrm{~ms}$ after probe onset, yielding a startle magnitude per trial (peak minus onset values). Trials without eyeblink response were rated as zero. Startle magnitudes were square root transformed and averaged within subjects for each stimulus category and startle probe time. Startle potentiation (i.e., the relative strength of startle magnitude during reminder and unrelated negative trials, as compared with unrelated neutral trials) was statistically significant at the $4.5-\mathrm{s}$ probe timing (for details, see Supplemental materials). For further analyses, startle potentiation scores were computed for reminder and unrelated negative trials per probe timing.

Assessment of PTSD symptoms. Intrusions were assessed using a 1-week diary (Holmes, James, Coode-Bate, \& Deeprose, 2009), with instructions to record intrusions as soon as they occurred. If no intrusions had occurred, participants were to record the absence of intrusions at least twice a day. The diary required participants to briefly describe each intrusion (for verification) and to indicate whether the intrusion was predominantly based on images, thoughts, or both. Intrusion frequencies were log-transformed prior to analyses to correct their right-skewed distribution. For each intrusion, participants also indicated how distressing it was on an 11-point scale (anchors: $0=$ not at all; $10=$ extremely). Distress scores were averaged across all recorded intrusions. Intrusion-related distress was assessed additionally using the Intrusion Symptoms subscale of the Impact of Events Scale (IES; Horowitz, Wilner, \& Alvares, 1979) $(\alpha=.76)$ and the Re-Experiencing subscale of the selfreport PTSD Symptom Scale (PSS-SR; Foa, Riggs, Dancu, \& Rothbaum, 1993) ( $\alpha=.57$ ). The total scores of both questionnaires were used to assess overall PTSD symptoms $(\alpha=.83$ and .76 , respectively). Both questionnaires were adapted to measure symptoms related to the trauma film.

Mood, anxiety, and depression. The Positive and Negative Affect Schedule (PANAS; Watson, Clark, \& Tellegen, 1988) was used for repeated measurements of current positive 
affect (PA) and negative affect (NA) ( $\alpha$ s $>$.74). The trait subscale of Spielberger's State-Trait Anxiety Inventory (STAI-T, Dutch translation; Van der Ploeg, 1985) was used to control for stable individual differences in anxiety levels $(\alpha=.89)$. Likewise, a Dutch version of the Beck Depression Inventory (Bouman, Luteijn, Albersnagel, \& Van der Ploeg, 1985) was used to control for general levels of depression $(\alpha=.87)$.

\section{Procedure}

Participants were invited to two laboratory sessions separated by a 1-week interval in which a diary was completed. During the first session, participants were seated in front of a computer screen at approximately $56 \mathrm{~cm}$ unrestrained viewing distance and completed a battery of computer-administered baseline questionnaires (not all questionnaire data are presented here), followed by the contextual cuing paradigm. Next and after preparing the skin with abrasive gel, the electrodes for the startle paradigm were attached. After that, the PANAS was administered, followed by a 14-min presentation of emotional video fragments. The stimulus materials largely overlapped with those used in Holmes et al. (2009), extended with similar video fragments. Participants were instructed to imagine being a witness to the scenes that consisted of explicit footage of genocide, medical surgeries, a drowning scene, and a car accident. During the trauma film presentation, participants were monitored via a closed-circuit video system to ensure adherence to the instructions ${ }^{2}$. After the trauma film presentation, mood was reassessed with the PANAS. Following an unrelated and simple filler task (the Attention Network Test; Fan, McCandliss, Sommer, Raz, \& Posner, 2002) lasting 30 min, participants underwent the eye-blink startle paradigm. At the end of the session, the electrodes were removed, and an appointment for a second session 1 week later was scheduled (with a tolerated deviation of 1 day). Finally, participants were given the diary and returned after 1 week to complete the self-report questionnaire of PTSD symptoms.

\section{Statistical analysis}

To test our hypotheses, we employed Pearson product-moment correlations and multiple regressions, testing linear associations between independent and dependent variables. Repeated measures ANOVAs and $t$-tests were also used. When sphericity assumptions for

2 Two participants looked away for short periods of time during the film presentation but were not excluded from the sample. 
ANOVAs were violated, Greenhouse-Geisser corrected statistics are reported. ANOVAs are supplemented with partial eta squared $(\eta p 2)$ and $t$-test with Cohen's $d$ as effect size estimates. Alpha was set at .05 (two-tailed) for all analyses.

\section{Results}

\section{Effects of the trauma film}

Affect changes due to the trauma film were assessed by comparing pre- and post-film PANAS PA and NA scores using paired-samples $t$-tests. PANAS data from 1 participant were lost due to a database error in the electronic questionnaire system. Overall, PA decreased $\left(M_{\text {difference }}=-2.15, S D=4.37\right), t(80)=-4.4, p<.001, d=-.39$, whereas NA increased $\left(M_{\text {difference }}=3.88, S D=4.60\right), t(80)=7.6, p<.001, d=.73$, following the trauma film. The mean scores of PTSD symptoms are summarized in Table 2.1.

\section{Configuration learning performance (SCCT) and startle responses}

Linear associations between SCCT learning and startle potentiation scores were tested independently for each type (reminder, unrelated negative) and probe timing $(2.5,4.5,7 \mathrm{~s})$. SCCT learning was unrelated to the two types of startle potentiation scores at the timings 2.5 and $4.5 \mathrm{~s}$ ( $r$ s ranging from -.08 to $.02, p \mathrm{~s}>.46$ ). Neither was there an association with potentiation scores at the 7 -s timing for reminder trials, $r=-.06$, n.s., whereas the correlation for negative trials approached significance, $r=-.202, p=.070$. In order to take all three timings and both types of startle potentiation into account, SCCT learning was entered as a two-level between-subjects factor (group allocation by median-split) in a 2 (group) $\times 2$ (type) $\times 3$ (timing) repeated measures ANOVA. No significant interaction of group by timing or by type $(p s>.68)$ was found, although the three-way interaction was borderline significant, $F(2,158)=2.66, p=.07, \eta p 2=.03$. Despite the absence of statistical significance, we explored this interaction with post-hoc paired-samples t-tests, which suggested that the group with poor learning on the SCCT $(n=40)$ showed larger startle potentiation during negative trials, as compared with reminder trials, at the 7-s timing, $t(39)=2.18, p=.035, d=.29$, whereas no such difference existed in the group with good SCCT learning $(n=41), t(40)=$ $-0.32, p=.75, d=-.07$. 


\section{Configuration learning performance and PTSD symptoms}

Direct linear associations between configuration learning performance and PTSD symptoms (i.e., intrusion frequency, distress, and overall symptoms) were assessed using correlation analyses. The results are summarized in Table 2.1. A significant negative correlation between SCCT learning and the total number of reported intrusions was found. This relationship was carried by intrusions that were perceptual (i.e., mental images), whereas SCCT learning was unrelated to the number of intrusions with a verbal component (i.e., thoughts or thought-image combinations). On the other hand, intrusion-related distress was unrelated to SCCT learning irrespective of assessment method, as were the total symptom scores on the PTSD scales.

Since one may hypothesize that other factors might be responsible for the effect of SCCT learning on the frequency of image-based intrusions, we tested this relationship while statistically correcting for trait anxiety, depression, and affective responding to the trauma film (i.e., PA and NA change scores). We therefore included the latter four factors in a multiple linear regression model (stepwise method; removal criterion: $p>.10$ ) and entered the factor SCCT learning subsequently. Only increases in NA due to the trauma film were kept as a significant predictor of perceptual intrusions, and entering SCCT learning significantly enhanced the model $\left(R_{\text {change }}^{2}=.10\right), F(1,78)=9.61, p<.01$. In this model, higher SCCT learning again predicted fewer image-based intrusions, $\beta=-.32, p<.01$, whereas stronger NA change predicted more image-based intrusions, $\beta=.27, p<.01$. 


\begin{tabular}{llcc}
\hline PTSD Symptoms & & $\begin{array}{c}\text { Sample Mean } \\
(S D)\end{array}$ & $\begin{array}{c}\text { Correlation (r) Configuration } \\
\text { Learning Performance }\end{array}$ \\
\hline Intrusion Frequency & & \\
Method & Scale & $2.76(3.16)$ & $-.33^{* *}$ \\
Intrusion Diary ${ }^{\text {a }}$ & Images & $0.39(0.83)$ & .09 \\
& Thoughts & $1.06(1.77)$ & -.06 \\
& Images/Thoughts & $4.21(4.15)$ & $-.28^{* *}$ \\
Intrusion Distress & Sum & & -.12 \\
Method & Scale & $3.50(2.04)$ & -.05 \\
Intrusion Diary & Distress & $6.02(5.24)$ & -.02 \\
IES & Intrusions & $3.34(1.81)$ & \\
PSS-SR & Re-experiencing & & -.03 \\
Overall PTSD Symptoms & Scale & & \\
Method & Total Score & $9.95(8.56)$ & \\
IES & Total Score & $6.30(4.69)$ & \\
PSS-SR & & & \\
\hline
\end{tabular}

Table 2.1. Pearson product-moment correlations between configuration learning performance and PTSD symptoms $(N=82)$. Configuration learning performance was calculated as the average median difference in reaction time between novel and repeated arrays across the first epoch of the Spatial Contextual Cueing Task. IES = Impact of Event Scale; PSS-SR = PTSD Symptom Scale - Self Report. ${ }^{a}$ The numbers of intrusions were log transformed prior to calculating correlation coefficients, i.e., $\ln (1+\#$ intrusions $)$.

$* * p<.01$ (two-tailed). 


\section{Discussion}

The present study explored whether efficiency in learning visuospatial context information might be a relevant factor to explain individual differences in resilience after potentially traumatic experiences. The main findings of our study can be summarized as follows. We found that better configuration learning performance in a contextual cuing paradigm predicted significantly fewer intrusions during a 1-week period - specifically those intrusions that were purely perceptual in nature - over and above affective responding to the trauma film. In contrast, configuration learning performance was not predictive of intrusionrelated distress and overall PTSD symptoms. Neither was there a direct relationship between learning performance in the contextual cuing paradigm and startle potentiation during or shortly after presentation of reminder pictures, aiming to activate memories of the trauma film. However, configuration learning performance tended to be negatively related to startle potentiation scores after the offset of unrelated negative pictures. Moreover, only in participants with relatively poor configuration learning performance, the latter potentiation scores appeared to be higher, as compared with potentiation scores of reminder pictures.

\section{Efficiency of configuration learning specifically reduces perceptual intrusions}

Individuals with better configuration learning performance showed more resilience in terms of lowered levels of intrusions. This effect was specific for perceptual intrusions, possibly because they are generated differently than verbal intrusions. For instance, perceptual intrusions differ with respect to their level of abstraction and impact on emotion (Holmes \& Mathews, 2010), and it has been shown that the occurrence of intrusive thoughts can be manipulated independently of the occurrence of image-based intrusions (Hagenaars, Brewin, van Minnen, Holmes, \& Hoogduin, 2010). Our study replicates and extends the findings of Bisby et al. (2010), who demonstrated a negative correlation between allocentric spatial recognition performance and perceptual intrusions in a trauma film paradigm. Differences between the contextual cuing paradigm employed in the present study and the recognition task used by Bisby and colleagues may be of relevance for theories of intrusions. They instructed participants to explicitly memorize spatial locations in a 3-D virtual space and to recognize configurations from a shifted viewpoint. This task requires a number of complex operations, including visualization, mental rotation, and explicit recognition, each of which may be (differently) related to the occurrence of intrusions. In contrast, in the contextual cuing paradigm, participants rely on repeated visual context information to aid search performance 
independently of, or even in the absence of, explicit recognition (Chun \& Phelps, 1999). Also, the contextual cuing effect may depend on a viewpoint-dependent rather than an allocentric spatial representation in memory (e.g., Chua \& Chun, 2003). Therefore, our data show that also less abstract and less complex forms of visuospatial scene processing may be relevant in the prevention of perceptual intrusive experiences.

More speculatively, our findings can also be reconciled with the neuroanatomical predictions of the dual-representation model (Brewin et al., 2010), in which the hippocampus and parahippocampus play an essential role in the creation of coherent contextual memories and in the reduction of intrusive memories. The contextual cuing paradigm measures spatial memories that are processed implicitly in the parahippocampus (Preston \& Gabrieli, 2008), and more efficient spatial learning on this task would be expected to increase the input of spatial representations from the parahippocampus to the hippocampus (e.g., Fyhn et al., 2007). Therefore, our finding that higher learning efficiency in the contextual cuing paradigm is associated with fewer intrusions is in line with the view that memory formation in the hippocampal area helps to prevent intrusions, possibly by enabling the individual to form a coherent contextualized representation of a traumatic event.

Importantly, the present study failed to observe more global effects of contextual learning efficiency on clinically relevant outcomes such as distress and overall PTSD symptoms. This seems to contradict the previous findings by Gilbertson et al. (2007) showing a negative association between spatial configuration processing and overall symptom severity in PTSD patients. A number of reasons might account for the different findings. First, Gilbertson et al. assessed symptoms in PTSD patients, whereas we experimentally induced symptoms in individuals without prior psychopathology. It is therefore possible that visuospatial processing performance plays different roles in ongoing symptomatology and in the initial encoding process after viewing a distressing film. Second, and similar to the study of Bisby et al. (2010), the task used by Gilbertson et al. involves complex operations such as mental orientation and visualization, and these processes might be differently involved in the development and maintenance of PTSD symptoms than is learning performance in the contextual cuing paradigm. For instance, one might speculate that visualization requires efficient processing in parietal areas (Jordan et al., 2001), which have been proposed to play an important role in the interaction between sensation-based and context-based memories in the dual-representation model (Brewin et al., 2010). Finally, Gilbertson et al. noted that their task was cognitively demanding, which introduces the possibility that their results may have 
been confounded by intelligence. Configuration learning in the SCCT occurs implicitly (i.e., with little cognitive demand) and can be readily distinguished from procedural learning (i.e., overall performance; Preston \& Gabrieli, 2008). At the same time, our sample consisted of relatively high-functioning individuals. Together, these two factors likely reduce potentially confounding effects of intelligence on task performance and symptom development. In sum, our and Gilbertson et al.'s findings suggest that visuospatial contextual encoding efficiency, likely reflecting activation in the parahippocampus, plays a crucial but specific role in the development of perceptual intrusions, whereas other mechanisms probably determine individual differences in the distress caused by intrusions (e.g., negative appraisals once an intrusion has occurred).

\section{Efficiency of configuration learning and startle responses}

In order to explore whether configuration learning performance would be related to affective responding to trauma reminders, we used a startle paradigm, in which trauma film reminder pictures, as well as unrelated negative and neutral control pictures, were presented. We found no indication that configuration learning performance was linked to startle responses during or shortly after the presentation of reminder pictures. Also, we were unable to find a relationship between learning performance and the course of startle responding across the three startle timings, meaning that we failed to demonstrate the expected effect on the chronometry of affective responding to trauma film reminders. Thus, we found no support for the hypothesis that better configuration learning performance would lead to enhanced affective down-regulation (i.e., startle responding at the 7-s timing). This hypothesis was based on the assumption that SCCT performance is related to enhanced context-based memory formation, which would, in turn, be beneficial in the affect regulation controlled by the frontal cortex (Thayer et al., 2012).

One possible implication of our results is that affective processing of trauma-filmrelated memories may not be influenced by individual differences in the efficiency with which visuospatial context information is encoded. In terms of the dual-representation model (Brewin et al., 2010), our findings could suggest that the parahippocampal and amygdalabound components in this model are not necessarily coupled. More generally, it is also possible that contextual embedding of a traumatic experience in memory does not necessarily imply that the affective response to the trauma cue will be dampened. Thus, other processes that have been suggested to play a role in the affective reaction to trauma memories may 
prove to be more important, including selective attention, negative appraisals, avoidance, and cognitive distortions (Brewin et al., 2010). However, since SCCT performance does not reflect contextual embedding of trauma memories directly, our data do not imply the absence of this link. Interestingly, the startle responses after unrelated negative pictures showed a trend toward affective hyper-responsivity in individuals with poorer contextual learning performance. This finding was unexpected, since configuration learning performance was expected to correlate with enhanced affect regulation only in response to affect-laden memories, but not during acute induction of negative affect. Although this trend-significant association was small and thus requires replication, one might speculate that this pattern of results indicates that the context-based memory system promotes contextual embedding when one is currently confronted with unrelated aversive stimuli, but not when reactivated trauma memories are processed.

Notably, the pattern of affective responding in our paradigm differed slightly from that in an earlier study with a similar method. That is, in Jackson et al. (2003), 47 participants displayed potentiated startle responses to negative images, irrespective of the timing at which the probe was presented. In contrast, we found that statistically significant startle potentiation during reminder and unrelated negative images occurred only at the $4.5 \mathrm{~s}$-timing, but not at 2.5 and $7 \mathrm{~s}$. Although it is unclear why these findings differ from previous ones, they are entirely in line with the expectation that affective responding would be strongest at $4.5 \mathrm{~s}$ (i.e., when the content of the picture has been fully apprehended), and weaker at $2.5 \mathrm{~s}$ (i.e., during initial visual processing) and $7 \mathrm{~s}$ (i.e., $1 \mathrm{~s}$ after picture offset, when emotion regulation occurs). Thus, both the reminder pictures and negative unrelated pictures evoked a heightened affective reaction, which was down-regulated after picture offset. Importantly, the startle potentiation at $4.5 \mathrm{~s}$ did not differ between the trials with reminder pictures and unrelated negative pictures, although the graphical content was more aversive in the latter pictures. This indicates that startle potentiation in the reminder pictures is likely caused by the activation of affect-laden memories. The subsequent affective down-regulation appeared to be stronger for reminder pictures, since the startle magnitudes at $7 \mathrm{~s}$ were even lower than for neutral images, which was not the case for unrelated negative pictures. Thus, the pattern of findings suggests that we were able to adequately capture the time course of affective responding to trauma reminders, in order to investigate the relationship to individual differences in spatial configuration learning. 


\section{Assessing individual differences with the contextual cuing paradigm}

To our knowledge, the present study is the first to use the contextual cuing paradigm (Chun \& Jian, 1998) in the context of individual-differences research. Using the SCCT (Bennett et al., 2009), we observed the typical contextual cuing effect, defined as reaction time advantage during visual search in repeated versus novel spatial configurations. Variation (i.e., individual differences) in this learning performance was strongest at the beginning of the task. In fact, individuals with low initial learning performance significantly improved in the course of the task, whereas individuals with high initial learning performance did not. Thus, even though individuals may differ little in learning performance in the final epoch of the task, there is considerable variation in the speed at which the learning effect is acquired. In other words, individual differences in the efficiency with which contextual information was learned apparently manifested themselves early rather than late during the task. We therefore decided to base our estimate of configuration learning performance on individual differences during the first ten repetitions of configurations (i.e., the first epoch). Moreover, learning performance was negatively associated with engagement in an active search strategy in the second epoch, but only in individuals with high initial learning performance. In line with this approach, Preston and Gabrieli (2008) showed that learning-related activation in the parahippocampus is strongest at the beginning of the task and declines after as few as two repetitions. This implies that in order to measure contextual learning efficiency, it may not be necessary to present a large number of visuospatial context repetitions.

\section{Limitations}

The following limitations deserve to be mentioned. To begin with, the present study was based on the assumption that spatial configuration learning would be related to the formation of context-based memories as hypothesized in the dual-representation model (Brewin et al., 2010). Although SCCT performance can be linked theoretically to the efficiency of the context-based memory system, we cannot exclude the possibility that the negative association between SCCT performance and intrusions was mediated by psychological processes other than the formation of context-based memories. That is, the present data cannot answer the question whether individuals who are efficient in spatial configuration learning applied this efficiency to bind the features of the trauma film fragments. Therefore, future studies using a more direct assessment of contextualized memories next to configuration learning performance are needed. Also, the present study measured configuration learning 
performance only in relation to PTSD symptoms and involuntary memory (i.e., intrusions), but we did not assess explicit and voluntary memory performance. Therefore, no conclusions can be drawn about the specificity of SCCT effects on involuntary memory.

Another limitation is that the trauma film paradigm was used as an analogue condition to a traumatic event. It may be argued that trauma films are unable to elicit responses comparable to those of a potentially traumatic experience in real life. Also, we inserted a filler task (the Attention Network Test; Fan et al., 2002) to standardize the time between the trauma film and the subsequent startle paradigm. Although this task is very simple, it requires visuospatial attention, which may have had a dampening effect on the occurrence of intrusions (e.g., Holmes et al., 2009). Thus, a weak effect of the trauma film and, consequently, reduced statistical power may provide an alternative explanation for the absence of associations between configuration learning performance and the clinical and physiological parameters.

Another potential limitation is related to the use of reminder pictures to activate trauma memories in the startle paradigm. Since these images were selected to depict the low distressing aspects of the trauma film fragments, it is possible that full activation of the trauma film memories required a conscious retrieval process. Hypothetically, individuals with lower configuration learning performance in the SCCT may also have been marked by less efficient retrieval of the trauma film memories, which would have cancelled out possible effects of configuration learning performance on startle responding. Although we cannot rule out this possibility, we found nevertheless that startle responses during full appraisal of neutral reminder pictures were significantly potentiated, as compared with unrelated neutral pictures, this potentiation being just as strong as that for unrelated negative pictures (see Supplemental materials). This suggests that the startle paradigm readily activated traumafilm-related memories and provided valid measurements of affective responding.

Finally, a potential threat to the external validity is related to our sample (i.e., undergraduates), which might be more skilled than the general population in emotion regulation and the adjustment to emotional provocation. Also, although we excluded participants who indicated high self-relevance of the trauma film (i.e., a history of specific traumatic experiences) and previous psychiatric problems on a self-report screening form, we did not systematically assess pre-existing PTSD symptoms, which may have influenced the results of this study. 


\section{Future considerations and conclusion}

The present results have several implications for future research in the area of resilience and PTSD. First, while the present study demonstrated that more efficient spatial configuration learning contributes to individual differences in resilience in that it is related to lowered intrusion levels, the mechanism that mediates this effect remains obscure. Future studies may want to explore more directly the link between spatial configuration learning and the formation of context-based memories. For instance, the dual-representation model (Brewin et al., 2010) suggests that abstracted encoding of environmental patterns eventually helps to respond flexibly in novel situations and enhances higher cognitive functions, including narration and communication. Thus, one possible pathway from spatial configuration learning to the development of intrusions might be mediated by facilitated building of contextual memories, which would, in turn, lead to a better understanding of the stressful experience. However, it is also possible that the efficiency of spatial configuration learning is a mere epiphenomenon of intrusions, because individuals with more efficient parahippocampal-area-based configuration learning may also use the same brain area more efficiently for an unrelated basic psychological process, which may lead to suppression of intrusions. These issues remain to be addressed by future research.

Second, more efficient spatial configuration learning did not appear to inhibit affective processing of trauma reminders in terms of physiological responding, raising the question of whether the (parahippocampal component of the) context-based memory system contributes directly to affect regulation in reactivated trauma memories. Another interesting aspect is that there were (relatively weak) indications that individuals with more efficient configuration learning regulated their responses to unrelated negative pictures more efficiently. On the basis of this pattern of findings, one could speculate that more efficient spatial configuration learning indeed has a beneficial impact on the time course of affective responding, but only during the initial encoding of aversive perceptual information. Future studies might want to clarify this issue, next to investigating whether and how spatial configuration learning and context-based memory embedding contribute to resilience at later stages after a trauma-for instance, when trauma memories are retrieved and reconsolidated.

Third, both similarity and disparity of the present findings with previous studies into the role of spatial processing in PTSD (Bisby et al., 2010; Gilbertson et al., 2007) should inspire future studies to address important details of the theorized context-based memory system (Brewin et al., 2010). For instance, while the specific effects on intrusion frequency reported 
here can be plausibly linked to implicit learning in the parahippocampus (Preston \& Gabrieli, 2008), it remains to be seen how such implicit learning processes might interact with more complex and explicit spatial processing (e.g., in the hippocampus) in the development of PTSD symptoms.

Finally, the finding that spatial configuration learning efficiency predicted perceptual intrusions, but not intrusion-related distress, may suggest that a combination of several factors accounts for the development of PTSD, each factor making rather specific contributions. Therefore, one could speculate that an inefficient processing in parts of the context-based memory system represents a nonspecific risk factor that generally increases the likelihood of experiencing more sudden recollections (not necessarily aversive ones; see Rubin, Boals, \& Berntsen, 2008). The actual development of PTSD after aversive experiences might thus depend additionally on other factors, such as the idiosyncratic interpretation of these intrusions and the cognitive-behavioural reaction to them (e.g., Steil \& Ehlers, 2000).

In conclusion, the present study demonstrates that the theoretical framework of the dualrepresentation model (Brewin et al., 2010) can be helpful in identifying and investigating a number of core mechanisms of resilience. Our results lend partial support to this model and suggest that efficiency in forming visuospatial context memories plays a specific role in image-based intrusive memories. Meanwhile, the distress related to intrusions and other PTSD symptoms appear to be unrelated to spatial configuration learning, prompting future research to investigate the role of context-based memory formation in memory-related affect regulation. Thus, in order to understand resilience, it might be helpful to study the memory systems proposed by the dual-representation model in interaction with other factors such as cognitive-behavioural reactions to the occurrence of intrusions.

\section{Supplemental Materials}

\section{Construction of the contextual cueing paradigm}

Stimuli. Trials consisted of grey screen displays in which a white ' $\mathrm{T}$ '-shaped target symbol, rotated randomly either 90 or 270 degrees, was presented among eleven white 'L'shaped distracters, each rotated $0,90,180$, or 270 degrees. The leg of the ' $L$ '-shaped distracters was offset by 3 pixels for higher similarity with the target item. All twelve items were placed on an invisible 6 rows x 8 columns grid, and then repositioned with $+/-3$ pixels along each axis to avoid colinearity. Each of the 12 items occupied about 1.1 degrees of visual angle at $56 \mathrm{~cm}$ viewing distance. The target item could not occur in the four corner cells and 
not in the four centre cells of the grid, and the target location (i.e., distance from center and sides of the screen) was balanced across trials. Each trial started with a fixation cross for 1 sec, followed by a configuration display that required participants to indicate as quickly and accurately as possible whether the base of the target stimulus pointed left or right by pressing response keys ('z' or '/') on a keyboard. The configuration display was presented for $10 \mathrm{sec}$ or until the participant responded. Auditory feedback was provided upon response (a highpitch or low-pitch tone for correct or incorrect / too slow responses, respectively), before the next trial started. Figure 2.1 displays an example display.

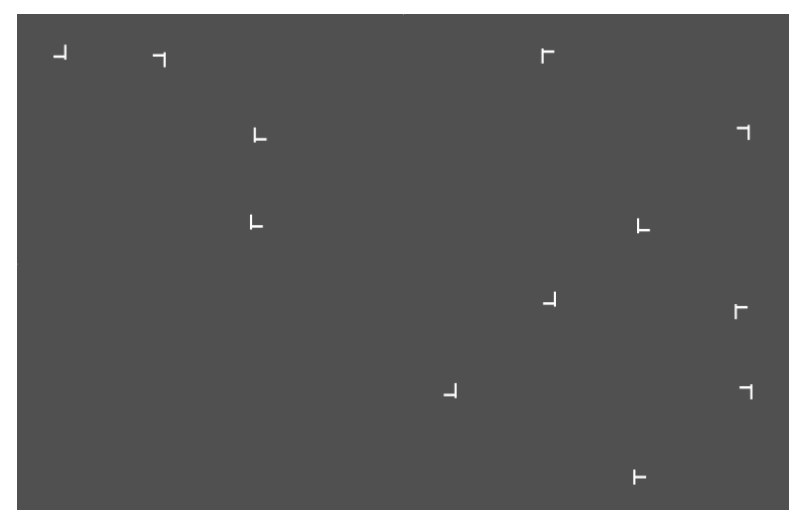

Figure 2.1. A typical visual search display in the Spatial Contextual Cueing Task. Participants were required to indicate the orientation of the ' $\mathrm{T}$ ' shaped target symbol.

Task procedure. The task consisted of 30 blocks with 12 trials each. Six trials in each block were repeated arrays, whereas 6 trials were new arrays. In repeated arrays, both target positions and distracter configuration remained exactly the same across blocks. By contrast, each of the six new arrays had a fixed target position (not overlapping with target positions of the repeated arrays) that remained constant across blocks, but the distracter configuration was newly randomized in each block. The target direction (' $\mathrm{T}$ ' pointing left or right) was completely randomized across all trials. Thus, the probability to find a target at a specific location (1 in 12) and pointing in a specific direction (1 in 2) was equal in all trials, only repeated arrays providing additional information about the target location through the repetition of the distracter configuration. The order of trials with repeated vs. new arrays was randomized within each block. In addition to the 6 repeated and 180 new arrays that were used, 12 new arrays were created for practice trials prior to the first block of the task. After task completion, participants were asked whether they had noticed the repetition of some 
arrays, and if so, whether repetitions subjectively facilitated the search. This was done to assess whether conscious awareness had an influence on learning performance.

\section{Contextual cueing effect in the SCCT}

To test whether the SCCT showed the typical contextual cueing effect in the whole sample, a 2 (array: novel, repeated) by 3 (3 epochs) repeated measures ANOVA was performed both for RT and for accuracy scores. For RT, a significant main effect of epoch was found, Greenhouse-Geisser corrected $F(1.7,134.3)=163.2, p<.001, \eta 2 p=.67$, showing shorter RTs as a function of practice. Spatial configuration learning was shown by a significant main effect of array, $F(1,81)=38.7, p<.001, \eta 2 p=.32$ and by a significant array by epoch interaction, Greenhouse-Geisser corrected $F(1.7,138.0)=7.9, p=.001, \eta 2 p=.1$, indicating that RT was shorter in repeated arrays than in novel arrays and that this difference increased with practice. Strong non-sphericity in the array by epoch interaction (Mauchly's W $=.82$, Chi-Square $=15.3, p<.001)$ suggested that individual differences in spatial configuration learning were strongest in the first epoch and decreased with practice. In order to assess whether some participants might have reached a ceiling of configuration learning already in the first epoch, we performed a repeated measures ANOVA of learning score across the three epochs with learning score in the first epoch as between-subjects factor (median-split: superior vs. inferior learning), revealing an epoch by group interaction, $F$ ( 2 , $160)=26, p<.001, \eta 2 p=.25$. That is, configuration learning across epochs occurred only in the lower learning group $(n=40), F(2,78)=38.6, p<.001, \eta 2 p=.50$, whereas a ceiling effect in the higher learning group $(n=42)$ was evidenced, $F(2,82)=1.7, p=.186, \eta 2 p=$ .04. Therefore, the average median RT difference in the first epoch was subsequently used to index the efficiency of context-based memory formation (hereafter referred to as contextual learning performance). Contextual learning performance did not differ between those unaware of the array repetitions $(n=40)$, those who noticed the repetitions and reported subjective facilitation $(n=30)$, and those who noticed but reported no subjective facilitation $(n=12), F$ $(2,79)=0.11, n s, \eta 2 p<.01$. Overall accuracy was close to ceiling $(M=96.7 \%, S D=.04)$, which probably accounts for the absence of main or interaction effects of array on accuracy $(p s>.18, \eta 2 p<.02)$. 


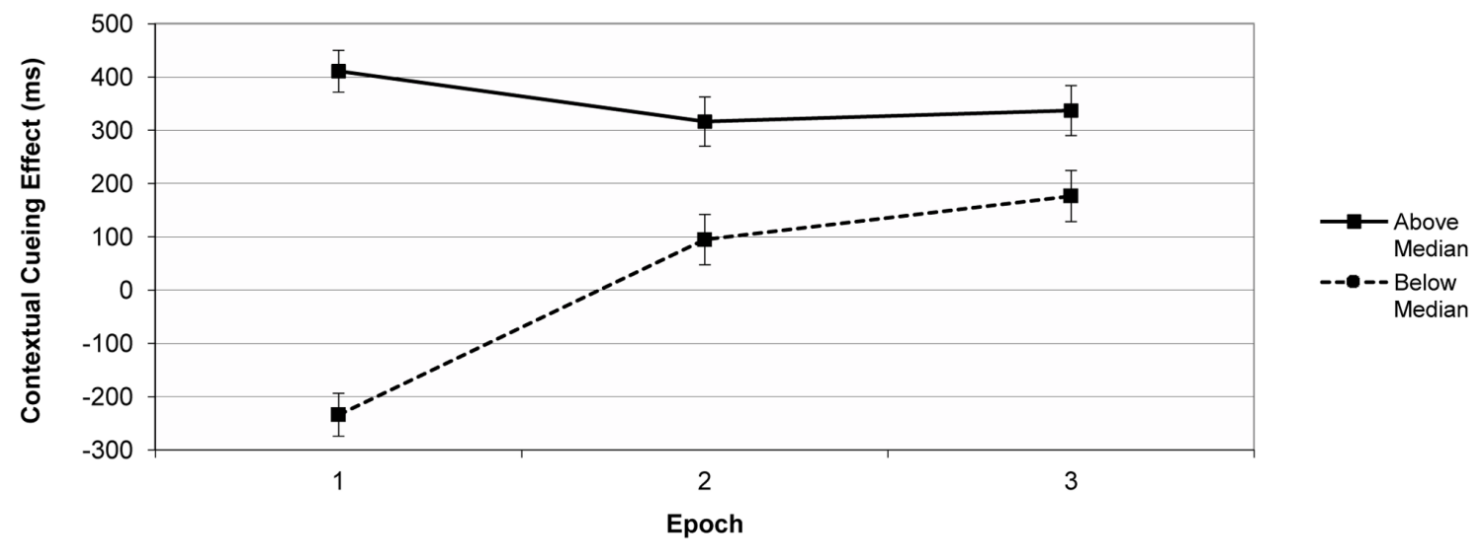

Figure 2.2. Contextual cueing effect (RT-novel minus RT-repeated) across epochs separately for superior vs. inferior learners in the first epoch. Participants with above-median contextual cueing effect in the first epoch $(n=42)$ reached a ceiling of the learning effect, whereas participants with below-median performance $(n=40)$ still improved across epochs. Therefore, the contextual cueing effect in epoch 1 reflects most individual differences in contextual learning efficiency. Error bars indicate standard errors of measurement.

\section{Startle response effects}

To assess startle potentiation (i.e., the relative strength of startle magnitude during negative and reminder pictures as compared to neutral pictures), a 3 (startle timing: $2.5 \mathrm{~s}, 4.5 \mathrm{~s}, 7 \mathrm{~s}$ ) by 3 (stimulus category: reminder, negative, neutral) repeated measures ANOVA was performed, showing a significant category by timing interaction, $F(4,320)=3.62, p=.007, \eta 2 p=.04$. Post-hoc paired-sample $t$-tests showed that relative to neutral trials, startle magnitudes were elevated at $4.5 \mathrm{~s}$ for negative $(t[80]=2.54, p=.013, d=.09)$ and reminder $(t[80]=2.50, p=$ $.015, d=.08)$ trials, while no differences relative to neutral trials were found at $2.5 \mathrm{~s}$ or $7 \mathrm{~s}$, all $p$ s > .14. Startle potentiation in negative and reminder trials did not differ from each other, irrespective of startle timing ( $p \mathrm{~s}>.169$ ) and correlated with each other, $r>.41, p \mathrm{~s}<.001$. 


\section{References}

American Psychiatric Association. (2000). Diagnostic and Statistical Manual of Mental Disorders: DSM-IV, Text Revision. Washington, DC: Author.

Bennett, I. J., Barnes, K. A., Howard, J. H., \& Howard, D. V. (2009). An abbreviated implicit spatial context learning task that yields greater learning. Behavior Research Methods, 41, 391-395. doi: 10.3758/brm.41.2.391

Bisby, J. A., King, J. A., Brewin, C. R., Burgess, N., \& Curran, H. V. (2010). Acute effects of alcohol on intrusive memory development and viewpoint dependence in spatial memory support a dual representation model. Biological Psychiatry, 68, 280-286.

Blumenthal, T. D., Cuthbert, B. N., Filion, D. L., Hackley, S., Lipp, O. V., \& Van Boxtel, A. (2005). Committee report: Guidelines for human startle eyeblink electromyographic studies. Psychophysiology, 42, 1-15. doi: 10.1111/j.1469-8986.2005.00271.x

Bonanno, G. A. (2004). Loss, trauma, and human resilience - Have we underestimated the human capacity to thrive after extremely aversive events? American Psychologist, 59, 20-28. doi: 10.1037/0003-066x.59.1.20

Bouman, T. K., Luteijn, F., Albersnagel, F. A., \& Van der Ploeg, F. A. G. (1985). Enige ervaringen met de Beck Depression Inventory (BDI) [Some experiences with the Beck Depression Inventory]. Gedrag - Tijdschrift voor Psychologie [Behavior - Journal of Psychology], 13, 13-24.

Bradley, M. M., \& Lang, P. J. (1999). Affective norms for English words (ANEW): Instruction manual and affective ratings. Gainesville, FL: Center for Research in Psychophysiology, University of Florida.

Brewin, C. R., Gregory, J. D., Lipton, M., \& Burgess, N. (2010). Intrusive images in psychological disorders: Characteristics, neural mechanisms, and treatment implications. Psychological Review, 117, 210-232. doi: 10.1037/a0018113

Burgess, N., Maguire, E. A., \& O'Keefe, J. (2002). The human hippocampus and spatial and episodic memory. Neuron, 35, 625-641. doi: 10.1016/s0896-6273(02)00830-9

Chua, K. P., \& Chun, M. M. (2003). Implicit scene learning is viewpoint dependent. Perception \& Psychophysics, 65, 72-80.

Chun, M. M., \& Jian, Y. H. (1998). Contextual cueing: Implicit learning and memory of visual context guides spatial attention. Cognitive Psychology, 36, 28-71. 
Chun, M. M., \& Phelps, E. A. (1999). Memory deficits for implicit contextual information in amnesic subjects with hippocampal damage. Nature Neuroscience, 2, 844-847.

Davis, M., Walker, D. L., \& Lee, Y. L. (1997). Roles of the amygdala and bed nucleus of the stria terminalis in fear and anxiety measured with the acoustic startle reflex - Possible relevance to PTSD. Annals of the New York Academy of Sciences, 821, 305-331. doi: 10.1111/j.1749-6632.1997.tb48289.x

de Quervain, D. J. F., Aerni, A., Schelling, G., \& Roozendaal, B. (2009). Glucocorticoids and the regulation of memory in health and disease. Frontiers in Neuroendocrinology, 30, 358-370. doi: 10.1016/j.yfrne.2009.03.002

Delahanty, D. L., \& Nugent, N. R. (2006). Predicting PTSD prospectively based on prior trauma history and immediate biological responses. In R. Yehuda (Ed.), Psychobiology of Posttraumatic Stress Disorder: A Decade of Progress (Vol. 1071, pp. 27-40). Boston: New York Academy of Science.

Elzinga, B. M., \& Bremner, J. D. (2002). Are the neural substrates of memory the final common pathway in posttraumatic stress disorder (PTSD)? Journal of Affective Disorders, 70, 1-17.

Fan, J., McCandliss, B. D., Sommer, T., Raz, A., \& Posner, M. I. (2002). Testing the efficiency and independence of attentional networks. Journal of Cognitive Neuroscience, 14, 340-347. doi: 10.1162/089892902317361886

Foa, E. B., Riggs, D. S., Dancu, C. V., \& Rothbaum, B. O. (1993). Reliability and validity of a brief instrument for assessing post-traumatic stress disorder. Journal of Traumatic Stress, 6, 459-473.

Francati, V., Vermetten, E., \& Bremner, J. D. (2007). Functional neuroimaging studies in posttraumatic stress disorder: Review of current methods and findings. Depress Anxiety, 24, 202-218. doi: 10.1002/da.20208

Fyhn, M., Hafting, T., Treves, A., Moser, M.-B., \& Moser, E. I. (2007). Hippocampal remapping and grid realignment in entorhinal cortex. Nature, 446, 190-194.

Fyhn, M., Molden, S., Witter, M. P., Moser, E. I., \& Moser, M.-B. (2004). Spatial representation in the entorhinal cortex. Science, 305, 1258-1264. doi: 10.1126/science.1099901

Gilbertson, M. W., Williston, S. K., Paulus, L. A., Lasko, N. B., Gurvits, T. V., Shenton, M. E., . . O Orr, S. P. (2007). Configural cue performance in identical twins discordant for 
posttraumatic stress disorder: Theoretical implications for the role of hippocampal function. Biological Psychiatry, 62, 513-520. doi: 10.1016/j.biopsych.2006.12.023

Hagenaars, M. A., Brewin, C. R., van Minnen, A., Holmes, E. A., \& Hoogduin, K. A. L. (2010). Intrusive images and intrusive thoughts as different phenomena: Two experimental studies. Memory, 18, 76-84. doi: 10.1080/09658210903476522

Holmes, E. A., Brewin, C. R., \& Hennessy, R. G. (2004). Trauma films, information processing, and intrusive memory development. Journal of Experimental PsychologyGeneral, 133, 3-22. doi: 10.1037/0096-3445.133.1.3

Holmes, E. A., James, E. L., Coode-Bate, T., \& Deeprose, C. (2009). Can playing the computer game "Tetris" reduce the build-up of flashbacks for trauma? A proposal from cognitive science. Plos One, 4, 6. doi: e415310.1371/journal.pone.0004153

Holmes, E. A., \& Mathews, A. (2010). Mental imagery in emotion and emotional disorders. Clinical Psychology Review, 30, 349-362. doi: 10.1016/j.cpr.2010.01.001

Horowitz, M. J., Wilner, N., \& Alvares, W. (1979). Impact of Event Scale: A measure of subjective stress. Psychosomatic medicine, 41, 209-218.

Jackson, D. C., Mueller, C. J., Dolski, I., Dalton, K. M., Nitschke, J. B., Urry, H. L., . . . Davidson, R. J. (2003). Now you feel it, now you don't: Frontal brain electrical asymmetry and individual differences in emotion regulation. Psychological Science, $14,612-617$.

Jordan, K., Heinze, H. J., Lutz, K., Kanowski, M., \& Jancke, L. (2001). Cortical activations during the mental rotation of different visual objects. NeuroImage, 13, 143-152.

Lang, P. J., Bradley, M. M., \& Cuthbert, B. N. (2005). International Affective Picture System (IAPS): Instruction Manual and Affective Ratings. Technical Report A-6. Gainesville, FL: University of Florida.

Lleras, A., \& Von Muhlenen, A. (2004). Spatial context and top-down strategies in visual search. Spatial Vision, 17, 465-482.

Manns, J. R., \& Squire, L. R. (2001). Perceptual learning, awareness, and the hippocampus. Hippocampus, 11, 776-782. doi: 10.1002/hipo.1093

Marmar, C. R., McCaslin, S. E., Metzler, T. J., Best, S., Weiss, D. S., Fagan, J., . . Neylan, T. (2006). Predictors of posttraumatic stress in police and other first responders. In R. Yehuda (Ed.), Psychobiology of posttraumatic stress disorder: A decade of progress (pp. 1-18). Boston: New York Academy of Science. 
Murray, E. A., Bussey, T. J., \& Saksida, L. M. (2007). Visual perception and memory: A new view of medial temporal lobe function in primates and rodents. Annual Review of Neuroscience, 30, 99-122. doi: 10.1146/annurev.neuro.29.051605.113046

Orr, S. P., \& Roth, W. T. (2000). Psychophysiological assessment: Clinical applications for PTSD. Journal of Affective Disorders, 61, 225-240.

Ozer, E. J., Best, S. R., Lipsey, T. L., \& Weiss, D. S. (2003). Predictors of posttraumatic stress disorder and symptoms in adults: A meta-analysis. Psychological Bulletin, 129, 52-73. doi: 10.1037//0033-2909.129.1.52

Preston, A. R., \& Gabrieli, J. D. E. (2008). Dissociation between explicit memory and configural memory in the human medial temporal lobe. Cerebral Cortex, 18, 21922207. doi: 10.1093/cercor/bhm245

Rubin, D. C., Berntsen, D., \& Bohni, M. K. (2008). Memory-based model of posttraumatic stress disorder: Evaluating basic assumptions underlying the PTSD diagnosis. Psychological Review, 115, 985-1011. doi: 10.1037/a0013397

Rubin, D. C., Boals, A., \& Berntsen, D. (2008). Memory in posttraumatic stress disorder: Properties of voluntary and involuntary, traumatic and nontraumatic autobiographical memories in people with and without posttraumatic stress disorder symptoms. Journal of Experimental Psychology-General, 137, 591-614. doi: 10.1037/a0013165

Shin, L. M., Rauch, S. L., \& Pitman, R. K. (2006). Amygdala, medial prefrontal cortex, and hippocampal function in PTSD. In R. Yehuda (Ed.), Psychobiology of posttraumatic stress disorder: A decade of progress (Vol. 1071, pp. 67-79). Boston: New York Academy of Science.

Steil, R., \& Ehlers, A. (2000). Dysfunctional meaning of posttraumatic intrusions in chronic PTSD. Behaviour Research and Therapy, 38, 537-558. doi: 10.1016/s00057967(99)00069-8

Suvak, M. K., \& Barrett, L. F. (2011). Considering PTSD from the perspective of brain processes: A psychological construction approach. Journal of Traumatic Stress, 24, 324. doi: $10.1002 /$ jts.20618

Thayer, J. F., Ahs, F., Fredrikson, M., Sollers, J. J., \& Wager, T. D. (2012). A meta-analysis of heart rate variability and neuroimaging studies: Implications for heart rate variability as a marker of stress and health. Neurosci Biobehav Rev, 36, 747-756. doi: 10.1016/j.neubiorev.2011.11.009 
Van der Ploeg, H. M. (1985). The development and validation of the Dutch State-Trait Anxiety Inventory. In C. D. Spielberger \& I. G. Sarason (Eds.), Stress and Anxiety (pp. 129-139). Washington, DC: Hemisphere.

van Strien, N. M., Cappaert, N. L. M., \& Witter, M. P. (2009). The anatomy of memory: An interactive overview of the parahippocampal-hippocampal network. Nature Reviews Neuroscience, 10, 272-282. doi: 10.1038/nrn2614

Watson, D., Clark, L. A., \& Tellegen, A. (1988). Development and validation of brief measures of positive and negative affect: The PANAS scales. Journal of Personality and Social Psychology, 54, 1063-1070.

\section{Acknowledgements}

This study was supported in part by Grant 056-25-011 from the Netherlands Organization for Scientific Research (NWO) to Dr. Tom Smeets. The authors declare that no conflict of interest exists. We are especially thankful to Ron Hellenbrand, Jacco Ronner, and Felix Dücker (Maastricht University) for implementing the experimental paradigms, to Prof. Emily Holmes and Ella James (University of Oxford) for providing the materials for the trauma film paradigm, as well as to Dr. Ilana Bennett and her former colleagues from Georgetown University for providing their SCCT script and instructions that served as the basis for our study. 



\section{Chapter 3}

\section{Acute stress differentially affects spatial configuration learning in high and low cortisol-responding healthy adults}

Published as:

Meyer, T., Smeets, T., Giesbrecht, T., Quaedflieg, C. W. E. M., \& Merckelbach, H. (2013). Acute stress differentially affects spatial configuration learning in high and low cortisolresponding healthy adults. European Journal of Psychotraumatology, 4. 



\section{Abstract}

Background: Stress and stress hormones modulate memory formation in various ways that are relevant to our understanding of stress-related psychopathology, such as posttraumatic stress disorder (PTSD). Particular relevance is attributed to efficient memory formation sustained by the hippocampus and parahippocampus. This process is thought to reduce the occurrence of intrusions and flashbacks following trauma, but may be negatively affected by acute stress. Moreover, recent evidence suggests that the efficiency of visuo-spatial processing and learning based on the hippocampal area is related to PTSD symptoms. Objective: The current study investigated the effect of acute stress on spatial configuration learning using a spatial contextual cueing task (SCCT) known to heavily rely on structures in the parahippocampus. Method: Acute stress was induced by subjecting participants $(N=34)$ to the Maastricht Acute Stress Test (MAST). Following a counterbalanced within-subject approach, the effects of stress and the ensuing hormonal (i.e., cortisol) activity on subsequent SCCT performance were compared to SCCT performance following a no-stress control condition. Results: Acute stress did not impact SCCT learning overall, but opposing effects emerged for high versus low cortisol responders to the MAST. Learning scores following stress were reduced in low cortisol responders, while high cortisol-responding participants showed improved learning. Conclusions: The effects of stress on spatial configuration learning were moderated by the magnitude of endogenous cortisol secretion. These findings suggest a possible mechanism by which cortisol responses serve an adaptive function during stress and trauma, and this may prove to be a promising route for future research in this area.

\section{Keywords}

Maastricht Acute Stress Test; contextual cueing paradigm; spatial memory; hippocampal area; posttraumatic stress disorder 


\section{Introduction}

Acute stress and stress-related hormonal activity profoundly influence learning and memory. Such stress-induced memory alterations have attracted the attention of researchers, many of whom believe that they typically serve adaptive purposes but can also represent key mechanisms in the development of highly prevalent emotional disorders (de Kloet, Joels, \& Holsboer, 2005; de Quervain, Aerni, Schelling, \& Roozendaal, 2009; Joels, 2011; Wingenfeld $\&$ Wolf, 2011). Indeed, different mood and anxiety disorders appear to be characterized by abnormal memory function, such as enhanced learning, consolidation, or retrieval of negative information (de Quervain et al., 2009; Wolf, 2008), as well as more distressing involuntary recollections (Brewin, Gregory, Lipton, \& Burgess, 2010). In posttraumatic stress disorder (PTSD), stress-related changes in memory function are of particular relevance, as this disorder may develop after highly stressful events and is characterized by recurrent flashbacks and an apparent inability to integrate these aversive memories with other autobiographical memories (Brewin et al., 2010).

Based on studies investigating stress effects on learning in rodents (e.g., Schwabe, Schachinger, de Kloet, \& Oitzl, 2010) and humans (e.g., Schwabe et al., 2007), Schwabe and colleagues (Schwabe, Wolf, \& Oitzl, 2010) recently suggested that stress may reduce "cognitive" memory formation in the hippocampal area (e.g., spatial navigation learning) in favor of increased reliance on "habit" memory (e.g., associations based on stimulus-response). Interestingly, these findings accord well with a recent theory on the development of intrusions (Brewin et al., 2010) proposing that intrusions of stressful experiences occur when a memory system in the hippocampal area fails to construct contextualized representations of that event (i.e., possibly as a consequence of stress). Therefore, investigating the effects of stress on memory formation in the hippocampal area may provide further theoretical insights with relevance for stress and trauma research.

In humans, this line of research has focused almost exclusively on (declarative) memory of words or pictures (for review, see Schwabe, Wolf, et al., 2010), whereas little attention has been given to stress effects on visuospatial memory until very recently (Taverniers et al., 2011; Taverniers, Taylor, \& Smeets, 2013). Nevertheless, some studies indicate that visuospatial learning, based on the hippocampal area, is relevant to PTSD symptoms. For instance, PTSD patients displayed impaired spatial configuration processing, a deficiency that was statistically predictive of PTSD symptom severity (Gilbertson et al., 2007). Another study using trauma films in healthy individuals found that view-point independent spatial 
recognition performance predicted fewer intrusions (Bisby, King, Brewin, Burgess, \& Curran, 2010). In light of theories suggesting reduced hippocampal-area based memory under stress, and a role of this brain region in intrusions (cf., supra), these findings imply that stress and stress hormone responding could be related to intrusions by impairing visuo-spatial learning efficiency.

In accordance with the Gilbertson et al. (2007) and Bisby et al. (2010) studies, a recent study by Meyer et al. (2013) demonstrated that intrusions after viewing a trauma film were related to worse implicit spatial configuration learning in a contextual cueing paradigm (Chun $\&$ Jian, 1998). This paradigm measures the degree to which spatial configurations of multiple simple cues are bound in memory, which depends crucially on structures in the medial temporal lobe (Chun \& Phelps, 1999; Manns \& Squire, 2001). An fMRI study in healthy participants (Preston \& Gabrieli, 2008) has indicated that this implicit learning performance relies on structures in the parahippocampus, which are thought to serve as a major input for the construction of spatial representations in the hippocampus (Fyhn, Hafting, Treves, Moser, $\&$ Moser, 2007). Although the exact relationship between contextual cueing performance and intrusions remains to be clarified, visuo-spatial learning appears to reflect information processing that is adaptive during a traumatic event. The current study addresses whether this performance is affected by stress and stress hormone responses.

To test this, we compared performance on a contextual cueing paradigm under stress and no-stress control conditions. Specifically, healthy individuals were subjected to the Maastricht Acute Stress Test (MAST; Smeets et al., 2012) and to a no-stress version of the MAST in a counterbalanced within-subject cross-over design, measuring subjective and hormonal (i.e., cortisol) responses on each test occasion. Both conditions were followed by the administration of a contextual cueing task, allowing us to assess the effects of stress versus control condition within subjects.

To our knowledge, this is the first human study to address stress effects on implicit visuo-spatial learning known to rely on parahippocampal structures. In contrast, prior studies focused on word or picture learning (often finding memory facilitation when stress targets the learning phase; for a review, see Schwabe, Wolf, et al., 2010) or on explicit spatial memory performance (finding memory impairments when stress targets the learning phase; Taverniers et al., 2013). Given the scarcity of prior research, it was not possible a priori to formulate firm hypotheses. However, based on theoretical considerations (cf., supra), we expected contextual cueing performance to be worse in the stress condition as a result of heightened glucocorticoid responses. Since the literature suggests possible gender differences in the 
effect of stress on memory (Smeets, Dziobek, \& Wolf, 2009; Wolf, Schommer, Hellhammer, McEwen, \& Kirschbaum, 2001), we tested this in a balanced sample of men and women.

\section{Method}

\section{Participants}

A total of 34 healthy participants (50\% women), recruited at Maastricht University campus, completed this study. Mean age was $21.4(S D=3.4$, range $=18-36)$. Eligibility was checked using a screening form, exclusion criteria being a body mass index (BMI; $\mathrm{kg} / \mathrm{m}^{2}$ ) below 18 or above 30, cardiovascular disease, severe physical illness, endocrine disorders, current psychopathology, substance abuse, heavy smoking (> 10 cigarettes/day), and current use of medication known to affect the function of the hypothalamic-pituitary-adrenal (HPA) axis. For women, hormonal contraceptive use was required as an inclusion criterion, as this is known to suppress cortisol response variation due to the menstrual cycle (Kudielka, Hellhammer, \& Wüst, 2009). The standing ethical committee of the Faculty of Psychology and Neuroscience, Maastricht University, approved this study. All participants gave written informed consent and were compensated with a small financial reward or partial course credit in return for their participation.

\section{Maastricht Acute Stress Test}

The MAST (Smeets et al., 2012) is an effective stress induction procedure that combines physical stress with uncontrollability, unpredictability, social-evaluative, and mental arithmetic elements to produce reliable subjective and cortisol stress responses. The duration of the MAST is 15 min. Participants first undergo a 5-min preparation phase in which instructions about the MAST procedure are presented on a computer screen. In the following 10-min acute stress phase, they are alternately prompted by instructions on the computer screen to immerse their hand in ice-cold water $\left(28^{\circ} \mathrm{C}\right)$ or to engage in a mental arithmetic test (counting backwards from 2043 in steps of 17). During mental arithmetic trials, participants are additionally asked to direct their gaze toward a video camera (enabling them to see themselves on a TV monitor) and receive negative performance feedback by the experimenter concerning accuracy and/or speed of the calculations. In the current study, five hand immersion trials (duration: 60 or $90 \mathrm{sec}$ ) were alternated with four mental arithmetic trials (duration: $45-90 \mathrm{sec}$ ), while participants were unaware of the number and exact duration of the two types of trials. 


\section{No-stress control condition}

The procedure of the no-stress control condition was identical to the MAST, except that all stressful elements were removed (see Smeets et al., 2012; Experiment 3). That is, there was no videotaping and the water was lukewarm $\left(35^{\circ} \mathrm{C}\right)$. The mental arithmetic test was replaced by instructions to count aloud consecutively from 1 to 25 at a self-chosen pace and to start again at 1 when having reached 25 . The experimenter checked participants' compliance but provided no feedback on their performance.

\section{Assessment of stress responses}

Mood changes in response to the MAST and control condition were measured using repeated administrations of the Positive and Negative Affect Schedule, state version (PANAS; Watson, Clark, \& Tellegen, 1988), consisting of two 10-item subscales for positive affect (PA; all Cronbach's $\alpha \mathrm{s}>0.88$ ) and negative affect (NA; all Cronbach's $\alpha \mathrm{s}>0.69$ ). Items refer to current mood (e.g., PA: interested, NA: distressed) and are rated on five-point scales $(1=$ very slightly or not at all; $5=$ very much $)$. Salivary cortisol measurements were taken as a measure of hormonal stress responding of the HPA axis using synthetic Salivette devices (Sarstedt ${ }^{\circledR}$, Etten-Leur, the Netherlands) at five time points in each session. The first measurement was taken immediately before MAST or control condition onset (i.e., $15 \mathrm{~min}$ before stress offset; tpre-stress) and four times relative to the end of the MAST or control condition $\left(t_{+00}, t_{+10}, t_{+25}\right.$, and $t_{+40}$; see Figure 3.1). On collection, samples were stored at $-20{ }^{\circ} \mathrm{C}$ immediately. Cortisol levels were determined by a commercially available luminescence immuno assay (IBL, Hamburg, Germany). Mean intra- and inter-assay coefficients of variation are typically less than 8 and $12 \%$, respectively, and the lower and upper detection limits were $0.015 \mathrm{mg} / \mathrm{dl}(0.41 \mathrm{nmol} / \mathrm{l})$ and $4.0 \mathrm{mg} / \mathrm{dl}(110.4 \mathrm{nmol} / \mathrm{l})$, respectively.

\section{Spatial Contextual Cueing Task}

In the contextual cueing paradigm (Chun \& Jian, 1998), participants are required to find a single target ("T"'-shaped symbol rotated $90^{\circ}$ or $270^{\circ}$ ) among 11 rotated "L"-shaped distracters (i.e., the visuo-spatial context of the target). In half of the trials, the configuration of distracter stimuli is repeated, while in the other half of the trials new distracter configurations are presented. The repeated target contexts predict the location of the target, thereby facilitating search, as evidenced by faster reaction times (RTs) compared to new 
distracter configurations. This RT difference is a measure of the contextual learning effect (Chun \& Jian, 1998), with higher values reflecting a stronger learning effect.

Similar to Meyer et al. (2013), we used the abbreviated Spatial Contextual Cueing Task (SCCT) developed by Bennett and colleagues (2009). Each SCCT administration consisted of 30 blocks with 12 trials, 6 of which had repeated arrays, while 6 had novel arrays. In addition, 12 trials with new arrays were presented along with task instructions as a separate training block before the actual task. All arrays were computed individually for each participant and session. Trials started with a $1 \mathrm{~s}$ fixation period, followed by a configuration display that required participants to indicate as quickly and accurately as possible whether the base of the target stimulus pointed left or right by pressing response keys on a right-hand response box. The configuration display was presented for $10 \mathrm{sec}$ or until the participant responded. Before the next trial started, auditory feedback was provided upon response (a high-pitch or lowpitch tone for correct or incorrect/too slow responses, respectively). Each block was followed by a break that could be ended by the participant. After block 15, there was a forced 2-min break for the collection of a saliva cortisol probe.

For data reduction, median RTs of accurate trials were derived for each of the 30 blocks, and separately for each array type (novel and repeated). These median RTs were subsequently averaged across five consecutive blocks, respectively, yielding novel and repeated RT scores of six epochs. For each of the six epochs, one learning score was then calculated by subtracting repeated RT scores from novel RT scores. In addition, accuracy scores were calculated for the six epochs, and separately for array type.

\section{Procedure}

Participants were invited to two lab sessions separated by a 1-week interval. Each session took place in the afternoon between 12:30 and 18:00 $\mathrm{h}$ to control for circadian cortisol rhythms (Nicolson, 2008). Before each session, participants were instructed by email to come well-rested, to refrain from consuming alcohol or drugs the evening before participation, and to refrain from other activities known to affect cortisol measurements immediately before participation (e.g., eating, smoking, heavy physical activity, brushing teeth). Adherence to these rules was checked upon arrival, and in cases of violation the session was rescheduled (this applied to only one participant). Next, participants were subjected to either the MAST or the control condition, preceded also by administration of the PANAS and a salivary cortisol probe. Participants were then given SCCT instructions and one SCCT training block. 
Participants performed the SCCT, with cortisol probes being taken before, midway through, and after task administration. Finally, the PANAS was administered a third time. The procedure of the second session was similar to the first one, except that MAST and a control condition were substituted (see Figure 3.1; with counterbalanced order of MAST and control condition across participants).

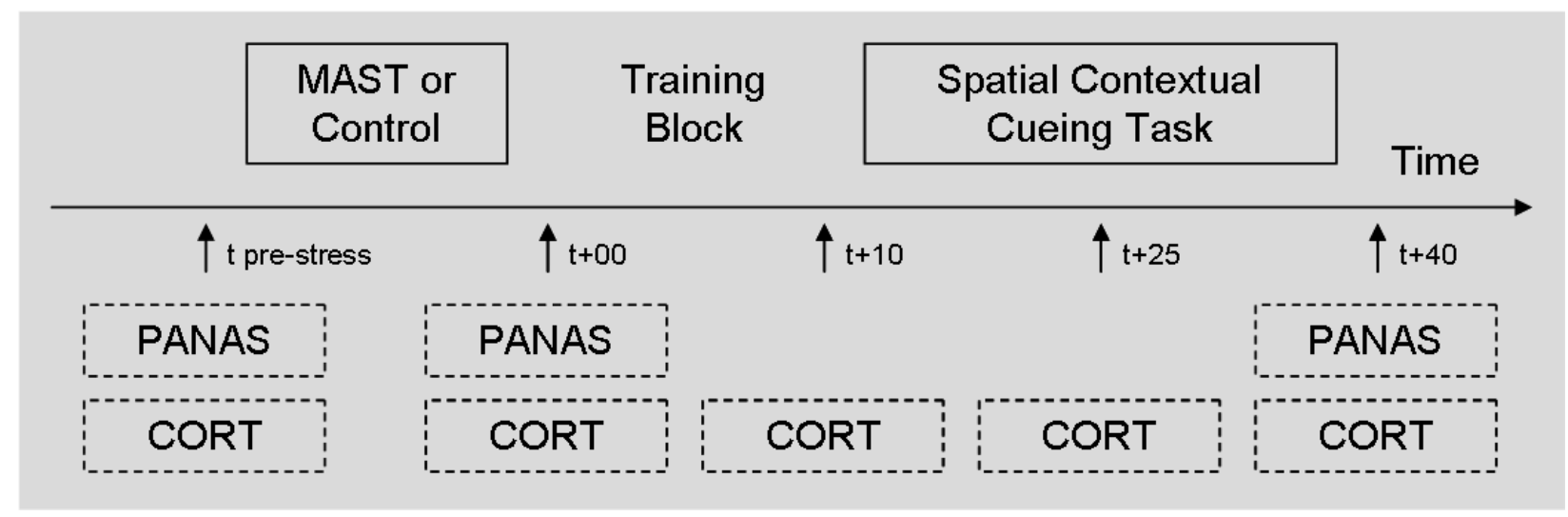

Figure 3.1. Overview of the procedure. The procedure was similar for session 1 and session 2, the crucial difference being the stress vs. control condition. Abbreviations: MAST, Maastricht Acute Stress Test; PANAS, Positive and Negative Affect Schedule; CORT, cortisol sample.

\section{Statistical analysis}

Affective (i.e., PA and NA) and cortisol responding were analysed using repeated measures ANOVAs with time point of measurement and condition (MAST, control) as within-subjects factors. To explore gender effects, sex was additionally entered as betweensubjects factor, and where appropriate, follow-up tests and post-hoc pairwise comparisons with Bonferroni adjustment were used to explore interaction effects. In addition, delta-peak cortisol values from the stress condition were used to compare the magnitude of cortisol responding between males and females, using an independent-samples $t$-test. The effect of condition on SCCT learning scores was analysed using repeated measures ANOVA with epoch and condition as within-subjects factors and gender as between-subjects factor. To assess whether possible reductions in contextual cueing performance can be accounted for by glucocorticoid responses in the stress condition, cortisol responding was entered by creating groups of low and high cortisol responders based on delta-peak cortisol values relative to prestress (group allocation by median-split). Also, we tested a linear association between deltapeak cortisol values and mean SCCT learning scores of the stress condition using correlational analysis. SCCT accuracy scores were not included in the analyses because they 
were too close to ceiling in all arrays, epochs, and conditions (all means > 97\%). When the assumption of non-sphericity was violated in the data, Greenhouse-Geisser corrected degrees of freedom and $p$-values are reported. Alpha was set at 0.05 for all tests.

\section{Results}

\section{Cortisol responses}

A 2 (Condition: stress, control) by 5 (Time: cortisol measurements) by 2 (Gender) repeated measures ANOVA revealed a significant three-way interaction of Condition by Time by Gender, $F(1.9,59.9)=4.7, p=0.014, \eta p 2=0.13$. Separate follow-up tests for men and women revealed significant Condition by Time interactions in both men, $F(2.1,33.3)=23.8$, $p<0.001, \eta p 2=0.60$ and women, $F(1.5,24.2)=6.2, p=0.011, \eta p 2=0.28$. Post-hoc pairwise comparisons showed that men displayed elevated cortisol levels in the stress condition, as compared with the control condition, at $t_{+10}, t_{+25}$, and $t_{+40}$ (Bonferroni-adjusted $p \mathrm{~s}<0.005$ ), but not at $t_{\text {pre-stress }}$ or $t_{+00}$ (adjusted $p \mathrm{~s}>0.115$ ). Relative to the control condition, women displayed elevated cortisol levels in the stress condition only at $t_{+10}$ and $t_{+25}$ (adjusted $p \mathrm{~s}<0.036$ ), but not at $t_{\text {pre-stress }}, t_{+00}$, or $t_{+40}$ (adjusted $p \mathrm{~s}>0.118$ ). An independent-samples $t$ test comparing delta-peak cortisol levels in the stress condition between men and women revealed a trend toward stronger cortisol increases in men $(M=14.5 \mathrm{nmol} / \mathrm{l}, S D=9.5)$ than in women $(M=8.5 \mathrm{nmol} / 1, S D=9.2), t(32)=1.9, p=0.069$. Descriptively, $88 \%$ of men $(15 / 17)$ and $65 \%$ of women (11/17) could be classified as cortisol responders in the MAST condition (i.e., displaying a cortisol increase > $2.5 \mathrm{nmol} / \mathrm{l}$; e.g., Kirschbaum, Pirke, \& Hellhammer, 1993), Pearson Chi-square $=2.62, p=0.106$. Cortisol data are summarized visually in Figure 3.2.

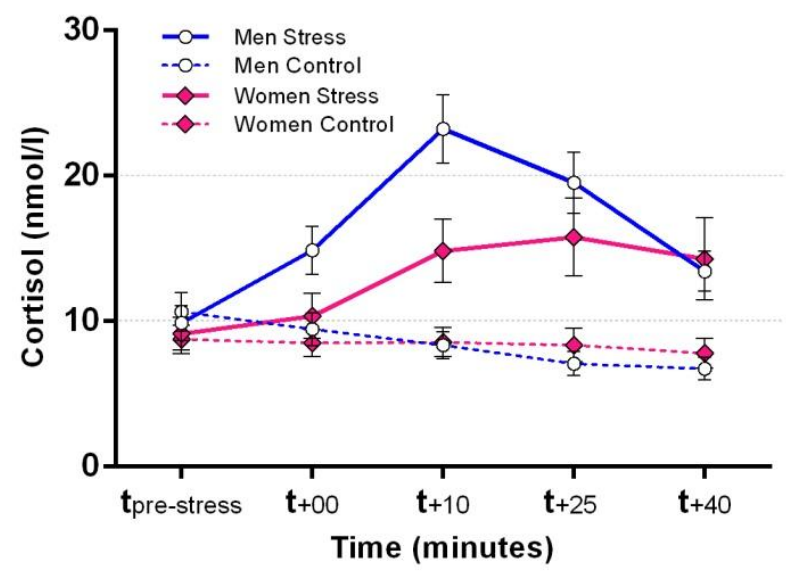

Figure 3.2. Cortisol responses in men and women to the MAST vs. control condition. Endogenous cortisol responses were robust in both men and women using hormonal contraceptives; Error bars represent standard errors of measurement. 


\section{Mood responses}

A 2 (Condition: stress, control) by 3 (Time: PANAS measurements) by 2 (Gender) repeated measures ANOVA for NA revealed a significant Condition by Time interaction, $F$ $(1.4,45.4)=14.2, p<0.001, \eta p 2=0.31$, in the absence of main or interactive effects involving Gender (all $F \mathrm{~s}<2.3$, all $p \mathrm{~s}>0.143$ ). Post-hoc pairwise comparisons indicated that NA levels were elevated in the stress condition, as compared with the control condition, at pre-stress, $t(33)=2.6$, Bonferroni-adjusted $p=0.042$, and at post-stress, $t(33)=4.6$, adjusted $p<0.001$. NA levels did not differ between conditions at the end of the session, $t(33)=0.9$, $n s$. For PA, the 2 by 3 by 2 repeated measures ANOVA revealed a main effect of Time, $F(2$, $64)=24.2, p<0.001, \eta p 2=0.43$, that did not interact with Condition, Gender, or both (all $F \mathrm{~s}$ $<1.6$, all $\mathrm{ps}>0.22$ ). Post-hoc pairwise comparisons showed that in both conditions, PA decreased from pre- to post-stress, $t \mathrm{~s}(33)>3.0$, Bonferroni adjusted $p \mathrm{~s}<0.002$, and remained stable afterwards, $t(33)<2.2$, Bonferroni adjusted $p$ s $>0.127$.

\section{Stress effects on SCCT performance ${ }^{l}$}

A 2 (Condition: stress, control) by 6 (Epoch) by 2 (Gender: male, female) repeated measures ANOVA on SCCT learning scores revealed a significant main effect of Epoch, $F(3.2,103.5)=10.1, p<0.001, \eta p 2=0.24$, with contextual learning scores increasing across epochs. This learning effect did not interact with Condition, $F(4,129.3)=0.73, p=0.58, \eta p 2$ $=0.02$, Gender, $F(3.2,103.5)=2.3, p=0.08, \eta p 2=0.07$, or both, $F(4,129.3)=1.1, p=0.38$, $\eta p 2=0.03$. Neither were there main effects of Condition, $p=0.90$, or Gender, $F(1,32)=1.5$, $p=0.22, \eta p 2=0.05$. Overall, average SCCT learning scores differed significantly from zero $($ Grand Mean $=181.9 \mathrm{~ms} ; S E=42.0), F(1,32)=18.7, p<0.001, \eta p 2=0.37$, reflecting the typical contextual cueing effect.

1 The procedural order of Condition (MAST first vs. control condition first) had no significant main or interaction effects on SCCT learning and was therefore not included in subsequent analyses. 
To assess the specific role of cortisol responding, delta-peak cortisol values were entered as a two-level factor (group allocation by median-split) ${ }^{2}$. A 2 (Condition: stress, control) by 6 (Epoch) by 2 (Responder: high, low) repeated measures ANOVA showed a significant Condition by Responder interaction, $F(1,32)=9.2, p<0.005, \eta p 2=0.22$, in the absence of a three-way interaction, $F(3.9,126)=0.9, p=0.46$. Examination of this effect separately for each condition showed that the responder groups differed from each other in the stress, $F(1,32)=8.1, p=0.008, \eta p 2=0.203$, but not the control condition, $F(1,32)=1.9, p=$ $0.177, \eta p 2=0.06$. Follow-up tests suggest a negative effect of the stress condition as compared with the control condition on SCCT learning in low cortisol responders, $F(1,16)=$ 4.9 , uncorrected $p=0.042, \eta p 2=0.23$, and a positive effect of the stress condition in high cortisol responders, $F(1,16)=4.4$, uncorrected $p=0.052, \eta p 2=0.22$ (see Figure 3.3). In line with these findings, delta-peak cortisol values in the stress condition correlated positively with SCCT learning scores in the stress condition, $r=0.353, p=0.042$ (two-tailed).

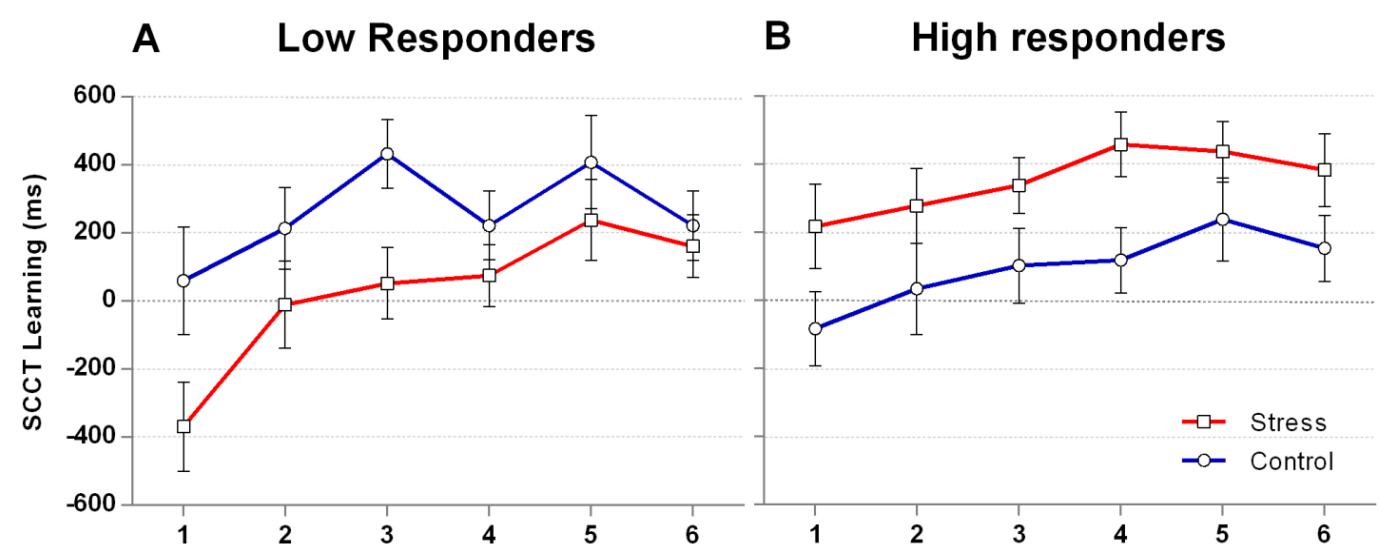

Figure 3.3. Contextual cueing effect across time for low and high cortisol responders. $N=17$ in each group. SCCT Learning scores reflect the RT difference on trials with novel and repeated arrays, respectively, with higher values indicating better learning. Error bars represent standard errors of measurement.

2 Using area under the curve with respect to increase (AUC_i; Pruessner, Kirschbaum, Meinlschmid, \& Hellhammer, 2003) for Responder group allocation yielded nearly identical groups and results. 


\section{Discussion}

The present study explored the effects of stress and stress-related cortisol secretion on implicit spatial configuration learning in humans. We used a contextual cueing paradigm that requires a type of learning that is thought to depend crucially on the parahippocampal region (Chun \& Phelps, 1999; Manns \& Squire, 2001; Preston \& Gabrieli, 2008). In a counterbalanced within-subject cross-over design, participants were subjected to the MAST (Smeets et al., 2012) and to a no-stress control condition, each condition followed by administration of the SCCT (Bennett et al., 2009). Based on the proposition that hippocampalarea based memory may be reduced under stress (Schwabe, Wolf, et al., 2010), we hypothesized that stress would undermine implicit spatial configuration learning.

Demonstrating the effectiveness of our stress task, we observed both subjective (in terms of NA increases from pre- to post-stress) and hormonal (in terms of salivary cortisol increases) stress responses in the MAST condition, but not in the control condition. In both sessions, participants displayed the contextual cueing effect (i.e., faster RTs on trials with repeated vs. novel arrays), as well as an increase of this effect in the course of the task, which is typical for this paradigm (Bennett et al., 2009; Chun \& Jian, 1998). Our data also suggest that SCCT administration itself did not affect stress responding, since the SCCT was neither accompanied by cortisol increases nor by negative mood responses in the control condition.

Contrary to our expectations, there was no overall effect of the stress condition on the contextual cueing effect or on learning across epochs, compared to the control condition. There also was no evidence suggesting that the effect of stress differed between men and women, which adds to a literature with mixed results with respect to gender differences (Smeets et al., 2009; Wolf et al., 2001). When taking cortisol secretion into account, however, opposing effects of the stress condition emerged for high versus low responders, which turned out to account for the absence of an overall condition effect in the sample. In particular, our data suggest that only participants with low cortisol secretion (including non-responders) have reduced overall SCCT learning scores after stress, whereas in participants with higher cortisol secretion, our data suggest a trend towards amplified learning scores after stress. In support of this interpretation, we found a significant positive correlation between cortisol responding and mean SCCT learning scores in the stress condition.

Importantly, in both low and high cortisol responders, stress appeared to have main effects on SCCT learning scores (i.e., diminishing or amplifying the overall contextual cueing effect), whereas the increase of learning scores over time was unaffected by stress in both 
responder groups. This indicates that stress had an effect on an early stage of implicit spatial memory formation, which was apparently moderated by endogenous cortisol secretion, with continued visuo-spatial learning in the course of the task apparently remaining unaffected. Although studies using the contextual cueing paradigm typically do not distinguish between stages of implicit spatial memory formation (e.g., Chun \& Jian, 1998), these findings seem to suggest that initial encoding of spatial configurations can be modulated independently from further consolidation of these acquired memories. It has been argued that stress can affect consolidation (in long-term declarative memory) through influencing the degree of rehearsal (e.g., Tollenaar, Elzinga, Spinhoven, \& Everaerd, 2008). Because the contextual cueing paradigm inherently controls the number of occasions at which repeated spatial configurations are rehearsed (i.e., each repeated array is presented exactly 30 times), this could be the reason why no stress effects on further consolidation of the contextual cueing effect were found.

Our findings do not support the hypothesis that acute stress impairs implicit spatial configuration learning in general, but point to a specific moderating role of endogenous cortisol secretion. Notably, with respect to explicit memory, differential effects of stress, depending on cortisol responding, have been reported (see, e.g., Domes, Heinrichs, Reichwald, \& Hautzinger, 2002; Smeets et al., 2009). These cortisol-dependent opposite effects of stress on implicit visuo-spatial learning may have implications for theories of stress and stress-related psychopathology. For instance, the prediction of reduced reliance on "cognitive"' (including declarative spatial) learning in the hippocampal area as a consequence of cortisol increases (Schwabe, Wolf, et al., 2010) apparently does not translate to a reduced contextual cueing effect under stress. Instead, we found that cortisol secretion protected or even amplified learning. This might indicate that cortisol differentially affects different systems in the hippocampal area that subserve spatial memory formation. In particular, previous human studies have largely addressed stress effects on declarative memory for which the hippocampus is crucial (Schwabe, Wolf, et al., 2010), whereas the contextual cueing critically depends on structures in the parahippocampus (Chun \& Phelps, 1999; Manns \& Squire, 2001), notably the entorhinal cortex (Preston \& Gabrieli, 2008). These regions serve as major input to the hippocampus for the construction of spatial representations (Fyhn et al., 2007) and may thus display differential responses to stress. However, it is also possible that even more extreme levels of cortisol secretion (e.g., in response to strenuous Special Forces exercises; Taverniers et al., 2013) would lead to lowered performance in the contextual cueing paradigm, as hormonal stress effects have often been hypothesized to follow an inverted-U quadratic function (e.g., Abercrombie, Kalin, Thurow, Rosenkranz, \& Davidson, 2003). 
Interestingly, several studies have reported reduced resting (i.e., basal) cortisol concentrations in PTSD patients, and a link between lowered cortisol and the development and maintenance of intrusions has been suggested (for a review, see Wingenfeld \& Wolf, 2011). In line with this, administration of low doses of cortisol has been shown to be a promising treatment option in PTSD patients (e.g., de Quervain \& Margraf, 2008). Since cortisol elevations during delayed retrieval typically impair declarative memory performance (Schwabe, Wolf, et al., 2010), hypocortisolism in PTSD has been argued to result in weaker inhibition of trauma memories and, hence, to cause more intrusions (Wingenfeld \& Wolf, 2011; though note that lowered resting cortisol does not necessarily imply smaller cortisol stress responses). Our results, however, revealed that strongly enhanced cortisol secretion during stress dampens or even reverses the negative effects of stress on spatial configuration learning. This might reflect a different mechanism by which cortisol responding has adaptive consequences under stress. In addition to inhibiting the retrieval of trauma memories, cortisol might enhance adaptive information processing associated with spatial configuration learning. Relevant to this, Chapter 2 found that superior performance on the SCCT was negatively correlated with intrusions in healthy participants who had viewed a trauma film. Although it is not yet clear in what way implicit visuo-spatial learning would help to reduce intrusions, it might thus reflect information processing that is relevant to the formation of contextualized trauma memories. In this way, stronger cortisol responses during stressful experiences might help to integrate the trauma in autobiographical memory and prevent intrusions, which could be a promising avenue for future research.

\section{Limitations}

The current study had some limitations that deserve to be mentioned. To begin with, our sample consisted entirely of young healthy adults, and it is not clear whether our findings would apply to other populations. Also, although the MAST is a relatively robust experimental stressor (Smeets et al., 2012), the effects may not be comparable to real-life traumatic stressors, implying that these findings may not directly translate to PTSD patients per se. Another limitation is that the design of this study allows no conclusions about differential effects of stress on encoding and consolidation of spatial configurations on the one hand, and on retrieval on the other hand. That is, the repetition of configurations in the SCCT can, by definition, invoke all three of these processes simultaneously. However, it might be possible in future studies to disentangle the effects of stress on encoding and retrieval by 
delivering a delayed test that focuses on a long-term component of implicit spatial memory (e.g., Chun \& Jiang, 2003). Finally, although our findings suggest a specific moderating role of endogenous cortisol secretion in the effects of stress on spatial configuration learning, we are not able to infer whether this role is causal or merely correlational. Therefore, future studies are required to test the possible causal involvement of cortisol by experimentally manipulating hormonal responding (e.g., using pharmacological interventions).

\section{Conclusions}

A large body of evidence shows that stress and stress hormones affect hippocampal-area based memory in various ways that are relevant to our understanding of stress-related psychopathology, including PTSD (de Kloet et al., 2005; Joels, 2011; Wingenfeld \& Wolf, 2011). Recent evidence shows that human spatial processing and learning is also affected by stress (Taverniers et al., 2013) and might be involved in PTSD symptoms (Bisby et al., 2010; Gilbertson et al., 2007; Meyer et al., 2013). The current study demonstrates that stress affects implicit visuo-spatial learning relying on structures in the parahippocampus, whereby the level of endogenous cortisol secretion appeared to moderate the effect of stress on learning performance. The memory-enhancing role of higher cortisol levels on this memory system during stress may indicate that stress has different effects on hippocampal and parahippocampal components of spatial memory. These findings suggest a possible mechanism by which cortisol responses serve as an adaptive function during stress and trauma, which may inspire future studies in this area.

\section{Acknowledgements}

This study was supported in part by Grant 056-25-011 from the Netherlands Organization for Scientific Research (NWO) to Dr. Tom Smeets. We are especially thankful to Marta Girardelli for her help in collecting the data and to Ron Hellenbrand (Maastricht University) for implementing the contextual cueing paradigm. There is no conflict of interest in the present study for any of the authors. 


\section{References}

Abercrombie, H. C., Kalin, N. H., Thurow, M. E., Rosenkranz, M. A., \& Davidson, R. J. (2003). Cortisol variation in humans affects memory for emotionally laden and neutral information. Behavioral Neuroscience, 117, 505-516. doi: 10.1037/07357044.117.3.505

Bennett, I. J., Barnes, K. A., Howard, J. H., \& Howard, D. V. (2009). An abbreviated implicit spatial context learning task that yields greater learning. Behavior Research Methods, 41, 391-395. doi: 10.3758/brm.41.2.391

Bisby, J. A., King, J. A., Brewin, C. R., Burgess, N., \& Curran, H. V. (2010). Acute effects of alcohol on intrusive memory development and viewpoint dependence in spatial memory support a dual representation model. Biological Psychiatry, 68, 280-286.

Brewin, C. R., Gregory, J. D., Lipton, M., \& Burgess, N. (2010). Intrusive images in psychological disorders: Characteristics, neural mechanisms, and treatment implications. Psychological Review, 117, 210-232. doi: 10.1037/a0018113

Chun, M. M., \& Jian, Y. H. (1998). Contextual cueing: Implicit learning and memory of visual context guides spatial attention. Cognitive Psychology, 36, 28-71.

Chun, M. M., \& Jiang, Y. H. (2003). Implicit, long-term spatial contextual memory. Journal of Experimental Psychology-Learning Memory and Cognition, 29, 224-234. doi: 10.1037/0278-7393.29.2.224

Chun, M. M., \& Phelps, E. A. (1999). Memory deficits for implicit contextual information in amnesic subjects with hippocampal damage. Nature Neuroscience, 2, 844-847.

de Kloet, E. R., Joels, M., \& Holsboer, F. (2005). Stress and the brain: From adaptation to disease. Nature Reviews Neuroscience, 6, 463-475. doi: 10.1038/nrn1683

de Quervain, D. J. F., Aerni, A., Schelling, G., \& Roozendaal, B. (2009). Glucocorticoids and the regulation of memory in health and disease. Frontiers in Neuroendocrinology, 30, 358-370. doi: 10.1016/j.yfrne.2009.03.002

de Quervain, D. J. F., \& Margraf, J. (2008). Glucocorticoids for the treatment of posttraumatic stress disorder and phobias: A novel therapeutic approach. European Journal of Pharmacology, 583, 365-371. doi: 10.1016/j.ejphar.2007.11.068

Domes, G., Heinrichs, M., Reichwald, U., \& Hautzinger, M. (2002). Hypothalamic-pituitaryadrenal axis reactivity to psychological stress and memory in middle-aged women: High responders exhibit enhanced declarative memory performance. Psychoneuroendocrinology, 27, 843-853. doi: 10.1016/S0306-4530(01)00085-3 
Fyhn, M., Hafting, T., Treves, A., Moser, M.-B., \& Moser, E. I. (2007). Hippocampal remapping and grid realignment in entorhinal cortex. Nature, 446, 190-194.

Gilbertson, M. W., Williston, S. K., Paulus, L. A., Lasko, N. B., Gurvits, T. V., Shenton, M. E., . . Orr, S. P. (2007). Configural cue performance in identical twins discordant for posttraumatic stress disorder: Theoretical implications for the role of hippocampal function. Biological Psychiatry, 62, 513-520. doi: 10.1016/j.biopsych.2006.12.023

Joels, M. (2011). Impact of glucocorticoids on brain function: Relevance for mood disorders. Psychoneuroendocrinology, 36, 406-414. doi: 10.1016/j.psyneuen.2010.03.004

Kirschbaum, C., Pirke, K. M., \& Hellhammer, D. H. (1993). The Trier Social Stress Test - A tool for investigating psychobiological stress responses in a laboratory setting. Neuropsychobiology, 28, 76-81. doi: 10.1159/000119004

Kudielka, B. M., Hellhammer, D. H., \& Wüst, S. (2009). Why do we respond so differently? Reviewing determinants of human salivary cortisol responses to challenge. Psychoneuroendocrinology, 34, 2-18.

Manns, J. R., \& Squire, L. R. (2001). Perceptual learning, awareness, and the hippocampus. Hippocampus, 11, 776-782. doi: 10.1002/hipo.1093

Meyer, T., Smeets, T., Giesbrecht, T., Quaedflieg, C. E. M., Girardelli, M., Mackay, G. N., \& Merckelbach, H. (2013). Individual differences in spatial configuration learning predict the occurrence of intrusive memories. Cognitive, Affective, \& Behavioral Neuroscience, 13, 186-196. doi: 10.3758/s13415-012-0123-9

Nicolson, N. A. (2008). Measurement of cortisol. In L. J. Luecken \& L. C. Gallo (Eds.), Handbook of physiological research methods in health psychology (pp. 37-74). Los Angeles: Sage.

Preston, A. R., \& Gabrieli, J. D. E. (2008). Dissociation between explicit memory and configural memory in the human medial temporal lobe. Cerebral Cortex, 18, 21922207. doi: 10.1093/cercor/bhm245

Pruessner, J. C., Kirschbaum, C., Meinlschmid, G., \& Hellhammer, D. H. (2003). Two formulas for computation of the area under the curve represent measures of total hormone concentration versus time-dependent change. Psychoneuroendocrinology, 28, 916-931. doi: 10.1016/s0306-4530(02)00108-7

Schwabe, L., Oitzl, M. S., Philippsen, C., Richter, S., Bohringer, A., Wippich, W., \& Schachinger, H. (2007). Stress modulates the use of spatial versus stimulus-response learning strategies in humans. Learning \& Memory, 14, 109-116. doi: $10.1101 / 1 \mathrm{~m} .435807$ 
Schwabe, L., Schachinger, H., de Kloet, E. R., \& Oitzl, M. S. (2010). Corticosteroids Operate as a Switch between Memory Systems. Journal of Cognitive Neuroscience, 22, 13621372. doi: 10.1162/jocn.2009.21278

Schwabe, L., Wolf, O. T., \& Oitzl, M. S. (2010). Memory formation under stress: Quantity and quality. Neuroscience and Biobehavioral Reviews, 34, 584-591. doi: 10.1016/j.neubiorev.2009.11.015

Smeets, T., Cornelisse, S., Quaedflieg, C. W. E. M., Meyer, T., Jelicic, M., \& Merckelbach, H. (2012). Introducing the Maastricht Acute Stress Test (MAST): A quick and noninvasive approach to elicit robust autonomic and glucocorticoid stress responses. Psychoneuroendocrinology, 37, 1998-2008. doi: 10.1016/j.psyneuen.2012.04.012

Smeets, T., Dziobek, I., \& Wolf, O. T. (2009). Social cognition under stress: Differential effects of stress-induced cortisol elevations in healthy young men and women. Hormones and Behavior, 55, 507-513. doi: 10.1016/j.yhbeh.2009.01.011

Taverniers, J., Smeets, T., Lo Bue, S., Syroit, J., Van Ruysseveldt, J., Pattyn, N., \& von Grumbkow, J. (2011). Visuo-spatial path learning, stress, and cortisol secretion following military cadets' first parachute jump: the effect of increasing task complexity. Cognitive Affective \& Behavioral Neuroscience, 11, 332-343. doi: 10.3758/s13415-011-0043-0

Taverniers, J., Taylor, M. K., \& Smeets, T. (2013). Delayed memory effects after intense stress in Special Forces candidates: Exploring path processes between cortisol secretion and memory recall. Stress, 16, 311-320. doi: 10.3109/10253890.2012.721824

Tollenaar, M. S., Elzinga, B. M., Spinhoven, P., \& Everaerd, W. (2008). Long-term outcomes of memory retrieval under stress. Behavioral Neuroscience, 122, 697-703. doi: 10.1037/0735-7044.122.3.697

Watson, D., Clark, L. A., \& Tellegen, A. (1988). Development and validation of brief measures of positive and negative affect: The PANAS scales. Journal of Personality and Social Psychology, 54, 1063-1070.

Wingenfeld, K., \& Wolf, O. T. (2011). HPA axis alterations in mental disorders: Impact on memory and its relevance for therapeutic interventions. CNS Neuroscience \& Therapeutics, 17, 714-722. doi: 10.1111/j.1755-5949.2010.00207.x

Wolf, O. T. (2008). The influence of stress hormones on emotional memory: Relevance for psychopathology. Acta Psychologica, 127, 513-531. doi: 10.1016/j.actpsy.2007.08.002 
Wolf, O. T., Schommer, N. C., Hellhammer, D. H., McEwen, B. S., \& Kirschbaum, C. (2001). The relationship between stress induced cortisol levels and memory differs between men and women. Psychoneuroendocrinology, 26, 711-720. doi: 10.1016/s0306-4530(01)00025-7 


\section{Chapter 4}

\section{The efficiency of reappraisal and expressive suppression in regulating everyday affective experiences}

Published as:

Meyer, T., Smeets, T., Giesbrecht, T., \& Merckelbach, H. (2012). The efficiency of reappraisal and expressive suppression in regulating everyday affective experiences. Psychiatry Research, 200, 964-969. 



\section{Abstract}

A widespread assumption in research and clinical practice is that cognitive reappraisal is a healthy and successful emotion regulation strategy, while expressive suppression is ineffective and has non-favourable consequences (e.g., decreased positive affect, higher physiological arousal). However, little is known about the consequences of reappraisal and expressive suppression for everyday affect. We investigated affective consequences of habitual reappraisal and expressive suppression in undergraduates $(\mathrm{N}=87)$, and sampled affect characteristics for $24 \mathrm{~h}$. Moreover, we quantified affective recovery from viewing an aversive video fragment. Habitual reappraisal was associated with lowered emotional arousal (but not valence), both in terms of diurnal affect levels and positive and negative responses to the emotional provocation task. This pattern contravenes the popular assumption that reappraisal has generally favourable consequences. Additionally, in contrast to the alleged nonfavourable consequences of habitual expressive suppression, the current study failed to find a relation between expressive suppression, diurnal affect levels and affective recovery. This suggests that the detrimental effects of expressive suppression are limited in duration. Collectively, our results emphasise that the everyday consequences of emotion regulation for affect merits systematic research, for instance by using more naturalistic and prolonged interventions.

\section{Keywords}

Emotion regulation; Day Reconstruction Method; provocation 


\section{Introduction}

Emotion serves as a guide for human behaviour and helps to prepare behavioural reactions quickly and in an automatic fashion (Most, Chun, Widders, \& Zald, 2005). However, environmental demands often determine whether an emotion should or should not be expressed or used as a guide for behaviour. To this end, humans are able to alter the strength and direction of their emotional responses (Bonanno, Papa, Lalande, Westphal, \& Coifman, 2004; Thompson, 1991) by employing emotion regulation strategies, reflecting a set of relatively stable, trait-like individual difference variables (Gross \& Thompson, 2007).

Researchers and clinicians have been intrigued by potential consequences of individual differences in emotion regulation. For instance, Berking et al. (2008) found that emotion regulation skills play a key role in emotional disorders, while Garnefski et al. (2002) argued that some emotion regulation strategies may be more adaptive than others. So far, the two emotion regulation strategies that have received most empirical scrutiny are cognitive reappraisal and expressive suppression. In the emotion regulation literature, these strategies have been differentiated on the basis of the emotion-generating system that is targeted (Koole, 2009) and where in the process of emotion-generation regulation occurs (Gross \& Thompson, 2007). Accordingly, reappraisal employs knowledge as emotion-generating system by changing cognitions about an emotional situation. Reappraisal occurs even before emotional response tendencies are fully generated. Expressive suppression, on the other hand, targets bodily responses as emotion-generating system. It diminishes the expression of response tendencies after they have been fully generated (Gross \& Thompson, 2007; Koole, 2009).

As to the acute consequences of these emotion regulation strategies, it has been demonstrated that reappraisal successfully reduces negative affect and/or increases positive affect (for a meta-analysis, see Augustine \& Hemenover, 2009). Expressive suppression seems to be ineffective. In contrast to reappraisal (but see Lam, Dickerson, Zoccola, \& Zaldivar, 2009), it may even produce undesirable effects such as decreased positive affect, increased physiological arousal, or memory impairments (e.g., Hofmann, Heering, Sawyer, \& Asnaani, 2009; for a review, see Gross, 2002). In addition to these transient effects, there are indications that reappraisal, but not expressive suppression, has favourable long-term consequences for individuals' well-being or interpersonal functioning (e.g., Haga, Kraft, \& Corby, 2009; John \& Gross, 2004). Regarding mental health, more frequent use of expressive suppression and less frequent use of reappraisal have been associated with depression, anxiety, and stress-related symptoms after trauma (Amstadter, 2008; Joormann \& Gotlib, 
2010; Moore, Zoellner, \& Mollenholt, 2008; but see Dunn, Billotti, Murphy, \& Dalgleish, 2009).

Surprisingly little is known about the impact of emotion regulation strategies on everyday affective experiences. Gross and John (2003) and Wang and co-workers (Wang, Shi, \& Li, 2009) used the Positive and Negative Affect Schedule (PANAS; Watson, Clark, \& Tellegen, 1988) as a measure of everyday affective experiences and found that habitual reappraisal was associated with generally higher positive and lower negative affect. In addition, experience sampling studies indicate that expressive suppression is associated with fewer daily positive events in individuals with elevated social anxiety (Kashdan \& Steger, 2006), and with higher daily stigma-related distress in minority groups (Hatzenbuehler, Nolen-Hoeksema, \& Dovidio, 2009). Also, Nezlek and Kuppens (2008) linked reappraisal to increased favourable affective consequences and expressive suppression to non-favourable consequences. A potential drawback of the above-mentioned studies is that they either conceptualised emotion regulation as a state variable (i.e., focusing on transient effects of emotion regulation within subjects) or used only one measurement of affect per day (e.g., mean or evening level per day). Thus, these studies did not establish whether stable individual differences in emotion regulation bear consequences for the dynamics of daily affective experiences.

To the best of our knowledge, only Kuppens, Oravecz, and Tuerlinckx (2010) provided a closer look into habitual emotion regulation and the dynamics of daily affect. In two experience sampling studies, these authors investigated the relationship between stable individual differences in emotion regulation and daily characteristics of two dimensions of affective experience (i.e., valence and arousal; Barrett \& Russell, 1999). Interestingly, habitual reappraisal was found not to be associated with the valence dimension of daily affect, yet it did relate to stronger regulation characteristics on the arousal dimension. Expressive suppression was related to higher daily arousal levels (Kuppens et al., 2010; Study 1) and to lower valence levels (Study 2).

The current study aimed to provide further insight into the consequences of habitual reappraisal and expressive suppression for everyday affective experiences by assessing affect characteristics both during a regular day and during an emotional provocation in a healthy undergraduate sample. More specifically, emotional valence and arousal were sampled across all distinct episodes of an entire day with the Day Reconstruction Method (DRM; Kahneman, Krueger, Schkade, Schwarz, \& Stone, 2004). The DRM allows for a quantification of diurnal affect characteristics, including variability in affect (i.e., temporal stability), independently for 
valence and arousal. In addition to linking reappraisal and expressive suppression to DRM valence and arousal dimensions, the current study also looked at how these emotion regulation strategies relate to the ability to restore positive and negative mood following an emotional provocation task.

Drawing from previous research (e.g., Kuppens et al., 2010; Nezlek \& Kuppens, 2008), we expected habitual reappraisal to correlate positively with mean levels and stability of DRM valence. We also anticipated reappraisal to correlate positively with stability (but not mean levels) of DRM arousal. As to the emotional provocation task, reappraisal was thought to be linked to an increased ability to restore mood levels during recovery (i.e., smaller increases in negative affect and smaller decreases in positive affect). ES was expected to correlate negatively with mean levels and stability of DRM valence as well as with stability of DRM arousal, but positively with mean levels of DRM arousal. Expressive suppression was also expected to be linked to an impaired ability to restore mood levels in recovering from the emotional provocation task.

\section{Methods}

\section{Participants}

Eighty-seven undergraduates (57 women) with a mean age of $21.5(S D=4.0$; range: 19 -45) voluntarily participated in the current study and in return received partial course credit. All participants were native Dutch speakers and provided written informed consent. The study was approved by the standing ethical committee of the Faculty of Psychology and Neuroscience, Maastricht University.

\section{Emotion regulation strategies}

A Dutch translation (Koole \& Jostmann, 2004) of the Emotion Regulation Questionnaire (ERQ; Gross \& John, 2003) was used to assess the habitual use of reappraisal and expressive suppression. The ERQ consists of a six-item reappraisal subscale $(\alpha=.85)$ and a four-item expressive suppression subscale $(\alpha=.79)$. Examples are "I control my emotions by changing the way I think about the situation I'm in" (reappraisal) and "I keep emotions to myself" (suppression). Participants are required to indicate whether they agree with each statement on a seven-point scale ranging from $1=$ strongly disagree to 7 = strongly agree. Reappraisal and expressive suppression scores are derived by averaging the item scores. In the present sample, mean reappraisal and suppression scores were $3.46(S D=1.01)$ and $4.91(S D=1.19)$. The 
subscales did not correlate with one another $(r=-0.05, n s)$. There was a significant gender difference in expressive suppression, with men relying on suppression less often than women by 2.58 points, $t(85)=2.47, p<0.05$. There were no gender differences in the use of reappraisal.

\section{Diurnal affect}

Diurnal affect characteristics were assessed with the Day Reconstruction Method (DRM; Kahneman et al., 2004). The DRM first required participants to write down all distinct episodes of the previous day that they could recollect. For each episode, participants indicated the beginning and end time and completed an Affect Grid. The Affect Grid (Russell, Weiss, \& Mendelsohn, 1989) reliably assesses emotional valence and arousal on a single-item scale presented as a two-dimensional space made up by nine horizontal points (ranging from unpleasant feelings on the left to pleasant feelings on the right) and nine vertical points (ranging from high arousal at the top to sleepiness at the bottom). Valence and arousal scores can range from -4 to +4 . Finally, participants indicated how typical the previous day was for that day of the week on a five-point scale $(1=$ much worse $;=$ much better $)$.

\section{Affective response to emotional provocation}

Stimuli. Participants were shown a 12 min video fragment from "The Silence of the Lambs" (Demme, 1991) that has been used in previous studies of our lab (e.g., Giesbrecht, Merckelbach, van Oorsouw, \& Simeon, 2010). Importantly, the selected fragment steadily increases in tension over time and elicits fearful emotions (Rottenberg, Ray, \& Gross, 2007). Robust mood effects in these prior studies attest to the usefulness of this video fragment for studying how mood may be influenced by individual differences in emotion regulation.

Affect change. Changes in positive and negative affect that outlasted the recovery from the emotional provocation were assessed by means of the state version of the Positive and Negative Affect Schedule (PANAS; Watson et al., 1988), administered immediately prior to the film (i.e., baseline) and immediately following a recovery period of five minutes (i.e., post-measure). The PANAS consists of two 10-item subscales measuring positive affect (PA; $\alpha \mathrm{s}>.89$ in both administrations) and negative affect (NA; $\alpha \mathrm{s}>.78)$. Items require participants to rate the extent to which they experienced certain emotions (e.g., PA: interested, NA: distressed $)$ on five-point scales $(1=$ very slightly or not at all; $5=$ very much). PA and NA change scores were determined by subtracting baseline from the post-measure scores. NA 
scores, but not PA scores, were log-transformed prior to calculating change scores to correct for a strong right-skewed distribution. To facilitate interpretation, the scores reported hereafter represent the untransformed means and standard deviations of the NA change scores.

Habituation and strength of the provocation. In order to address potential habituation effects to the film fragment, participants were asked whether they had previously seen the fragment. Also, participants were asked to indicate the maximum level of fear they had experienced during the film fragment on a 100mm Visual Analogue Scale (VAS; anchors: $0=$ not at all; $100=$ very much).

\section{Procedure}

Participants were seated individually at a desk in a soundproof room. First, the DRM was administered, each part being accompanied by extensive written and oral instructions. Next, participants completed the ERQ, followed by a short relaxation phase, and then the baseline PANAS was administered. An automated presentation was started on the computer screen, explaining to the participants that they were about to see an emotionally provoking film clip followed by $5 \mathrm{~min}$ of rest. Participants started the presentation of the video fragment themselves by pressing a response button, viewed the film clip, and were then instructed to sit quietly for another $5 \mathrm{~min}$. Importantly, no instructions concerning the regulation of emotions were given as we were interested in the habitual use of emotion regulation strategies. After the 5 min recovery period, the post-measure PANAS was administered followed by querying participants about their prior knowledge of the film fragment and administering the fear VAS.

\section{Statistical analysis}

In order to assess affect changes, baseline and post-measure PA and NA scores were compared by means of paired-samples $t$-tests. DRM data were summarised independently for valence and arousal by calculating mean levels across all reported episodes for each participant. Moreover, standard deviations across all episodes were used as indexes of variability, and mean squared successive differences (MSSDs) across all episodes were derived to estimate temporal stability (Ebner-Priemer, Eid, Kleindienst, Stabenow, \& Trull, 2009). Linear associations between the ERQ subscales and the DRM affect characteristics were tested by computing Pearson's correlation coefficients. Likewise, linear associations between the ERQ subscales and PA and NA change scores in response to the emotional provocation were tested. Also, Pearson correlations between the ERQ subscales and 
maximum fear VAS scores were calculated. $P$ 's $<.05$ were considered statistically significant; A priori hypotheses were evaluated with one-tailed tests. Explorative tests were two-tailed.

\section{Results}

\section{DRM diurnal affect}

Participants recalled on average 14.9 episodes of the previous day $(S D=4.4$; range: 6 25). Most participants indicated that the previous day was rather typical for that day of the week; only $11(12 \%)$ said that the previous day was "much worse" or "much better" than usual. A summary of the diurnal affect characteristics is provided in Table 4.1. Table 4.2 shows correlation coefficients between ERQ subscales and diurnal affect characteristics. As can be seen, there was a significant negative correlation between reappraisal and mean level of arousal $(r=-.31, p<.01)$. Reappraisal did not correlate significantly with arousal variability or temporal stability, nor with mean level, variability, and temporal stability of valence. Expressive suppression was unrelated to all DRM affect characteristics.

\begin{tabular}{llll}
\hline Diurnal affect characteristic & $M$ & $S D$ \\
\hline Valence & Mean level & 0.96 & 0.79 \\
& Variability & 1.82 & 0.49 \\
& Stability & 5.42 & 2.71 \\
Arousal & Mean level & 0.18 & 0.77 \\
& Variability & 2.02 & 0.43 \\
& Stability & 5.94 & 3.67 \\
\hline
\end{tabular}

Table 4.1. Raw descriptives of the Diurnal Affect Characteristics assessed with the Day Reconstruction Method $(N=85)$. Mean Level refers to the individual average scores, variability to individual standard deviations, and Stability to individual mean squared successive differences across all episodes of the assessed day; $S D=$ standard deviation. 


\begin{tabular}{lccc}
\hline & & \multicolumn{2}{c}{ ERQ } \\
\cline { 3 - 4 } Diurnal affect characteristic & Reappraisal & Suppression \\
\hline Valence & Mean level & -0.12 & 0.16 \\
& Variability & 0.06 & 0.00 \\
& Stability & 0.11 & 0.07 \\
Arousal & Mean level & $-0.31^{* * a}$ & 0.01 \\
& Variability & -0.07 & 0.11 \\
& Stability & 0.09 & 0.06 \\
\hline
\end{tabular}

Table 4.2. Pearson product-moment correlations between Emotion Regulation Questionnaire (ERQ) subscales and diurnal affect characteristics $(N=85)$. Mean level refers to the individual average scores, variability to individual standard deviations, and stability to individual mean squared successive differences across all episodes of the assessed day; ${ }^{\mathrm{a}} \mathrm{In}$ contrast to the other correlation analyses, no significant relationship was hypothesized between Reappraisal and Arousal Mean level, so that probability was tested using a two-tailed test instead of a one-tailed test; ** $p<0.01$.

\section{Emotional provocation}

Effects of the provocation. On average, participants reported a maximum fear score of 41.0 on the VAS ( $S D=28.0$; Range: 0 - 92). With respect to mood changes, average PA remained stable in the whole sample, $t(86)=0.57, n s, M_{\text {Difference }}=0.2(S D=3.18)$. Meanwhile, NA increased significantly from baseline to post-measure, $t(86)=2.18, p<.05$, $M_{\text {Difference }}=0.67(S D=3.03)$. About half of the sample $(n=45)$ indicated that they had previously seen the film fragment that was used for the emotional provocation. In order to assess whether PA and NA changes were influenced by familiarity with the film, 2 (Time: baseline, post-measure) by 2 (Group: seen, not seen) repeated measures ANOVAs were performed separately for PA and NA. No interaction between PA change and familiarity with the film fragment was found, $F(1,85)=0.26, n s$. However, a significant interaction effect for NA change emerged, $F(1,85)=5.37, p<.05$. Paired samples $t$-tests in the two subgroups showed that NA increased for participants who had never before seen the fragment $(n=42), t$ $(41)=2.93, p<.01, M_{\text {Difference }}=1.45(S D=3.27)$, while remaining stable in those who had seen the film fragment previously $(n=45), t(44)=.01, n s$. Familiarity with the film fragment did not affect the reported maximum fear level on the fear VAS, $t(85)=0.92, n s$. 
Impact of emotion regulation on affect change. Correlational analyses revealed statistically significant negative correlations between reappraisal and PA ( $r=-.24$, two-tailed $p<.05)$ as well as NA $(r=-.30$, one-tailed $p<.01)$ change scores. Suppression was statistically unrelated to the PANAS change scores. Maximum fear did not correlate with reappraisal, while a positive correlation with expressive suppression was found $(r=.20$, onetailed $p<.05)$.

Using two-tailed tests of significance, the correlation analyses were repeated separately for participants who had and those who had not seen the film before. The associations between reappraisal and PA change and NA change were carried by participants who recognised the film fragment (PA: $r=-.36, p<.05$; NA: $r=-.38, p<.05$ ) and were nonsignificant in participants who did not know the fragment (PA: $r=-.11, n s$; NA: $r=-.25, p=$ .11). A significant positive association between expressive suppression and maximum fear was found for participants who recognised the film fragment $(r=.32, p<.05)$, but not for those who did not know the film $(r=.06, n s)$.

\section{Discussion}

To date, only few studies have investigated the potential role of habitual emotion regulation in everyday affective experience. Using two independent paradigms, we investigated the link between aspects of everyday affective experience and the habitual use of cognitive reappraisal and expressive suppression. The main findings can be summarised as follows. First, no association was found between reappraisal and any of the diurnal characteristics of valence assessed with the DRM. There was, however, a moderate negative correlation between reappraisal and diurnal emotional arousal mean levels. Second, in the recovery from the emotional provocation, reappraisal predicted both lower change scores of NA and lower change scores of PA. However, associations between reappraisal and change in PA and NA were only significant in participants who were familiar with the fragment. Third, regarding the effect of the habitual use of ES, the results showed no association between expressive suppression and diurnal characteristics of valence, and no association between expressive suppression and diurnal characteristics of arousal on the DRM. Finally, no influence of habitual expressive suppression on PA or NA change scores after the emotional provocation was found, but suppression predicted higher maximum fear ratings. Post-hoc analyses showed that this association with higher maximum fear ratings was only evident for those participants who were familiar with the film fragment. 


\section{Consequences of habitual reappraisal}

The assumption that cognitive reappraisal is generally associated with favourable affective experiences was not supported unambiguously by our findings. That is, none of the DRM valence parameters was related to reappraisal. Instead, reappraisal was associated with lower mean DRM arousal, irrespective of participants' valence scores. In the emotional provocation task, on the other hand, habitual reappraisal was associated with a buffered increase in negative affect after recovery from the emotional provocation. This is consistent with previous studies that used emotional provocation tasks (Augustine \& Hemenover, 2009). These studies also found that reappraisal goes along with an enhanced ability to recover from negative affective provocations. However, in our study, reappraisal additionally predicted smaller increases in PA. Thus, reappraisal seemingly helps reducing NA at the cost of lowering the experience of positive emotion.

A possible explanation for this pattern lies in the factor structure of PA and NA. In a circumplex model of affect consisting of valence and arousal dimensions (Barrett \& Russell, 1999), the PA scale is regarded as aroused positive affect and the NA scale as aroused negative affect. Therefore, a simultaneous decrease of PA and NA, as we observed in those scoring high on reappraisal, can be interpreted as a decrease in affective arousal. Since reappraisal was also associated with lower diurnal levels of emotional arousal, we provide converging evidence from two paradigms for this interpretation. Interestingly, this interpretation is also in line with the Kuppens et al. study (2010), which showed a positive relationship between habitual reappraisal and arousal regulation. Our line of argumentation concurs with Kuppens et al.'s conclusion that the effects of reappraisal on affect might be limited to the arousal component of affective experience.

The post-hoc analyses for participants who were familiar with the film fragment and those who were not yielded some interesting additional findings. During the provocation, participants who recognised the film fragment had more contextual information at their disposal (e.g., they knew already how the scene would end), which may have facilitated changing the cognitive interpretation of the fragment. Alternatively, familiarity might have led to anticipation of fearful reactions, leading to a more pronounced engagement in emotion regulation processes. Both possibilities would dictate that the impact of cognitive emotion regulation strategies is more pronounced in the group that was familiar with the film. This was indeed the pattern that we found. Moreover, participants who recognised the film did not 
show an increase in NA due to the provocation, suggesting that this subgroup effectively regulated their emotions, which can in part be attributed to the habitual use of reappraisal.

\section{Consequences of habitual expressive suppression}

The current study found no support for the assumption that the habitual use of expressive suppression is associated with less favourable consequences for affective experiences. For instance, the present study failed to observe any relationship between habitual expressive suppression and daily affect characteristics. This contradicts some earlier studies that have related suppression to reduced general PA and increased general NA (e.g., Gross \& John, 2003; but see Wang et al., 2009). In the emotional provocation task, on the other hand, expressive suppression was only related to higher maximum fear levels. The absence of effects on PA and NA change scores might be accounted for by the fact that affect was measured after five minutes of recovery instead of directly after the provocation, tapping more pervasive changes rather than acute changes. That is, our findings suggest that expressive suppression does not have any notable consequences for affective experiences outlasting the recovery period. This might also explain the absence of associations with DRM affect characteristics. Additional support for this interpretation stems from Kuppens et al. (2010), who found an association between suppression and lowered valence in a four-days experience sampling study with 50 observations per day, but not in a two-weeks study with just 10 observations per day. The former, but not the latter study, might have captured valence fluctuations in response to minor daily stressors. Notable is also that Kuppens et al. found higher arousal levels associated with expressive suppression in the latter, but not in the former study, which they interpreted as evidence that habitual expressive suppression might influence weekly rather than daily patterns of arousal levels. In this sense, our study confirms the view that expressive suppression has no global influence on daily arousal characteristics.

The additional analyses for participants who were and who were not familiar with the video fragment suggest that expressive suppression had a negative short-term effect for some participants only. In particular, in participants with knowledge of the film, expressive suppression correlated with maximum subjective fear. Theoretically, a similar correlation would be expected in the other subgroup, as there is no obvious link between habitual expressive suppression and responding to familiar vs. unfamiliar material. Again, one possibility is that familiarity led to an anticipation of a fearful reaction, and this might have mediated the effect of expressive suppression on fearful responding. In any case, these 
findings suggest that the effects of expressive suppression are not universal but depend on contextual factors such as familiarity.

\section{Alternative interpretations}

About half of the participants were familiar with the film material used in the emotional provocation. This made it possible to perform post-hoc comparisons in which familiarity with the film fragment was treated as a dichotomous variable. Obviously, from a methodological point of view, such non-planned comparisons are far from ideal. For instance, the compared groups might differ from each other on other aspects that are relevant to emotion regulation (e.g., general seeking of emotional stimulation). Alternatively, the absence of NA change in those who were familiar with the film might indicate that these participants were habituated to the material, and might therefore have been less motivated to regulate emotions. However, in terms of maximum fear responding, the strength of the provocation was moderate in the whole sample and was not affected by familiarity with the film fragment. This suggests that the two subgroups did not differ in their levels of habituation or in the need to regulate emotions, although these possibilities cannot be ruled out entirely.

That aside, it seems fruitful to further investigate the role of contextual information in the effects of emotion regulation strategies. For instance, future studies might want to address this directly in a controlled manipulation of contextual information. Likewise, the role of fear anticipation as possible mediator of emotion regulation effects on affect might be a promising target for future research. In sum, our post-hoc results suggest that future research should try to understand the circumstances in which cognitive reappraisal has beneficial consequences. The consequences of expressive suppression might turn out to depend more on contextual information than previously thought.

A noteworthy observation was that women were found to rely on expressive suppression more often than men in the present sample. Prior findings suggest that women tend to use all emotion regulation strategies more often than men (e.g., Nolen-Hoeksema \& Aldao, 2011), with the exception of suppression, which was found more often in men than in women (Melka, Lancaster, Bryant, \& Rodriguez, 2011). It is unclear what the reason for this disparity of findings may be, and whether gender might be a mediating factor in the effects of emotion regulation on affective experiences. Future research to address these questions is warranted. 


\section{Limitations}

The following limitations deserve to be mentioned. To begin with, the sample only consisted of university students who might be exposed to emotional stressors to a lesser extent than other groups in the general population, and consisted of a relatively high-functioning group. Furthermore, although psychological complaints were not systematically assessed, such complaints may have been present and thus may have influenced the results of this study. Other limitations of our study concern the methods used for the assessment of affect characteristics. For instance, our conclusions are generally limited by the use of introspective reports of affect. Also, a possible critique is that the DRM assesses diurnal affect characteristics retrospectively in a single session. Nevertheless, the quality of DRM data has been shown to be comparable to experience sampling data in avoiding memory distortions (Kahneman et al., 2004). A limitation of our emotional provocation task is that we assessed peak emotional responding only retrospectively with a VAS, in order to avoid interference with the uninstructed emotion regulation process. Consequently, we are not able to thoroughly disentangle emotional responding from emotion regulation, i.e. the time course of affective responding. Although this was not the focus of our study, we cannot rule out that our results were influenced by individual differences in affective responding (e.g., Larsen \& Ketelaar, 1991). Likewise, we cannot rule out that the results may have been moderated by individual differences in the motivation to regulate emotions. Finally, it is also notable that we used a fear induction as emotional provocation, implying that our results may not generalise to other negative emotions.

\section{Conclusion}

A widely held assumption is that cognitive reappraisal has favourable consequences for affective experiences while expressive suppression has unfavourable consequences. While this assumption is reflected both in research and clinical approaches to emotion regulation (Berking et al., 2008; Garnefski et al., 2002), our findings do not yield unambiguous support for it. Whereas expressive suppression was largely unrelated to the measured affect characteristics, our results suggest that the effects of reappraisal are limited to the arousal component of affective experiences, which is in line with other findings (Kuppens et al., 2010). Future studies should further elucidate under which circumstances reappraisal leads to reduced emotional arousal, and whether such an arousal reduction can be regarded as favourable for everyday affective experiences. More generally, the present findings suggest 
that reappraisal and suppression might have less severe consequences for everyday life than previously thought and, at the same time, emphasise the importance of solidifying and further extending our knowledge about the consequences of cognitive reappraisal and expressive suppression for everyday affective experiences.

\section{Acknowledgement}

We are especially thankful to Liselore Dubois, Sumi el Houbba, Darya Moghimi, Daisy Ramackers, Marion Sumpf, and Helene Vos for their help in collecting the data. This study was supported by Grant 056-25-011 from the Netherlands Organization for Scientific Research (NWO) to Dr. Tom Smeets. 


\section{References}

Amstadter, A. (2008). Emotion regulation and anxiety disorders. Journal of Anxiety Disorders, 22, 211-221. doi: 10.1016/j.janxdis.2007.02.004

Augustine, A. A., \& Hemenover, S. H. (2009). On the relative effectiveness of affect regulation strategies: A meta-analysis. Cognition \& Emotion, 23, 1181-1220. doi: $10.1080 / 02699930802396556$

Barrett, L. F., \& Russell, A. A. (1999). The structure of current affect: Controversies and emerging consensus. Current Directions in Psychological Science, 8, 10-14.

Berking, M., Wupperman, P., Reichardt, A., Pejic, T., Dippel, A., \& Znoj, H. (2008). Emotion-regulation skills as a treatment target in psychotherapy. Behaviour Research and Therapy, 46, 1230-1237. doi: 10.1016/j.brat.2008.08.005

Bonanno, G. A., Papa, A., Lalande, K., Westphal, M., \& Coifman, K. (2004). The importance of being flexible - The ability to both enhance and suppress emotional expression predicts long-term adjustment. Psychological Science, 15, 482-487.

Demme, J. (Writer). (1991). The silence of the lambs: Orion Pictures.

Dunn, B. D., Billotti, D., Murphy, V., \& Dalgleish, T. (2009). The consequences of effortful emotion regulation when processing distressing material: A comparison of suppression and acceptance. Behaviour Research and Therapy, 47, 761-773. doi: 10.1016/j.brat.2009.05.007

Ebner-Priemer, U. W., Eid, M., Kleindienst, N., Stabenow, S., \& Trull, T. J. (2009). Analytic strategies for understanding affective (in)stability and other dynamic processes in psychopathology. Journal of Abnormal Psychology, 118, 195-202. doi: $10.1037 / \mathrm{a} 0014868$

Garnefski, N., Van den Kommer, T., Kraaij, V., Teerds, J., Legerstee, J., \& Onstein, E. (2002). The relationship between cognitive emotion regulation strategies and emotional problems: Comparison between a clinical and a non-clinical sample. European Journal of Personality, 16, 403-420. doi: 10.1002/per.458

Giesbrecht, T., Merckelbach, H., van Oorsouw, K., \& Simeon, D. (2010). Skin conductance and memory fragmentation after exposure to an emotional film clip in depersonalization disorder. Psychiatry Research, 177, 342-349. doi: 10.1016/j.psychres.2010.03.010

Gross, J. J. (2002). Emotion regulation: Affective, cognitive, and social consequences. Psychophysiology, 39, 281-291. doi: 10.1017/s0048577201393198 
Gross, J. J., \& John, O. P. (2003). Individual differences in two emotion regulation processes: Implications for affect, relationships, and well-being. Journal of Personality and Social Psychology, 85, 348-362. doi: 10.1037/0022-3514.85.2.348

Gross, J. J., \& Thompson, R. A. (2007). Emotion regulation: Conceptual foundations. In J. J. Gross (Ed.), Handbook of emotion regulation (pp. 3-24). New York: The Guilford Press.

Haga, S. M., Kraft, P., \& Corby, E. K. (2009). Emotion regulation: Antecedents and wellbeing outcomes of cognitive reappraisal and expressive suppression in cross-cultural samples. Journal of Happiness Studies, 10, 271-291. doi: 10.1007/s10902-007-9080-3

Hatzenbuehler, M. L., Nolen-Hoeksema, S., \& Dovidio, J. (2009). How Does Stigma "Get Under the Skin"?: The Mediating Role of Emotion Regulation. Psychological Science, 20, 1282-1289.

Hofmann, S. G., Heering, S., Sawyer, A. T., \& Asnaani, A. (2009). How to handle anxiety: The effects of reappraisal, acceptance, and suppression strategies on anxious arousal. Behaviour Research and Therapy, 47, 389-394. doi: 10.1016/j.brat.2009.02.010

John, O. P., \& Gross, J. J. (2004). Healthy and unhealthy emotion regulation: Personality processes, individual differences, and life span development. Journal of Personality, 72, 1301-1333. doi: 10.1111/j.1467-6494.2004.00298.x

Joormann, J., \& Gotlib, I. H. (2010). Emotion regulation in depression: Relation to cognitive inhibition. Cognition \& Emotion, 24, 281-298. doi: 10.1080/02699930903407948

Kahneman, D., Krueger, A. B., Schkade, D. A., Schwarz, N., \& Stone, A. A. (2004). A survey method for characterizing daily life experience: The day reconstruction method. Science, 306, 1776-1780.

Kashdan, T. B., \& Steger, M. F. (2006). Expanding the topography of social anxiety: An experience-sampling assessment of positive emotions, positive events, and emotion suppression. Psychological Science, 17, 120-128.

Koole, S. (2009). The psychology of emotion regulation: An integrative review. Cognition \& Emotion, 23, 4-41. doi: 10.1080/02699930802619031

Koole, S., \& Jostmann, N. B. (2004). Getting a grip on your feelings: Effects of action orientation and external demands on intuitive affect regulation. Journal of Personality and Social Psychology, 87, 974-990. doi: 10.1037/0022-3514.87.6.974

Kuppens, P., Oravecz, Z., \& Tuerlinckx, F. (2010). Feelings change: Accounting for individual differences in the temporal dynamics of affect. Journal of Personality and Social Psychology, 99, 1042-1060. doi: 10.1037/a0020962 
Lam, S., Dickerson, S. S., Zoccola, P. M., \& Zaldivar, F. (2009). Emotion regulation and cortisol reactivity to a social-evaluative speech task. Psychoneuroendocrinology, 34, 1355-1362. doi: 10.1016/j.psyneuen.2009.04.006

Larsen, R. J., \& Ketelaar, T. (1991). Personality and susceptibility to positive and negative emotional states. Journal of Personality and Social Psychology, 61, 132-140. doi: 10.1037//0022-3514.61.1.132

Melka, S. E., Lancaster, S. L., Bryant, A. R., \& Rodriguez, B. F. (2011). Confirmatory factor and measurement invariance analyses of the emotion regulation questionnaire. Journal of Clinical Psychology, 67, 1283-1293. doi: 10.1002/jclp.20836

Moore, S. A., Zoellner, L. A., \& Mollenholt, N. (2008). Are expressive suppression and cognitive reappraisal associated with stress-related symptoms? Behaviour Research and Therapy, 46, 993-1000. doi: 10.1016/j.brat.2008.05.001

Most, S. B., Chun, M. M., Widders, D. M., \& Zald, D. H. (2005). Attentional rubbernecking: Cognitive control and personality in emotion-induced blindness. Psychonomic Bulletin \& Review, 12, 654-661.

Nezlek, J. B., \& Kuppens, P. (2008). Regulating positive and negative emotions in daily life. Journal of Personality, 76, 561-579. doi: 10.1111/j.1467-6494.2008.00496.x

Nolen-Hoeksema, S., \& Aldao, A. (2011). Gender and age differences in emotion regulation strategies and their relationship to depressive symptoms. Personality and Individual Differences, 51, 704-708.

Rottenberg, J., Ray, R. R., \& Gross, J. J. (2007). Emotion elicitation using films. In J. A. Coan \& J. J. Allen (Eds.), The handbook of emotion elicitation and assessment (pp. 9-28). New York: Oxford University Press.

Russell, J. A., Weiss, A., \& Mendelsohn, G. A. (1989). Affect grid: A single-item scale of pleasure and arousal. Journal of Personality and Social Psychology, 57, 493-502.

Thompson, R. (1991). Emotional regulation and emotional development. Educational Psychology Review, 3, 269-307.

Wang, L., Shi, Z. B., \& Li, H. H. (2009). Neuroticism, extraversion, emotion regulation, negative affect and positive affect: The mediating roles of reappraisal and suppression. Social Behavior and Personality, 37, 193-194. doi: 10.2224/sbp.2009.37.2.193

Watson, D., Clark, L. A., \& Tellegen, A. (1988). Development and validation of brief measures of positive and negative affect: The PANAS scales. Journal of Personality $\begin{array}{llrl}\text { and Social } & \text { Psychology, } & \text { 54, }\end{array}$ 



\section{Chapter 5}

\section{Frontal EEG asymmetry as predictor of physiological responses to aversive memories}

\section{Published as:}

Meyer, T., Quaedflieg, C. W. E. M., Giesbrecht, T., Meijer, E., Abiad, S., \& Smeets, T. (2014). Frontal EEG asymmetry as predictor of physiological responses to aversive memories. Psychophysiology, 51, 853-865. 



\section{Abstract}

Evidence suggests that asymmetry in frontal electrical activity predicts responses to aversive experiences, such that higher left-sided activity might dampen responses to trauma reminders. We measured frontal asymmetry at rest and during viewing of a trauma film, and assessed startle responses to film-reminder images. To explore potential moderators, we compared two films (Study 1; $N=64$ ) and modulated reappraisal (Study 2; $N=72$ ). As expected, left frontal activation during film viewing predicted dampened responses in individuals who viewed a staged road accident. However, this effect tended to be reversed when a genocide documentary was used. In Study 2, all participants viewed the genocide film. Left frontal activity at rest again predicted higher startle responses, while reappraisal did not moderate the effects. Thus, the type of trauma film plays a crucial role in the effects of frontal asymmetry, which warrants further critical investigation.

\section{Key words}

Eye-blink startle paradigm; frontal alpha asymmetry; trauma film; aversive memories; posttraumatic stress disorder 


\section{Introduction}

A considerable proportion of the general population is exposed to highly aversive events, such as rape, abduction, severe accidents, or disasters, at least once in their lifetime (Ozer, Best, Lipsey, \& Weiss, 2003). Despite the deep emotional impact of such experiences, most individuals display successful psychological adjustment, or so-called resilience (Bonanno, 2004). However, there seem to be large individual differences in how well people can adjust to such experiences. That is, some victims develop debilitating symptoms following aversive experiences, such as highly distressing memory intrusions and exaggerated startle responses (Brewin, Gregory, Lipton, \& Burgess, 2010; Orr \& Roth, 2000). Thus, an intriguing question that arises is which individual characteristics may be responsible for resilience, and thus protect from such symptoms.

The present article focuses on so-called frontal asymmetry as a marker of resilience, and explores its potential role in responding to adversity by using an analogue trauma-film paradigm (Holmes \& Bourne, 2008) in healthy participants. Frontal asymmetry has been studied extensively in individual differences research on emotion and refers to the average difference in alpha-band activity between the left and right frontal areas across several minutes, measured with electroencephalography (EEG; Harmon-Jones, Gable, \& Peterson, 2010). Based on the assumption that alpha band power is inversely related to brain activity (Pfurtscheller, Stancak, \& Neuper, 1996), frontal asymmetry is widely assumed to reflect hemispheric differences in frontal brain idling or activity. Given its excellent internal consistency and reasonable test-retest reliability ranging from 0.5 to 0.6 across several weeks (Tomarken, Davidson, Wheeler, \& Kinney, 1992; Towers \& Allen, 2009), frontal asymmetry has been studied as a trait marker of individual differences. Most commonly, it is measured while participants are at rest, whereby it purportedly taps into stable individual differences of resting brain activity. More recently, several studies additionally measured frontal asymmetry during an emotional provocation (e.g., Goodman, Rietschel, Lo, Costanzo, \& Hatfield, 2013), following the argumentation that asymmetric activation during challenge more reliably taps into an individual's trait-like capability of emotional responding, compared with resting frontal asymmetry (Coan, Allen, \& McKnight, 2006).

Although its neuro-anatomical and psychological links with mental health and emotional responding are still poorly understood (Allen \& Kline, 2004; see also: Miller, Crocker, Spielberg, Infantolino, \& Heller, 2013), frontal asymmetry is thought to have broad implications for emotional experience and well-being. It has been described as an index of an 
individual's trait-like style of affective responding by reflecting the relative activity of lateralized brain systems subserving motivation (Davidson, 1998) or emotional valence (Heller, 1993). In line with this view, it has repeatedly been found to predict emotional and behavioural reactions to stressful situations, as well as the presence of psychopathology. For instance, more left-sided frontal activity at rest has been linked to superior affect and stress regulation (e.g., Koslov, Mendes, Pajtas, \& Pizzagalli, 2011; Tomarken, Davidson, Wheeler, \& Doss, 1992), lower levels of anxiety and depression (Thibodeau, Jorgensen, \& Kim, 2006), and lower risk of developing depression (Nusslock et al., 2011). Furthermore, left-sided frontal activation during a sad film, but not resting frontal asymmetry, was found to predict dampened effects of adverse life events on psychopathology in a prospective study (LopezDuran, Nusslock, George, \& Kovacs, 2012), which also emphasizes the merit of measuring frontal asymmetry in different conditions. Based on these observations, it is conceivable that more left-sided frontal activity at rest and during provocation can predict adaptive responding to aversive experiences.

Interestingly, Jackson et al. (2003) found a link between more left-sided frontal activity at rest and enhanced down-regulation of physiological responses to aversive stimuli. In particular, these authors used a sophisticated startle paradigm in which startle probes were administered both during and shortly after picture presentation. This enabled them to assess the chronometry of startle responding, which is thought to be driven by amygdala activation during confrontation with fear-associated stimuli (Davis, Walker, \& Lee, 1997). Therefore, in Jackson et al.'s experiment, startle responses after elicitor offset were of particular interest for emotion regulation. These startle responses index sustained affective processing, which is thought to be inversely related to the rapidity with which prefrontal structures down-regulate the amygdala to instantiate affective recovery (Goldsmith \& Davidson, 2004). Strikingly, Jackson et al. found that left-sided frontal activity at rest specifically predicted lower startle response magnitude after negative picture offset, indicating enhanced affective recovery. Partly corroborating these findings, Goodman et al. (2013) more recently showed that leftsided activity during a threat provocation, but not at rest, predicted attenuated startle responses. Further support for the involvement of frontal asymmetry in affect regulation stems from research showing that left-sided frontal activation has been observed during cognitive reappraisal (Parvaz, MacNamara, Goldstein, \& Hajcak, 2012). This emotion regulation strategy implies deliberately altering a situation's emotional impact by changing its interpretation (Gross \& John, 2003; see also Chapter 4), which is negatively associated with the severity of trauma-related symptoms (Boden et al., 2013) and is considered effective in 
reducing physiological responding (Gross \& John, 2003). Taken together, the available evidence shows that individual differences in frontal asymmetry at rest and during provocation can inform about physiological response regulation after aversive stimulation.

\section{Aims and outline of the present studies}

While the above-mentioned studies suggest an intimate involvement of frontal asymmetry in resilience, it is not clear whether it is also implicated in more specific processes relevant to the adjustment after aversive experiences. For instance, it has not yet been investigated whether this marker is related to affective responses to aversive memories. Memory-related affect regulation is crucial in the adjustment to adversity, since trauma victims often suffer from physiological hyper-arousal when confronted with stimuli that remind them of their traumatic experience (Orr \& Roth, 2000). Thus, our first aim was to test the idea that more left-sided frontal activity would predict enhanced emotion regulation when individuals are confronted with stimuli that remind them of aversive experiences. Following the findings of Jackson et al. (2003), we expected frontal asymmetry to predict downregulation of startle responses. Therefore, we conducted two independent studies in which we measured frontal asymmetry at rest in healthy participants, who were then subjected to an analogue trauma film paradigm (Holmes \& Bourne, 2008). While participants viewed a shocking film, asymmetric frontal activation with respect to baseline was also measured, and was included as an additional predictor of later physiological responses during memory activation. These changes in asymmetric frontal activation can be expected to be equally or perhaps even more predictive of later physiological responses than the resting asymmetry index. On the one hand, provocation-induced asymmetries might tap more reliably into individual differences, in line with the capability model (Coan et al., 2006). On the other hand, activation asymmetry during film viewing might mediate (mal)adaptive emotional responding leading to exaggerated startle (Coan \& Allen, 2004). Next, participants underwent an eye-blink startle paradigm (Jackson et al., 2003) to assess physiological responses during and after presentation of images that elicited memories of the previously seen aversive film, compared to unrelated images.

The second aim of the present studies was to assess how robust possible relations between frontal asymmetry and physiological responses to reminder stimuli are across different contexts. That is, just as other risk and resilience factors (e.g., McNally \& Robinaugh, 2011), frontal asymmetry may not display universal effects in responding to 
adversity, but could depend on additional situational factors. For instance, in Chapter 4 we found that habitual use of cognitive reappraisal predicted dampened affective responses to a fear-inducing film, but only in those individuals who were already familiar with the film (Meyer, Smeets, Giesbrecht, \& Merckelbach, 2012). Similarly, frontal asymmetry may help to reduce physiological responses to film-related memories only under favourable circumstances, e.g., when the content and context of the film permits or encourages reappraising it in a benign manner. Therefore, we tested our main hypotheses in two different types of trauma film (Study 1) and within a single trauma film that was followed by provision of positive and negative contextual information (Study 2).

In both studies, we hypothesized that frontal asymmetry, both at rest and during film viewing, would be predictive of physiological self-regulation in response to reminder images, irrespective of condition effects. In particular, more left-sided frontal activity was expected to predict dampened startle responses shortly after reminder picture offset. Additionally, we tested moderation by different conditions, speculating that the expected effects of frontal activity would be more pronounced when the trauma film can easily be interpreted in a benign manner (Study 1), and when participants receive positive rather than negative contextual information about a trauma film (Study 2).

\section{Study 1}

In this study, we first measured resting frontal asymmetry, and had participants then view one of two different films, while all of them afterwards viewed reminder images from both films in a startle paradigm. One film consisted of real-life footage from a documentary about the 1994 Rwandan genocide, whereas the other is a staged educational movie depicting a severe road accident. Parts of both films, as well as films with similar content (see Weidmann, Conradi, Grögera, Fehma, \& Fydrich, 2009), have been used frequently in trauma-film studies, where they successfully induced negative emotion, involuntary memories, and exaggerated startle responses upon presentation of reminder pictures (e.g., Holmes, James, Coode-Bate, \& Deeprose, 2009; Chapter 2). Thus, both films are well suited for our purpose. Their traumatic content clearly differs, which allows exploring the robustness of frontal asymmetry effects across trauma film types. In particular, the road accident film may be easier to interpret in a positive and meaningful way since it is staged (e.g., "No one was really harmed.") and has a more positive educational message (e.g., "Road accidents can be prevented."), compared to the genocide documentary. If such differences play a 
moderating role, one might expect more pronounced effects of frontal asymmetry on physiological responding to reminder stimuli in the road accident condition.

\section{Method}

\section{Participants}

Sixty-five healthy young undergraduates were recruited via advertisements at the university campus and received partial course credit or a small financial compensation in return for their participation. Individuals who responded to the advertisements were screened for eligibility using the following exclusion criteria: (1) recent psychological complaints, (2) drug or alcohol abuse or addiction, (3) medical conditions or medication that could affect physiological recordings, (4) high self-relevance of film-related stimuli including blood phobia, current pregnancy and a history of a severe accident, assault, or injury, and (5) dominant left-handedness. All participants had normal or corrected-to-normal vision, and in the latter case were asked to wear glasses instead of contact lenses to reduce blinking artefacts. One participant did not complete the experiment following technical failure and was excluded from all analyses. The remaining 64 (46 women) had a mean age of 20.6 years ( $S D$ $=2.3$; range: $18-29$ ). All participants provided written informed consent. The study was approved by the standing ethical committee of the Faculty of Psychology and Neuroscience, Maastricht University.

\section{Procedure}

Participants were invited for a single laboratory session that was scheduled in the afternoon to reduce time-of-day effects on frontal asymmetry (Velo, Stewart, Hasler, Towers, \& Allen, 2012). Beforehand, they were instructed by e-mail to refrain from drugs (including alcohol) for $24 \mathrm{~h}$, and from heavy physical activities, smoking and drinking coffee for $1 \mathrm{~h}$ prior to participation. Participants were seated in a sound-proof, electrically shielded testing room, in front of a 22-inch widescreen monitor (Philips, the Netherlands) at approximately 56 $\mathrm{cm}$ unrestrained viewing distance. They first completed questionnaires. Next and after preparing the skin with abrasive gel, electrodes for EMG, EOG, and EEG recordings were prepared. Participants were shown the raw recording signals to demonstrate common artefacts that occur with open and closed eyes due to body and eye movements, followed by measuring resting-state EEG. Subsequently, participants were randomly assigned to watch one of two 
trauma films and were instructed to imagine being a witness to these scenes. Before and after the trauma film, mood was assessed with questionnaires. Participants then underwent an eyeblink startle paradigm with the instruction to watch a series of pictures, some of which would be film-related, attentively (i.e., without looking away or closing the eyes, and in such a way that they would know exactly what was shown in the picture). At the end of the session, the electrodes were removed and participants received financial or course credit compensation for their efforts and were fully debriefed by e-mail. Figure 5.1 displays the timeline of this study.

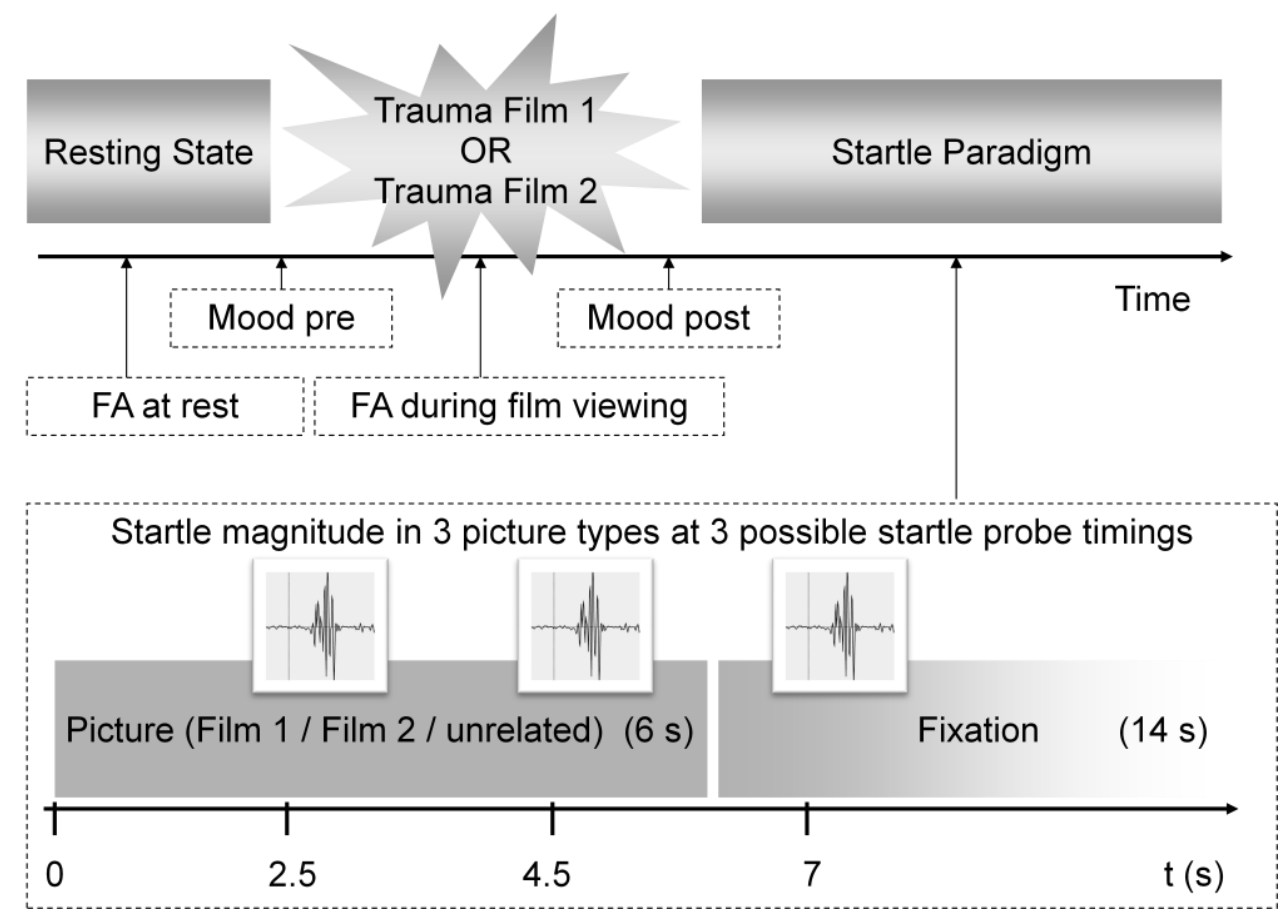

Figure 5.1. Timeline of study 1. Above the timeline, the order in which the tasks were administered is displayed. Below the timeline, the essential measures used in the analyses, as well as the general trial procedure of the eye-blink startle paradigm, are summarized. FA = frontal alpha asymmetry.

\section{Materials and tasks}

Questionnaires. The state version of the Positive and Negative Affect Schedule (PANAS; Watson, Clark, \& Tellegen, 1988) was administered to measure pre- and post-film positive affect $(\alpha \mathrm{s}>$.78) and negative affect $(\alpha \mathrm{s}>$.76). Additionally, Visual Analogue Scales (VAS) were used to measure changes in the current degree of feeling fearful, shocked, sad, 
and angry, ranging from $0=$ not at all to $100=$ very much. Furthermore, we included questionnaires at baseline and concerning dissociative responses to film viewing that are not addressed here. All questionnaires were computer-administered.

Trauma films. Two trauma film fragments, each lasting approximately $14 \mathrm{~min}$, were used. The first is a compilation of a documentary about the 1994 Rwandan genocide (Ghosts of Rwanda; Artwork PBS, 2004), consisting of real-life footage of death and mutilation with voice-over outlining background and extent of the events, next to interviews with affected individuals. The second film is a compilation of a road education movie co-produced by Gwent Police (http://www.gwent.police.uk), depicting a severe road accident, including the events leading up to it and its aftermath. In short, three young girls, one of whom is pregnant, become involved in an accident after arguing about a text message that the driver wants to send while driving. The accident causes severe injuries and even fatalities, and is followed by a dramatic rescue operation, all of which is shown in full detail.

Resting EEG recording. Similar to the procedure of Tomarken, Davidson, Wheeler, and Kinney (1992), the resting-state task consisted of eight 1 min blocks, half with eyes open and half with eyes closed. The beginning of each block was signalled by a single tone, and the end of each block was signalled by a double tone. Between blocks, instructions to close or open the eyes in the next block appeared for $20 \mathrm{~s}$. The order of conditions was randomized for each participant, whereby each condition was repeated no more than twice in a row.

Eye-Blink Startle Paradigm. Ninety different images served as stimuli in the startle paradigm (Jackson et al., 2003; Meyer et al., 2013). Pictures belonged to one of three categories: 30 reminder pictures from each of the two trauma film fragments, next to 30 neutral control (medium valence, low arousal ratings) pictures from the International Affective Picture System (IAPS; Lang, Bradley, \& Cuthbert, 2005). Reminder pictures were screen captures from the two fragments that contained no graphically disturbing details (i.e., the reminder pictures were chosen to be as neutral as possible). Since the trauma films differed strongly from each other visually and conceptually, reminder images of the unseen film were very unlikely to activate memories of the seen film.

As displayed in Figure 5.1, lower panel, each trial consisted of a picture that was shown for $6 \mathrm{~s}$, followed by and inter-trial-interval of $14 \mathrm{~s}$. This picture was accompanied by a $50 \mathrm{~ms}$ white noise startle probe (near-instantaneous rise time) presented via headphones. Intensity of the probes was set at $90 \mathrm{~dB}$. Probe onset occurred at $2.5 \mathrm{~s}, 4.5 \mathrm{~s}$, or $7 \mathrm{~s}$ relative to stimulus onset, with each timing occurring in 30 trials. The trial sequence was randomized with no more than three consecutive trials having the same probe onset or picture category. To reduce 
the predictability of startle probes, 6 trials without startle probe were inserted into the trial sequence (two from each picture category). Three pauses were inserted to avoid fatigue.

\section{Physiological recordings}

Following published guidelines (Keil et al., 2013; Pivik et al., 1993), EEG was measured with $\mathrm{Ag} / \mathrm{AgCl}$ electrodes in an electrode cap positioned according to the international 10-20 system (American Electroencephalographic Society, 1994), including the channels FZ, F3, F4, F7, F8, FC3, FC4, CZ, P3, P4, P7, P8, and A2, and using a BrainAmp EEG amplifier (Brainproducts $\mathrm{GmbH}$, Germany). Signals were sampled continuously at 1000 $\mathrm{Hz}$, referenced online to the left mastoid (A1), band-pass filtered $(0.1-35 \mathrm{~Hz})$ and stored. An electrode at AFz served as signal ground. EOG electrodes were applied above and below the right eye for vertical, and at the outer canthus of each eye for horizontal eye movement recording. Simultaneously, following the guidelines by Blumenthal et al. (2005), EMG from the orbicularis oculi muscle was continuously sampled at $1000 \mathrm{~Hz}(0.1-499 \mathrm{~Hz}$ band-pass filtered), using two $\mathrm{Ag} / \mathrm{AgCl}$ electrodes below the participant's left eye. All electrode impedances were kept below $5 \mathrm{kOhms}$ with homologous scalp electrodes being within 1 kOhms of each other.

\section{Data reduction}

EEG asymmetry scores. Consistent with data reduction procedures in previous frontal asymmetry studies (for a review, see Allen, Coan, \& Nazarian, 2004), EEG data were rereferenced offline to the average of A1 and A2 and band-pass filtered from 1 to $30 \mathrm{~Hz}$. To derive resting-state frontal asymmetry scores, each 1 min block was divided in 2 sec epochs (75\% overlap). Epochs were defined as artefact-contaminated (e.g., by eye-movement or muscle activity) and removed when vertical EOG or EEG activity exceeded thresholds of \pm 200 and $\pm 75 \mu \mathrm{V}$, respectively. Data from one participant (road-accident condition) were excluded because only $8 \%$ of epochs in the eyes-open condition were artefact-free. For all other participants, on average $78.9 \%$ (range $=43-100 ; S D=17.2$ ) of eyes-open and $96.7 \%$ (range $=60-100 ; S D=7.4$ ) of eyes-closed epochs were artefact-free. Power density for each retained epoch was derived by fast-Fourier transformation (FFT) using a 100\% Hanning window. Average power densities were calculated separately for the eyes-open and eyesclosed conditions and weighted for the number of artefact-free epochs. Log-transformed 
alpha-power density $(8-13 \mathrm{~Hz})$ values were used to calculate asymmetry scores (i.e., $\ln [$ right $]-\ln [$ left $])$, that were then averaged across eyes-open and eyes-closed conditions.

For our analyses, we concentrate on alpha power asymmetry in the widely used midfrontal leads (F4, F3), and on the midline fronto-central leads (FC4, FC3), for which sites Jackson and colleagues (2003) demonstrated a relationship with startle responding. Since we did not have separate hypotheses for frontal and fronto-central asymmetries and in order to reduce the number of statistical analyses (and consequentially the probability of Type 1 error), we used a composite frontal asymmetry index (mean of $\ln [\mathrm{F} 4], \ln [\mathrm{FC} 4]-$ mean of $\ln [\mathrm{F} 3]$, $\ln [\mathrm{FC} 3]$ ). For one participant (road-accident condition), frontal asymmetry could not be determined due to corrupted recordings in one of the frontal channels.

In order to obtain activation asymmetry scores, frontal asymmetry during film viewing was computed similarly to resting state asymmetry. Continuous recordings (approximately 14 min) were divided in $2 \mathrm{sec}$ epochs (75\% overlap) to derive frontal asymmetry scores at the fronto-central leads. Data from 5 participants (3 from the road-accident condition) for whom less than $25 \%$ artifact-free epochs could be retained were excluded from these analyses. For all other participants, on average $66.2 \%$ (range $=25-98 ; S D=19.8)$ of all epochs $(N=1658)$ were artefact-free. This percentage did not differ between the film conditions, $t(57)=.72, p=$ .47 . Activation asymmetry scores were derived by subtracting resting state asymmetry from frontal asymmetry scores during film viewing. Finally, data were available from 62 participants (30 in the road-accident condition) for analyses of resting frontal asymmetry, and from 58 participants (28 in the road-accident condition) for analyses involving frontal activation asymmetry.

Startle responses. Calculation of startle responses followed the recommendations by Blumenthal et al. (2005). EMG signals were converted into a bipolar channel, $28 \mathrm{~Hz}$ highpass filtered, rectified, and smoothed using a $40 \mathrm{~Hz}$ low-pass filter. For each trial, EMG signals were extracted from $-50 \mathrm{~ms}$ to $250 \mathrm{~ms}$ relative to startle probe onset. The period from $50 \mathrm{~ms}$ to probe onset was used for baseline correction and was inspected semi-automatically for artefacts (e.g., EMG or vertical EOG exceeding a threshold of $\pm 20 \mu \mathrm{V}$ or $\pm 200 \mu \mathrm{V}$, respectively, or reflex onset before probe onset). By means of the EMG onset search algorithm implemented in Vision Analyzer 2.0 (Brain Vision), reflex onset was extracted automatically between 20 and $120 \mathrm{~ms}$ after probe onset (criterion: $4 S D$ with respect to baseline), as well as the peak value following onset, followed by a visual check of the onset search. This yielded a startle magnitude per trial (peak minus onset values). Trials without eye-blink response were rated as zero. Data from 3 participants ( 1 in the road-accident 
condition) had to be excluded because onsets and peaks could not be determined in a majority of responses. Startle magnitudes deviating more than $3 S D$ from the individual's mean were replaced by the mean $\pm 3 S D$ to treat extreme values (i.e., Winsorizing; see Rivest, 1994). Magnitudes were then square root-transformed and averaged within subjects for each stimulus category and startle probe time, respectively. Startle potentiation scores (i.e., mean magnitude on film trials minus mean magnitude on neutral trials) were calculated per probe time, separately for seen film and not-seen film probes.

\section{Statistical analysis}

Single extreme scores in the distributions of EEG asymmetry and startle potentiation were replaced so that their deviance from the sample mean equalled 2.5 times the sample $S D$ (Rivest, 1994). Next, in order to reduce their right-skewed distributions, PANAS-negative affect and negative emotion VAS scores were log-transformed prior to the analyses. For better readability, uncorrected descriptive statistics are reported. Effects of the interventions on frontal asymmetry, current emotion, and startle potentiation were addressed using repeated measures ANOVAs and $t$-tests. The main hypotheses were addressed using multiple linear regression and correlation analyses, with seen-film startle potentiation after picture offset (i.e., at the $7 \mathrm{~s}$ probe timing) scores as dependent, and frontal asymmetry scores as independent variables. To assess possible interactions with film condition, this between-subjects factor was entered in all analyses. In the regression models, film condition (dummy variable) and interaction terms of frontal asymmetry by film condition (i.e., $z$-transformed asymmetry scores $\mathrm{x}$ film condition dummy) were thus entered as predictors. To assess the specificity of effects, we also explored whether frontal asymmetry also predicted startle potentiation during picture viewing (i.e., at the 2.5 and $4.5 \mathrm{~s}$ timing) and whether parietal alpha asymmetry (at P4/P3) predicted startle potentiation after picture offset. When sphericity assumptions for ANOVAs were violated, Greenhouse-Geisser corrected $p$-values, along with the respective epsilon and uncorrected degrees of freedom are reported. Alpha was set at .05 (two-tailed) for all analyses.

In our main analyses, resting frontal asymmetry is modelled as a moderator between affective responding to film viewing and startle potentiation (see Figure 5.2, panel A). Frontal activation asymmetry, however, may both moderate and mediate the effects of film viewing on startle responses. In order to assess the plausibility of a mediation model (see panel B), we tested whether film viewing systematically changed asymmetry scores. Furthermore, we 
explored whether this change corresponds to the subjective intensity of responding to the film. Finally, in order to elucidate the interdependence of trait and state frontal asymmetry in the current study, we report correlations between these indices.

\section{Results}

\section{Resting and activation asymmetries}

A repeated measures ANOVA showed no difference between resting and film-viewing frontal asymmetries, and no effects involving film condition, all $p \mathrm{~s}>.17$. Further at odds with a mediation model (Figure 2, panel B), frontal activation asymmetry did not correlate with any PANAS or VAS change score, all $r$ s $(58)<.20, p>.13$, which was not moderated by the type of film that had been seen, all interaction $p \mathrm{~s}>$.11. In line with previous studies (e.g., Goodman et al., 2013), resting frontal asymmetry scores $(M=0.06, S D=0.12)$ correlated positively with asymmetry during film viewing $(M=0.05, S D=0.14), r=.85, p<.001$, but no correlation with frontal activation asymmetries emerged $(M=0.0, S D=0.08), r=.04, p=$ .75. Figure 5.3 displays the raw power density across frequencies from which alpha asymmetry scores were derived, as a function of condition. 


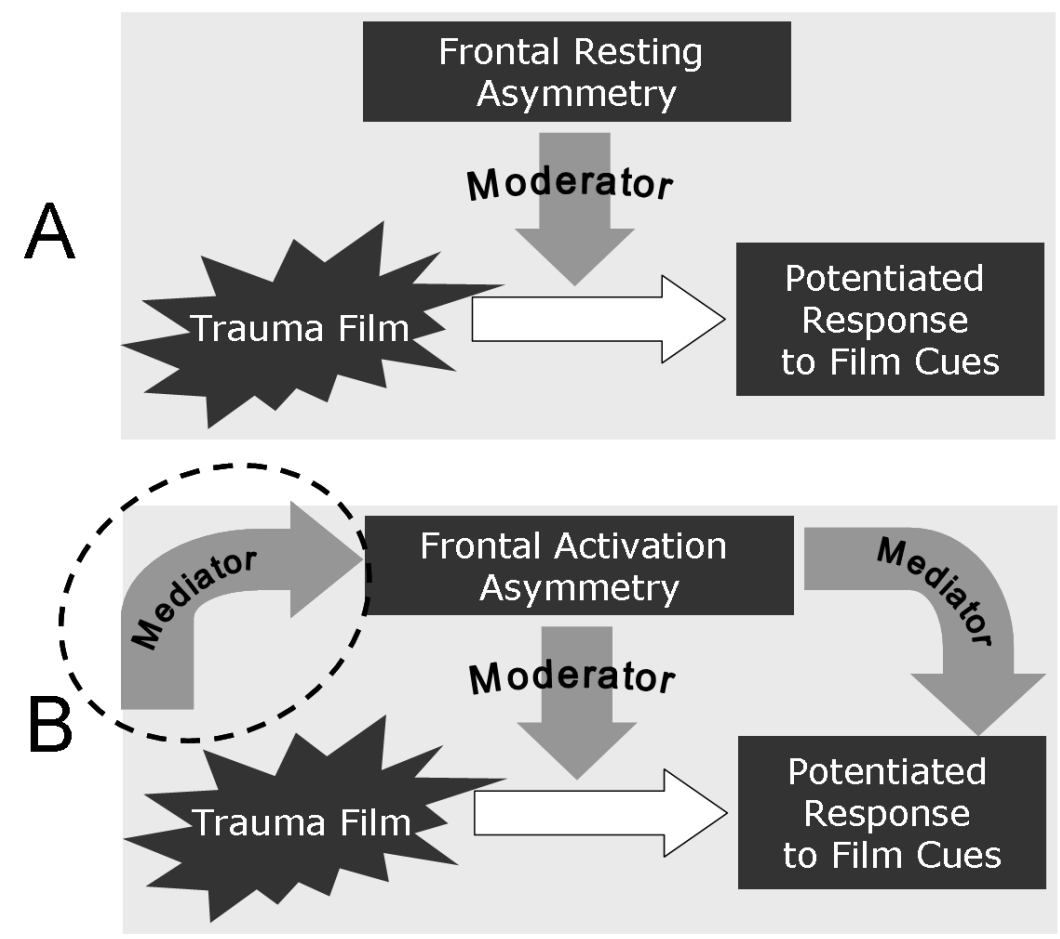

Figure 5.2. Theoretical models linking frontal asymmetries and startle responses. Panel A depicts the potential involvement of resting frontal asymmetry as a moderator of potentiated startle responses following film viewing. Panel B shows the potential involvement of activation asymmetry during film viewing. Similarly to resting asymmetry, it might act as a moderator, but could as well reflect (mal)adaptive responses that mediate later effects on startle. All models predict statistical associations between asymmetry and physiological responses. However, only the mediation model additionally predicts an association between trauma film responding and activation asymmetry, as highlighted by the dashed circle. 


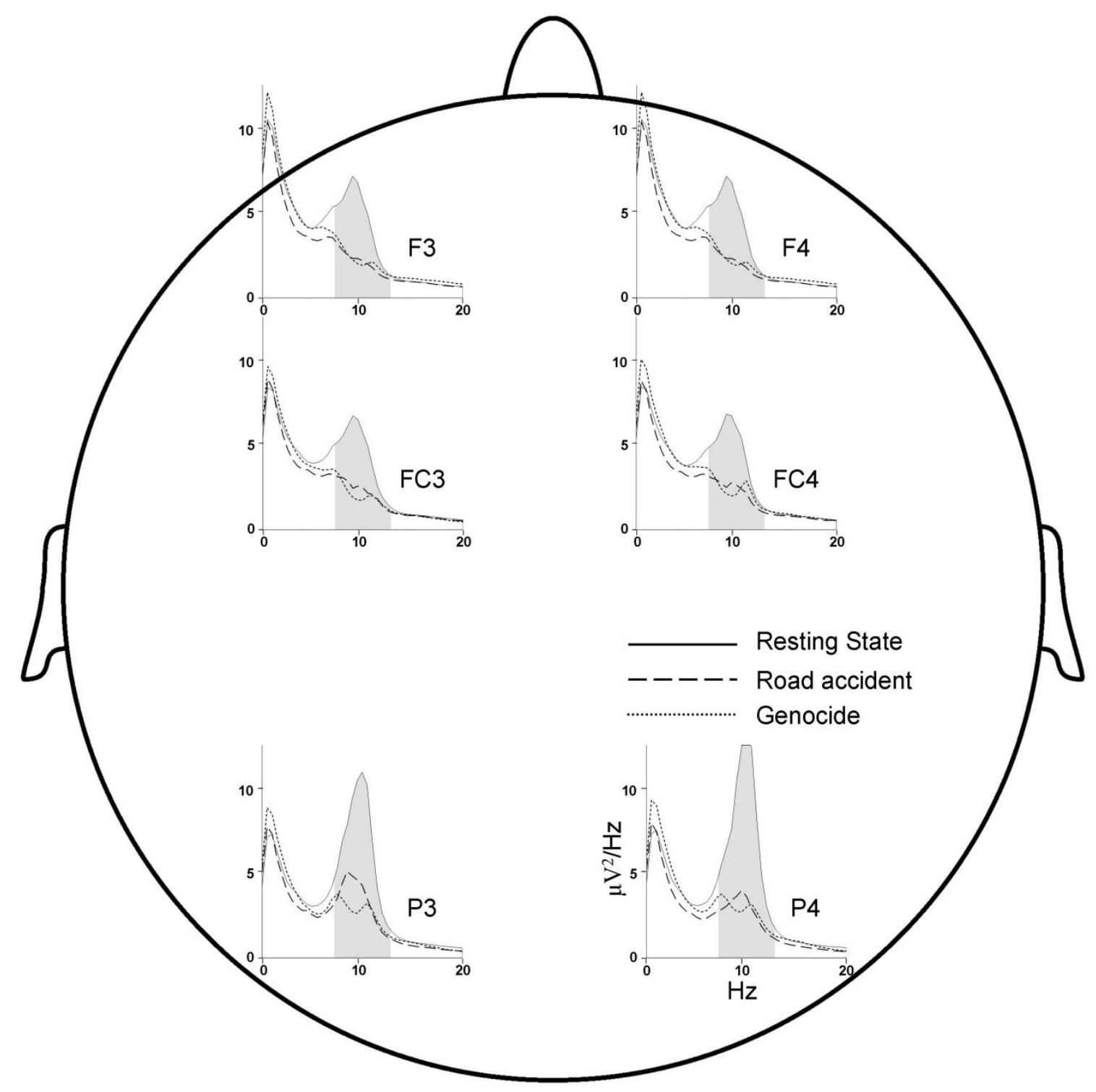

Figure 5.3. Topographical display of power density as a function of condition. The lines represent average power densities across frequencies at rest (solid lines; collapsed across conditions) and during film viewing (dashed lines), separately for the road accident $(n=28)$ and during the genocide film condition $(n=30)$. The grey area marks the alpha band, in which power densities were averaged to derive alpha asymmetry scores. 


\section{Emotional responses}

The two film conditions did not differ at baseline on any PANAS or emotion VAS subscale, all $t \mathrm{~s}(62)<1.2$, ps $>.25$. For PA, a 2 (Time: pre, post) by 2 (Film Condition: genocide, road-accident) repeated measures ANOVA revealed a significant main effect of Time, $F(1,62)=5.3, p=.025, \eta 2 p=.08$, with positive affect decreasing by 1.5 points $(S D=$ 5.3 ) in response to film viewing, in the absence of main or interaction effects involving Film Condition, $F \mathrm{~s}<1, p \mathrm{~s}>.82$. For negative affect, a significant Time effect was revealed, $F$ (1, $62)=80.5, p<.001, \eta 2 p=.57$, but also a significant interaction with Film Condition emerged, $F(1,62)=20.7, p<.001, \eta 2 p=.25$. An independent samples $t$-tests on negative affect change scores showed a stronger increase in the genocide condition $\left(\right.$ Mean $_{\text {change }}=8.8$, $S D=5.2)$ than in the road-accident condition $\left(\right.$ Mean $\left._{\text {change }}=2.3, S D=4.8\right), t(62)=4.5, p<$ .001 .

For the negative emotion VAS items, a 4 (Emotion) by 2 (Time) by 2 (Film Condition) repeated measures ANOVA revealed a significant three-way interaction, $F(3,186)=5.7$, Epsilon $=.79, p=.002, \eta 2 p=.08$. Paired samples t-tests in the two film conditions showed no significant increase in fearfulness for both the genocide and the road-accident condition, $p s>$ .16. The other three emotions (shocked, sad, angry) increased significantly in both conditions, with all $t \mathrm{~s}(31)>3.4$, all $p \mathrm{~s}<.01$ (see Figure 5.4). Independent samples $t$-tests showed that the increases in feeling shocked and angry were larger in the genocide condition (Mean change $_{\text {che }}$ being 50.8 and 42.1 , respectively, $S D=32.5,32.5)$ compared with the road-accident condition (Mean $_{\text {change }}$ being 25.3, 8.8, respectively, $\left.S D=27.2,21.6\right)$, $t \mathrm{~s}(62)>2.6, p<.02$. No differences were evident for anxiety and sadness change, $t \mathrm{~s}(62)<1.3, p \mathrm{~s}>.20$.

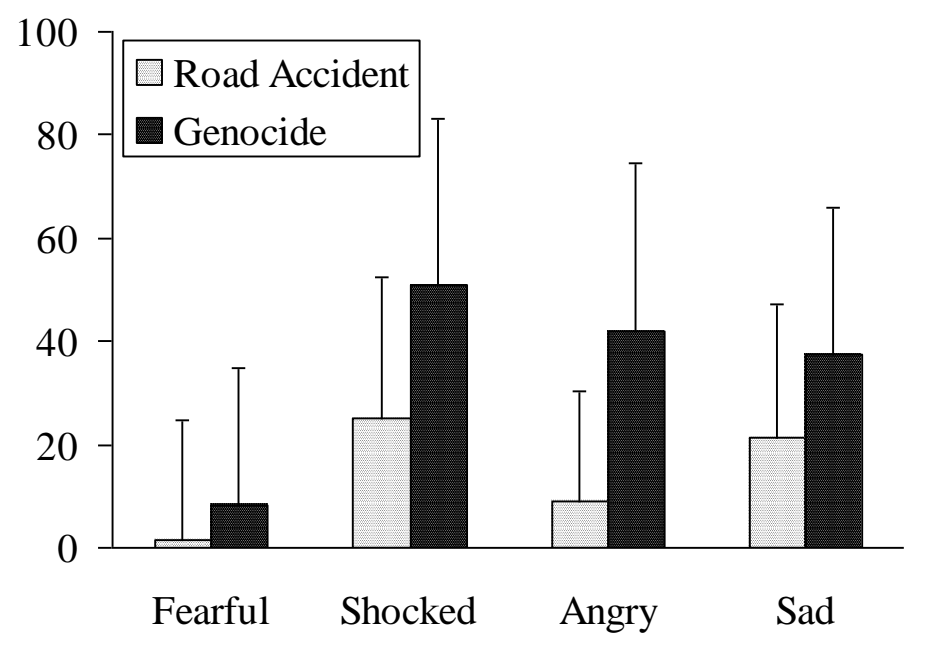

Figure 5.4. Specific negative emotion changes in response to film viewing on 100 mm VAS. $N=32$ in each film condition. Error bars indicate standard deviations. 


\section{Condition effects on time course of startle potentiation}

A 2 (Category: seen, unseen) by 3 (Timing) by 2 (Film Condition) repeated measures ANOVA on startle potentiation scores revealed no significant main or interaction effects of Category, Timing, or Film Condition (all $p s>.25$ ), except for a significant Category by Film Condition interaction, $F(1,59)=5.3, p=.03, \eta 2 p=.08$. Follow-up repeated measures ANOVAs per Film Condition showed a main effect of Category in the genocide film condition, $F(1,29)=4.2, p=.05, \eta 2 p=.13$, with seen-film potentiation scores being higher than unseen-film scores, which effect was not evidenced in the road accident condition, $F$ (1, 30) $=1.5, p=.24, \eta 2 p=.05$.

\section{Frontal asymmetry effects on startle potentiation ${ }^{1}$}

We first ran regression models to explore interactive effects of frontal asymmetry and film condition on startle potentiation after seen-film picture offset (i.e., at the $7 \mathrm{~s}$ probe timing, measuring emotion regulation). For resting frontal asymmetry $(d f=3,55)$, the interaction term was not significant, $p=.13$. In the entire sample, resting frontal asymmetry did not correlate significantly with startle potentiation after reminder picture offset $(r$ [59] = $.18, p=.17)$. In the models using frontal activation asymmetry as a predictor $(d f=3,51)$, the interaction term was a significant predictor, $\beta=.54, t=3.1, p=.003$. Therefore, we performed correlation analyses separately for the two film conditions. In the road accident condition, left-sided frontal activation correlated significantly and negatively with startle potentiation, $r(27)=-.47, p=.014$. In contrast, in the genocide condition, a positive trend emerged, $r(28)=.32, p=.095$ (see Figure 5.5).

For comparison, parietal resting and activation asymmetries (sites P4/P3) did not display any interaction effects with film condition on seen-film startle potentiation, $p \mathrm{~s}>.40$, and there were also no significant correlations in the entire sample, $p s>.41$. To assess the specificity of frontal asymmetry effects on startle potentiation after picture offset, we also looked at interaction and main effects for startle probes during picture presentation. This revealed no additional interaction effects for resting frontal asymmetry and also no significant correlations in the entire sample, all $p \mathrm{~s}>.47$. However, for activation asymmetry, there was a significant

1 None of the PANAS and emotion VAS change scores correlated significantly with any of the startle potentiation scores for seen-film trials, all $r \mathrm{~s}<.22, p \mathrm{~s}>.09$. Therefore, we did not consider these variables as additional predictors in the following analyses. 
interaction with film condition at the $2.5 \mathrm{~s}$ probe, $\beta=.39, t=2.2, p=.033$. Although no significant correlations between frontal activation asymmetry and startle potentiation emerged in either of the film conditions, the pattern was similar to the $7 \mathrm{~s}$ probe timings, with negative correlation coefficients in the road accident condition, $r(27)=-.29, p=.14$, and positive coefficients in the genocide condition $r(28)=.30, p=.12$, which accounted for the significant interaction.

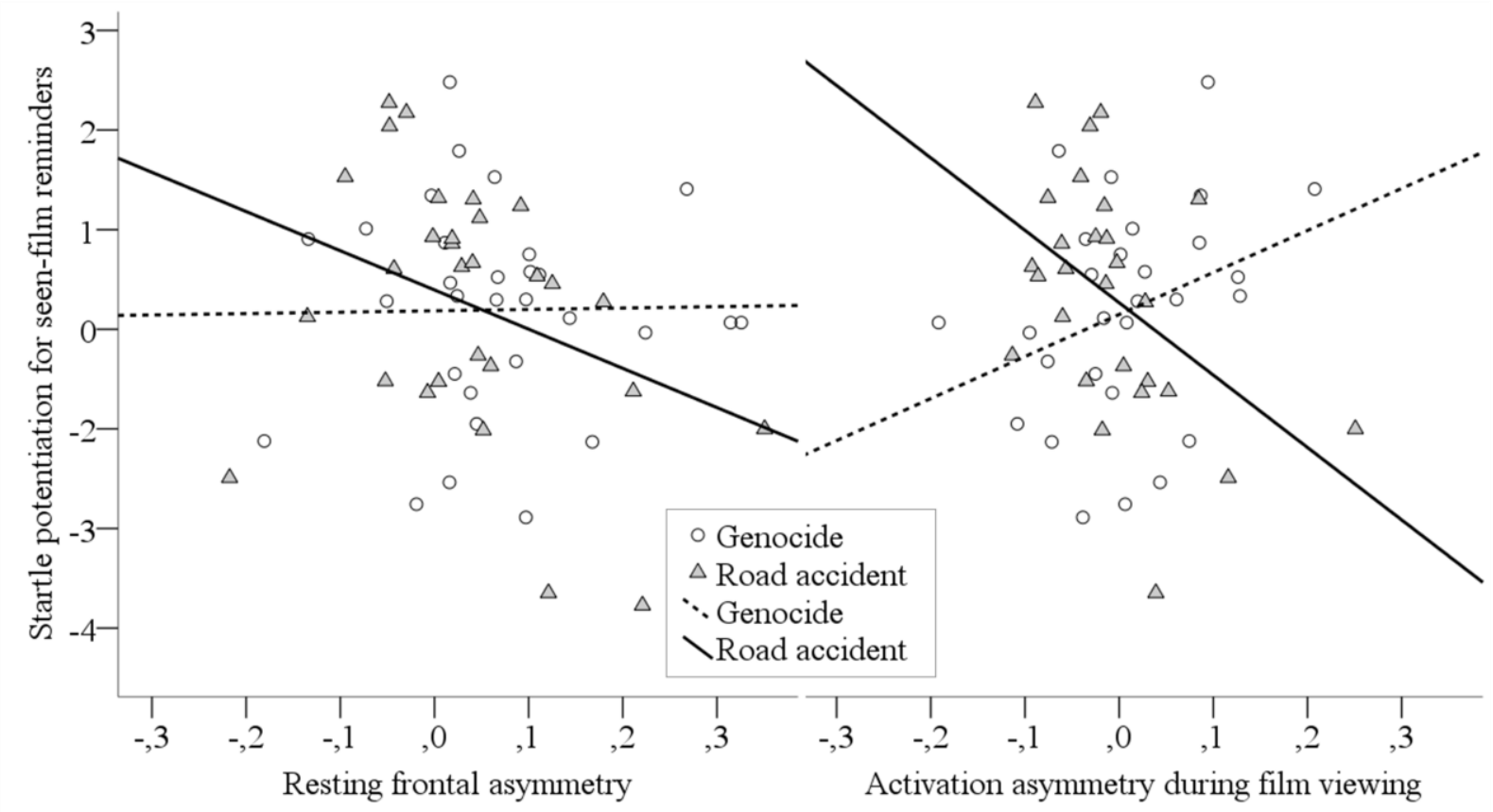

Figure 5.5. Scatter-plots depicting frontal asymmetry at rest (left panel; $N=59$ ) and activation asymmetry during film viewing (right panel; $N=55$ ) as predictors of startle potentiation at $7 \mathrm{~s}(1 \mathrm{~s}$ post-stimulus offset) for seen-film reminders. The interaction with film condition is significant for activation asymmetry, where a significant negative correlation emerged for the road accident condition only.

\section{Conclusion}

Resting frontal asymmetry was not predictive of physiological responding to traumafilm reminders in Study 1, while our results indicate that the effect of film-related activation asymmetry was moderated by film condition. Thus, our findings do not support the idea that frontal asymmetry predicts physiological responding to emotional memories in a straightforward manner. We found the predicted association between more left-sided frontal activation with enhanced physiological self-regulation in reminders (i.e., dampened startle after picture offset), but only in the road-accident condition. Meanwhile, a dissimilar effect 
was found in participants who saw the genocide film. Here, contrary to expectations, leftsided frontal activation not only appeared to have less dampening effects on startle, but we even observed a (non-significant) positive correlation with startle after picture offset. Thus, the two film conditions were apparently characterized by effects that clearly differed in size, and possibly also in direction. This suggests differences in emotion regulatory processes between the two film conditions. Speculatively, the road accident film may have been easier to reappraise in a benign manner, and to integrate with existing schemas, than the genocide film. An explanation for our findings might be that the effects of frontal asymmetry on physiological self-regulation depend on circumstances that facilitate cognitive emotion regulation. Study 2 aimed to further explore this possibility by showing the genocide film and making positive reappraisal either easier or more difficult by providing additional written information about the film.

\section{Study 2}

Study 2 builds on the findings from Study 1. We now tested the hypothesis that the findings in Study 1 were due to the ease with which participants engaged in cognitive reappraisal. In particular, we hypothesized that facilitating positive reappraisal after viewing the genocide film, where reappraisal is purportedly more difficult to apply, would change the effects of frontal asymmetry on physiological responses to reminder stimuli. Therefore, this study was similar in design to Study 1 , yet all participants were exposed to the genocide film, and only pictures from the genocide film were used as reminders in the startle paradigm. Furthermore, participants were provided with written information about the film that either encourages positive reappraisal or emphasizes negative aspects of the film. We measured engagement in positive reappraisal using an adapted state questionnaire. In the positive condition, we thus expected higher left-sided frontal activity to correlate with dampened startle potentiation after reminder picture offset, just as we found in the road accident condition in Study 1. Based the findings in study 1, we expected no or an opposite correlation in the negative condition.

In addition to reminder and neutral control pictures, we inserted unrelated negative pictures as a category to the startle paradigm. This was done to compare the effect of frontal asymmetry on responding to film reminders to its effect on responding to immediate affective provocation. Thus, similar to the findings of Jackson et al. (2003), we expected more left- 
sided frontal activity to predict dampened startle potentiation after unrelated negative image offset.

\section{Method}

\section{Participants}

Seventy-two healthy students (55 women) with a mean age of 20.9 years $(S D=2.1$; range: 18 - 30). Recruitment procedures, inclusion criteria, and compensation for participation were identical to Study 1. The study was approved by the standing ethical committee of the Faculty of Psychology and Neuroscience, Maastricht University.

\section{Procedure}

The procedure was kept similar to Study 1. All state and trait questionnaires used in Study 1 were included, and physiological recordings were identical. Again, not all questionnaire data are presented here ${ }^{2}$. After completion of baseline questionnaires and EEG measurements, all participants viewed the genocide film. They were then randomly assigned to read one of the two information texts regarding the film and given $5 \mathrm{~min}$ to read it attentively. An experimenter seated in an adjacent control room checked whether participants were reading via a CCTV system. When they finished reading earlier, they were asked to start over again. After that, the startle paradigm was administered. Participants were instructed to watch a series of pictures, some of which would be neutral, negative, or film-related. The instructions specified that while watching film-related images, participants should think of everything that they had learned during the film and by reading the debriefing. In order to assess engagement in emotion regulation, a state emotion regulation questionnaire was administered immediately following film viewing (i.e., before reading the information text) as well as following the startle paradigm. As well, emotional responses (i.e., PANAS, emotion VAS) were assessed once more following the startle paradigm to explore potential differences in emotional responding after reading the information texts.

\section{Materials and tasks}

Positive and negative information texts. The positive and negative information texts consisted of short articles (approximately 470 words) and contained background information

2 In Chapter 7, we present additional analyses involving some these questionnaire data. 
regarding the history of Rwanda related to the presented film fragments. In the positive condition, the text did not aim to down-play the seriousness of the events, but focused on positive aspects, such as that many individuals bravely stood up against the violence, organized rescue missions, or prevented extremists from killing. Furthermore, the text pointed out that the genocide was followed by legal and political investigations and reappraisals both within Rwanda and internationally, reflecting efforts to bring justice to the victims and to prevent similar events from happening in the future. In contrast, the text used in the negative condition repeated and complemented the horrible aspects depicted in the genocide documentary. This included the information that the involved ethnic groups had a longstanding and escalating conflict, resulting in extreme radicalization, hate, and eventually killings. The text further detailed the involvement of virtually all societal levels in the genocide, the inactivity of those who could have intervened, and the horrific numbers of victims who were killed or sexually abused.

Manipulation checks and control questions. To check whether participants in the two conditions differentially engaged in positive reappraisal following film viewing, we used an adapted state version of the Cognitive Emotion Regulation Questionnaire (CERQ; Garnefski, Kraaij, \& Spinhoven, 2002), which had been revised to tap into trauma film responses. The original CERQ is a 36-item scale that measures different cognitive emotion regulation strategies that individuals habitually use when confronted with negative events or situations. Each subscale consists of 4 items presenting a thought in form of a first-person statement (e.g., positive reappraisal: "I think that I can learn something from the situation."), and requiring respondents to indicate its frequency on a 5-point scale. In our adapted state version, respondents were asked to which degree they had used the specific strategies following exposure to the trauma film on 5-point scales $(1=$ very slightly or not at all, $5=$ extremely). For the purpose of this study, we used the positive reappraisal subscale only. At the administration following the startle paradigm (where item variance was the highest), its internal consistency was acceptable $(\alpha=.69)$, albeit somewhat lower than is typical for the respective trait subscale (Garnefski et al., 2002).

A number of control questions were given to the participants at the end of the session to control for prior knowledge of the shown documentary or details of its content. Furthermore, participants were asked to indicate the veracity of three simple statements concerning the content of the debriefing (true, untrue) to reassure good understanding content of the debriefing. One item was true for both debriefing conditions, whereas the other two items were true in one, but not the other debriefing condition. Due to technical failure, these data 
were lost for two participants. Among all other participants, four (5.7\%) failed to answer correctly on two of the three simple questions, raising doubts concerning their accurate understanding of the debriefings. Eight individuals indicated that they had known parts of the used trauma film prior to this study, and nine indicated that they had been familiar with details presented in this film. These numbers did not differ between the two conditions, Chi-Squares being $2.3(p=.13)$ and $0.1(p=.72)$, respectively. Excluding participants from the statistical analyses who either failed to answer the control questions correctly, or indicated being familiar with details beforehand, did not change the pattern of results and the conclusions.

Eye-blink startle paradigm. The pictures belonged to one of three categories: 30 reminder pictures from the genocide film, next to 30 neutral control (medium valence, low arousal ratings) and 30 negative control (low valence, high arousal ratings) pictures from the International Affective Picture System (IAPS; Lang et al., 2005). Intensity of the $50 \mathrm{~ms}$ white noise startle probes was set at $95 \mathrm{~dB}$.

\section{Data reduction}

Startle data from 2 participants ( 1 in each condition) were lost due to recording failure. Resting EEG data from 1 participant (positive condition) were excluded because only 9\% of epochs in the eyes-open condition were artefact-free. For all other participants, on average $84.3 \%($ range $=34-100 ; S D=14.9)$ of eyes-open and 97.3\% (range $=56-100 ; S D=6.9)$ of eyes-closed epochs were artefact-free. EEG data during film viewing were excluded from the analyses for 1 participant (negative condition), for whom less than $25 \%$ artefact-free epochs could be retained. For all other participants, on average $72.9 \%$ (range $=32-99 ; S D=18.2$ ) of all epochs $(N=1658)$ were artefact-free. Thus, data from 71 participants (35 in the positive condition) were available for analyses of resting frontal asymmetry, and from 70 (35 in the positive condition) for activation asymmetry.

\section{Statistical analysis}

Treatment of extreme cases, transformation of variables with skewed distributions, and the general statistical approach were identical to Study 1. State reappraisal scores were also log-transformed to reduce its strong right-skewed distribution. 


\section{Results}

\section{Resting and activation asymmetries}

As in Study 1, repeated measures ANOVAs showed no differences between resting and film-viewing frontal asymmetries, and no main or interaction effects involving debriefing condition, all $p \mathrm{~s}>.10$. As well, frontal activation asymmetry was unrelated to PANAS or VAS change scores, all $r \mathrm{~s}(70)<.12, p \mathrm{~s}>.27$. Again, resting frontal asymmetry scores $(M=$ $0.04, S D=0.09)$ highly correlated with asymmetry scores during film viewing $(M=0.05, S D$ $=0.10), r(70)=.80, p<.001$, but not with frontal activation asymmetries $(M=0.01, S D=$ $0.06), r=-.13, p=.30$.

\section{Emotional responses}

The two debriefing groups did not differ at baseline on any PANAS or emotion VAS subscale, all $t \mathrm{~s}(70)<1, p \mathrm{~s}>.35$. A 3 (Time: pre-film, post-film, end session) by 2 (Condition: positive, negative) repeated measures ANOVA on positive scores revealed a significant main effect of Time, $F(2,140)=72.8, p<.001, \eta 2 p=.51$, whereas no effects involving Condition were present, $F \mathrm{~s}<1$, $p \mathrm{~s}>.68$. Post-hoc pair-wise comparisons (Bonferroni) showed that positive affect decreased significantly from pre-film to post-film (Mean $\left._{\text {change }}=-1.8, S D=4.8\right)$ and decreased further from then to the end of the session $\left(\right.$ Mean $\left._{\text {change }}=-4.8, S D=4.5\right), p \mathrm{~s}<.01$. A similar 3 by 2 repeated measures ANOVA for negative affect revealed a significant main effect of Time, $F(2,140)=47.6, p<.001, \eta 2 p=$ .41 that did not interact with Condition, $p=.14$. Pair-wise comparisons showed an increase from pre-film to post-film (Mean change $=5.9, S D=6.1$ ), a decrease from then to the end of the session $(M=-3.5, S D=5.3)$, where scores were still significantly elevated with respect to baseline, $p \mathrm{~s}<.001$.

A 4 (Emotion: anxious, shocked, angry, sad) by 3 (Time) by 2 (Condition) repeated measures ANOVA showed a significant Emotion by Time interaction, $F(6,420)=32.8, p<$ .001 , Epsilon $=.86, \eta 2 p=.32$, next to main effects of Emotion, $F(3,210)=13.9, p<.001$, Epsilon $=.80, \eta 2 p=.17$, and Time, $F(2,140)=78.2, p<.001, \eta 2 p=.53$. There were no main or interaction effects involving Condition, $p s>.50$. Pair-wise comparisons (Bonferroni) between timings showed that feeling shocked, angry, and sad increased in response to film viewing $\left(\right.$ Mean $_{\text {change }}=37.8,35.2$, and 28.3, respectively; $S D=30.5,31.1$, and 28.2), then decreased again, yet remaining elevated at the end of the session with respect to baseline, all $p s<.001$. Scores on feeling anxious were much lower overall $\left(\right.$ Mean $\left._{\text {change }}=5.4, S D=19.3\right)$, 
yet a comparable pattern was observed, with both post-film and end-session scores marginally differing from baseline, $p=.047$ and $p=.062$.

\section{Positive reappraisal following the debriefings}

To test whether the two debriefing conditions affected the use of positive reappraisal in the period following film viewing, positive reappraisal scores of the state CERQ subscales at the end of the sessions were entered as dependent variables in an ANCOVA, using condition as independent variable and CERQ score prior to reading the positive or information as covariate. The covariate was a significant predictor, $F \mathrm{~s}(1,69)=44.5, p<.001, \eta 2 p=.39$, and, as intended, condition significantly affected positive reappraisal, $F(1,69)=5.3, p=$ $.024, \eta 2 p=.07$, with a larger increase following the positive information $\left(M_{p r e}=6.2, S D=\right.$ $\left.1.8 ; M_{\text {post }}=6.8, S D=2.3\right)$ than following the negative information $\left(M_{\text {pre }}=6.5, S D=1.9 ; M_{\text {post }}\right.$ $=6.1, S D=2.3), t(70)=2.5, p=.016$.

\section{Condition effects and time course of startle potentiation}

A 2 (Category: reminder, negative) by 3 (Probe Timing: 2.5, 4.5, $7 \mathrm{~s}$ ) by 2 (Debriefing Condition: positive, negative) repeated measures ANOVA on startle potentiation scores revealed no significant three-way interaction, $F(2,136)<1, p=.57$. However, there was a significant Probe Timing by Condition interaction, $F(2,136)=3.9, p=.022, \eta 2 p=.06$, next to a main effect for Category, $F(1,68)=18.9, p<.001, \eta 2 p=.22$, indicating that startle potentiation scores were significantly larger in negative compared to reminder pictures. Follow-up analyses revealed that in the positive debriefing condition, only Category significantly modulated startle potentiation, $F(1,34)=19.8, p<.001, \eta 2 p=.37$, with higher scores for negative, compared to reminder trials. In the negative debriefing condition, the effect of Category was reduced to trend-significant level, $F(1,34)=3.7, p=.064, \eta 2 p=.10$, and a significant main effect of Time emerged, $F(2,68)=5.6, p=.006, \eta 2 p=.14$. Pair-wise comparisons (Bonferroni) on estimated marginal means in this condition showed that potentiation scores at the $4.5 \mathrm{~s}$ timing were higher than at the $2.5 \mathrm{~s}$ timing, $p=.012$, and also tended to be higher than at the $7 \mathrm{~s}$ timing, $p=.091$, irrespective of Category. 


\section{Frontal asymmetry effects on startle potentiation ${ }^{3}$}

For resting frontal asymmetry effects on startle potentiation after reminder picture offset, a regression model $(d f=3,65)$ showed no significant moderation by debriefing condition, $p=.70$. Correlation analyses in the entire sample revealed a significant positive correlation between resting frontal asymmetry and startle potentiation, $r(69)=.33, p=.005$, with more left-sided frontal activity predicting higher startle potentiation after reminder picture offset. For activation asymmetry as a predictor, the regression model $(d f=3,64)$ similarly revealed a non-significant interaction term, $p=.50$. Unlike resting asymmetry, it did not correlate significantly across the entire sample with startle potentiation after reminder picture offset, $r(68)=-.02, p=.89$ (see Figure 5.5). In order to determine whether resting frontal asymmetry predicted startle potentiation over and above emotional responses to the trauma film, we included anger and sadness increase as predictors, followed by entering resting frontal asymmetry. This second step significantly enhanced the model, $R 2_{\text {change }}=.06$, $F(1,65)=4.9, p=.03$, resting frontal asymmetry being a significant predictor, $\beta=.26, t=$ $2.2, p=.03$.

Further exploratory analyses revealed no interaction effects or correlations for startle potentiation at the other probe timings or after unrelated negative picture offset involving frontal resting, $r \mathrm{~s}(69)<.08, p \mathrm{~s}>.55$, or activation asymmetry, $r \mathrm{~s}(68)<.18, p \mathrm{~s}>.15$. For parietal asymmetry at rest, we found an interaction at trend level $(p=.05)$, but no significant correlations in both debriefing groups, $p \mathrm{~s}>.13$. For parietal activation asymmetry, there was no significant interaction, $p>.34$, and no significant zero-order correlation, $p=.07$.

3 PANAS and emotion VAS change scores, as well as positive reappraisal scores, were unrelated to reminder startle potentiation, largest $r=-.19, p=.11$, and are therefore omitted from the following analyses. However, we considered anger and sadness increase scores in the following models, as they correlated positively with startle potentiation scores at the $7 \mathrm{~s}$ timing for reminder trials, $r=.33, p<.01$, and $r=.27, p=.03$. 


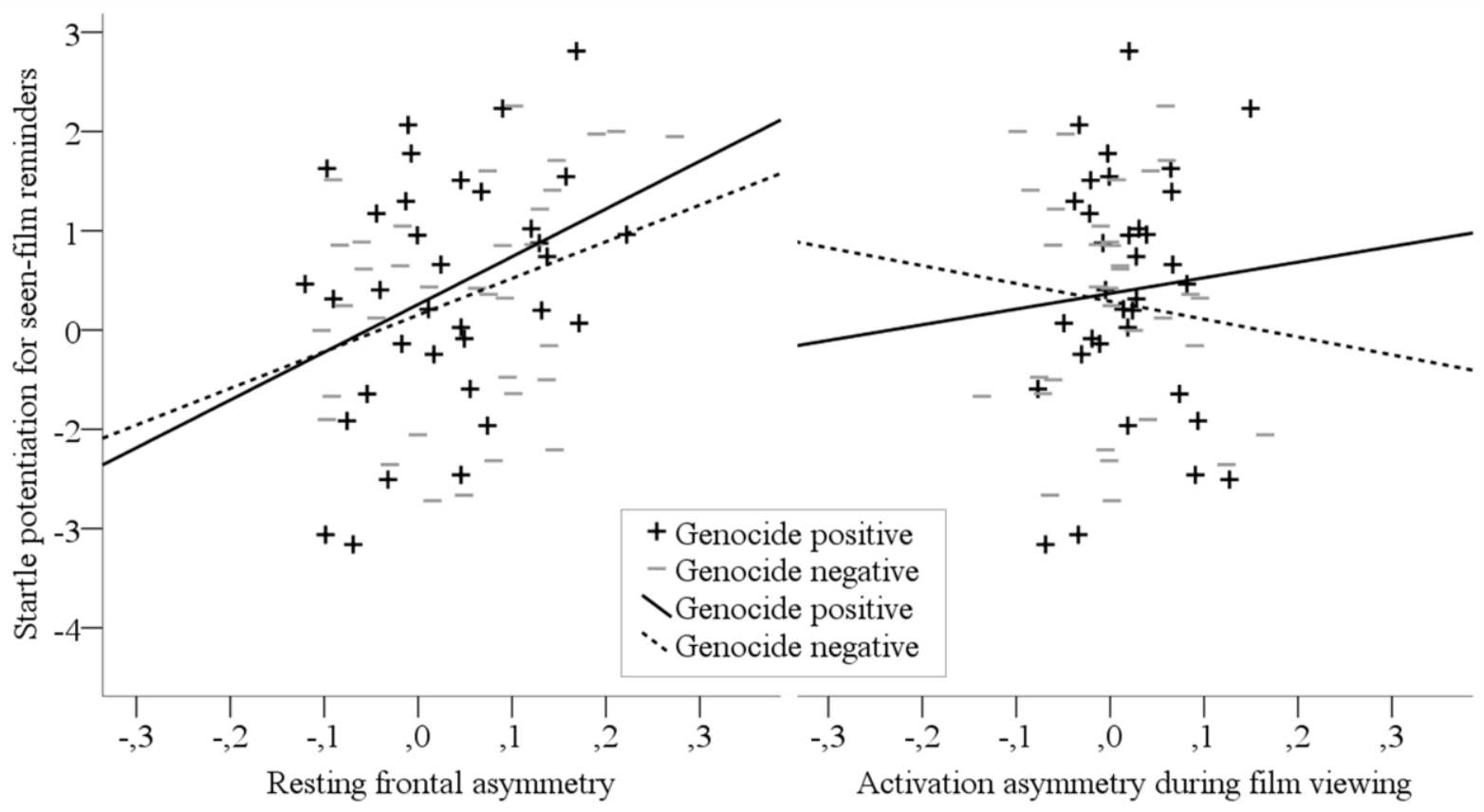

Figure 5.5. Scatter-plots depicting frontal asymmetry at rest (left panel; $N=69$ ) and activation asymmetry during film viewing (right panel; $N=68$ ) as predictors of startle potentiation at $7 \mathrm{~s}(1 \mathrm{~s}$ post-stimulus offset) for film reminders. Both interactions involving information condition are not significant. Across groups, resting frontal asymmetry positively predicted reminder startle potentiation, whereas no significant associations emerged with activation asymmetry.

\section{Summary and comparison with study 1}

The results indicate that as intended, the positive information condition facilitated the use of reappraisal compared to the negative information condition. Yet, this appeared to have no effect on emotional responding. Regarding the effects of frontal asymmetry on physiological self-regulation in response to trauma-film reminders, condition did not play a moderating role. However, we now found that more left-sided frontal activity at rest predicted higher startle potentiation after reminder picture offset (i.e., worse physiological selfregulation) in both conditions, over and above increases in feeling angry and sad due to film viewing that were similarly related to startle. Meanwhile, frontal activation asymmetry was unrelated to startle outcomes. Furthermore, we found no relationship between frontal asymmetry and startle potentiation after the offset of unrelated negative pictures, which appears to be at odds with previous findings by Jackson et al. (2003). One explanation could be that affective processing in negative images was not independent from processing reminder images. This interpretation seems to be supported by our finding that the time course of startle 
potentiation was modulated by debriefing condition in the absence of an interaction with stimulus category. That is, in the context of our startle paradigm, unrelated negative pictures may also have activated thoughts about the previously seen trauma film, which may have impeded emotion regulation in response to these pictures.

The finding that in Study 1 frontal activation asymmetry, but not resting asymmetry, predicted startle regulation (differently for the two trauma films) appears to be at odds with the finding in Study 2 that resting frontal asymmetry, but not activation asymmetry predicted startle regulation. Since resting frontal asymmetry may be susceptible to confounding by transient motivational factors (Coan et al., 2006), we explored the possibility that resting asymmetry reflected a state of subtle emotional provocation in Study 2, but not in Study 1 . We thus compared baseline (i.e., pre-film) affective states between the two studies and explored their influence on the resting measurement of frontal asymmetry. Baseline PA and NA levels did not differ between the two studies, $t$ s $(134)<1$, ps $>.41$. Likewise, a 4 (Emotion) by two (Study) repeated measures ANOVA on baseline emotion VAS scores yielded no significant main or interaction effects involving Study, all $p s>$.13. Also resting frontal asymmetry levels were similar in both studies, $t(131)=1.1, p=.28$. In Study 1 , resting frontal asymmetry did not correlate with any of the baseline indices of current emotion, all $r \mathrm{~s}(62)<.18, p \mathrm{~s}>.18$. Only in study 2 , more left-sided frontal activity at rest tended to correlate with lower baseline anxiety levels, $r(71)=-.23, p=.06$.

\section{Discussion}

\section{Type of trauma film moderates the effects}

Across two studies, the most salient observation is that frontal asymmetry appears to have distinct effects on physiological self-regulation in the activation of aversive memories, depending on the type of aversive material used. While more left-sided frontal activity or activation was hypothesized to predict dampened startle responses after reminder picture offset, this was only the case for reminders of a film depicting a staged road accident. In contrast, for reminders of a genocide documentary, left-sided activation during film viewing tended to predict stronger, rather than dampened, startle responses in Study 1, and we found a similar and significant effect for left-sided resting activity in Study 2. Thus, our findings indicate that frontal asymmetry at rest and during provocation can be associated with both enhanced and impaired physiological self-regulation. Notably, the positive and negative correlations were in the .30-.50 range and, thus, comparable in size to the effects reported by 
Jackson et al. (2003). These authors found that left-sided frontal activity at rest predicted lower startle potentiation after negative picture offset. Furthermore and in line with the view that frontal asymmetry indexes individual differences in automatic emotion regulation (Jackson et al., 2003; Parvaz et al., 2012), its effects manifested specifically after reminder picture offset, whereas it did not significantly predict physiological responses during picture presentation. As well, our data show that these effects were specific to the anterior electrode pairs, and were not present in parietal electrodes.

What might account for the differential effects of frontal asymmetry and activation in the two film conditions? An appealing interpretation is that differences in content-related cognitive processes may have resulted in different consequences of attempts to regulate emotions. Compared to the road-accident film, emotion regulation in the genocide film may have required a higher number of cognitive resources, which is thought to have deleterious consequences for physiological responding (Gross \& John, 2003). This might explain why we found a link between more left-sided frontal activity and higher responding to film reminders. Study 2 aimed to explore the role of one such process, namely positive reappraisal, by which individuals alter the emotional impact of a stressor by attaching a positive meaning (Garnefski et al., 2002). Although we succeeded in manipulating positive reappraisal by providing positive and negative information, this had no effect on the association between frontal asymmetry and physiological responding. These findings could indicate that reappraisal is not the crucial moderator that we tried to identify.

However, it would be premature to rule out this possibility. Importantly, providing positive information that people can use for positive reappraisal may have been a relatively weak intervention. As a consequence, even in the positive condition, participants may still have been unable to engage in positive reappraisal successfully. This view is supported by the absence of condition differences in emotional recovery following film viewing. It is therefore possible that participants in the positive debriefing condition of Study 2 used positive reappraisal still to very little extent, especially when compared with our participants in the road accident condition of Study 1. Since we did not collect data regarding emotion regulation in Study 1, this issue remains to be investigated further. Future studies might thus want to use emotional material in which reappraisal is easier to influence, or use stronger manipulations, such as directly challenging participants' cognitive and emotional reactions (e.g., Takarangi \& Strange, 2010).

Furthermore, there may be differences between the films that were not measured in this study. For instance, the used road-accident film had a positive educational message that 
participants can integrate with their prior knowledge about road safety. In contrast, the genocide film consisted of real-life footage aiming to inform and educate about a historical humanitarian and political tragedy. It is very unlikely that our (western-European) participants were directly confronted with the horrors of genocide, and they may encounter difficulties to integrate this content with existing knowledge and prior beliefs. Notably, such difficulties are thought to exacerbate the risk of developing psychopathology after trauma (Ehlers \& Clark, 2000; Janoff-Bulman, 1992), and could provide an alternative account for why left-sided frontal activity was associated with exaggerated startle responding in Study 2.

\section{Effects of Resting and State-Dependent Frontal Asymmetry}

Another salient observation in the present studies is that physiological self-regulation could be predicted by resting frontal asymmetry in Study 2, whereas frontal activation asymmetry during film viewing was predictive in Study 1. This finding is surprising, since the measurement of resting and film-related frontal asymmetry were kept similar in both studies. A possible explanation is that individual differences in frontal asymmetry may only be predictive of emotional outcomes if they are measured in a relevant motivational context. For example, frontal asymmetry correlates with negative affectivity when measured in a fearful state (Coan et al., 2006) or with trait approach motivation when measured in a state of sexual attraction (Wacker, Mueller, Pizzagalli, Hennig, \& Stemmler, 2013). According to this view, our resting state measurements can be considered as an uncontrolled motivational context, which may have been relevant for the later responses to the trauma films (e.g., anticipatory anxiety, nervousness) or not (e.g., curiosity, feeling bored). If the resting state reflected such a "relevant" emotional context for many participants in Study 2, but not in Study 1, then this would explain why resting asymmetry predicted physiological self-regulation in reminder pictures in Study 2, but not in Study 1. Consequentially, change in frontal asymmetry during film viewing may have been more relevant to emotional responding in Study 1, compared to Study 2.

Note that this explanation remains speculative, since our data do not provide compelling indications that the resting state measurements were differentially influenced by emotional states. That is, we found no robust differences in baseline motivational states or in asymmetry scores. Also, there were only weak indications (i.e., a relatively small trend) that pre-film anxiety may have led to reduced left-sided resting activity in Study 2 only. Thus, with the present data, we can only conclude that frontal asymmetry measured at rest and during 
provocation can both be informative about individual differences in responding to trauma-film reminders. This confirms the utility of including both baseline measurements and standardized provocations to capture relevant individual differences in frontal asymmetry.

One of the practical questions raised in this article was whether frontal asymmetry could serve to predict resilient responding at a later point in time. While our models including resting frontal asymmetry provide direct evidence for answering this question, the models including film-induced activation asymmetry must be interpreted with more caution. On the one hand, activation asymmetry can be conceptualized as tapping the individual's trait-like capability of emotional responding, and thus would be thought to moderate later physiological responses. On the other hand, our measurement of activation asymmetry is intrinsically linked with responding to the trauma film, and may thus itself reflect a (mal)adaptive process that mediates later physiological responses (see Figure 5.2, panel B). Our findings favour the moderation over the mediation model, since frontal asymmetry was not systematically affected by film viewing and did not correlate with subjective indices of emotional responding to the film. Of note, in light of the unexpected moderation by film condition in Study 1, it is possible that a small mediation effect occurred in the road accident clip only, which may have gone undetected due to a lack of power. Thus, to disentangle these interpretations more conclusively, future studies would need to include a separate emotional provocation before trauma-film viewing and test whether pre-film and peri-film activation asymmetries display patterns similar to those found here.

\section{Limitations}

There are some caveats to interpretation of the present data that deserve to be mentioned. First, in Study 1, the victims in the two trauma films differed in ethnicity, which might have led to stronger in-group empathy, and hence emotional responding in the road accident condition (Brown, Bradley, \& Lang, 2006). This might provide an alternative account for differential effects in the two film conditions. However, in both films, we clearly observed that the used films produced the intended subjective responses, including a reduction in PA paralleled by increases in NA, as well as increased VAS scores on feeling shocked, angry, and sad. All of these effects were more pronounced in the genocide film than the road accident film, which makes in-group empathy an unlikely confounding factor in the present data. Second, and conversely, higher mean emotionality in the genocide condition may have led to a greater need for emotion regulation in this condition. Likewise, across participants, 
our data from Study 1 suggest enhanced responding to seen-film reminder pictures (compared to unseen-film reminders) only in the genocide film condition. However, it appears plausible that these condition effects are the result, rather than the antecedent, of differences in emotion regulation processes. The absence of heightened startle potentiation in the road-accident condition can be interpreted in terms of successful physiological down-regulation. Third, our findings may be limited by the parameters and stimuli that we used in our startle paradigm. For instance, we assessed physiological self-regulation using startle probes $1 \mathrm{~s}$ after stimulus offset. Research indicates that this timing is well-suited to capture sustained affective processing after picture offset (Hajcak \& Olvet, 2008), and modulation of physiological responses by emotion regulation can occur even faster, in the range of several $100 \mathrm{~ms}$ (e.g., Blechert, Sheppes, Di Tella, Williams, \& Gross, 2012). Still, our data are not informative regarding regulatory effects that may manifest themselves in the range of minutes, hours, and longer. Another concern may be that in Study 2, startle potentiation was lower in film reminders compared to unrelated negative images. Although this is not surprising, it also indicates that the responses to trauma-film memories may not be comparable to those seen in traumatized individuals, for whom trauma memories can be highly distressing. Finally, a related concern is that our results are limited by the use of a rather homogenous, highfunctioning student sample in that they may not translate directly to clinical populations.

\section{Conclusion}

We investigated whether indices of frontal EEG asymmetry can serve as a predictor of physiological responding to aversive memories. This was done by subjecting participants to a trauma-film paradigm and administering a startle paradigm in which they saw reminder images from the film they had seen. Both resting frontal asymmetry and frontal activation asymmetry in response to film viewing served as predictors of startle responses. Our results suggest that relatively left-sided frontal activity at rest and activation during viewing of an aversive film can inform about individual differences in the processing of aversive memories, but they can be predictive of enhanced as well as of impaired physiological self-regulation, depending on the type of trauma film that is used. We found no indication that engagement in positive reappraisal is responsible for these differential effects, but future studies are clearly needed to further explore this possibility. Furthermore, our findings call for a systematic investigation of other potential moderators in the effects of frontal asymmetry, such as the availability of contextual information, prior knowledge, beliefs and schemas. Eventually, this 
might contribute to our understanding of individual risk and resilience factors and their differential involvement in different types of traumatic experience (e.g., Grimm, Hulse, Preiss, \& Schmidt, 2012; McNally \& Robinaugh, 2011).

\section{Author Note}

This study was supported in part by Grant 056-25-011 from the Netherlands Organization for Scientific Research (NWO) to Dr. Tom Smeets. Parts of these data have been presented at the $13^{\text {th }}$ ESTSS conference in Bologna, Italy. We are especially thankful to Sarah Baumann, Saira Berberovic, Hans Keulers, Sarah Mauder, Andrea Nagel, Ingrid Rooijakkers, and Maxi Volk, for their help in collecting the data for study 1 . 


\section{References}

Allen, J. J. B., Coan, J. A., \& Nazarian, M. (2004). Issues and assumptions on the road from raw signals to metrics of frontal EEG asymmetry in emotion. Biological Psychology, 67, 183-218. doi: 10.1016/j.biopsycho.2004.03.007

Allen, J. J. B., \& Kline, J. P. (2004). Frontal EEG asymmetry, emotion, and psychopathology: The first, and the next 25 years. Biological Psychology, 67, 1-5. doi: 10.1016/j.biopsycho.2004.03.001

American Electroencephalographic Society. (1994). Guideline thirteen: Guidelines for standard electrode position nomenclature. Journal of Clinical Neurophysiology, 11, 111-113.

Blechert, J., Sheppes, G., Di Tella, C., Williams, H., \& Gross, J. J. (2012). See what you think: Reappraisal modulates behavioral and neural responses to social stimuli. Psychological Science, 23, 346-353. doi: 10.1177/0956797612438559

Blumenthal, T. D., Cuthbert, B. N., Filion, D. L., Hackley, S., Lipp, O. V., \& Van Boxtel, A. (2005). Committee report: Guidelines for human startle eyeblink electromyographic studies. Psychophysiology, 42, 1-15. doi: 10.1111/j.1469-8986.2005.00271.x

Boden, M. T., Westermann, S., McRae, K., Kuo, J., Alvarez, J., Kulkarni, M. R., . . BonnMiller, M. O. (2013). Emotion regulation and posttraumatic stress disorder: A prospective investigation. Journal of Social and Clinical Psychology, 32, 296-314.

Bonanno, G. A. (2004). Loss, trauma, and human resilience - Have we underestimated the human capacity to thrive after extremely aversive events? American Psychologist, 59, 20-28. doi: 10.1037/0003-066x.59.1.20

Brewin, C. R., Gregory, J. D., Lipton, M., \& Burgess, N. (2010). Intrusive images in psychological disorders: Characteristics, neural mechanisms, and treatment implications. Psychological Review, 117, 210-232. doi: 10.1037/a0018113

Brown, L. M., Bradley, M. M., \& Lang, P. J. (2006). Affective reactions to pictures of ingroup and outgroup members. Biological Psychology, 71, 303-311. doi: 10.1016/j.biopsycho.2005.06.003

Coan, J. A., \& Allen, J. J. B. (2004). Frontal EEG asymmetry as a moderator and mediator of emotion. Biological Psychology, 67, 7-49. doi: 10.1016/j.biopsycho.2004.03.002

Coan, J. A., Allen, J. J. B., \& McKnight, P. E. (2006). A capability model of individual differences in frontal EEG asymmetry. Biological Psychology, 72, 198-207. doi: 10.1016/j.biopsycho.2005.10.003 
Davidson, R. J. (1998). Affective style and affective disorders: Perspectives from affective neuroscience. Cognition \& Emotion, 12, 307-330.

Davis, M., Walker, D. L., \& Lee, Y. L. (1997). Roles of the amygdala and bed nucleus of the stria terminalis in fear and anxiety measured with the acoustic startle reflex - Possible relevance to PTSD. Annals of the New York Academy of Sciences, 821, 305-331. doi: 10.1111/j.1749-6632.1997.tb48289.x

Ehlers, A., \& Clark, D. M. (2000). A cognitive model of posttraumatic stress disorder. Behaviour Research and Therapy, 38, 319-345. doi: 10.1016/S0005-7967(99)00123-0

Garnefski, N., Kraaij, V., \& Spinhoven, P. (2002). Manual for the use of the Cognitive Emotion Regulation Questionnaire. Leiderdorp, The Netherlands: DATEC.

Goldsmith, H. H., \& Davidson, R. J. (2004). Disambiguating the components of emotion regulation. Child Development, 75, 361-365.

Goodman, R. N., Rietschel, J. C., Lo, L.-C., Costanzo, M. E., \& Hatfield, B. D. (2013). Stress, emotion regulation and cognitive performance: The predictive contributions of trait and state relative frontal EEG alpha asymmetry. International Journal of Psychophysiology, 87, 115-123. doi: 10.1016/j.ijpsycho.2012.09.008

Grimm, A., Hulse, L., Preiss, M., \& Schmidt, S. (2012). Post- and peritraumatic stress in disaster survivors: An explorative study about the influence of individual and event characteristics across different types of disasters. European Journal of Psychotraumatology, 3. doi: 10.3402/ejpt.v3i0.7382

Gross, J. J., \& John, O. P. (2003). Individual differences in two emotion regulation processes: Implications for affect, relationships, and well-being. Journal of Personality and Social Psychology, 85, 348-362. doi: 10.1037/0022-3514.85.2.348

Hajcak, G., \& Olvet, D. M. (2008). The persistence of attention to emotion: Brain potentials during and after picture presentation. Emotion, 8, 250-255. doi: 10.1037/15283542.8.2.250

Harmon-Jones, E., Gable, P. A., \& Peterson, C. K. (2010). The role of asymmetric frontal cortical activity in emotion-related phenomena: A review and update. Biological Psychology, 84, 451-462.

Heller, W. (1993). Neuropsychological mechanisms of individual differences in emotion, personality, and arousal. Neuropsychology, 7, 476-489.

Holmes, E. A., \& Bourne, C. (2008). Inducing and modulating intrusive emotional memories: A review of the trauma film paradigm. Acta Psychologica, 127, 553-566. doi: 10.1016/j.actpsy.2007.11.002 
Holmes, E. A., James, E. L., Coode-Bate, T., \& Deeprose, C. (2009). Can playing the computer game "Tetris" reduce the build-up of flashbacks for trauma? A proposal from cognitive science. Plos One, 4, 6. doi: e415310.1371/journal.pone.0004153

Jackson, D. C., Mueller, C. J., Dolski, I., Dalton, K. M., Nitschke, J. B., Urry, H. L., . . . Davidson, R. J. (2003). Now you feel it, now you don't: Frontal brain electrical asymmetry and individual differences in emotion regulation. Psychological Science, $14,612-617$.

Janoff-Bulman, R. (1992). Shattered assumptions: Toward a new psychology of trauma. New York: The Free Press.

Keil, A., Debener, S., Gratton, G., Junghöfer, M., Kappenman, E. S., Luck, S. J., . . Yee, C. M. (2013). Committee report: Publication guidelines and recommendations for studies using electroencephalography and magnetoencephalography. Psychophysiology, n/an/a. doi: 10.1111/psyp.12147

Koslov, K., Mendes, W. B., Pajtas, P. E., \& Pizzagalli, D. A. (2011). Asymmetry in resting intracortical activity as a buffer to social threat. Psychological Science, 22, 641-649. doi: $10.1177 / 0956797611403156$

Lang, P. J., Bradley, M. M., \& Cuthbert, B. N. (2005). International Affective Picture System (IAPS): Instruction manual and affective ratings. Technical report A-6. Gainesville, FL: University of Florida.

Lopez-Duran, N. L., Nusslock, R., George, C., \& Kovacs, M. (2012). Frontal EEG asymmetry moderates the effects of stressful life events on internalizing symptoms in children at familial risk for depression. Psychophysiology, 49, 510-521. doi: 10.1111/j.14698986.2011.01332.x

McNally, R. J., \& Robinaugh, D. J. (2011). Risk factors and posttraumatic stress disorder: Are they especially predictive following exposure to less severe stressors? Depression and Anxiety, 28, 1091-1096. doi: 10.1002/da.20867

Meyer, T., Smeets, T., Giesbrecht, T., \& Merckelbach, H. (2012). The efficiency of reappraisal and expressive suppression in regulating everyday affective experiences. Psychiatry Research, 200, 964-969. doi: 10.1016/j.psychres.2012.05.034

Meyer, T., Smeets, T., Giesbrecht, T., Quaedflieg, C. E. M., Girardelli, M., Mackay, G. N., \& Merckelbach, H. (2013). Individual differences in spatial configuration learning predict the occurrence of intrusive memories. Cognitive, Affective, \& Behavioral Neuroscience, 13, 186-196. doi: 10.3758/s13415-012-0123-9 
Miller, G. A., Crocker, L. D., Spielberg, J. M., Infantolino, Z. P., \& Heller, W. (2013). Issues in localization of brain function: The case of lateralized frontal cortex in cognition, emotion, and psychopathology. Frontiers in Integrative Neuroscience, 7. doi: 10.3389/fnint.2013.00002

Nusslock, R., Shackman, A. J., Harmon-Jones, E., Alloy, L. B., Coan, J. A., \& Abramson, L. Y. (2011). Cognitive vulnerability and frontal brain asymmetry: Common predictors of first prospective depressive episode. Journal of Abnormal Psychology, 120, $497-$ 503. doi: $10.1037 / \mathrm{a} 0022940$

Orr, S. P., \& Roth, W. T. (2000). Psychophysiological assessment: Clinical applications for PTSD. Journal of Affective Disorders, 61, 225-240.

Ozer, E. J., Best, S. R., Lipsey, T. L., \& Weiss, D. S. (2003). Predictors of posttraumatic stress disorder and symptoms in adults: A meta-analysis. Psychological Bulletin, 129, 52-73. doi: 10.1037//0033-2909.129.1.52

Parvaz, M., MacNamara, A., Goldstein, R., \& Hajcak, G. (2012). Event-related induced frontal alpha as a marker of lateral prefrontal cortex activation during cognitive reappraisal. Cognitive, Affective, \& Behavioral Neuroscience, 12, 730-740. doi: 10.3758/s13415-012-0107-9

Pfurtscheller, G., Stancak, A., \& Neuper, C. (1996). Event-related synchronization (ERS) in the alpha band - An electrophysiological correlate of cortical idling: A review. International Journal of Psychophysiology, 24, 39-46. doi: 10.1016/s01678760(96)00066-9

Pivik, R. T., Broughton, R. J., Coppola, R., Davidson, R. J., Fox, N., \& Nuwer, M. R. (1993). Guidelines for the recording and quantitative analysis of electroencephalographic activity in research contexts. Psychophysiology, 30, 547-558. doi: 10.1111/j.14698986.1993.tb02081.x

Rivest, L. P. (1994). Statistical properties of Winsorized means for skewed distributions. Biometrika, 81, 373-383. doi: 10.1093/biomet/81.2.373

Takarangi, M. K. T., \& Strange, D. (2010). Emotional impact feedback changes how we remember negative autobiographical experiences. Experimental Psychology, 57, 354359. doi: 10.1027/1618-3169/a000042

Thibodeau, R., Jorgensen, R. S., \& Kim, S. (2006). Depression, anxiety, and resting frontal EEG asymmetry: A meta-analytic review. Journal of Abnormal Psychology, 115, 715729. doi: 10.1037/0021-843x.115.4.715 
Tomarken, A. J., Davidson, R. J., Wheeler, R. E., \& Doss, R. C. (1992). Individual differences in anterior brain asymmetry and fundamental dimensions of emotion. Journal of Personality and Social Psychology, 62, 676-687.

Tomarken, A. J., Davidson, R. J., Wheeler, R. E., \& Kinney, L. (1992). Psychometric properties of resting anterior EEG asymmetry: Temporal stability and internal consistency. Psychophysiology, 29, 576-592.

Towers, D. N., \& Allen, J. J. B. (2009). A better estimate of the internal consistency reliability of frontal EEG asymmetry scores. Psychophysiology, 46, 132-142. doi: 10.1111/j.1469-8986.2008.00759.x

Velo, J. R., Stewart, J. L., Hasler, B. P., Towers, D. N., \& Allen, J. J. B. (2012). Should it matter when we record? Time of year and time of day as factors influencing frontal EEG asymmetry. Biological Psychology, 91, 283-291.

Wacker, J., Mueller, E. M., Pizzagalli, D. A., Hennig, J. r., \& Stemmler, G. (2013). Dopamine-D2-receptor blockade reverses the association between trait approach motivation and frontal asymmetry in an approach-motivation context. Psychological Science, 24, 489-497. doi: 10.1177/0956797612458935

Watson, D., Clark, L. A., \& Tellegen, A. (1988). Development and validation of brief measures of positive and negative affect: The PANAS scales. Journal of Personality and Social Psychology, 54, 1063-1070.

Weidmann, A., Conradi, A., Grögera, K., Fehma, L., \& Fydrich, T. (2009). Using stressful films to analyze risk factors for PTSD in analogue experimental studies - Which film works best? Anxiety, Stress \& Coping, 22, 549-569. 


\section{Chapter 6}

\section{The role of frontal EEG asymmetry in post-traumatic stress disorder}

An adapted version of this Chapter is published as:

Meyer, T., Smeets, T., Giesbrecht, T., Quaedflieg, C. W. E. M., Smulders, F., Meijer, E., \& Merckelbach, H. (in press). The role of frontal EEG asymmetry in post-traumatic stress disorder. Biological Psychology. 



\section{Abstract}

Frontal alpha asymmetry, a biomarker derived from electroencephalography (EEG) recordings, has often been associated with psychological adjustment, with more left-sided frontal activity predicting approach motivation and lower levels of depression and anxiety. Therefore, frontal asymmetry might be relevant to post-traumatic stress disorder (PTSD), a disorder comprising anxiety and dysphoria symptoms. We review this relationship and show that frontal asymmetry can be plausibly linked to neuropsychological abnormalities seen in PTSD. However, surprisingly few studies $(k=8)$ have directly addressed frontal asymmetry in PTSD, mostly reporting that trait frontal asymmetry has little (if any) predictive value for PTSD symptoms. Meanwhile, preliminary evidence suggest that state-dependent asymmetry during trauma-relevant stimulation distinguishes PTSD patients from resilient individuals. Thus, exploring links between provocation-induced EEG asymmetry and PTSD appears particularly promising. Additionally, we recommend more fine-grained analyses of PTSD symptom clusters in relation to frontal asymmetry. Finally, we highlight hypotheses that may guide future research and help to fully apprehend the practical and theoretical relevance of this biological marker.

\section{Key words}

Frontal EEG asymmetry; cerebral lateralization; post-traumatic stress disorder; depression; anxiety 


\section{Introduction}

At some point in their life, many people are exposed to potentially traumatic events, such as the death of a close friend, violent crimes, and severe accidents. Most people seem to be able to adapt well to aversive experiences (Bonanno, 2004, 2012; Bonanno \& Mancini, 2008), but a significant proportion develop post-traumatic stress disorder (PTSD). Victims with PTSD suffer from prolonged reactions to the traumatic event, including re-experiencing (e.g., intrusions, nightmares), avoidance of cues related to the trauma, altered mood and cognition, as well as exaggerated general arousal and reactivity (American Psychiatric Association, 2013). Strikingly, the severity of these symptoms is only modestly related to the objective severity of the traumatic event (Brewin, Andrews, \& Valentine, 2000; Kessler, Sonnega, Bromet, Hughes, \& Nelson, 1995; D. G. Kilpatrick, Resnick, \& Acierno, 2009; McNally \& Robinaugh, 2011). Therefore, researchers and clinicians alike have been intrigued by the question of what characterizes people who suffer from pathological symptoms after adversity, and whether there are biological markers to objectively measure these individual characteristics.

The search for objective indicators of PTSD is pressing for various reasons (Lehrner \& Yehuda, 2014; Zoellner, Bedard-Gilligan, Jun, Marks, \& Garcia, 2013). For instance, the diagnosis of this disorder essentially relies on self-report (Rosen \& Lilienfeld, 2008), and objective markers could be used for the development of more reliable diagnostic tests, with important implications for clinical and legal practice (Zoellner et al., 2013). Also, research into biological markers of PTSD helps to develop indicators of prognosis or treatment outcome, as well as preventive interventions for high-risk groups (Lehrner \& Yehuda, 2014). This field of research has seen considerable progress in the recent years (for reviews, see: U. Schmidt, Kaltwasser, \& Wotjak, 2013; Zoladz \& Diamond, 2013). For instance, PTSD has been linked to increased stress hormone levels (Inslicht et al., 2011), stress hormone signalling (van Zuiden et al., 2012), physiological reactivity (Pole, 2007; Pole et al., 2009), or reduced extinction of conditioned fear (Lommen, Engelhard, Sijbrandij, van den Hout, \& Hermans, 2013).

The present article will review literature on so-called frontal asymmetry as a potential objective indicator of PTSD symptoms following trauma exposure. Frontal asymmetry is a widely studied biomarker in research on emotional and behavioural reactions to stressful situations. It refers to a difference in mean alpha band power (typically $8-13 \mathrm{~Hz}$ ) between the left and right frontal cortex over a time span of several minutes, and is usually measured using 
electroencephalography (EEG) (Coan \& Allen, 2003). A widespread interpretation of frontal asymmetry is based on the assumption that alpha band power is inversely related to brain activity, implying that frontal asymmetry in alpha power reflects hemispheric differences in frontal brain idling (Pfurtscheller, Stancak, \& Neuper, 1996) (though see Buzsaki \& Draguhn, 2004; Klimesch, Sauseng, \& Hanslmayr, 2007).

Coan and Allen (2004) and more recently, Harmon-Jones, Gable, and Peterson (2010) summarized the many EEG studies that successfully used frontal asymmetry as a psychophysiological marker of affect-related traits, or as a predictor of acute affective regulation. The interest in this marker is hardly on the wane, with a large and growing body of evidence indicating that relatively higher left-sided frontal brain activity is associated with superior affect and stress regulation (e.g., Koslov, Mendes, Pajtas, \& Pizzagalli, 2011; Quaedflieg, Meyer, Smulders, \& Smeets, 2015; Tomarken, Davidson, Wheeler, \& Doss, 1992), as well as higher levels of emotional intelligence (Mikolajczak, Bodarwe, Laloyaux, Hansenne, \& Nelis, 2010) and psychological well-being (Urry et al., 2004). In this article, we specifically investigate whether frontal asymmetry can serve as a potential correlate or predictor of PTSD symptoms.

Exploring the link between frontal asymmetry, PTSD, and other mental disorders is important for several reasons. Firstly, with its 20 different symptoms, PTSD is among the most complex and heterogeneous mental disorders listed in the latest version of the Diagnostic and Statistical Manual (DSM-5; American Psychiatric Association, 2013). In line with factor-analytic findings (Friedman, Resick, Bryant, \& Brewin, 2011; Yufik \& Simms, 2010), these symptoms are grouped in four symptom clusters (i.e., intrusions, avoidance, altered mood and cognitions, altered arousal and reactivity). Evidence suggests that intrusion symptoms, including dissociative amnesia and flashback memories, are unique to PTSD (Brewin, 2011; Bryant, O’Donnell, Creamer, McFarlane, \& Silove, 2011). In contrast, PTSD likely shares a number of fear-related symptoms with other anxiety disorders, whereas other symptoms are reminiscent of depressive symptoms (Forbes et al., 2012). Yet another set of symptoms has been argued to reflect general distress symptoms, or levels of neuroticism, and are shared by a wide range of psychopathological conditions (Zoellner, Pruitt, Farach, \& Jun, 2014). As a consequence, if a reliable marker of PTSD exists, it remains to be seen what symptom cluster is predicted, and whether it can serve to differentiate between PTSD and related clinical conditions.

The second reason why the association between frontal asymmetry, PTSD, and other mental disorders is important, is because studies addressing frontal asymmetry in relation to 
psychopathology typically link their findings to the involvement of left and right hemisphere brain systems in positive affect or approach motivation, and in negative affect or withdrawal motivation, respectively (Davidson, 1998a; Heller, 1993). In line with what other authors have noted (e.g., Allen \& Kline, 2004; Davidson, 2004), our overview of the literature shows that this approach, indeed, provides a helpful conceptualization of frontal asymmetry and its relevance to psychopathology. However, we also believe that a more in-depth analysis of the neuronal mechanisms underlying frontal asymmetry is essential for a full understanding of psychological adjustment to potentially traumatic experiences. We therefore aim to sketch a theoretical framework that links potential neural mechanisms underlying frontal asymmetry to neural and psychological abnormalities in PTSD.

With these considerations in mind, the present article aims to provide a comprehensive review of how frontal asymmetry is related to PTSD. To this end, we will first review the psychological construct of frontal asymmetry in relation to affective processing and psychopathology, notably depression and anxiety disorders (see, e.g., Thibodeau, Jorgensen, \& Kim, 2006). Then, we explore neural origins of frontal asymmetry, as well as their relevance to our current neuropsychological understanding of PTSD. Next, we discuss the available empirical evidence bearing on an involvement of frontal asymmetry in PTSD. Finally, we discuss theoretical and practical implications of the asymmetry findings and conclude by highlighting promising avenues for future research.

\section{The psychological construct of frontal asymmetry}

\section{Trait and state frontal asymmetry}

Studies investigating the relation between frontal EEG asymmetry and affective processes can be divided into two categories (Coan \& Allen, 2003). The first type of study relies on hemispheric asymmetry while participants are in a resting state, and relates this asymmetry to various trait-like concepts (e.g., individual differences in emotional reactivity). The most widely used procedure to obtain resting state indices of frontal asymmetry was initially described by Tomarken, Davidson, Wheeler, and Kinney (1992). These authors derived EEG asymmetry measures from eight one-minute resting periods (four with eyes open, four with eyes closed) and observed that asymmetry scores display excellent internal consistency within one session (Tomarken, Davidson, Wheeler, \& Kinney, 1992). Test-retest reliability usually ranges from 0.5 to 0.6 over periods of 2-6 weeks (Hagemann, Hewig, Seifert, Naumann, \& Bartussek, 2005; see also Towers \& Allen, 2009). Therefore, in studies 
using this method, frontal asymmetry refers to a trait-like variable that is assumed to reflect stable individual differences in affect processing. The measure of interest in this type of study has been referred to as trait frontal asymmetry. In the second type of study, fluctuations in EEG asymmetry are investigated in relation to manipulations that intend to influence affective states (e.g., the Directed Facial Action Task; Coan, Allen, \& Harmon-Jones, 2001). The measure of interest in the latter type of study has been labelled state-dependent frontal asymmetry (e.g., Coan \& Allen, 2004).

Importantly, state-dependent frontal asymmetry and trait frontal asymmetry measured during a resting state are not independent of one another (see also Chapter 5). Phasic or statedependent frontal asymmetry is superimposed on tonic or trait-like asymmetry (Hagemann, 2004). In fact, Hagemann, Naumann, Thayer, and Bartussek (2002) performed a latent statetrait analysis of frontal asymmetry in healthy participants and estimated that 60 percent of the variance in resting EEG asymmetry can be ascribed to a stable trait, whereas 40 percent of the variation reflects state-dependent fluctuations. For studies on trait frontal asymmetry, this means that longer and repeated measurements are probably better able to capture a latent trait. In addition, Coan et al. (2006) argued that individual differences in frontal asymmetry during experimental emotion inductions are also reasonably stable and reflect meaningful individual differences. Accordingly, measures of state-dependent frontal asymmetry may even be more reliable that resting measurements, because they allow better control over state fluctuations in frontal asymmetry.

In sum, trait frontal asymmetry and state-dependent frontal asymmetry during emotion processing may yield complementary information about individual differences in emotion, psychopathology, and possibly PTSD. However, until recently (e.g., Allen \& Cohen, 2010; Coan et al., 2006; Goodman, Rietschel, Lo, Costanzo, \& Hatfield, 2013; Lopez-Duran, Nusslock, George, \& Kovacs, 2012; Stewart, Coan, Towers, \& Allen, 2014; Stewart, Coan, Towers, \& Allen, 2011), studies addressing frontal asymmetry in relation to individual differences and psychopathology exclusively followed the trait frontal asymmetry approach. In contrast, studies into the effects of state-dependent changes in frontal asymmetry have mostly focused on general principles of emotion processing (e.g., understanding frontal asymmetry conceptually) rather than on individual differences (Coan \& Allen, 2003; HarmonJones et al., 2010). In the following, we briefly summarize the findings and highlight conceptual issues. 


\section{Correlates and conceptualization of frontal asymmetry}

Motivational direction. Both relatively left-sided and right-sided frontal activation (i.e., reduced alpha power) can be induced by a variety of emotion-eliciting situations. Such phenomena have been observed in response to emotional stimuli (e.g., Gable \& HarmonJones, 2008) as well as during voluntary emotional expression (e.g., Coan et al., 2001; Ekman \& Davidson, 1993). Generally, studies converge on the conclusion that relatively left-sided frontal activation can be observed during states of heightened approach motivation such as happiness. In contrast, relatively right-sided frontal activation is typically associated with heightened withdrawal motivation such as fearful or sad emotional states.

However, effect sizes vary across studies, and there are some failed replications. Such inconsistencies might be related to methodological differences between studies (for a discussion, see Coan \& Allen, 2004; Hagemann, Naumann, \& Thayer, 2001), but could also be due to the difficulty to disentangle the critical factors in asymmetric activation. This is particularly relevant for the induction of anxiety, which might result in ambivalent directional motivation, including fear (i.e., a motivation to withdraw) and strategies of active avoidance that may involve approach-related goals and actions. Similarly, several studies have failed to find a link between frontal asymmetry and behavioural inhibition, a complex motivational state that involves the interruption of ongoing approach behaviours (e.g., Wacker, Chavanon, Leue, \& Stemmler, 2008). These conceptual difficulties might in part explain the inconsistent findings with respect to withdrawal motivation in healthy and clinical samples and relatively right-sided frontal activity, and may be particularly relevant for PTSD patients, who display both fear and avoidance symptoms.

Like state-dependent frontal asymmetry, trait frontal asymmetry has also been associated with motivational direction (for reviews, see Coan \& Allen, 2004; Harmon-Jones et al., 2010). There is abundant evidence showing that relatively left-sided frontal activity is associated with stable behavioural tendencies involving approach, appetite, or behavioural activation. For instance, social observational studies have found predominantly left-sided frontal activity to be linked to approach-related interpersonal concepts such as social competence and trait anger (for a review, see Harmon-Jones, 2004). Right-sided frontal activity has often, but less consistently, been related to stable behavioural tendencies involving avoidance, withdrawal, or behavioural inhibition (Sutton \& Davidson, 1997). The literature addressing trait frontal asymmetry includes failed replications (Hagemann et al., 
2001; Reid, Duke, \& Allen, 1998). Still, for both trait and state frontal asymmetry, there is good support for the motivational directions model of frontal asymmetry (Davidson, 1998a).

Depression. Many studies have addressed the role of frontal asymmetry in psychological dysfunctions, thus focusing on its potential clinical relevance. Despite some exceptions (e.g., Reid et al., 1998), a recurrent finding is that frontal asymmetry is associated with current and lifetime depression. That is, current or past depression has been linked to higher relatively right-sided frontal activity in a resting state (Davidson, 1998a; Heller \& Nitschke, 1998; Mathersul, Williams, Hopkinson, \& Kemp, 2008; Stewart, Bismark, Towers, Coan, \& Allen, 2010; Thibodeau et al., 2006) and to greater relatively right-sided activation during emotional challenges (Stewart et al., 2014; Stewart et al., 2011). Other studies have used resting state frontal asymmetry as a parameter that may inform about treatment effects. For example, Barnhofer and colleagues (2007) showed that mindfulness-based cognitive therapy in persons with a previous history of depression consolidates relative left frontal activity, whereas a control intervention was associated with decreased left frontal activity. Notably, a study by Moscovitch et al. (2011) found that relatively more left-sided frontal activity predicted beneficial treatment responses in a sample of patients with depression or social anxiety, who were treated with cognitive behavioural therapy.

In addition, a few studies have shown that frontal asymmetry can predict psychopathology in at-risk individuals. For instance, children and adolescents with a familial risk of depression, compared to no-risk control participants, display more relatively rightsided frontal activity at rest (Tomarken, Dichter, Garber, \& Simien, 2004) and more rightsided activation in response to happy and sad films (Lopez-Duran et al., 2012). Moreover, within such a familial risk group, more relatively left-sided frontal activation during an emotional provocation was found to dampen the association between negative life events and internalizing symptoms (Lopez-Duran et al., 2012). Likewise, frontal asymmetry has been linked prospectively to the risk of developing depression (Nusslock et al., 2011). Another study found relatively more right-sided frontal activity in individuals with extremely low birth weight (L. A. Schmidt, Miskovic, Boyle, \& Saigal, 2010), a group known to be at heightened risk to develop stress-related psychological problems. Overall, the findings suggest that both trait and state frontal asymmetries are informative individual difference variables that are especially relevant to depression.

Anxiety. There are also clear indications that frontal asymmetry is associated with vulnerabilities relevant to anxiety disorders. For instance, highly anxious-reactive children, who are at risk of developing an anxiety disorder later in life, display more right-sided frontal 
activity and less left-sided frontal activity than children with low levels of this risk factor (Kagan \& Snidman, 1999). Compared to non-anxious controls, anxious individuals have been shown to display more right-sided activation in response to induced anxiety or fear (Davidson, Marshall, Tomarken, \& Henriques, 2000). Such a fear-induced activation correlates with enhanced bias towards fear processing and with increases in negative mood (Avram, Baltes, Miclea, \& Miu, 2010). Furthermore, compared to controls, panic disorder patients were found to display more right-sided frontal activity at rest and more right-sided frontal activation in response to anxiety-relevant stimuli (Wiedemann et al., 1999). However, some studies yielded null-findings or even conflicting results, such as that higher left-sided activity correlated with trait anxiety and anxious apprehension (Heller, Nitschke, Etienne, \& Miller, 1997; for a review, see Thibodeau et al., 2006). This indicates that the study of anxiety might be particularly prone to confounding influences of different contributions to frontal asymmetry (e.g., fear vs. active avoidance), requiring it to be disentangled carefully.

The empirical link between frontal asymmetry and both depression and anxiety disorders is further underlined by a meta-analysis that included both types of patients (Thibodeau et al., 2006). Thibodeau and colleagues revealed that across studies, relatively more right-sided frontal activity at rest is linked to both depression and anxiety disorders. A smaller, similar association emerged for patients with comorbid depression and anxiety. However, a potential problem in the included studies is that subclinical or sub-threshold anxiety is difficult to disentangle from depression, and vice versa. Therefore, based on these findings, it is difficult to disentangle whether the association with frontal asymmetry is carried by depression or anxiety symptoms, by both, or by a common third variable (e.g., general distress, neuroticism). Still, the studies reviewed in this section jointly indicate high relevance of right-sided frontal activity and activation to current depression, anxiety disorders, and for the risk to develop psychopathology. Because PTSD has a considerable factorial overlap with both depression and anxiety disorders, it is reasonable to expect right-sided frontal asymmetry to be indicative of PTSD as well.

\section{Neuroanatomical links between frontal asymmetry and psychopathology}

While the above-mentioned studies suggest an intimate relationship between frontal asymmetry and psychopathology, it is worthwhile to understand this link from a neuropsychological perspective. In particular, describing asymmetrical brain processes may inform neuropsychological models of PTSD. Surprisingly, it is not yet well-understood how 
frontal asymmetry is associated with other functional brain imaging findings (Allen \& Kline, 2004; Davidson, 2004). One reason for this is that alpha band power derived from EEG recordings cannot be easily translated into regional blood flow or metabolism changes that are usually assessed in brain imaging studies (de Munck, Goncalves, Mammoliti, Heethaar, \& da Silva, 2009; Klimesch et al., 2007). Also, frontal asymmetry metrics are derived from averages of lateralized alpha power over periods of several minutes. Thus, EEG asymmetry studies differ in terms of temporal specificity from most neuroimaging studies that aim to localize neuronal activity within more narrow time windows (Cohen, 2011). Finally, relatively few neuroimaging studies have explicitly addressed lateralization effects in their statistical analyses. Rather, they have usually performed separate correlation or group analyses for structures in each hemisphere (i.e., simple effects), without explicitly testing the interaction of the effects with hemisphere (for discussion, see Davidson \& Irwin, 1999; Spielberg, Heller, \& Miller, 2013).

Still, at least two potential neuroanatomical underpinnings of frontal asymmetry have been discussed in the literature (Davidson, 1998a; Pizzagalli, Sherwood, Henriques, \& Davidson, 2005). The first is based on the observation that the largest contribution to frontal asymmetry measured with scalp electrodes may originate from the dorsolateral prefrontal cortex (dlPFC; Pizzagalli et al., 2005). Although the role of left and right dlPFC in emotional valence or motivational direction is still debated (for meta-analyses, see Murphy, NimmoSmith, \& Lawrence, 2003; Wager, Phan, Liberzon, \& Taylor, 2003), recent neuroimaging studies corroborate the view that the dlPFC is involved in frontal asymmetry, whereby the left and right dlPFC sustain approach and avoidance actions, respectively (Berkman \& Lieberman, 2010; Herrington et al., 2010). Thus, differential roles of the left and right dlPFC might be of interest for the involvement of frontal asymmetry in affective disorders.

The second association between frontal asymmetry and neuroanatomical models is based on speculations about the neural correlates of lateralized approach and withdrawal systems within and beyond the PFC (e.g., Davidson, 1998a, 1998b). Of particular interest here are lateralizations in widespread neuronal systems, prominently featuring the ventromedial PFC (vmPFC) and the amygdala, that may contribute to frontal asymmetry and that are involved in various emotional disorders. Therefore, in the following paragraphs, we elaborate on the potential involvement of the dlPFC, amygdala, vmPFC and other lateralized brain systems in frontal asymmetry and discuss their potential relevance for understanding psychopathology in general, and PTSD in particular. Figure 6.1 displays the location of several key regions in the 
brain (panel a) and a schematic representation of their potential roles in PTSD that are elaborated in the following paragraphs.

\section{Possible roles of the left and right dIPFC}

Cognitive emotion regulation. Functional magnetic resonance imaging (fMRI) studies suggest that the dIPFC mediates cognitive aspects of emotion regulation. For instance, the lateral PFC is implicated in cognitive reappraisal (Drabant, McRae, Manuck, Hariri, \& Gross, 2009; Ochsner \& Gross, 2005; but see Kompus, Hugdahl, Öhman, Marklund, \& Nyberg, 2009), an emotion regulation strategy that aims to alter emotional valence by changing interpretations of a situation (Gross \& Thompson, 2007). Also, cognitive modification of implicit emotional processes, such as the allocation of attention to threatening stimuli, has been shown to be associated with lateral PFC activation (Browning, Holmes, Murphy, Goodwin, \& Harmer, 2010).

Regarding lateralization, reappraisal strategies might primarily rely on the left PFC, although this could be moderated by the goal (e.g., increase or decrease emotions) and content (e.g., negative or positive emotion) of the reappraisal task (Ochsner \& Gross, 2005). For instance, one study found primarily left-sided PFC activation when participants dampened their negative emotional reactions (Ochsner, Bunge, Gross, \& Gabrieli, 2002), whereas another study found more right-sided PFC activation in participants dampened their reactions to erotic stimuli (Beauregard, Levesque, \& Bourgouin, 2001). Notably, the first study also involved a more verbal reappraisal strategy than the second. Thus, left-sided frontal activation might reflect reappraisal with a hedonic goal, as well as recruitment of lateralized structures involved in verbal and non-verbal stimulus processing (Ochsner et al., 2002). Importantly, these findings still await replication and a direct statistical test of lateralization. Yet, a recent EEG study supports the association between left-frontal activation and reappraisal. In particular, Parvaz, MacNamara, Goldstein, and Hajcak (2012) showed that cognitive reappraisal during positive and negative picture viewing was associated with reduced eventrelated alpha power (which could be interpreted as increased activation) over the left forehead. Thus, state-dependent frontal asymmetry may be indicative of engagement in cognitive reappraisal by reflecting the differential recruitment of the left and right dlPFC. 
a)

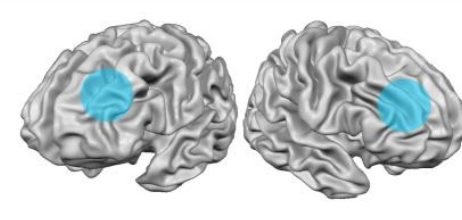

dIPFC

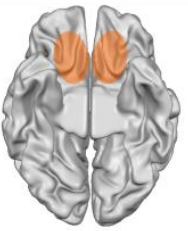

vmPFC

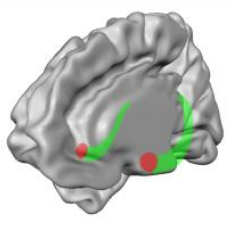

Hippocampus (green) Amygdala (red)

b)

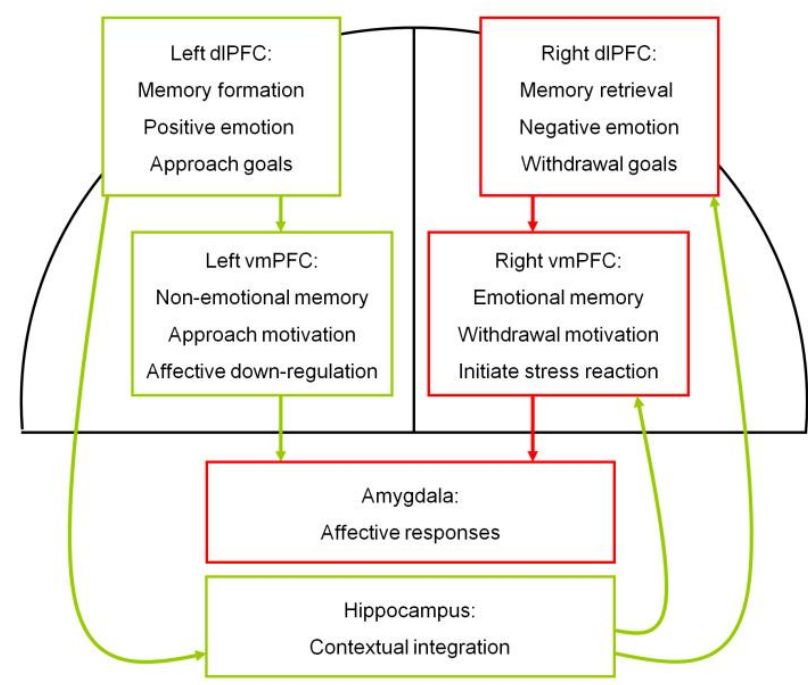

c)

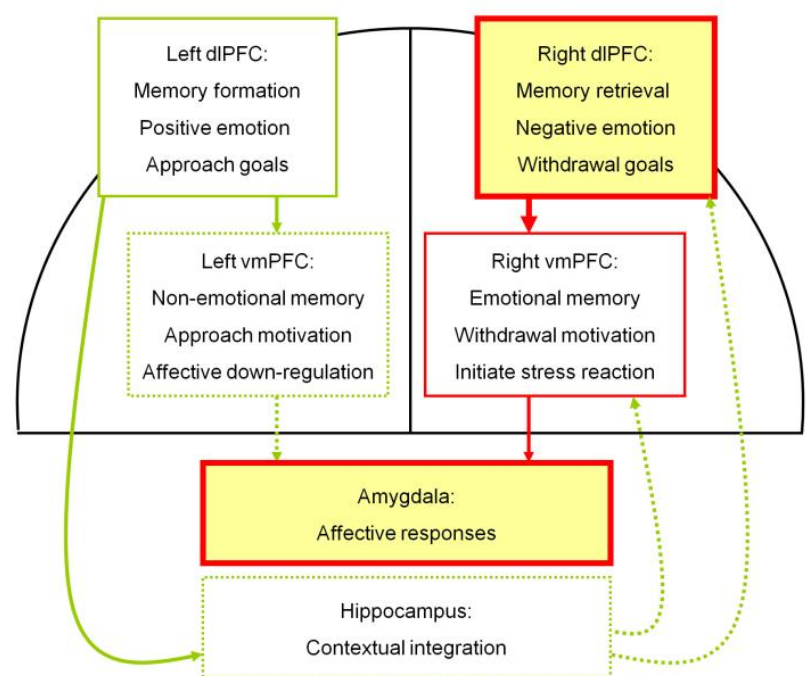

Figure 6.1. A schematic model of hemispheric preference in the frontal cortex and pathways to the amygdala and hippocampus relevant for adapting to trauma (in green), and involved in PTSD symptoms (red). a) Location of the involved brain areas; b) normal functioning; c) pathological functioning as seen in PTSD, with degraded activity and pathways involving left vmPFC and hippocampus (dashed lines) and hyper-activity in amygdala and right dlPFC (heavier lines) due insufficient contextual integration. Note that for clarity, differences between left and right structures are emphasized, but they typically overlap functionally. Also, the selective focus on functions, regions, asymmetries, and one-directional pathways necessarily omits others that can be of interest. 
Memory. With respect to dIPFC asymmetries, other neuroimaging findings suggest that the left and right dlPFC may have differential roles in memory processes. In particular, during formation of a memory trace for simple verbal or visual stimuli, the left dlPFC is preferentially activated as compared to the right dIPFC, whereas the opposite pattern has been observed during retrieval of such a memory (Habib, Nyberg, \& Tulving, 2003; Henson, Rugg, Shallice, \& Dolan, 2000). The right PFC appears to reflect specific monitoring processes that occur directly after the retrieval of recently acquired memories (for review, see Gilboa, 2004) and is recruited especially in more difficult recall tasks (Fletcher \& Henson, 2001; Henson et al., 2000) or when recognition depends on contextual information (King, Hartley, Spiers, Maguire, \& Burgess, 2005).

Only few studies have addressed the direct relationship between frontal EEG asymmetry and the encoding or retrieval phases in memory tasks (Nitschke, Heller, Etienne, \& Miller, 2004) (see also Babiloni et al., 2004). These do not support the view that frontal asymmetry is directly involved in (trauma) memory encoding or retrieval. Rather, Nitschke et al. (2004) found effects of frontal asymmetry on memory performance that interacted with levels of depression. In particular, only in individuals with low depression scores, left-sided frontal activation (i.e., reduced alpha activity) during a preparatory phase preceding a sad narrative predicted better recognition of that narrative. For individuals scoring high on depression, more right-sided activation during the encoding phase of the sad narrative predicted better recognition performance. Nitschke et al. point out that these findings do not necessarily reflect an asymmetric encoding and retrieval processes. Group differences in approach-related encoding strategies, or in a negative information processing bias might as well account for their findings. Note, however, that the available studies are limited to recognition performance (Babiloni et al., 2004; Nitschke et al., 2004). In sum, despite the prominent role of memory phenomena in PTSD, the role of frontal asymmetry in narrative memory, involuntary memory, or PTSD flashbacks still remains to be investigated. As well, studies still need to show whether the asymmetric recruitment of the dlPFC during encoding and retrieval mediates adaptive and/or maladaptive responses to trauma.

Transcranial magnetic stimulation studies. The involvement of the left and right dlPFC in emotion and memory has been further documented in studies using repetitive transcranial magnetic stimulation (rTMS). rTMS can be used to interfere with cortical activity using strong and repetitive magnetic pulses, whereby different pulse frequencies can either reduce (low frequencies) or increase (high frequencies) the neuronal excitability of a target cortical region. With this technique, researchers have shown that enhanced right, but not left, dlPFC 
excitability leads to impaired inhibition of negative emotional information (Leyman, De Raedt, Vanderhasselt, \& Baeken, 2009). Increased left dlPFC excitability was found to enhance the processing of positive emotional information while additionally attenuating insula responses to negative emotional information (Baeken, Van Schuerbeek, et al., 2011). Also, there are indications that when right dlPFC excitability is enhanced, more stress hormones are released when state anxiety levels are high (Baeken, Vanderhasselt, \& De Raedt, 2011).

Concerning memory processes, recent rTMS studies appear to correspond with the view that the left dlPFC is crucial for encoding or consolidation of new memories, whereas the right dIPFC is crucial for memory retrieval. For instance, recognition memory for pictures and words is impaired when TMS interferes with the left dlPFC during post-perceptual processing of to-be-learned pictures (i.e., $300 \mathrm{~ms}$ after picture onset; Rossi et al., 2011). Likewise, rTMS stimulation of the right dlPFC following encoding (Turriziani et al., 2012) or following cued memory reactivation (Sandrini, Censor, Mishoe, \& Cohen, 2013) can enhance memory performance, whereas stimulation of that site during retrieval can impair performance (Sandrini, Cappa, Rossi, Rossini, \& Miniussi, 2003). However, further replications are warranted, as the few existing studies diverge on several parameters, including the TMS protocols, time of stimulation, and targeted memory phases. Also, more studies are needed to identify potential moderators in the encoding-retrieval asymmetry, including verbalization (see Epstein, Sekino, Yamaguchi, Kamiya, \& Ueno, 2002), retrieval strategies (see Manenti, Cotelli, Calabria, Maioli, \& Miniussi, 2010), or retrieval facilitation (see Sandrini et al., 2003).

Taken together, fMRI and rTMS studies seem to support the view that the left and right dlPFC are differentially involved in cognitive emotion regulation, emotional processing, and in memory processes. In particular, more left-sided activity might be associated with positive emotion, down-regulation of negative emotion, and memory encoding, as opposed to negative emotion, down-regulation of positive emotion, and memory retrieval. The emotion- and memory-related asymmetries could be additive or interact with each other (see, e.g. Weigand et al., 2013). Accordingly, relatively left-sided dIPFC activity would be expected to have favourable consequences for coping with trauma. 


\section{Possible Roles for Left and Right Medial PFC and Amygdala}

Importantly, the above-mentioned rTMS studies did not only locally manipulate the dIPFC but likely also had more distal effects, for example on ventral prefrontal and limbic areas (e.g., Teneback et al., 1999). This supports the notion of left and right dIPFC as parts of a larger neural network that is commonly activated during a variety of cognitively demanding situations, and that has been suggested to generally support adaptive goal-directed behaviour (Duncan \& Owen, 2000; Niendam et al., 2012). This network is believed to be functionally organized along a medial-lateral axis, with emotional processing mainly involving medial frontal structures and cognitive processing mainly involving lateral frontal structures (for review, see Phillips, Ladouceur, \& Drevets, 2008). While the dlPFC is often regarded as a network that sustains cognitive functions (e.g., maintaining information in working memory; C. E. Curtis \& D'Esposito, 2003), brain imaging studies show that processing of emotional valence essentially involves the medial prefrontal structures (e.g., Heinzel et al., 2005; for a meta-analysis, see Kober et al., 2008) (but see S. Grimm et al., 2006; Viinikainen et al., 2010). Likewise, processing as well as regulation of emotional (e.g., Depue, Curran, \& Banich, 2007; Kompus et al., 2009) and self-relevant information (Gilboa, 2004; Northoff et al., 2006) have typically been associated with medial prefrontal activation, such as within the vmPFC. The medial and lateral systems processing emotion and cognition may have a close and competitive functional relationship (e.g., Vytal, Cornwell, Arkin, \& Grillon, 2012; Yun, Krystal, \& Mathalon, 2010), and some researchers have speculated that emotional disorders may be characterized by an imbalance between them (Northoff et al., 2004). Thus, the relevance of a larger prefrontal network beyond the dIPFC should be taken into account when exploring the neural origins of frontal asymmetry.

Davidson (1998a, 2000) proposed that important contributions to frontal asymmetry might originate from activations within a larger network including the temporal polar regions, amygdale, basal ganglia, and hippocampi. Accordingly, within such a network, approach and withdrawal motivations are represented in the lateral PFC as goal states held in working memory. These goals are transferred to medial prefrontal structures that keep behaviouralreinforcement contingencies in working memory. Approach-related information is further transmitted to the nucleus accumbens and to the basal ganglia in order to coordinate and execute action plans. In contrast, goals related to withdrawal motivation might critically involve the amygdala. 
Indeed, there appear to be several lateralized regions within the medial PFC that are consistently activated during manipulations of motivational direction (Wager et al., 2003). In line with the motivational direction model of frontal asymmetry, asymmetric activation within the medial PFC during cognitive task performance has more recently been found to predict trait motivational direction (Spielberg et al., 2011). Furthermore, animal studies suggest that autonomic and neuroendocrine reactions to stress predominantly implicate the right vmPFC (Cerqueira, Almeida, \& Sousa, 2008; Sullivan \& Gratton, 2002). In particular, the right vmPFC appears to be responsible for hormonal stress responses, stress ulcer formation, and stress-induced deterioration of the connection between the frontal cortex and the hippocampus (Cerqueira et al., 2008; Cerqueira, Mailliet, Almeida, Jay, \& Sousa, 2007).

In humans, direct tests of lateralization in the vmPFC and amygdala are still warranted. Yet, preliminary evidence suggests more right-sided medial PFC activation during the processing of emotional autobiographical memories, compared to non-emotional memory processing (Cabeza \& St Jacques, 2007; Svoboda, McKinnon, \& Levine, 2006). Moreover, right vmPFC activation during negative memory retrieval was found to correlate with amygdala activity (Greenberg et al., 2005). Data also suggests that the left and right amygdale respond differently during specific emotional states. For instance, a positron emission tomography (PET) study using emotionally provocative films (L. Kilpatrick \& Cahill, 2003) found that enhanced right amygdala activation correlated with enhanced memory of the films, possibly because it modulates activity in the right parahippocampal gyrus and vmPFC. Other studies suggest that the right amygdala is preferentially responsive during automatic, compared to controlled, emotional states (e.g., Dyck et al., 2011; although see McMenamin \& Marsolek, 2013). It has been speculated that such enhanced right amygdala activity might be directly responsible for right-sided alpha de-synchronization and could, therefore, influence measurements of frontal asymmetry (Kagan \& Snidman, 1999). In sum, enhanced right-sided activity in the vmPFC and in the amygdala might reflect increased processing of negative information and the automatic initiation of stress responses.

The view that left-sided activity in both vmPFC and the basal ganglia accounts for the association between left-sided frontal activity and approach motivations has been supported in recent fMRI studies (Spielberg et al., 2011; Spielberg et al., 2012). Therefore, it may well be the case that both right-lateralized vmPFC and amygdala activity, and left-sided activity in the vmPFC and the basal ganglia contribute to scalp recordings of frontal asymmetry. Together, this might account for the associations between frontal asymmetry and stable or transient withdrawal and approach motivations. Of course, this interpretation remains speculative, 
because EEG recordings mostly reflect cortical activity, and lateralization in sub-cortical brain areas might not or even be reversely linked to frontal asymmetry as measured with EEG (for discussion, see e.g., Ahern et al., 2001).

\section{Non-Specific Hemispheric Biases}

Frontal asymmetry might also reflect a global and unspecific bias towards any cognitive function that is preferentially performed by one of the two cerebral hemispheres. In fact, there appear to be distinct cognitive mechanisms that are lateralized independently of each other in the brain and that might be affected by activation asymmetries. In particular, a resting-state fMRI study identified left-lateralized networks involved in internal thoughts and language processes, and right-lateralized networks involved in vision and attention (Liu, Stufflebeam, Sepulcre, Hedden, \& Buckner, 2009). Therefore, frontal asymmetry might be related in a nonspecific way to information processing biases. This view is supported by evidence of a strong interrelation between different and seemingly unrelated motor, cognitive, and emotional functions that are thought to display preferential processing in one hemisphere. For instance, activation of the left motor cortex through hand contractions (i.e., squeezing a ball) was found to increase left frontal activity and approach-related affective states (Harmon-Jones, 2006). The other way round, transient inductions of approach motivations have been shown to facilitate cognitive performances thought to depend on the left hemisphere, whereas withdrawal motivations and emotional arousal facilitate cognitive performances that depend on the right hemisphere (Papousek, Schulter, \& Lang, 2009; Simon-Thomas, Role, \& Knight, 2005). Additionally, animal studies suggest an association between leftward turning behaviour and left paw preference (i.e., right-hemisphere preference of motor functions) on the one hand, and stronger hormonal responding in stress inductions, on the other (Sullivan \& Gratton, 2002).

It is not yet fully understood why seemingly unrelated functions displaying a hemispheric preference would mutually influence each other. One plausible explanation is that hemispheric lateralization of functions follows a principle of human bodily organization, which allows for efficient use of energy. For instance, there is evidence for hemispheric lateralization in the cerebral control of the autonomic nervous system (ANS; e.g., Foster \& Harrison, 2006). Some authors have proposed that the left and right forebrain may serve functions of energy enrichment and energy expenditure, respectively (Craig, 2005). Despite possible anomalies and complications (for a review, see Hagemann, Waldstein, \& Thayer, 
2003), this view is supported by evidence of right hemisphere dominance in sympathetic function and left hemisphere dominance in parasympathetic function (e.g., Wittling, Block, Genzel, \& Schweiger, 1998), or evidence suggesting a general right-hemisphere dominance in the cerebral mediation of ANS functions (e.g., Ahern et al., 2001; Johnsen \& Hugdahl, 1993). Thus, various neuropsychological functions predominantly sustained by either hemisphere may be closely related due to their common involvement in cerebral control of the ANS. With this in mind, one could argue that frontal asymmetry might capture a global preference towards a set of neuropsychological functions that are linked through a functional principle of the brain's architecture.

Following this line of reasoning, relatively more right frontal activity could be expected to facilitate visual processing (particularly of negative emotional information; see Kensinger \& Choi, 2009) as well as processing of specific stimulus information (see, e.g., Marsolek, 1999; Marsolek \& Burgund, 2008). Also, a preference for right hemisphere functioning might dampen the formation of meaningful semantic connections (see, e.g., Dolan \& Fletcher, 1997; Fletcher, Shallice, \& Dolan, 1998), verbal processing (Fletcher \& Henson, 2001), and abstract information processing (Marsolek, 1999; Marsolek \& Burgund, 2008). According to clinical theories of PTSD (Brewin, Gregory, Lipton, \& Burgess, 2010; Ehlers, 2010), such a pattern of preferential processing (e.g., favouring data-driven processing over conceptual processing) may have deleterious effects during aversive experiences such as more intrusive memories (e.g., Kindt, van den Hout, Arntz, \& Drost, 2008; see also Chapter 2). Thus, frontal asymmetry could have indirect effects on the development of specific PTSD symptoms through a broader and unspecific bias towards hemispheric processing.

Notably, the potential importance of hemispheric preferences in emotional processing has been discussed for decades. For example, Tucker and Newman (1981) proposed that a preference of functions sustained by the left hemisphere would enhance the ability to inhibit emotional reactivity. In particular, through stronger reliance on verbal and analytic processing, individuals with a "left hemisphere preference" would inhibit emotional arousal more efficiently by using abstraction and disengagement (but see Merckelbach, Dijkstra, De Jong, \& Muris, 1994). These hypotheses have also been tested using the Preference Test (PT; Zenhausern, Notaro, Grosso, \& Schiano, 1981), a global self-reported scale assessing subjective preference of cognitive and behavioural processes that are assumed to rely on either of the hemispheres. De Jong, Merckelbach, and Nijman (1995) found that right hemisphere preference on the PT predicted depression and anxiety levels, although it was unrelated to an index of cognitive bias that was assumed to underlie the maintenance of 
anxiety. However, the relationship between hypothesized thinking styles and actual hemispheric asymmetry remains somewhat inferential, because PT scores correlate only moderately $(r=.31)$ with frontal asymmetry scores (Merckelbach, Muris, Pool, De Jong, \& Schouten, 1996). Still, these findings provide limited support for the idea that global hemispheric thinking styles are related to psychopathology.

A noteworthy alternative proposition concerning hemispheric processing has been put forward by Weinberg (2000) and later on by Rotenberg (2004). Both argue that the predominant processing modes of the two hemispheres differ essentially in terms of how information is organized contextually. In particular, left hemisphere structures would preferably embed information in a non-ambiguous and mono-semantic context, which allows formal, logical, and probabilistic thinking. Structures in the right hemisphere would, in contrast, preferably embed information in an integral, poly-semantic, and ambiguous context, giving rise to intuitive and creative thinking. Based on observations suggesting that early trauma disrupts the development of the right limbic system (for a review, see Schore, 2002), these authors propose that qualitative disruption of right-hemisphere functionality may cause depression (Rotenberg, 2004) and lead to suicidality (Weinberg, 2000). Thus, rather than associating left hemisphere functions with adaptive, and right hemisphere functions with maladaptive outcomes, this view contends that deficiencies in the right hemisphere can induce a maladaptive shift towards functions sustained predominantly in the left hemisphere, which would result in inflexible problem solving, cognitive rigidity, dissociation, and impaired affect regulation.

Importantly, Weinberg (2000) proposes that right hemisphere activation (i.e., decreased alpha activity) is indicative of a right hemisphere deficiency. This seems to contradict widely held and more recent interpretations of alpha either as a straightforward index of cortical idling (Pfurtscheller et al., 1996), or as a consequence of pulsed inhibition (Jensen, Bonnefond, \& VanRullen, 2012), which locally reduces functional connectivity (Scheeringa, Petersson, Kleinschmidt, Jensen, \& Bastiaansen, 2012). However, Weinberg's proposal can be reconciled with other conceptualizations of alpha activity (e.g., Buzsaki \& Draguhn, 2004; Klimesch et al., 2007) as a selective top-down process that protects information processing against interference. In sum, traditional models of global hemispheric preference can be reconciled with the idea that relatively more left-sided activity protects against emotional challenges, whereas heightened relative right-sided activity reflects potentially maladaptive processing. 


\section{Linking the neurocircuitry of PTSD to frontal asymmetry}

\section{Neuroimaging findings in PTSD}

Neuroimaging research on PTSD has focused most intensively on the amygdala, the hippocampus, and the prefrontal cortex (Francati, Vermetten, \& Bremner, 2007; McNally, 2006; Shin, Rauch, \& Pitman, 2006). In their review of the extant literature, Shin and colleagues (2006) concluded that altered functioning of these brain regions is a relatively reliable finding in PTSD (c.f. Francati et al., 2007; Patel, Spreng, Shin, \& Girard, 2012). In particular, PTSD patients typically display more amygdala activity in response to traumarelevant stimuli than controls, whereas activity in the medial PFC is reduced. Meanwhile, a volumetric reduction of PTSD patients' hippocampi has often been found, along with excessive activation in these structures across a wide range of tasks (Patel et al., 2012). This pattern of neuronal responding might distinguish PTSD from other anxiety disorders (see also Etkin \& Wager, 2007) and appears to be in part a predisposing factor for the development of PTSD and in part a consequence of traumatic experiences (van Wingen, Geuze, Vermetten, \& Fernandez, 2011).

The functional significance of the above-mentioned neuroimaging findings can be explained by considering the vmPFC and the amygdala as a core affect network that regulates emotional responses. By default, the amygdala responds to emotionally salient stimuli, but is inhibited by the vmPFC in a context-dependent manner (Suvak \& Barrett, 2011; Thayer, Ahs, Fredrikson, Sollers, \& Wager, 2012). The vmPFC is regarded as a key structure for integrating internal and external representations of the situational context, autobiographical memory, emotional appraisals, and the regulation of behavioural and physiological responses (Svoboda et al., 2006; Thayer et al., 2012). The inhibitory control of the vmPFC on the amygdala therefore depends on memory and cognitive appraisals (e.g., the experience of cognitive control; Maier \& Watkins, 2010). In PTSD, the reduced vmPFC activity has been proposed to reflect decreased control over bodily responses, which disinhibits the default amygdala responses to threat and thereby produces states of hypervigilance (Suvak \& Barrett, 2011; Thayer et al., 2012). This mechanism would also underlie the specific sensitization of the amygdala to trauma-related stimuli (Francati et al., 2007).

This hypothesis is supported by recent findings in police officers showing that both resilience to traumatic experiences and better recovery from PTSD are marked by enhanced vmPFC and reduced amygdala activity during trauma memory retrieval (Peres et al., 2011). However, some conflicting evidence exists. That is, during negative (not traumatic) memory 
retrieval, PTSD patients were found to display exaggerated vmPFC activity that was coupled with higher amygdala responsivity (St Jacques, Botzung, Miles, \& Rubin, 2011). Also, vmPFC damage can reduce, rather than increase, the risk of developing PTSD (for a review, see Koenigs \& Grafman, 2009). This suggests a crucial importance of moderating factors in the relationship of the vmPFC and the amygdala, such as contextual memory. For instance, the functionality of the vmPFC may depend on appropriate input from the hippocampus, which also shows abnormal responsivity in patients with PTSD. While the vmPFC appears to play a key role in PTSD through its involvement in a memory-guided core affect network, other PFC subregions may also be involved in PTSD through their involvement in neuronal networks for executive attention, language, and for embedding traumatic memories meaningfully with other memories (Suvak \& Barrett, 2011).

The functional abnormalities seen in PTSD patients might be used as a starting point in exploring potential involvement of frontal asymmetry in this disorder. Therefore, the next section reviews brain imaging data that are pertinent to brain asymmetries in PTSD patients. In line with our review on the potential neural origins of frontal EEG asymmetry, a particular focus is on asymmetries in the dlPFC, vmPFC, and the amygdala.

\section{Asymmetries in the Neurocircuitry of PTSD}

dIPFC. Considering the left and right dIPFC as a major source of frontal asymmetry, one might argue that these regions exert differential effects on the vmPFC via their involvement in cognitive emotion regulation (Ochsner et al., 2002). According to that view, the inability of the vmPFC to inhibit the amygdala in PTSD could in part be a down-stream effect of inefficient cognitive control by the dlPFC.

To date, studies have not yet directly compared left and right dlPFC involvement in PTSD. However, a few studies using working memory tasks reported simple effects of aberrant left, right, or bilateral dlFPC activities in PTSD. For instance, Fani et al. (2012) found that PTSD patients display enhanced left dIPFC activation when confronted with threatrelated distractors during a selective attention task, compared to conditions involving neutral or positive distracters. Moreover, this exaggerated activation correlated positively with PTSD symptoms. Another fMRI study found PTSD patients to display lower right dlPFC activation than healthy and trauma-exposed controls in an inhibitory control task with neutral stimuli (Falconer et al., 2008). Here, frontal activation inversely correlated with PTSD symptoms. Meanwhile, a PET study by Clark et al. (2003) found that PTSD patients had deficient 
activation of the left dlPFC compared to controls during a task requiring working memory updating. These findings coincide with the findings of Dolcos et al. (2013), who showed that the left dIPFC is susceptible to emotional distraction and might mediate impairments in taskrelevant executive control. Finally, other findings indicate that PTSD patients display bilateral dlPFC hyperactivity associated with the maintenance of verbal stimuli in working memory (Moores et al., 2008). Together, these results indicate that PTSD is marked by inefficient dIPFC functioning, such that emotional distractors lead to an excessive deployment of cognitive resources, which, in turn, impairs efficient processing of neutral stimuli in working memory. Regarding hemispheric asymmetry, the available data in PTSD patients suggest that especially the left dlPFC might be sensitive to disruption by emotional stimuli. However, studies explicitly testing laterality are still required, and it remains unclear how these asymmetries may translate to trait and state frontal EEG asymmetry.

More speculatively, deficient processing in the dIPFC can also be expected to affect the interaction between cognition and emotion in PTSD patients, wherein the left and in the right dlPFC may play differential roles (cf. supra, section Possible roles of the left and right $d l P F C$ ). In particular, impairments of left PFC functioning might limit the individual's ability to dampen negative emotional reactions through cognitive reappraisal (Ochsner et al., 2002). Likewise, asymmetric dlPFC deficits could have consequences for memory processes in PTSD, with a bias towards right-sided dIPFC activity facilitating retrieval of traumatic memories and inhibiting the encoding of new information. Arguably, this might contribute to a recurrent preoccupation with traumatic memories as seen in PTSD.

In sum, the available evidence suggests that particularly left dlPFC functioning might be compromised in PTSD patients, but the precise mechanisms by which dIPFC asymmetries are related to PTSD symptoms still await empirical scrutiny. The importance of further investigating these brain regions is pointed out by accumulating evidence linking dlPFC asymmetries to emotional disorders. For instance, using fMRI, abnormal asymmetric activations of the dlPFC have been found in depressed patients during an emotional judgment task (S. Grimm et al., 2008). In this study, patients showed hyperactivity in the right dlPFC that correlated with depression severity, and hypo-activity in the left dlPFC. Moreover, decreasing right dlPFC excitability with rTMS has been found to boost therapy effects for depression (Chen et al., 2013) and PTSD (e.g., Osuch et al., 2009; for a review, see Pallanti \& Bernardi, 2009; see also Tillman et al., 2011).

vmPFC and amygdala. Besides the dlPFC, there are also indications that asymmetric vmPFC and amygdala dysfunctions are present in PTSD, and that these could be related to 
frontal asymmetry. Some, but not all symptom provocation studies with PTSD patients found enhanced right-sided activation of the amygdala (for a review, see Francati et al., 2007). This activation might reflect enhanced encoding of negative emotional memories through modulation of the right parahippocampal gyrus and vmPFC (L. Kilpatrick \& Cahill, 2003). In line with this view, the right parahippocampus has frequently been found to be hyperactivated in PTSD (Patel et al., 2012). Moreover, one study found resting state functional connectivity patterns of the right amygdala with the posterior cingulate and the perigenual anterior cingulate cortices to be associated with PTSD symptoms (Lanius et al., 2010). These latter areas are thought to be involved in various functions such as monitoring sensory input, self-referential processing, and the conscious experience of emotion (Bluhm et al., 2009; Vogt, Finch, \& Olson, 1992). Also, as mentioned earlier, PTSD may be marked by exaggerated functional coupling between the amygdala and the vmPFC during retrieval of negative emotional memories (St Jacques et al., 2011). Furthermore, the right vmPFC is especially implicated in the processing of emotional autobiographical memory, contextual integration, and the control of ANS responses to stress. Thus, heightened right-sided activity in the vmPFC, reflected in lower right-sided frontal alpha activity, might make individuals more vulnerable to the negative effects of stress and undermine their stress resilience. Similarly, a more efficient right vmPFC, which might be reflected in higher right-sided alpha, would be expected to correlate with better contextual integration of trauma memories and with healthier ANS responding to stress.

Left and right hemisphere. Additionally, research indicates that PTSD patients could suffer from more global functional abnormalities of the hemispheres. For instance, PTSD patients show a preference towards more global visual processing and impaired local processing (Vasterling, Duke, Tomlin, Lowery, \& Kaplan, 2004), which is indicative of preferential right hemisphere processing. Moreover, more PTSD patients than controls show reduced left-sided cerebral specialization for fine motor functions (i.e., right-handedness; Spivak, Segal, Mester, \& Weizman, 1998). Left hand preference (i.e., right-hemisphere dominance) was found to be statistically associated with hyper-arousal symptoms in PTSD patients (Choudhary \& O'Carroll, 2007). Whereas these studies suggest a relationship between PTSD and a global preference for right-hemispheric functions, there are also indications that the right hemisphere may work less efficiently in PTSD patients. That is, PTSD appears to be characterized by deficient processing of incoming stimuli in the right hemisphere as evidenced in a dichotic listening task (Asbjornsen, 2011), and the left hemisphere might be involved in compensating for this deficit (Gerhards, Yehuda, Shoham, \& 
Hellhammer, 1997). Thus, there is evidence to suggest that PTSD is related to a heightened reliance on right-hemisphere functioning (c.f., Tucker \& Newman, 1981) and that PTSD is marked by a functional deficit in the right hemisphere (c.f., Weinberg, 2000).

Table 6.1 provides a summary of the above-mentioned considerations, while Figure 1 displays the principal brain regions, their relevant functions, as well as hypothetical pathways that may contribute to PTSD symptoms. As reviewed above, frontal asymmetry might be related to individual differences in cognitive emotion regulation and memory-relevant processes mediated by the dIPFC. As well, it could reflect individual differences in emotional responding and biased memory formation mediated by the vmPFC and the amygdala. Together, it appears plausible that frontal asymmetry partly reflects the efficiency of the PFC to regulate the amygdala in a memory-guided and context-dependent manner. The hippocampus is a key structure for the contextual integration of emotional memory, and its pathways to the right vmPFC and dIPFC may be essential for the inhibition of withdrawal and stress responses. When these connections function poorly (see Figure 1, panel c), this may increase the risk of developing PTSD symptoms. Additionally, an indirect involvement of frontal asymmetry in resilience and PTSD might occur through information processing biases mediated by various functions with lateral specialization in one of the two hemispheres. Thus, frontal asymmetry may reflect individual differences in neural networks and psychological mechanisms that are involved in the complex causation of PTSD. 


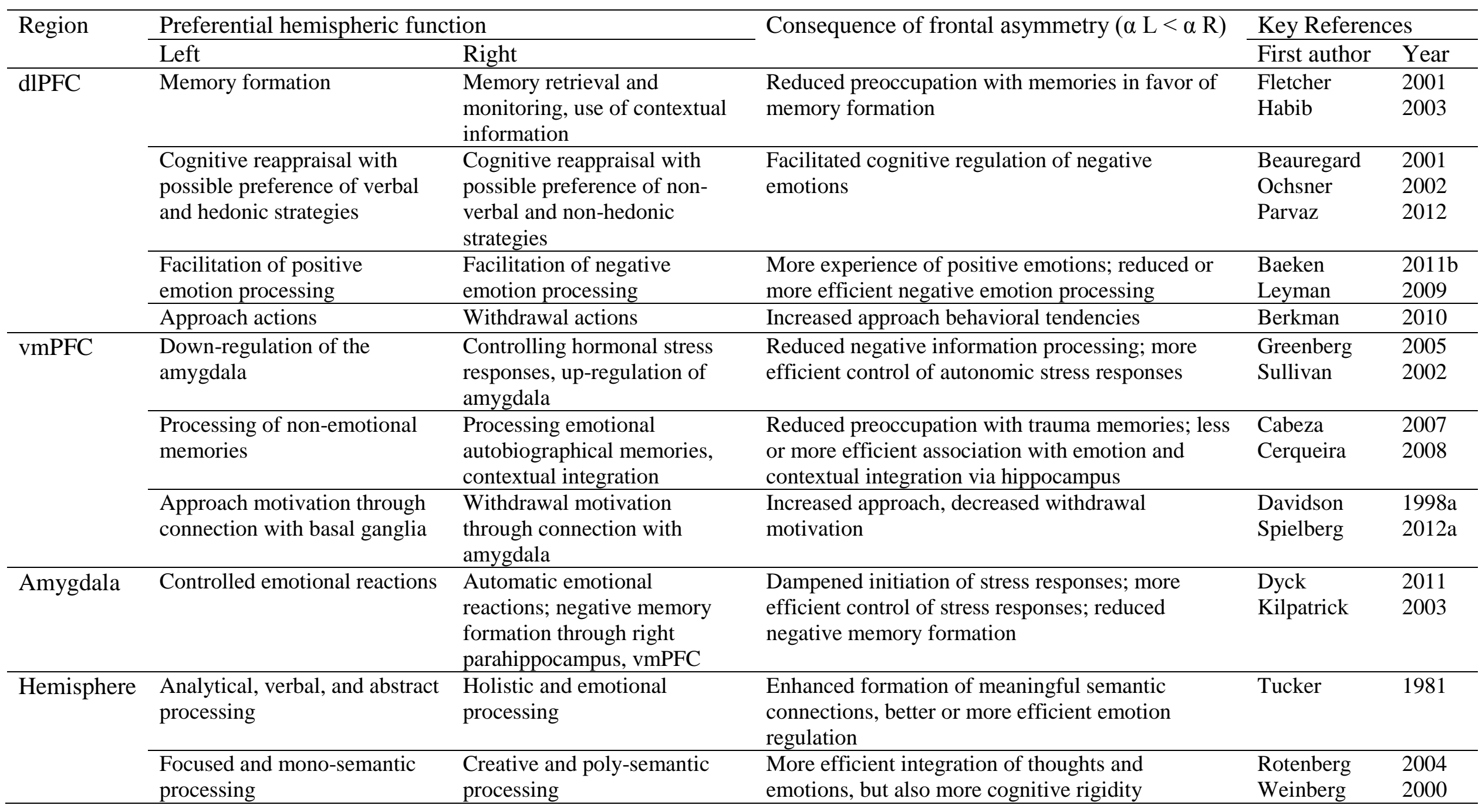

Table 6.1. Summary of Brain Processes Possibly Contributing to Frontal Asymmetry and PTSD Symptoms. $\alpha$ L $=$ alpha power over the left frontal cortex; $\alpha \mathrm{R}=$ alpha power over the right frontal cortex. 


\section{Review of frontal EEG asymmetry and PTSD}

\section{Objectives}

In the previous sections, we reviewed evidence that lends support to the idea of frontal asymmetry as a correlate of PTSD. We here provide the first systematic summary of empirical studies germane to this relationship, thereby adding to previous reviews on frontal asymmetry (e.g., Coan \& Allen, 2004; Harmon-Jones et al., 2010) and more specifically, reviews on the relation between frontal asymmetry and mental disorders (e.g., Thibodeau et al., 2006). Our objective is to determine (1) whether PTSD is marked by relatively more right-sided frontal EEG activity when compared to groups of more resilient individuals, and (2) whether frontal asymmetry can be linked directly to the severity of PTSD symptoms. Given that methodological differences between studies may account for inconsistencies (Coan \& Allen, 2003; Hagemann, 2004; Reid et al., 1998), we first provide an overview of methodological aspects that deserve special attention.

\section{Methodological issues in frontal asymmetry research}

Length and number of EEG recordings. The length of EEG recording has been shown to be a crucial factor in the reliability of the frontal asymmetry measures. That is, the test-retest reliability of trait frontal asymmetry within a session has been shown to be good with four minutes, and excellent with eight minutes of measurement (for a review, see Hagemann, 2004). State fluctuation can be reduced substantially by aggregating data from two or three measurement occasions (Hagemann, 2004). One would therefore expect that studies with longer recording periods and more measurement occasions yield more reliable findings.

Reference scheme. Studies of frontal asymmetry have employed different reference schemes for EEG measurement, with little evidence favoring any one of them as superior (Coan \& Allen, 2004; Hagemann et al., 2001; Harmon-Jones et al., 2010). Typical reference schemes in frontal asymmetry studies are the common vertex reference $(\mathrm{Cz})$, computerized linked mastoids (CLM) reference (A1 + A2; see, e.g., Chapter 5), or average scalp reference. Differences between studies in the used reference schemes are often interpreted as mere source of noise (Coan et al., 2006; Hagemann et al., 2001; Reid et al., 1998), yet they may represent a serious threat to the comparability of studies. In particular, the convergent validity of derived frontal asymmetry scores is low in spite of good reliabilities for each method separately (Hagemann et al., 2001). Among the most widely used reference schemes (Cz and 
CLM), it appears that a CLM reference has a somewhat superior signal-to-noise ratio (Coan et al., 2006; Hagemann, 2004). Also, the $\mathrm{Cz}$ reference has a higher contamination with relevant brain activity. This might result in the measurement of a different (and more difficult to interpret) construct, as compared with the CLM reference scheme. Thus, on a theoretical basis, the CLM is preferable among the most common reference schemes (for discussion of other reference techniques, including current source density, see, e.g., Hagemann, 2004; Jaworska et al., 2012; Tenke \& Kayser, 2005). However, for the purpose of this review, the inclusion of studies was not restricted to any particular reference scheme.

\section{Method}

Selection of studies. This review to the best of our knowledge includes all available peer-reviewed studies published in English that reported results of EEG asymmetry in humans, whereby either all participants or a defined subgroup were diagnosed with PTSD, in which case results were reported for each subgroup. The assessment of EEG asymmetry was required to be either a quantitative outcome variable (e.g., with diagnostic status or emotional states as independent variables) or a quantitative factor that was correlated to PTSD symptoms. We used no restriction as to whether resting state or task-related EEG asymmetry was measured. Neither was there a restriction concerning the design in which the measurement of EEG asymmetry was embedded (e.g., single assessment or treatment trials with follow-up measurements).

Search strategy. The Web of Science online search engine (ISI Web of Knowledge; Thompson Reuters, 2014) was used on to find relevant publications. Search terms were applied to Topic search and can be summarized in the formula asymmetr* AND (frontal OR anterior OR eeg OR alpha) AND (PTSD OR *trauma*). No other search restrictions were used. This approach yielded 171 hits in September 2014. Relevance of publications was evaluated by first assessing titles and abstracts provided by the search engine. Relevant studies were assessed in detail to determine whether selection criteria were met. As a second strategy, citing and cited references of selected publications were also assessed for relevance and included when the criteria were met.

Data collection and analysis. Publications were divided into studies that included frontal asymmetry as a trait-like variable (i.e., relying on resting state measures of frontal asymmetry) and those that included state-dependent changes in frontal asymmetry. As another approach to categorize studies, we looked at the type of statistical technique used (e.g., 
correlational analyses between frontal asymmetry and PTSD symptoms vs. group comparisons, e.g., by means of ANOVA). We collected sample characteristics from all studies, including time elapsed since trauma, comorbidity, gender ratio, medication status, age, and methods used to diagnose PTSD or to quantify PTSD symptoms. Also, similar to previous reviews (e.g., Thibodeau et al., 2006) and based on the methodological recommendations summarized above, we recorded the following methodological aspects to evaluate the comparability of the studies: (1) length of EEG recording and number of measurement occasions; (2) the used reference scheme; (3) the electrode sites used to assess frontal asymmetry; and (4) reported procedural details that may be relevant for wanted or unwanted variation in motivational states during the EEG measurements.

\section{Results}

Description of studies. The online search yielded 10 publications that were considered directly relevant to this review. Eight publications fulfilled the inclusion criteria, whereas one study was excluded because PTSD symptoms were not formally assessed (W. J. Curtis \& Cicchetti, 2007) and one study was excluded as it was published in Russian (Kurchakova, Tarabrina, Illarionova, \& Grishkova, 2009). Among the cited references in the included publications, one additional study was relevant but did not fulfil the inclusion criteria (McCaffrey, Lorig, Pendrey, McCutcheon, \& Garrett, 1993; frontal asymmetry derived by period analysis rather than spectral analysis). Among the eight included publications, three stem from the same research group (Rabe, Beauducel, Zöllner, Maercker, \& Karl, 2006; Rabe, Zöllner, Beauducel, Maercker, \& Karl, 2008; Rabe, Zöllner, Maercker, \& Karl, 2006). Three other publications used participant data retrieved from the Brain Resource International Database (BRID; http://brainnet.org.au). It was not possible to calculate the exact number of unique participants, but the most conservative estimate would be that our review pertains to a total of 139 patients diagnosed with PTSD. Additionally, single-occasion frontal asymmetry data were used from healthy or matched control participants $(n=79)$, participants that were exposed to a traumatic situation, but did not develop PTSD $(n=70)$, patients with sub-clinical PTSD symptoms $(n=22)$, and from participants with lifetime, but no current diagnosis of PTSD ( $n=13$ ). One study (Rabe et al., 2008) assessed changes in frontal asymmetry on two occasions in two groups of PTSD patients, one receiving therapy between occasions and the other serving as a waiting list control group. One study (Gordon, Palmer, \& Cooper, 2010) 
compared frontal asymmetry in a PTSD group with normative data from a large control sample. Table 6.2 presents an overview of the included studies.

With respect to analyses of trait frontal asymmetry, six publications (Gordon et al., 2010; Kemp et al., 2010; Rabe, Beauducel, et al., 2006; Rabe et al., 2008; Shankman et al., 2008; Wahbeh \& Oken, 2013) report analyses of group differences in frontal asymmetry at rest, and six publications (Kemp et al., 2010; Metzger et al., 2004; Rabe, Beauducel, et al., 2006; Rabe, Zöllner, et al., 2006; Shankman et al., 2008; Wahbeh \& Oken, 2013) report correlation analyses regarding resting frontal asymmetry and PTSD symptom severity. Gordon et al. (2010) compared frontal asymmetry scores of 48 PTSD patients with normative scores derived from 1908 healthy controls. The average cell sizes in the other group analyses ranged from $n=14.7$ to $n=43.0(M=25.0, S D=11.3)$. Sample sizes in the correlation analyses ranged from $N=14$ to $N=82(M=49.7, S D=25.7)$.

Two publications (Rabe, Beauducel, et al., 2006; Rabe et al., 2008) also focused on state-dependent frontal asymmetry, reporting analyses of group differences in state-dependent frontal asymmetry as well as correlational analyses. Rabe and colleagues addressed statedependent frontal asymmetry using tasks that aimed to activate trauma memories and inductions of trauma-unrelated fear and positive emotions, using images from the International Affective Picture System (IAPS; Lang, Bradley, \& Cuthbert, 2005). 


\begin{tabular}{|c|c|c|c|c|c|c|c|c|}
\hline Authors & $\begin{array}{l}\text { Trait } \\
\text { FA }\end{array}$ & $\begin{array}{l}\text { State } \\
\text { FA }\end{array}$ & Groups & $N$ & $\begin{array}{c}\text { Gender } \\
\text { Ratio M / F }\end{array}$ & Age $(S D)$ & Comorbidity & Medication \\
\hline \multirow{3}{*}{ Metzger et al. (2004) } & Yes & No & PTSD & 16 & $0 / 18$ & NR; about 54 (4) & Current depression & none \\
\hline & & & past PTSD & 13 & $0 / 14$ & & & \\
\hline & & & Trauma non-PTSD & 13 & $0 / 18$ & & & \\
\hline \multirow[t]{4}{*}{ Rabe et al. (2006a) } & Yes & Yes & PTSD & $22^{b}$ & $3 / 19$ & $42,5(11,64)$ & Mood and anxiety & none since $>1$ month \\
\hline & & & Sub-PTSD & $21^{\mathrm{b}}$ & $7 / 14$ & $35,76(9,82)$ & disorders & \\
\hline & & & Trauma non-PTSD & $21^{\mathrm{b}}$ & $10 / 11$ & $43,05(16,63)$ & & \\
\hline & & & Healthy controls & 23 & $7 / 16$ & $37,65(10,72)$ & & \\
\hline \multirow[t]{3}{*}{ Rabe et al. (2006b) } & Yes & No & PTSD & $23^{b}$ & $4 / 19$ & All groups: 41,54 $(13,19)$ & Mood and anxiety & none since $>1$ month \\
\hline & & & Sub-PTSD & $22^{\mathrm{b}}$ & $6 / 16$ & & disorders & \\
\hline & & & Trauma non-PTSD & $37^{\mathrm{b}}$ & $17 / 20$ & & & \\
\hline \multirow[t]{2}{*}{ Rabe et al. (2008) } & Yes & Yes & $\begin{array}{l}\text { PTSD and sub-PTSD } \\
\text { treatment }\end{array}$ & $17^{\mathrm{b}}$ & $2 / 15$ & $38,65(11,47)$ & $\begin{array}{l}\text { Mood and anxiety } \\
\text { disorders }\end{array}$ & none since $>1$ month \\
\hline & & & $\begin{array}{l}\text { PTSD and sub-PTSD } \\
\text { waitlist }\end{array}$ & $18^{\mathrm{b}}$ & $8 / 10$ & $41,89(11,03)$ & & \\
\hline \multirow[t]{2}{*}{ Shankman et al. (2008) } & Yes & No & PTSD & $32^{\mathrm{c}}$ & $16 / 16$ & $40,7(11,3)$ & NR & NR; present in "many" \\
\hline & & & Healthy controls $^{\mathrm{a}}$ & $42^{\mathrm{c}}$ & $27 / 15$ & $36,0(14,0)$ & & cases (p. 196) \\
\hline \multirow[t]{3}{*}{ Kemp et al. (2010) } & Yes & No & PTSD & $14^{\mathrm{b}}$ & $5 / 9$ & $41,4(12,3)$ & NR & antidepressants \\
\hline & & & Depression & 15 & $6 / 9$ & $39,9(14,0)$ & & \\
\hline & & & Healthy controls & $15^{\mathrm{d}}$ & $6 / 9$ & $42,4(16,7)$ & & \\
\hline \multirow[t]{2}{*}{ Gordon et al. (2010) } & Yes & No & PTSD & $48^{\mathrm{c}}$ & $25 / 23$ & NR & NR & NR \\
\hline & & & Healthy controls & $1908^{c}$ & $971 / 937$ & NR; range: 6-87 & & \\
\hline \multirow[t]{2}{*}{ Wahbeh and Oken (2013) } & Yes & No & PTSD & $59^{\mathrm{e}}$ & $59 / 0$ & $54,4(11,5)$ & NR & Stable > 1 month \\
\hline & & & Trauma non-PTSD & $27^{\mathrm{e}}$ & $27 / 0$ & $53,1(11,3)$ & & \\
\hline
\end{tabular}

Table 6.2. Summary of focus and sample characteristics of the included studies. FA = frontal asymmetry; $\mathrm{M} / \mathrm{F}=$ male / female; NR = not reported;

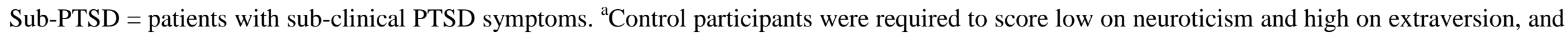

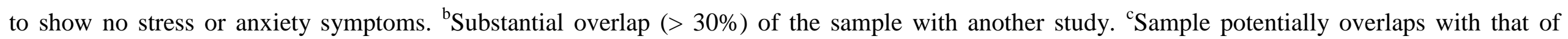
another study, but the proportion could not be determined. ${ }^{\mathrm{d}}$ Partial overlap $(<30 \%)$ of the sample with another study. ${ }^{\mathrm{e}}$ Seven participants from the combined sample were excluded from the analyses. 
Methodological issues. The characteristics of the included samples are summarized in Table 6.2. In all studies, the Clinician Administered PTSD Scale (CAPS; Blake et al., 1995) was used as the basis for group allocation (e.g., PTSD, resilient) and / or to quantify PTSD symptom severity. Type of trauma was war experiences in two studies (Metzger et al., 2004; Wahbeh \& Oken, 2013), motor vehicle accidents in the three Rabe et al. studies, and various or non-specified trauma types in the remaining studies (Gordon et al., 2010; Kemp et al., 2010; Shankman et al., 2008). Notably, while three studies do not report on comorbidity (Gordon et al., 2010; Kemp et al., 2010; Shankman et al., 2008), comorbid mood and anxiety disorders were present in the other studies. Current psychoactive medication was present in three studies (Kemp et al., 2010; Shankman et al., 2008; Wahbeh \& Oken, 2013), not reported in one (Gordon et al., 2010), and absent in the other studies.

Table 6.3 summarizes methodological details regarding the EEG recordings in each study. As can be seen, most studies used the CLM reference scheme, whereas two used an average reference scheme (Shankman et al., 2008; Wahbeh \& Oken, 2013). Most studies focused on lateral frontal electrodes for the measurement of frontal asymmetry, including the paired electrode sites $\mathrm{F} 3 / \mathrm{F} 4$, FC1/FC2, or FC3/FC4, whereas two studies from the Rabe group (Rabe, Beauducel, et al., 2006; Rabe et al., 2008) also included more lateral and more posterior electrodes for the calculation of frontal asymmetry, by averaging the power density values of F3, F7, and T7 as well as F4, F8, and T8. Recording length in trait frontal asymmetry studies ranged from 120 - $480 \mathrm{~s}$ across studies. Measurements were restricted to one occasion in all studies, although Rabe et al. (2008) used measurements from two occasions to derive frontal asymmetry change scores. All assessments of state frontal asymmetry were based on $60 \mathrm{~s}$ measurements during mood inductions.

Reported effects. The reported results concerning trait frontal asymmetry are summarized in Table 6.4. As can be seen, all studies failed to find significant group differences in trait frontal asymmetry. Likewise, all but one study found small and nonsignificant correlations between trait frontal asymmetry and PTSD symptoms (i.e., CAPS scores) in PTSD patients or across trauma groups. By contrast, the Kemp et al. (2010) study reports a relatively large negative correlation $(r=-.62)$ between frontal asymmetry and CAPS scores, indicating that more left-sided frontal activity was associated with fewer PTSD symptoms. Because of overlapping participant samples in the studies, we were not able to calculate a valid average correlation between frontal asymmetry and CAPS scores. 


\begin{tabular}{|c|c|c|c|c|c|c|}
\hline Authors & Reference & Frontal Sites & State/Trait & Occasions & Recording Length (s) & Procedural Details \\
\hline \multirow[t]{2}{*}{ Rabe et al. (2006a) } & CLM & $\begin{array}{l}\text { Avg F3, F7, T7 / } \\
\text { Avg F4, F8, T8 }\end{array}$ & State & 1 & 60 & $\begin{array}{l}60 \mathrm{~s} \text { periods for each mood } \\
\text { induction, } 120 \mathrm{~s} \text { pause between } \\
\text { inductions }\end{array}$ \\
\hline & & & Trait & 1 & 480 & $4 \min \mathrm{EO}, 4 \min \mathrm{EC}$ \\
\hline Rabe et al. (2006b) & CLM & $\mathrm{FC} 1 / \mathrm{FC} 2$ & Trait & 1 & 480 & $4 \min \mathrm{EO}, 4 \min \mathrm{EC}$ \\
\hline \multirow[t]{2}{*}{ Rabe et al. (2008) } & CLM & $\begin{array}{c}\text { Avg F3, F7, T7 / } \\
\text { Avg F4, F8, T8 }\end{array}$ & State & 2 (change) & 60 & $\begin{array}{l}60 \mathrm{~s} \text { periods for each mood } \\
\text { induction, } 120 \mathrm{~s} \text { pause between } \\
\text { inductions }\end{array}$ \\
\hline & & & Trait & 2 (change) & 480 & $4 \min \mathrm{EO}, 4 \min \mathrm{EC}$ \\
\hline $\begin{array}{l}\text { Shankman et al. } \\
(2008)\end{array}$ & $\begin{array}{l}\text { Scalp average (24 } \\
\text { electrodes) }\end{array}$ & F3 / F4 & Trait & 1 & 240 & $2 \min \mathrm{EO}, 2 \min \mathrm{EC}$ \\
\hline Kemp et al. (2010) & CLM & F3 / F4 & Trait & 1 & 120 & $2 \min \mathrm{EC}$ \\
\hline $\begin{array}{l}\text { Gordon et al. } \\
\text { (2010) }\end{array}$ & CLM & $\mathrm{FC} 3 / \mathrm{FC} 4$ & Trait & 1 & 240 & $2 \min \mathrm{EO}, 2 \min \mathrm{EC}$ \\
\hline
\end{tabular}

Table 6.3. Methodological details of the included studies. EO = eyes open; EC = eyes closed. 
Table 6.5 summarizes the results concerning state-dependent frontal asymmetry. As can be seen, PTSD patients displayed significantly stronger right-sided activation in response to trauma-related stimuli in the Rabe, Beauducel, et al. (2006) study. Moreover, the latter study found a significant positive correlation between relatively more right-sided activation in this condition and CAPS scores. Additionally, Rabe et al. (2008) found that relatively right-sided activation in response to trauma-related stimuli decreased significantly in patients who received cognitive-behavioural treatment (CBT), but this decrease was only marginally stronger than that in the waitlist control group. Moreover, changes in frontal asymmetry across both groups were unrelated to improvement in PTSD symptoms. However, the decrease in right-sided activation (irrespective of left-sided activation) was significantly associated with a reduction in PTSD symptoms. With respect to the induction of positive emotion and trauma-unrelated fear, the two studies found no group differences in activation asymmetry, no correlation of activation asymmetry with PTSD symptoms, and no changes in activation asymmetry attributable to CBT. 


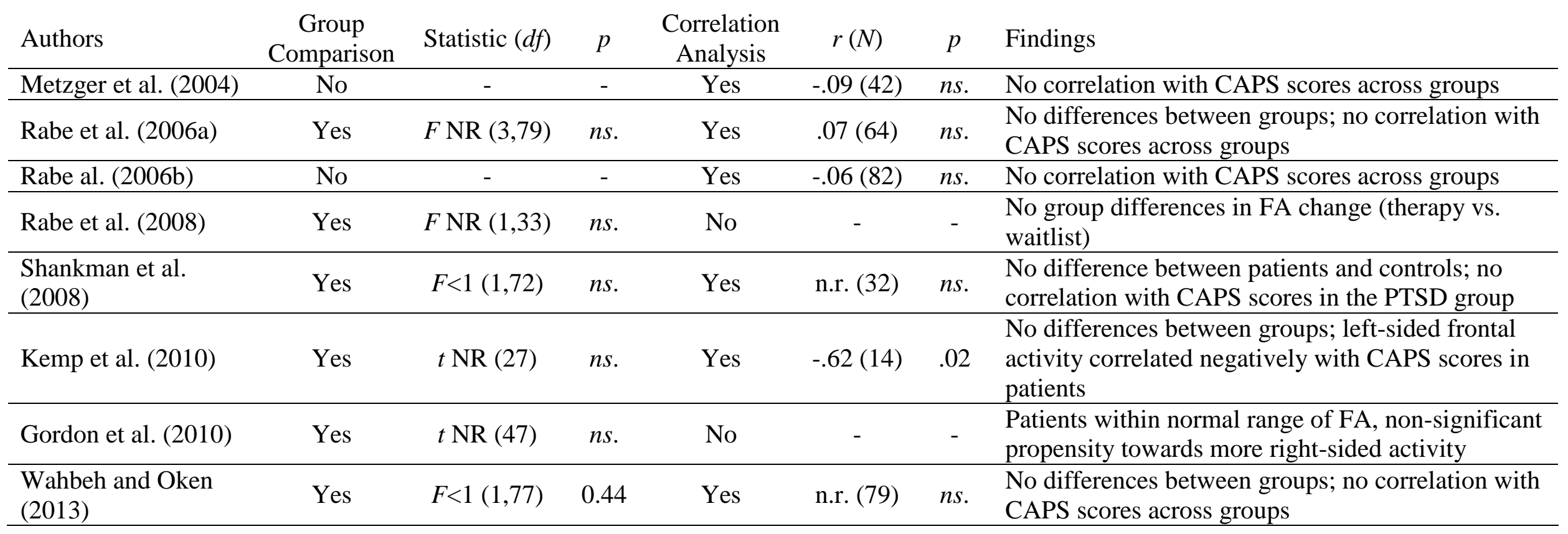

Table 6.4. Trait frontal asymmetry: Correlations and group analyses Note. Dashes indicate that the statistic is not available because the analysis was not performed. NR = note reported; CAPS = Clinician Administered PTSD Scale; FA = frontal asymmetry. 


\begin{tabular}{|c|c|c|c|c|c|c|c|c|}
\hline Authors & Induction $^{\mathrm{a}}$ & ANOVA & $F(d f)$ & $p$ & $\begin{array}{l}\text { Correlation } \\
\text { Analysis }\end{array}$ & $r(N)$ & $p$ & Findings \\
\hline \multirow{2}{*}{$\begin{array}{l}\text { Rabe et al. } \\
(2006 a)\end{array}$} & Fear & $\begin{array}{l}\text { FA between } \\
\text { groups }\end{array}$ & NR $(3,79)$ & $n s$. & FA with CAPS & $-.05(64)$ & $n s$ & $\begin{array}{l}\text { No group differences in activation FA; no } \\
\text { association with CAPS scores }\end{array}$ \\
\hline & Positive & $\begin{array}{l}\text { FA between } \\
\text { groups }\end{array}$ & NR $(3,79)$ & $n s$. & FA with CAPS & $-.09(64)$ & $n s$. & $\begin{array}{l}\text { No group differences in activation FA; no } \\
\text { association with CAPS scores }\end{array}$ \\
\hline \multirow[t]{3}{*}{$\begin{array}{l}\text { Rabe et al. } \\
(2008)\end{array}$} & Trauma & $\begin{array}{l}\text { FA change } \\
\text { between groups }\end{array}$ & $3.49(1,33)$ & .07 & $\begin{array}{l}\text { FA change with } \\
\text { CAPS change }\end{array}$ & $.08(35)$ & .44 & $\begin{array}{l}\text { Trend-significant effect of treatment vs. } \\
\text { waitlist on FA change, but significant FA } \\
\text { change within treatment group; change in } \\
\text { FA unrelated to CAPS change, but } \\
\text { decrease in right-sided activation } \\
\text { associated with reduction of CAPS scores. }\end{array}$ \\
\hline & Fear & $\begin{array}{l}\text { FA change } \\
\text { between groups }\end{array}$ & NR $(1,33)$ & $n s$. & No & - & - & $\begin{array}{l}\text { No effect of treatment vs. waitlist on FA } \\
\text { change }\end{array}$ \\
\hline & Positive & $\begin{array}{l}\text { FA change } \\
\text { between groups }\end{array}$ & NR $(1,33)$ & $n s$. & No & - & - & $\begin{array}{l}\text { No effect of treatment vs. waitlist on FA } \\
\text { change }\end{array}$ \\
\hline
\end{tabular}

Table 6.5. State-dependent frontal asymmetry: Correlations and group analyses. Dashes indicate that the statistic is not available because the analysis was not performed. FA = frontal asymmetry; CAPS = Clinician Administered PTSD Scale; Sub-PTSD = patients with sub-clinical PTSD symptoms; R-change = change in right frontal activation; L-change = change in left frontal activation; NR = not reported. ${ }^{\mathrm{a}}$ Emotional states were induced by presenting standardized pictures for $60 \mathrm{~s}$. The state-dependent frontal asymmetry scores used in the analyses reflect increased right-sided activation (decreased right-sided alpha power) relative to a control condition in which a neutral picture was shown. 


\section{Discussion}

In this article, we aimed to explore whether and how frontal asymmetry may be related to individual differences in adjustment to traumatic experiences and, in particular, whether it may serve as a biomarker of PTSD. We therefore first reviewed the frontal asymmetry literature and outlined its possible roles in cognition, emotion, and psychopathology in general. This literature can be broadly divided into studies that measured frontal asymmetry at rest, aiming to assess stable individual differences, and studies measuring state-dependent frontal asymmetry in response to a specific emotional stimulation. Both trait and statedependent frontal asymmetry appear to reflect motivational direction, with more left-sided frontal activity reflecting approach and more right-sided activity reflecting withdrawal motivation. As well, both indices seem relevant and complementary for our understanding of individual differences in emotional processing and psychopathology.

The available literature suggests that trait and state frontal asymmetry are related to current and lifetime depression, as well as to the risk to develop depression. Additionally, there is good evidence to suggest that frontal asymmetry may be similarly indicative of anxiety disorders, the risk to develop an anxiety disorder, and cognitive biases that could underlie anxiety disorders. PTSD shares fear-related symptoms with other anxiety disorders, dysphoria-related symptoms with depression, and general distress symptoms with both types of disorder. On this basis, it is not farfetched to assume that frontal asymmetry plays a role in PTSD as well.

We also explored the neural origins of frontal asymmetry and how they might be related to the neurocircuitry of PTSD. From this perspective, frontal asymmetry has been proposed to reflect differential hemispheric activity in specific brain regions, including the left and right dlPFC, vmPFC, and the amygdala, but frontal asymmetry could also reflect a more global and unspecific bias towards lateralized brain functions. The brain regions thought to be predominantly responsible for frontal asymmetry are usually implicated in emotion regulation, memory, and automatic affective responding, and have also been found to function abnormally in PTSD patients. Although neuroimaging studies rarely performed explicit tests of laterality, several plausible links emerge between relatively right-sided frontal asymmetry and the neurocircuitry of this disorder (see Figure 6.1 and Table 6.1).

Finally, we reviewed empirical studies that directly assessed whether PTSD is marked by abnormal frontal EEG asymmetry. Of eight publications that fulfilled our criteria, all 
included measures of trait frontal asymmetry, while two additionally focused on statedependent frontal asymmetry, in relation to PTSD symptoms. Surprisingly, only one study found a relationship between trait frontal asymmetry and PTSD symptoms, with increased right-sided frontal activity predicting more severe symptoms. The two publications focusing on state-dependent frontal asymmetry (Rabe, Beauducel, et al., 2006; Rabe et al., 2008) found that higher right-sided frontal activation in response to trauma-related stimuli, but not unrelated negative stimuli, correlates with PTSD symptoms. Together, these data suggest that state frontal asymmetry during exposure to trauma-relevant stimuli, but not trait frontal asymmetry, may distinguish PTSD patients from resilient and from trauma-free individuals.

\section{Methodological issues}

Importantly, the studies included in our review have methodological weaknesses that merit comment. Trait frontal asymmetry correlated with PTSD symptoms only in one study, and within this study only in the PTSD patient subgroup ( $n=14$; Kemp et al., 2010). Notably, besides a smaller sample size than the other studies (see Table 6.2 and 6.3), this study also had the shortest recording period. This is a potential weakness, since shorter recording periods decrease the trait specificity of the resting state measure (Hagemann, 2004). Interestingly, however, a meta-analysis by Thibodeau and colleagues (2006) found that length of EEG recording was negatively associated with the effect size for the relationship between frontal asymmetry and depression. This suggests that trait frontal asymmetry correlates with psychopathology especially when the measurement is more prone to contamination by state influences in the laboratory, such as negative mood shifts due to the EEG preparation, the sex of the experimenter, or the time of day and the time of year (Hagemann, 2004; Harmon-Jones et al., 2010; Velo, Stewart, Hasler, Towers, \& Allen, 2012). As none of the reviewed studies measured such factors, their potentially moderating role still remains unclear.

Another limitation of our review is the small number of available studies that precludes definitive conclusions about whether methodological variations affected the trait frontal asymmetry results. In particular, length of EEG recording, recording sites used to derive frontal asymmetry varied considerably between studies, as did sample characteristics such as the gender ratio. Related to this concern, the current review included studies from only four entirely independent research programs. That is, three studies were from the same research group, whereas three other publications used parts of a common participant database. As a consequence, some of the included publications are based on overlapping participant samples 
(see Table 6.2), warranting additional caution in generalizing the results. Similarly, the conclusions regarding state-dependent frontal asymmetry are limited by the fact that all findings come from a single laboratory and are based on partly overlapping samples.

\section{Resting frontal asymmetry as a marker of PTSD}

We found no robust correlation between trait frontal asymmetry and PTSD symptoms, and this seems to indicate that trait frontal asymmetry cannot serve as a reliable biomarker of PTSD. Notably, this conclusion could be premature in light of methodological limitations (cf. supra). Instead, it is possible that the strong heterogeneity of symptoms could have overshadowed potential effects, as well as the typically high level of comorbidity that we also found in the included studies (see Table 6.2). In line with this, Thibodeau et al. (2006) found that right-sided frontal activity, indeed, characterizes patients with depression or with an anxiety disorder, but lower correlations were found in patients with comorbid anxiety and depression. Thus, the complex clinical picture of PTSD, which features symptoms of both anxiety and depression, may have reduced the correlation patterns in research on frontal asymmetry.

According to this line of reasoning, different PTSD symptom clusters may be differentially associated with trait frontal asymmetry. On the basis of the motivational direction model of frontal asymmetry (Davidson, 1998a), one could expect fear-related symptoms to be associated with exaggerated withdrawal motivation and hence, with increased right-sided activity. Similarly, dysphoria-related symptoms could be related to deficient approach motivation and decreased left-sided activity. Meanwhile, avoidance-related symptoms could have an ambivalent relationship with frontal asymmetry. That is, these symptoms may be driven by motivations to withdraw (i.e., fear) on the one hand, and on the other hand, by approach-related goals subserving an active avoidance strategy (i.e., engaging in active behaviours to undo fear). Finally, it is still unclear how frontal asymmetry might be related to dissociative amnesia and flashbacks, which are symptoms that may uniquely distinguish PTSD from other mental disorders (Brewin, 2011; Bryant et al., 2011).

In addition to the broad symptom clusters, an important observation might be that traumatized individuals often display exaggerated anger or related emotions, such as bitterness and erroneous self- or other blame. These reactions often lead to reckless, aggressive, and maladaptive behaviour and have a major impact on post-traumatic adjustment (Friedman et al., 2011; Linden, Baumann, Rotter, \& Schippan, 2008; McHugh, Forbes, Bates, 
Hopwood, \& Creamer, 2012). Importantly, both state and trait anger are marked by approach motivation and have been associated with left-sided frontal activity (Harmon-Jones et al., 2010; but see Jaworska et al., 2012). In other words, extreme approach and withdrawal tendencies can coexist in PTSD, and this may be expressed in opposing patterns of frontal asymmetry. Since approach and withdrawal tendencies are thought to transiently suppress each other (Schutter \& Harmon-Jones, 2013), anger-related symptoms might dampen or reverse frontal asymmetry in resting state measurements. Thus, on the one hand, future studies should explicitly take anger-related symptoms into account. On the other hand, it appears generally advisable to control for transient motivations in measurements of frontal asymmetry, implying that state-dependent measures might be preferable over resting state measures. Related to this, future research might try to address the flow of the causal directions in frontal asymmetry. That is, would activity asymmetries be driven by increased use of cognitive functions in one hemisphere or by impaired or inefficient processing in the other hemisphere? By making such issues explicit, theories concerning frontal asymmetry and the hypotheses formulated in this review (see Table 1) would become more testable.

Finally, it is worth mentioning that a number of other factors might moderate the association between frontal asymmetry and PTSD. For instance, trauma type and severity affect peri-traumatic stress and risk perception (A. Grimm, Hulse, Preiss, \& Schmidt, 2012) and can change the course of the disorder (Brewin et al., 2000). According to McNally and Robinaugh (2011), trauma type and severity might also make individual risk and resilience factors more or less important in determining mental health outcomes. This might also apply to the association between frontal asymmetry and PTSD. Suggestive support for this view comes from our study in Chapter 5 using trauma films, in which we found that frontal asymmetry is associated with startle responses to film cues, but size and direction of this effect was moderated by the type of trauma film. Taken together, more controlled studies of frontal asymmetry in relation to more specific patient groups, symptom clusters and underlying factors (e.g., depression, anxiety, and anger), as well as transient states are both promising and pressingly needed (for similar discussions, see Bonanno, Westphal, \& Mancini, 2011; Van Praag et al., 1990).

\section{State-dependent frontal asymmetry and PTSD}

The pattern of findings reported by Rabe and colleagues (Rabe, Beauducel, et al., 2006; Rabe, Zöllner, et al., 2006) suggests that PTSD patients might be characterized by an 
abnormal right-sided frontal activation in response to trauma-related stimuli. Meanwhile, PTSD patients did not display abnormal asymmetric activation to unrelated negative stimuli or activity at rest. This pattern is reminiscent of the finding that many PTSD patients display abnormally strong startle responses, specifically to trauma-related stimuli (Orr \& Roth, 2000), which is indicative of enhanced amygdala activation (Davis, Walker, \& Lee, 1997). Thus, one may hypothesize that right-sided frontal activation in response to trauma-related stimuli is a down-stream effect of exaggerated negative information processing as well as autonomic and neuroendocrine stress reactions mediated by the right vmPFC and amygdala (see, e.g., Cerqueira et al., 2008; Greenberg et al., 2005). Of course, some caution is warranted, as the idea that PTSD symptom correspond with exaggerated right-hemisphere responding still awaits more direct empirical scrutiny (for a review, see Francati et al., 2007). Taken together, this appealing interpretation warrants further investigation.

Should future studies corroborate the findings of Rabe and colleagues (Rabe, Beauducel, et al., 2006; Rabe, Zöllner, et al., 2006), then practical implications can be envisioned: statedependent frontal asymmetry in response to trauma-related cues could serve as a biological marker of PTSD symptoms. Importantly, developing an objective measure tapping into PTSD symptoms would be of great value, as it could facilitate and improve diagnosis and guide future research on PTSD (Rosen \& Lilienfeld, 2008). Note, for example, that self-report measures of PTSD symptoms are sensitive to the distortive effects of symptom exaggerations (Frueh, Hamner, Cahill, Gold, \& Hamlin, 2000). It is intuitively plausible to assume that a frontal asymmetry index is immune to such response bias effects.

The next step in the development of an objective marker would be to investigate how the emotional challenge can be optimized for diagnostic purposes. Rabe and colleagues found associations between PTSD symptoms and frontal asymmetry when participants were exposed to a relevant stimulus (i.e., an image of a car accident) that activated memories of trauma (motor vehicle accidents). By contrast, frontal asymmetry during the viewing of an unrelated negative stimulus (i.e., an image of a barking dog) was unrelated to symptoms. Consequently, it remains to be seen whether asymmetric activation in response to other (e.g., stronger) negative stimuli that are not (yet) idiosyncratically linked to a traumatic event could predict PTSD. As well, future research will need to explore the sensitivity and specificity of the elicited asymmetric responses to PTSD. That is, more right-sided frontal activation in response to emotional challenges is also evident in patients with depression (Stewart et al., 2011) and panic disorder (Wiedemann et al., 1999), suggesting that this could be a transdiagnostic marker of emotional symptoms. Therefore, a more fine-grained analysis of the 
specific symptom clusters that are associated with state-dependent frontal asymmetries would be informative.

\section{Other promising avenues for frontal asymmetry research}

Several recent advances in the field of frontal asymmetry research that could notably benefit the study of this potential marker of psychopathology deserve to be mentioned. One promising route for future research is to refine the measurement of frontal asymmetry. For instance, Allen and Cohen (2010) measured short bursts of alpha asymmetry instead of averaging alpha power across several minutes. This way, the authors aimed to increase the temporal specificity of the asymmetry metric, which could eventually help to understand the neuropsychological underpinnings of frontal asymmetry and to better link it to neuroanatomical data. Others have focused on individual alpha frequencies (IAF; typically ranging from 9.5-11.5 Hz in young healthy adults; Klimesch, 1999) for deriving alpha asymmetry scores, rather than using the broader alpha frequency band from 8-13 Hz (see, e.g., Quaedflieg et al., 2015; Segrave et al., 2011). Similarly, it might be beneficial to investigate frontal asymmetry separately in two alpha sub-bands, since desynchronization in the lower alpha range (below IAF) has been suggested to reflect attentional processes, whereas desynchronization in the upper alpha range (above IAF) might reflect cognitive activity (Klimesch, 1999). Finally, with the rapidly advancing understanding of the functional significance of neuronal oscillations in brain networks (Buzsaki \& Draguhn, 2004; Klimesch, 1999; Klimesch et al., 2007), it may become increasingly interesting to consider frontal asymmetry in the alpha band in relation to (lateralized) synchronization in other bands (e.g., asymmetric gamma activity might be related to memory processes; Babiloni et al., 2006; activity in the theta band might reflect limbic activity; Klimesch, 1999; for a discussion, see also Miller, Crocker, Spielberg, Infantolino, \& Heller, 2013).

More generally, and as suggested by Davidson (2004), this type of research will likely benefit from combining EEG with other cognitive neuroscience techniques by elucidating the more detailed neuronal basis of asymmetric electrical signals, and from interventions that help in understanding the causal role of frontal asymmetry in emotion. As we stated in our review, non-EEG neuroimaging studies often omit explicit tests of laterality. Adding these would clearly help to advance our understanding of brain asymmetry. For most brain functions, we would not expect research to identify a distinctive "division of labor" between the two hemispheres, because homologous structures in the two hemispheres typically show 
functional overlap. Still, laterality effects can be highly meaningful and are worth exploring further. One type of study that has already provided valuable insights about lateralized brain functions and emotion processing is using rTMS as an intervention to (asymmetrically) change brain activity (Baeken, Van Schuerbeek, et al., 2011; Baeken, Vanderhasselt, et al., 2011; Leyman et al., 2009; Vanderhasselt, Baeken, Hendricks, \& De Raedt, 2011).

Another promising intervention technique is EEG neurofeedback, in which parameters of the brain's electrophysiology are used as the basis for real-time feedback to the participant. By this feedback, individuals undergoing neurofeedback learn to self-regulate specific patterns of their brain physiology. Neurofeedback has already been employed to change frontal asymmetry, but the impact of this on different emotional responses remains to be evaluated empirically (cf., Allen, Harmon-Jones, \& Cavender, 2001; Peeters, Ronner, Bodar, van Os, \& Lousberg, 2014). Yet, a number of case studies (Baehr, Rosenfeld, \& Baehr, 1997, 2001) as well as a randomized treatment trial in depression (Choi et al., 2011) suggest that this approach may be beneficial in the therapy of emotional disorders.

\section{Conclusions}

A large and growing body of literature strongly suggests that both state-dependent and trait frontal asymmetries can be informative markers related to depression and anxiety disorders. This article evaluated the relevance of this biological marker for PTSD. We show that the possible neuroanatomical origins of frontal asymmetry that are proposed in the literature can be plausibly linked to abnormal psychological and brain functions thought to be responsible for PTSD. We thus formulated a set of new hypotheses concerning the neural processes that may connect state-dependent and trait frontal asymmetry to psychological adjustment after trauma. Next, from our review, we also conclude that surprisingly few studies have directly addressed the relationship between frontal asymmetry and PTSD. The data suggest that trait frontal asymmetry has little if any predictive value with respect to PTSD symptoms, whereas frontal activation after trauma-relevant stimulation might be a marker of PTSD. Of course, replications and extensions of these studies are needed before the practical and theoretical relevance of this biological marker can be fully evaluated. In addition, we suggest focusing on specific PTSD symptoms, particularly anxiety, dysphoria, and anger-related symptoms, in relation to frontal asymmetry. Finally, future research will determine whether frontal asymmetry can serve as a diagnosis-specific or transdiagnostic marker of pathological adjustment. 


\section{References}

Ahern, G. L., Sollers, J. J., Lane, R. D., Labiner, D. M., Herring, A. M., Weinand, M. E., . . . Thayer, J. F. (2001). Heart rate and heart rate variability changes in the intracarotid sodium amobarbital test. Epilepsia, 42, 912-921. doi: 10.1046/j.15281157.2001.042007912.x

Allen, J. J. B., \& Cohen, M. X. (2010). Deconstructing the "resting" state: Exploring the temporal dynamics of frontal alpha asymmetry as an endophenotype for depression. Frontiers in Human Neuroscience, 4, 14. doi: 10.3389/fnhum.2010.00232

Allen, J. J. B., Harmon-Jones, E., \& Cavender, J. H. (2001). Manipulation of frontal EEG asymmetry through biofeedback alters self-reported emotional responses and facial EMG. Psychophysiology, 38, 685-693. doi: 10.1111/1469-8986.3840685

Allen, J. J. B., \& Kline, J. P. (2004). Frontal EEG asymmetry, emotion, and psychopathology: The first, and the next 25 years. Biological Psychology, 67, 1-5. doi: 10.1016/j.biopsycho.2004.03.001

American Psychiatric Association. (2013). Diagnostic and statistical manual of mental disorders (5th ed.). Arlington, VA: American Psychiatric Publishing.

Asbjornsen, A. E. (2011). Dichotic listening performance suggests right hemisphere involvement in PTSD. Laterality, 16, 401-422. doi: 10.1080/13576501003702655

Avram, J., Baltes, F. R., Miclea, M., \& Miu, A. C. (2010). Frontal EEG activation asymmetry reflects cognitive biases in anxiety: Evidence from an emotional face stroop task. Applied Psychophysiology and Biofeedback, 35, 285-292. doi: $10.1007 / \mathrm{s} 10484.010 .9138 .6$

Babiloni, C., Babiloni, F., Carducci, F., Cappa, S., Cincotti, F., Del Percio, C., . . Rossini, P. M. (2004). Human cortical EEG rythms during long-term episodic memory task. A high-resolution EEG study of the HERA model. NeuroImage, 21, 1576-1584. doi: 10.1016/j.neuroimage.2003.11.023

Babiloni, C., Vecchio, F., Cappa, S., Pasqualetti, P., Rossi, S., Miniussi, C., \& Rossini, P. M. (2006). Functional frontoparietal connectivity during encoding and retrieval processes follows HERA model - A high-resolution study. Brain Research Bulletin, 68, 203-212. doi: 10.1016/j.brainresbull.2005.04.019

Baehr, E., Rosenfeld, J. P., \& Baehr, R. (1997). The clinical use of alpha asymmetry biofeedback protocol in treatment of depressive disorders: Two case studies. Journal of Neurotherapy, 2, 12-27. 
Baehr, E., Rosenfeld, J. P., \& Baehr, R. (2001). Clinical use of an alpha asymmetry neurofeedback protocol in the treatment of mood disorders: Follow-up study one to five years post therapy. Journal of Neurotherapy, 4, 11-18.

Baeken, C., Van Schuerbeek, P., De Raedt, R., De Mey, J., Vanderhasselt, M. A., Bossuyt, A., \& Luypaert, R. (2011). The effect of one left-sided dorsolateral prefrontal shamcontrolled HF-rTMS session on approach and withdrawal related emotional neuronal processes. Clinical Neurophysiology, 122, 2217-2226. doi: 10.1016/j.clinph.2011.04.009

Baeken, C., Vanderhasselt, M. A., \& De Raedt, R. (2011). Baseline 'state anxiety' influences HPA-axis sensitivity to one sham-controlled HF-rTMS session applied to the right dorsolateral prefrontal cortex. Psychoneuroendocrinology, 36, 60-67. doi: 10.1016/j.psyneuen.2010.06.006

Barnhofer, T., Duggan, D., Crane, C., Hepburn, S., Fennell, M. J. V., \& Williams, J. M. G. (2007). Effects of meditation on frontal alpha-asymmetry in previously suicidal individuals. Neuroreport, 18, 709-712. doi: 10.1097/WNR.0b013e3280d943cd

Beauregard, M., Levesque, J., \& Bourgouin, P. (2001). Neural correlates of conscious selfregulation of emotion. Journal of Neuroscience, 21, 6. doi: Rc165

Berkman, E. T., \& Lieberman, M. D. (2010). Approaching the bad and avoiding the good: Lateral prefrontal cortical asymmetry distinguishes between action and valence. Journal of Cognitive Neuroscience, 22, 1970-1979.

Blake, D. D., Weathers, F. W., Nagy, L. M., Kaloupek, D. G., Gusman, F. D., Charney, D. S., \& Keane, T. M. (1995). The development of a clinician-administered PTSD scale. Journal of Traumatic Stress, 8, 75-90. doi: 10.1007/bf02105408

Bluhm, R. L., Williamson, P. C., Osuch, E. A., Frewen, P. A., Stevens, T. K., Boksman, K., . . . Lanius, R. A. (2009). Alterations in default network connectivity in posttraumatic stress disorder related to early-life trauma. Journal of Psychiatry \& Neuroscience, 34, 187.

Bonanno, G. A. (2004). Loss, trauma, and human resilience - Have we underestimated the human capacity to thrive after extremely aversive events? American Psychologist, 59, 20-28. doi: 10.1037/0003-066x.59.1.20

Bonanno, G. A. (2012). Uses and abuses of the resilience construct: Loss, trauma, and healthrelated adversities. Social Science \& Medicine, 74, 753-756. doi: 10.1016/j.socscimed.2011.11.022 
Bonanno, G. A., \& Mancini, A. D. (2008). The human capacity to thrive in the face of potential trauma. Pediatrics, 121, 369-375. doi: 10.1542/peds.2007-1648

Bonanno, G. A., Westphal, M., \& Mancini, A. D. (2011). Resilience to loss and potential trauma. In S. Nolen-Hoeksema, T. D. Cannon \& T. Widiger (Eds.), Annual review of clinical psychology (Vol. 7, pp. 511-535). Palo Alto: Annual Reviews.

Brewin, C. R. (2011). The nature and significance of memory disturbance in posttraumatic stress disorder. Annual Review of Clinical Psychology, 7, 203-227. doi:10.1146/annurev-clinpsy-032210-104544

Brewin, C. R., Andrews, B., \& Valentine, J. D. (2000). Meta-analysis of risk factors for posttraumatic stress disorder in trauma-exposed adults. Journal of Consulting and Clinical Psychology, 68, 748-766.

Brewin, C. R., Gregory, J. D., Lipton, M., \& Burgess, N. (2010). Intrusive images in psychological disorders: Characteristics, neural mechanisms, and treatment implications. Psychological Review, 117, 210-232. doi: 10.1037/a0018113

Browning, M., Holmes, E. A., Murphy, S. E., Goodwin, G. M., \& Harmer, C. J. (2010). Lateral prefrontal cortex mediates the cognitive modification of attentional bias. Biological Psychiatry, 67, 919-925. doi: 10.1016/j.biopsych.2009.10.031

Bryant, R. A., O’Donnell, M. L., Creamer, M., McFarlane, A. C., \& Silove, D. (2011). Posttraumatic intrusive symptoms across psychiatric disorders. Journal of Psychiatric Research, 45, 842-847. doi: 10.1016/j.jpsychires.2010.11.012

Buzsaki, G., \& Draguhn, A. (2004). Neuronal oscillations in cortical networks. Science, 304, 1926-1929. doi: 10.1126/science. 1099745

Cabeza, R., \& St Jacques, P. (2007). Functional neuroimaging of autobiographical memory. Trends in Cognitive Sciences, 11, 219-227. doi: 10.1016/j.tics.2007.02.005

Cerqueira, J. J., Almeida, O. F. X., \& Sousa, N. (2008). The stressed prefrontal cortex. Left? Right! Brain Behavior and Immunity, 22, 630-638. doi: 10.1016/j.bbi.2008.01.005

Cerqueira, J. J., Mailliet, F., Almeida, O. F. X., Jay, T. M., \& Sousa, N. (2007). The prefrontal cortex as a key target of the maladaptive response to stress. Journal of Neuroscience, 27, 2781-2787. doi: 10.1523/jneurosci.4372-06.2007

Chen, J. J., Zhou, C. J., Wu, B., Wang, Y., Li, Q., Wei, Y. D., . . Xie, P. (2013). Left versus right repetitive transcranial magnetic stimulation in treating major depression: A metaanalysis of randomised controlled trials. Psychiatry Research, 210, 1260-1264. doi: 10.1016/j.psychres.2013.09.007 
Choi, S. W., Chi, S. E., Chung, S. Y., Kim, J. W., Ahn, C. Y., \& Kim, H. T. (2011). Is alpha wave neurofeedback effective with randomized clinical trials in depression? A pilot study. Neuropsychobiology, 63, 43-51.

Choudhary, C. J., \& O'Carroll, R. E. (2007). Left hand preference is related to posttraumatic stress disorder. Journal of Traumatic Stress, 20, 365-369. doi: 10.1002/jts.20222

Clark, C. R., McFarlane, A. C., Morris, P., Weber, D. L., Sonkkilla, C., Shaw, M., . . Egan, G. F. (2003). Cerebral function in posttraumatic stress disorder during verbal working memory updating: A positron emission tomography study. Biological Psychiatry, 53, 474-481. doi: 10.1016/S0006-3223(02)01505-6

Coan, J. A., \& Allen, J. J. B. (2003). The state and trait nature of frontal EEG asymmetry in emotion. In K. Hugdahl \& R. J. Davidson (Eds.), The asymmetrical brain (pp. 565615). Cambridge: MIT Press.

Coan, J. A., \& Allen, J. J. B. (2004). Frontal EEG asymmetry as a moderator and mediator of emotion. Biological Psychology, 67, 7-49. doi: 10.1016/j.biopsycho.2004.03.002

Coan, J. A., Allen, J. J. B., \& Harmon-Jones, E. (2001). Voluntary facial expression and hemispheric asymmetry over the frontal cortex. Psychophysiology, 38, 912-925. doi: $10.1111 / 1469-8986.3860912$

Coan, J. A., Allen, J. J. B., \& McKnight, P. E. (2006). A capability model of individual differences in frontal EEG asymmetry. Biological Psychology, 72, 198-207. doi: 10.1016/j.biopsycho.2005.10.003

Cohen, M. X. (2011). It's about time. Frontiers in Human Neuroscience, 5, 16. doi: 10.3389/fnhum.2011.00002

Craig, A. D. (2005). Forebrain emotional asymmetry: A neuroanatomical basis? Trends in Cognitive Sciences, 9, 566-571.

Curtis, C. E., \& D'Esposito, M. (2003). Persistent activity in the prefrontal cortex during working memory. Trends in Cognitive Sciences, 7, 415-423. doi: 10.1016/s13646613(03)00197-9

Curtis, W. J., \& Cicchetti, D. (2007). Emotion and resilience: A multilevel investigation of hemispheric electroencephalogram asymmetry and emotion regulation in maltreated and nonmaltreated children. Development and Psychopathology, 19, 811-840. doi: $10.1017 / \mathrm{s} 0954579407000405$

Davidson, R. J. (1998a). Affective style and affective disorders: Perspectives from affective neuroscience. Cognition \& Emotion, 12, 307-330. 
Davidson, R. J. (1998b). Anterior electrophysiological asymmetries, emotion, and depression: Conceptual and methodological conundrums. Psychophysiology, 35, 607-614. doi: $10.1017 / \mathrm{s} 0048577298000134$

Davidson, R. J. (2000). Affective style, psychopathology, and resilience: Brain mechanisms and plasticity. American Psychologist, 55, 1196-1214.

Davidson, R. J. (2004). What does the prefrontal cortex "do" in affect: Perspectives on frontal EEG asymmetry research. Biological Psychology, 67, 219-233. doi: 10.1016/j.biopsycho.2004.03.008

Davidson, R. J., \& Irwin, W. (1999). The functional neuroanatomy of emotion and affective style. Trends in Cognitive Sciences, 3, 11-21. doi: 10.1016/s1364-6613(98)01265-0

Davidson, R. J., Marshall, J. R., Tomarken, A. J., \& Henriques, J. B. (2000). While a phobic waits: Regional brain electrical and autonomic activity in social phobics during anticipation of public speaking. Biological Psychiatry, 47, 85-95.

Davis, M., Walker, D. L., \& Lee, Y. L. (1997). Roles of the amygdala and bed nucleus of the stria terminalis in fear and anxiety measured with the acoustic startle reflex - Possible relevance to PTSD. Annals of the New York Academy of Sciences, 821, 305-331. doi: 10.1111/j.1749-6632.1997.tb48289.x

De Jong, P. J., Merckelbach, H., \& Nijman, H. (1995). Hemisphere preference, anxiety, and covariation bias. Personality and Individual Differences, 18, 363-371. doi: 10.1016/0191-8869(94)00173-p

de Munck, J. C., Goncalves, S. I., Mammoliti, R., Heethaar, R. M., \& da Silva, F. H. L. (2009). Interactions between different EEG frequency bands and their effect on alphafMRI correlations. Neuroimage, 47, 69-76. doi: 10.1016/j.neuroimage.2009.04.029

Depue, B. E., Curran, T., \& Banich, M. T. (2007). Prefrontal regions orchestrate suppression of emotional memories via a two-phase process. Science, 317, 215-219. doi: 10.1126/science. 1139560

Dolan, R. J., \& Fletcher, P. C. (1997). Dissociating prefrontal and hippocampal function in episodic memory encoding. Nature, 388, 582-585.

Dolcos, F., Iordan, A. D., Kragel, J., Stokes, J., Campbell, R., McCarthy, G., \& Cabeza, R. (2013). Neural correlates of opposing effects of emotional distraction on working memory and episodic memory: an event-related FMRI investigation. Frontiers in Psychology, 4. 
Drabant, E. M., McRae, K., Manuck, S. B., Hariri, A. R., \& Gross, J. J. (2009). Individual differences in typical reappraisal use predict amygdala and prefrontal responses. Biological Psychiatry, 65, 367-373. doi: 10.1016/j.biopsych.2008.09.007

Duncan, J., \& Owen, A. M. (2000). Common regions of the human frontal lobe recruited by diverse cognitive demands. Trends in Neurosciences, 23, 475-483.

Dyck, M., Loughead, J., Kellermann, T., Boers, F., Gur, R. C., \& Mathiak, K. (2011). Cognitive versus automatic mechanisms of mood induction differentially activate left and right amygdala. Neuroimage, 54, 2503-2513. doi: 10.1016/j.neuroimage.2010.10.013

Ehlers, A. (2010). Understanding and treating unwanted trauma memories in posttraumatic stress disorder. Zeitschrift Fur Psychologie-Journal of Psychology, 218, 141-145. doi: 10.1027/0044-3409/a000021

Ekman, P., \& Davidson, R. J. (1993). Voluntary smiling changes regional brain activity. Psychological Science, 4, 342-345. doi: 10.1111/j.1467-9280.1993.tb00576.x

Epstein, C. M., Sekino, M., Yamaguchi, K., Kamiya, S., \& Ueno, S. (2002). Asymmetries of prefrontal cortex in human episodic memory: effects of transcranial magnetic stimulation on learning abstract patterns. Neuroscience Letters, 320, 5-8. doi: 10.1016/s0304-3940(01)02573-3

Etkin, A., \& Wager, T. D. (2007). Functional neuroimaging of anxiety: A meta-analysis of emotional processing in PTSD, social anxiety disorder, and specific phobia. American Journal of Psychiatry, 164, 1476-1488. doi: 10.1176/appi.ajp.2007.07030504

Falconer, E., Bryant, R., Felmingham, K. L., Kemp, A. H., Gordon, E., Peduto, A., . . . Williams, L. M. (2008). The neural networks of inhibitory control in posttraumatic stress disorder. Journal of Psychiatry and Neuroscience, 33, 413-422.

Fani, N., Jovanovic, T., Ely, T. D., Bradley, B., Gutman, D., Tone, E. B., \& Ressler, K. J. (2012). Neural correlates of attention bias to threat in post-traumatic stress disorder. Biological Psychology, 90, 134-142.

Fletcher, P. C., \& Henson, R. N. A. (2001). Frontal lobes and human memory - Insights from functional neuroimaging. Brain, 124, 849-881. doi: 10.1093/brain/124.5.849

Fletcher, P. C., Shallice, T., \& Dolan, R. J. (1998). The functional roles of prefrontal cortex in episodic memory - I. Encoding. Brain, 121, 1239-1248.

Forbes, D., Elhai, J. D., Lockwood, E., Creamer, M., Frueh, B. C., \& Magruder, K. M. (2012). The structure of posttraumatic psychopathology in veterans attending primary care. Journal of Anxiety Disorders, 26, 95-101. doi: 10.1016/j.janxdis.2011.09.004 
Foster, P. S., \& Harrison, D. W. (2006). Magnitude of cerebral asymmetry at rest: Covariation with baseline cardiovascular activity. Brain and Cognition, 61, 286-297. doi: 10.1016/j.bandc.2006.02.004

Francati, V., Vermetten, E., \& Bremner, J. D. (2007). Functional neuroimaging studies in posttraumatic stress disorder: Review of current methods and findings. Depression and Anxiety, 24, 202-218. doi: 10.1002/da.20208

Friedman, M. J., Resick, P. A., Bryant, R. A., \& Brewin, C. R. (2011). Considering PTSD for DSM-5. Depression and Anxiety, 28, 750-769.

Frueh, B. C., Hamner, M. B., Cahill, S. P., Gold, P. B., \& Hamlin, K. L. (2000). Apparent symptom overreporting in combat veterans evaluated for PTSD. Clinical Psychology Review, 20, 853-885. doi: 10.1016/s0272-7358(99)00015-x

Gable, P., \& Harmon-Jones, E. (2008). Relative left frontal activation to appetitive stimuli: Considering the role of individual differences. Psychophysiology, 45, 275-278. doi: 10.1111/j.1469-8986.2007.00627.x

Gerhards, F., Yehuda, R., Shoham, M., \& Hellhammer, D. H. (1997). Abnormal cerebral laterality in posttraumatic stress disorder. Annals of the New York Academy of Sciences, 821, 482-485. doi: 10.1111/j.1749-6632.1997.tb48311.x

Gilboa, A. (2004). Autobiographical and episodic memory - one and the same? Evidence from prefrontal activation in neuroimaging studies. Neuropsychologia, 42, 1336-1349. doi: 10.1016/j.neuropsychologia.2004.02.014

Goodman, R. N., Rietschel, J. C., Lo, L.-C., Costanzo, M. E., \& Hatfield, B. D. (2013). Stress, emotion regulation and cognitive performance: The predictive contributions of trait and state relative frontal EEG alpha asymmetry. International Journal of Psychophysiology, 87, 115-123. doi: 10.1016/j.ijpsycho.2012.09.008

Gordon, E., Palmer, D. M., \& Cooper, N. (2010). EEG alpha asymmetry in schizophrenia, depression, PTSD, panic disorder, ADHD and conduct disorder. Clinical EEG and Neuroscience, 41, 178-183. doi: 10.1177/155005941004100404

Greenberg, D. L., Rice, H. J., Cooper, J. J., Cabeza, R., Rubin, D. C., \& LaBar, K. S. (2005). Co-activation of the amygdala, hippocampus and inferior frontal gyrus during autobiographical memory retrieval. Neuropsychologia, 43, 659-674. doi: 10.1016/j.neuropsychologia.2004.09.002

Grimm, A., Hulse, L., Preiss, M., \& Schmidt, S. (2012). Post- and peritraumatic stress in disaster survivors: An explorative study about the influence of individual and event 
characteristics across different types of disasters. European Journal of Psychotraumatology, 3. doi: 10.3402/ejpt.v3i0.7382

Grimm, S., Beck, J., Schuepbach, D., Hell, D., Boesiger, P., Bermpohl, F., . . Northoff, G. (2008). Imbalance between left and right dorsolateral prefrontal cortex in major depression is linked to negative emotional judgment: An fMRI study in severe major depressive disorder. Biological Psychiatry, 63, 369-376. doi: 10.1016/j.biopsych.2007.05.033

Grimm, S., Schmidt, C. F., Bermpohl, F., Heinzel, A., Dahlem, Y., Wyss, M., . . Northoff, G. (2006). Segregated neural representation of distinct emotion dimensions in the prefrontal cortex: An fMRI study. Neuroimage, 30, 325-340. doi: 10.1016/j.neuroimage.2005.09.006

Gross, J. J., \& Thompson, R. A. (2007). Emotion regulation: Conceptual foundations. In J. J. Gross (Ed.), Handbook of emotion regulation (pp. 3-24). New York: The Guilford Press.

Habib, R., Nyberg, L., \& Tulving, E. (2003). Hemispheric asymmetries of memory: The HERA model revisited. Trends in Cognitive Sciences, 7, 241-245. doi: 10.1016/s13646613(03)00110-4

Hagemann, D. (2004). Individual differences in anterior EEG asymmetry: Methodological problems and solutions. Biological Psychology, 67, 157-182. doi: 10.1016/j.biopsycho.2004.03.006

Hagemann, D., Hewig, J., Seifert, J., Naumann, E., \& Bartussek, D. (2005). The latent statetrait structure of resting EEG asymmetry: Replication and extension. Psychophysiology, 42, 740-752. doi: 10.1111/j.1469-8986.2005.00367.x

Hagemann, D., Naumann, E., \& Thayer, J. F. (2001). The quest for the EEG reference revisited: A glance from brain asymmetry research. Psychophysiology, 38, 847-857.

Hagemann, D., Naumann, E., Thayer, J. F., \& Bartussek, D. (2002). Does resting electroencephalograph asymmetry reflect a trait? An application of latent state-trait theory. Journal of Personality and Social Psychology, 82, 619-641. doi: $10.1037 / / 0022-3514.82 .4 .619$

Hagemann, D., Waldstein, S. R., \& Thayer, J. F. (2003). Central and autonomic nervous system integration in emotion. Brain and Cognition, 52, 79-87. doi: 10.1016/s02782626(03)00011-3 
Harmon-Jones, E. (2004). Contributions from research on anger and cognitive dissonance to understanding the motivational functions of asymmetrical frontal brain activity. Biological Psychology, 67, 51-76. doi: 10.1016/j.biopsycho.2004.03.003

Harmon-Jones, E. (2006). Unilateral right-hand contractions cause contralateral alpha power suppression and approach motivational affective experience. Psychophysiology, 43, 598-603. doi: 10.1111/j.1469-8986.2006.00465.x

Harmon-Jones, E., Gable, P. A., \& Peterson, C. K. (2010). The role of asymmetric frontal cortical activity in emotion-related phenomena: A review and update. Biological Psychology, 84, 451-462.

Heinzel, A., Bermpohl, F., Niese, R., Pfennig, A., Pascual-Leone, A., Schlaug, G., \& Northoff, G. (2005). How do we modulate our emotions? Parametric fMRI reveals cortical midline structures as regions specifically involved in the processing of emotional valences. Cognitive Brain Research, 25, 348-358. doi: 10.1016/j.cogbrainres.2005.06.009

Heller, W. (1993). Neuropsychological mechanisms of individual differences in emotion, personality, and arousal. Neuropsychology, 7, 476-489.

Heller, W., \& Nitschke, J. B. (1998). The puzzle of regional brain activity in depression and anxiety: The importance of subtypes and comorbidity. Cognition \& Emotion, 12, 421447.

Heller, W., Nitschke, J. B., Etienne, M. A., \& Miller, G. A. (1997). Patterns of regional brain activity differentiate types of anxiety. Journal of Abnormal Psychology, 106, 376-385. doi: 10.1037//0021-843x.106.3.376

Henson, R. N. A., Rugg, M. D., Shallice, T., \& Dolan, R. J. (2000). Confidence in recognition memory for words: Dissociating right prefrontal roles in episodic retrieval. Journal of Cognitive Neuroscience, 12, 913-923.

Herrington, J. D., Heller, W., Mohanty, A., Engels, A. S., Banich, M. T., Webb, A. G., \& Miller, G. A. (2010). Localization of asymmetric brain function in emotion and depression. Psychophysiology, 47, 442-454. doi: 10.1111/j.1469-8986.2009.00958.x

Inslicht, S. S., Otte, C., McCaslin, S. E., Apfel, B. A., Henn-Haase, C., Metzler, T., . . . Marmar, C. R. (2011). Cortisol awakening response prospectively predicts peritraumatic and acute stress reactions in police officers. Biological Psychiatry, 70, 1055-1062. 
Jaworska, N., Berrigan, L., Ahmed, A. G., Gray, J., Bradford, J., Korovessis, A., . . Knott, V. (2012). Resting electrocortical activity in adults with dysfunctional anger: A pilot study. Psychiatry Research: Neuroimaging, 203, 229-236.

Jensen, O., Bonnefond, M., \& VanRullen, R. (2012). An oscillatory mechanism for prioritizing salient unattended stimuli. Trends in Cognitive Sciences, 16, 200-206. doi: 10.1016/j.tics.2012.03.002

Johnsen, B. H., \& Hugdahl, K. (1993). Right-hemisphere representation of autonomic conditioning to facial emotional expressions. Psychophysiology, 30, 274-278. doi: 10.1111/j.1469-8986.1993.tb03353.x

Kagan, J., \& Snidman, N. (1999). Early childhood predictors of adult anxiety disorders. Biological Psychiatry, 46, 1536-1541. doi: 10.1016/s0006-3223(99)00137-7

Kemp, A. H., Griffiths, K., Felmingham, K. L., Shankman, S. A., Drinkenburg, W., Arns, M., ... Bryant, R. A. (2010). Disorder specificity despite comorbidity: Resting EEG alpha asymmetry in major depressive disorder and post-traumatic stress disorder. Biological Psychology, 85, 350-354. doi: 10.1016/j.biopsycho.2010.08.001

Kensinger, E. A., \& Choi, E. S. (2009). When side matters: Hemispheric processing and the visual specificity of emotional memories. Journal of Experimental PsychologyLearning Memory and Cognition, 35, 247-253. doi: 10.1037/a0013414

Kessler, R. C., Sonnega, A., Bromet, E., Hughes, M., \& Nelson, C. B. (1995). Posttraumatic stress disorder in the National Comorbidity Survey. Archives of General Psychiatry, $52,1048-1060$.

Kilpatrick, D. G., Resnick, H. S., \& Acierno, R. (2009). Should PTSD criterion A be retained? Journal of Traumatic Stress, 22, 374-383.

Kilpatrick, L., \& Cahill, L. (2003). Amygdala modulation of parahippocampal and frontal regions during emotionally influenced memory storage. NeuroImage, 20, 2091-2099. doi: 10.1016/j.neuroimage.2003.08.006

Kindt, M., van den Hout, M., Arntz, A., \& Drost, J. (2008). The influence of data-driven versus conceptually-driven processing on the development of PTSD-like symptoms. Journal of Behavior Therapy and Experimental Psychiatry, 39, 546-557. doi: 10.1016/j.jbtep.2007.12.003

King, J. A., Hartley, T., Spiers, H. J., Maguire, E. A., \& Burgess, N. (2005). Anterior prefrontal involvement in episodic retrieval reflects contextual interference. NeuroImage, 28, 256-267. doi: 10.1016/j.neuroimage.2005.05.057 
Klimesch, W. (1999). EEG alpha and theta oscillations reflect cognitive and memory performance: A review and analysis. Brain Research Reviews, 29, 169-195. doi: 10.1016/s0165-0173(98)00056-3

Klimesch, W., Sauseng, P., \& Hanslmayr, S. (2007). EEG alpha oscillations: The inhibitiontiming hypothesis. Brain Research Reviews, 53, 63-88. doi: 10.1016/j.brainresrev.2006.06.003

Kober, H., Barrett, L. F., Joseph, J., Bliss-Moreau, E., Lindquist, K., \& Wager, T. D. (2008). Functional grouping and cortical-subcortical interactions in emotion: A meta-analysis of neuroimaging studies. NeuroImage, 42, 998-1031. doi: 10.1016/j.neuroimage.2008.03.059

Koenigs, M., \& Grafman, J. (2009). Posttraumatic stress disorder: The role of medial prefrontal cortex and amygdala. Neuroscientist, 15, 540-548. doi: $10.1177 / 1073858409333072$

Kompus, K., Hugdahl, K., Öhman, A., Marklund, P., \& Nyberg, L. (2009). Distinct control networks for cognition and emotion in the prefrontal cortex. Neuroscience Letters, 467, 76-80. doi: 10.1016/j.neulet.2009.10.005

Koslov, K., Mendes, W. B., Pajtas, P. E., \& Pizzagalli, D. A. (2011). Asymmetry in resting intracortical activity as a buffer to social threat. Psychological Science, 22, 641-649. doi: $10.1177 / 0956797611403156$

Kurchakova, M. S., Tarabrina, N. V., Illarionova, M. D., \& Grishkova, O. S. (2009). Correlation of evoked potentials indices with characteristics of traumatic stress in combatant. Psikhologicheskii Zhurnal, 30, 96-106.

Lang, P. J., Bradley, M. M., \& Cuthbert, B. N. (2005). International Affective Picture System (IAPS): Instruction manual and affective ratings. Technical report A-6. Gainesville, FL: University of Florida.

Lanius, R. A., Bluhm, R. L., Coupland, N. J., Hegadoren, K. M., Rowe, B., Théberge, J., . . . Brimson, M. (2010). Default mode network connectivity as a predictor of posttraumatic stress disorder symptom severity in acutely traumatized subjects. Acta Psychiatrica Scandinavica, 121, 33-40. doi: 10.1111/j.1600-0447.2009.01391.x

Lehrner, A., \& Yehuda, R. (2014). Biomarkers of PTSD: Military applications and considerations. European Journal of Psychotraumatology, 5. doi: 10.3402/ejpt.v5.23797

Leyman, L., De Raedt, R., Vanderhasselt, M. A., \& Baeken, C. (2009). Influence of highfrequency repetitive transcranial magnetic stimulation over the dorsolateral prefrontal 
cortex on the inhibition of emotional information in healthy volunteers. Psychological Medicine, 39, 1019-1028. doi: 10.1017/s0033291708004431

Linden, M., Baumann, K., Rotter, M., \& Schippan, B. (2008). Posttraumatic embitterment disorder in comparison to other mental disorders. Psychotherapy and Psychosomatics, 77, 50-56. doi: 10.1159/000110060

Liu, H. S., Stufflebeam, S. M., Sepulcre, J., Hedden, T., \& Buckner, R. L. (2009). Evidence from intrinsic activity that asymmetry of the human brain is controlled by multiple factors. Proceedings of the National Academy of Sciences of the United States of America, 106, 20499-20503. doi: 10.1073/pnas.0908073106

Lommen, M. J. J., Engelhard, I. M., Sijbrandij, M., van den Hout, M. A., \& Hermans, D. (2013). Pre-trauma individual differences in extinction learning predict posttraumatic stress. Behaviour Research and Therapy, 51, 63-67. doi: 10.1016/j.brat.2012.11.004

Lopez-Duran, N. L., Nusslock, R., George, C., \& Kovacs, M. (2012). Frontal EEG asymmetry moderates the effects of stressful life events on internalizing symptoms in children at familial risk for depression. Psychophysiology, 49, 510-521. doi: 10.1111/j.14698986.2011.01332.x

Maier, S. F., \& Watkins, L. R. (2010). Role of the medial prefrontal cortex in coping and resilience. Brain Research, 1355, 52-60. doi: 10.1016/j.brainres.2010.08.039

Manenti, R., Cotelli, M., Calabria, M., Maioli, C., \& Miniussi, C. (2010). Ther role of the dorsolateral prefrontal cortex in retrieval from long-term memory depends on strategies: A repetitive transcranial magnetic stimulation study. Neuroscience, 166, 501-507. doi: 10.1016/j.neuroscience.2009.12.037

Marsolek, C. J. (1999). Dissociable neural, subsystems underlie abstract and specific object recognition. Psychological Science, 10, 111-118.

Marsolek, C. J., \& Burgund, E. D. (2008). Dissociable neural subsystems underlie visual working memory for abstract categories and specific exemplars. Cognitive, Affective, \& Behavioral Neuroscience, 8, 17-24. doi: 10.3758/cabn.8.1.17

Mathersul, D., Williams, L. M., Hopkinson, P. J., \& Kemp, A. H. (2008). Investigating models of affect: Relationships among EEG alpha asymmetry, depression, and anxiety. Emotion, 8, 560-572. doi: 10.1037/a0012811

McCaffrey, R. J., Lorig, T. S., Pendrey, D. L., McCutcheon, N. B., \& Garrett, J. C. (1993). Odor-induced EEG changes in PTSD Vietnam veterans. Journal of Traumatic Stress, 6, 213-224. 
McHugh, T., Forbes, D., Bates, G., Hopwood, M., \& Creamer, M. (2012). Anger in PTSD: Is there a need for a concept of PTSD-related posttraumatic anger? Clinical Psychology Review, 32, 93-104. doi: 10.1016/j.cpr.2011.07.013

McMenamin, B., \& Marsolek, C. (2013). Can theories of visual representation help to explain asymmetries in amygdala function? Cognitive, Affective, \& Behavioral Neuroscience, 13, 211-224. doi: 10.3758/s13415-012-0139-1

McNally, R. J. (2006). Cognitive abnormalities in post-traumatic stress disorder. Trends in Cognitive Sciences, 10, 271-277. doi: 10.1016/j.tics.2006.04.007

McNally, R. J., \& Robinaugh, D. J. (2011). Risk factors and posttraumatic stress disorder: Are they especially predictive following exposure to less severe stressors? Depression and Anxiety, 28, 1091-1096. doi: 10.1002/da.20867

Merckelbach, H., Dijkstra, A., De Jong, P. J., \& Muris, P. (1994). No effects of verbal versus imaginal cognitive strategies on emotional responses to aversive slides. Behavioural and Cognitive Psychotherapy, 22, 199-209.

Merckelbach, H., Muris, P., Pool, K., De Jong, P. J., \& Schouten, E. (1996). Reliability and validity of a paper-and-pencil test measuring hemisphere preference. European Journal of Personality, 10, 221-231. doi: 10.1002/(sici)10990984(199609)10:3<221::aid-per253>3.0.co;2-9

Metzger, L. J., Paige, S. R., Carson, M. A., Lasko, N. B., Paulus, L. A., Pitman, R. K., \& Orr, S. P. (2004). PTSD arousal and depression symptoms associated with increased rightsided parietal EEG asymmetry. Journal of Abnormal Psychology, 113, 324-329. doi: 10.1037/0021-843x.113.2.324

Mikolajczak, M., Bodarwe, K., Laloyaux, O., Hansenne, M., \& Nelis, D. (2010). Association between frontal EEG asymmetries and emotional intelligence among adults. Personality and Individual Differences, 48, 177-181. doi: 10.1016/j.paid.2009.10.001

Miller, G. A., Crocker, L. D., Spielberg, J. M., Infantolino, Z. P., \& Heller, W. (2013). Issues in localization of brain function: The case of lateralized frontal cortex in cognition, emotion, and psychopathology. Frontiers in Integrative Neuroscience, 7. doi: 10.3389/fnint.2013.00002

Moores, K. A., Clark, C. R., McFarlane, A. C., Brown, G. C., Puce, A., \& Taylor, D. J. (2008). Abnormal recruitment of working memory updating networks during maintenance of trauma-neutral information in post-traumatic stress disorder. Psychiatry Research: Neuroimaging, 163, 156-170. 
Moscovitch, D. A., Santesso, D. L., Miskovic, V., McCabe, R. E., Antony, M. M., \& Schmidt, L. A. (2011). Frontal EEG asymmetry and symptom response to cognitive behavioral therapy in patients with social anxiety disorder. Biological Psychology, 87, 379-385.

Murphy, F. C., Nimmo-Smith, I., \& Lawrence, A. D. (2003). Functional neuroanatomy of emotion: A meta-analysis. Cognitive, Affective, \& Behavioral Neuroscience, 3, 207233.

Niendam, T., Laird, A., Ray, K., Dean, Y., Glahn, D., \& Carter, C. (2012). Meta-analytic evidence for a superordinate cognitive control network subserving diverse executive functions. Cognitive, Affective, \& Behavioral Neuroscience, 12, 241-268. doi: 10.3758/s 13415-011-0083-5

Nitschke, J. B., Heller, W., Etienne, M. A., \& Miller, G. A. (2004). Prefrontal cortex activity differentiates processes affecting memory in depression. Biological Psychology, 67, 125-143. doi: 10.1016/j.biopsycho.2004.03.004

Northoff, G., Heinzel, A., Bermpohl, F., Niese, R., Pfennig, A., Pascual-Leone, A., \& Schlaug, G. (2004). Reciprocal modulation and attenuation in the prefrontal cortex: An fMRI study on emotional-cognitive interaction. Human Brain Mapping, 21, 202212. doi: $10.1002 / \mathrm{hbm} .20002$

Northoff, G., Heinzel, A., de Greck, M., Bermpohl, F., Dobrowolny, H., \& Panksepp, J. (2006). Self-referential processing in our brain: A meta-analysis of imaging studies on the self. Neuroimage, 31, 440-457. doi: 10.1016/j.neuroimage.2005.12.002

Nusslock, R., Shackman, A. J., Harmon-Jones, E., Alloy, L. B., Coan, J. A., \& Abramson, L. Y. (2011). Cognitive vulnerability and frontal brain asymmetry: Common predictors of first prospective depressive episode. Journal of Abnormal Psychology, 120, $497-$ 503. doi: $10.1037 / \mathrm{a} 0022940$

Ochsner, K. N., Bunge, S. A., Gross, J. J., \& Gabrieli, J. D. E. (2002). Rethinking feelings: An fMRI study of the cognitive regulation of emotion. Journal of Cognitive Neuroscience, 14, 1215-1229.

Ochsner, K. N., \& Gross, J. J. (2005). The cognitive control of emotion. Trends in Cognitive Sciences, 9, 242-249. doi: 10.1016/j.tics.2005.03.010

Orr, S. P., \& Roth, W. T. (2000). Psychophysiological assessment: Clinical applications for PTSD. Journal of Affective Disorders, 61, 225-240. 
Osuch, E. A., Benson, B. E., Luckenbaugh, D. A., Geraci, M., Post, R. M., \& McCann, U. (2009). Repetitive TMS combined with exposure therapy for PTSD: A preliminary study. Journal of Anxiety Disorders, 23, 54-59. doi: 10.1016/j.janxdis.2008.03.015

Pallanti, S., \& Bernardi, S. (2009). Neurobiology of repeated transcranial magnetic stimulation in the treatment of anxiety: A critical review. International Clinical Psychopharmacology, 24, 163-173. doi: 10.1097/YIC.0b013e32832c2639

Papousek, I., Schulter, G., \& Lang, B. (2009). Effects of emotionally contagious films on changes in hemisphere-specific cognitive performance. Emotion, 9, 510-519. doi: $10.1037 / \mathrm{a} 0016299$

Parvaz, M., MacNamara, A., Goldstein, R., \& Hajcak, G. (2012). Event-related induced frontal alpha as a marker of lateral prefrontal cortex activation during cognitive reappraisal. Cognitive, Affective, \& Behavioral Neuroscience, 12, 730-740. doi: 10.3758/s13415-012-0107-9

Patel, R., Spreng, R. N., Shin, L. M., \& Girard, T. A. (2012). Neurocircuitry models of posttraumatic stress disorder and beyond: a meta-analysis of functional neuroimaging studies. Neuroscience \& Biobehavioral Reviews, 36, 2130-2142. doi: 10.1016/j.neubiorev.2012.06.003

Peeters, F., Ronner, J., Bodar, L., van Os, J., \& Lousberg, R. (2014). Validation of a neurofeedback paradigm: Manipulating frontal EEG alpha-activity and its impact on mood. International Journal of Psychophysiology, 93, 116-120. doi: 10.1016/j.ijpsycho.2013.06.010

Peres, J. F. P., Foerster, B., Santana, L. G., Fereira, M. D., Nasello, A. G., Savoia, M., . . . Lederman, H. (2011). Police officers under attack: Resilience implications of an fMRI study. Journal of Psychiatric Research, 45, 727-734. doi: 10.1016/j.jpsychires.2010.11.004

Pfurtscheller, G., Stancak, A., \& Neuper, C. (1996). Event-related synchronization (ERS) in the alpha band - An electrophysiological correlate of cortical idling: A review. International Journal of Psychophysiology, 24, 39-46. doi: 10.1016/s01678760(96)00066-9

Phillips, M., Ladouceur, C., \& Drevets, W. (2008). A neural model of voluntary and automatic emotion regulation: Implications for understanding the pathophysiology and neurodevelopment of bipolar disorder. Molecular Psychiatry, 13, 833-857. doi: 10.1038/mp.2008.65 
Pizzagalli, D. A., Sherwood, R. J., Henriques, J. B., \& Davidson, R. J. (2005). Frontal brain asymmetry and reward responsiveness - A source-localization study. Psychological Science, 16, 805-813.

Pole, N. (2007). The psychophysiology of posttraumatic stress disorder: A meta-analysis. Psychological Bulletin, 133, 725.

Pole, N., Neylan, T. C., Otte, C., Henn-Hasse, C., Metzler, T. J., \& Marmar, C. R. (2009). Prospective prediction of posttraumatic stress disorder symptoms using fear potentiated auditory startle responses. Biological Psychiatry, 65, 235-240. doi: 10.1016/j.biopsych.2008.07.015

Quaedflieg, C. W. E. M., Meyer, T., Smulders, F., \& Smeets, T. (2015). The functional role of individual-alpha based frontal asymmetry in stress responding. Biological Psychology, 104, 75-81. doi: 10.1016/j.biopsycho.2014.11.014

Rabe, S., Beauducel, A., Zöllner, T., Maercker, A., \& Karl, A. (2006). Regional brain electrical activity in posttraumatic stress disorder after motor vehicle accident. Journal of Abnormal Psychology, 115, 687-698. doi: 10.1037/0021-843x.115.4.687

Rabe, S., Zöllner, T., Beauducel, A., Maercker, A., \& Karl, A. (2008). Changes in brain electrical activity after cognitive behavioral therapy for posttraumatic stress disorder in patients injured in motor vehicle accidents. Psychosomatic Medicine, 70, 13-19. doi: 10.1097/PSY.0b013e31815aa325

Rabe, S., Zöllner, T., Maercker, A., \& Karl, A. (2006). Neural correlates of posttraumatic growth after severe motor vehicle accidents. Journal of Consulting and Clinical Psychology, 74, 880-886. doi: 10.1037/0022-006x.74.5.880

Reid, S. A., Duke, L. M., \& Allen, J. J. B. (1998). Resting frontal electroencephalographic asymmetry in depression: Inconsistencies suggest the need to identify mediating factors. Psychophysiology, 35, 389-404.

Rosen, G. M., \& Lilienfeld, S. O. (2008). Posttraumatic stress disorder: An empirical evaluation of core assumptions. Clinical Psychology Review, 28, 837-868. doi: 10.1016/j.cpr.2007.12.002

Rossi, S., Innocenti, I., Polizzotto, N. R., Feurra, M., DeCapua, A., Ulivelli, M., . . Cappa, S. F. (2011). Temporal dynamics of memory trace formation in the human prefrontal cortex. Cerebral Cortex, 21, 368-373.

Rotenberg, V. S. (2004). The peculiarity of the right-hemisphere function in depression: Solving the paradoxes. Progress in Neuro-Psychopharmacology \& Biological Psychiatry, 28, 1-13. doi: 10.1016/s0278-5846(03)00163-5 
Sandrini, M., Cappa, S. F., Rossi, S., Rossini, P. M., \& Miniussi, C. (2003). The role of prefrontal cortex in verbal episodic memory: rTMS evidence. Journal of Cognitive Neuroscience, 15, 855-861. doi: 10.1162/089892903322370771

Sandrini, M., Censor, N., Mishoe, J., \& Cohen, Leonardo G. (2013). Causal role of prefrontal cortex in strengthening of episodic memories through reconsolidation. Current Biology, 23, 2181-2184. doi: 10.1016/j.cub.2013.08.045

Scheeringa, R., Petersson, K. M., Kleinschmidt, A., Jensen, O., \& Bastiaansen, M. C. M. (2012). EEG alpha power modulation of fMRI resting state connectivity. Brain Connectivity, 2, 254-264. doi: 10.1089/brain.2012.0088

Schmidt, L. A., Miskovic, V., Boyle, M., \& Saigal, S. (2010). Frontal electroencephalogram asymmetry, salivary cortisol, and internalizing behavior problems in young adults who were born at extremely low birth weight. Child Development, 81, 183-199.

Schmidt, U., Kaltwasser, S. F., \& Wotjak, C. T. (2013). Biomarkers in posttraumatic stress disorder: Overview and implications for future research. Disease Markers, 35, 43-54. doi: $10.1155 / 2013 / 835876$

Schore, A. N. (2002). Dysregulation of the right brain: A fundamental mechanism of traumatic attachment and the psychopathogenesis of posttraumatic stress disorder. Australian and New Zealand Journal of Psychiatry, 36, 9-30. doi: 10.1046/j.14401614.2002.00996.x

Schutter, D. J. L. G., \& Harmon-Jones, E. (2013). The corpus callosum: A commissural road to anger and aggression. Neuroscience \& Biobehavioral Reviews, 37, 2481-2488. doi: 10.1016/j.neubiorev.2013.07.013

Segrave, R. A., Cooper, N. R., Thomson, R. H., Croft, R. J., Sheppard, D. M., \& Fitzgerald, P. B. (2011). Individualized alpha activity and frontal asymmetry in major depression. Clinical EEG and Neuroscience, 42, 45-52.

Shankman, S. A., Silverstein, S. M., Williams, L. M., Hopkinson, P. J., Kemp, A. H., Felmingham, K. L., . . Clark, C. R. (2008). Resting electroencephalogram asymmetry and posttraumatic stress disorder. Journal of Traumatic Stress, 21, 190-198. doi: $10.1002 /$ jts.20319

Shin, L. M., Rauch, S. L., \& Pitman, R. K. (2006). Amygdala, medial prefrontal cortex, and hippocampal function in PTSD. In R. Yehuda (Ed.), Psychobiology of posttraumatic stress disorder: A decade of progress (Vol. 1071, pp. 67-79). Boston: New York Academy of Science. 
Simon-Thomas, E. R., Role, K. O., \& Knight, R. T. (2005). Behavioral and electrophysiological evidence of a right hemisphere bias for the influence of negative emotion on higher cognition. Journal of Cognitive Neuroscience, 17, 518-529.

Spielberg, J. M., Heller, W., \& Miller, G. A. (2013). Hierarchical brain networks active in approach and avoidance goal pursuit. Frontiers in Human Neuroscience, 7. doi: 284

10.3389/fnhum.2013.00284

Spielberg, J. M., Miller, G. A., Engels, A. S., Herrington, J. D., Sutton, B. P., Banich, M. T., \& Heller, W. (2011). Trait approach and avoidance motivation: Lateralized neural activity associated with executive function. NeuroImage, 54, 661-670. doi: 10.1016/j.neuroimage.2010.08.037

Spielberg, J. M., Miller, G. A., Warren, S. L., Engels, A. S., Crocker, L. D., Sutton, B. P., \& Heller, W. (2012). Trait motivation moderates neural activation associated with goal pursuit. Cognitive, Affective, \& Behavioral Neuroscience, 12, 308-322. doi: $10.3758 / \mathrm{s} 13415-012-0088-8$

Spivak, B., Segal, M., Mester, R., \& Weizman, A. (1998). Lateral preference in posttraumatic stress disorder. Psychological Medicine, 28, 229-232. doi: $10.1017 / \mathrm{s} 0033291797005837$

St Jacques, P. L., Botzung, A., Miles, A., \& Rubin, D. C. (2011). Functional neuroimaging of emotionally intense autobiographical memories in post-traumatic stress disorder. Journal of Psychiatric Research, 45, 630-637. doi: 10.1016/j.jpsychires.2010.10.011

Stewart, J. L., Bismark, A. W., Towers, D. N., Coan, J. A., \& Allen, J. J. B. (2010). Resting frontal EEG asymmetry as an endophenotype for depression risk: Sex-specific patterns of frontal brain asymmetry. Journal of Abnormal Psychology, 119, 502-512. doi: $10.1037 / \mathrm{a} 0019196$

Stewart, J. L., Coan, J. A., Towers, D. N., \& Allen, J. J. (2014). Resting and task-elicited prefrontal EEG alpha asymmetry in depression: Support for the capability model. Psychophysiology. doi: 10.1111/psyp.12191

Stewart, J. L., Coan, J. A., Towers, D. N., \& Allen, J. J. B. (2011). Frontal EEG asymmetry during emotional challenge differentiates individuals with and without lifetime major depressive disorder. Journal of Affective Disorders, 129, 167-174. doi: 10.1016/j.jad.2010.08.029

Sullivan, R. M., \& Gratton, A. (2002). Prefrontal cortical regulation of hypothalamicpituitary-adrenal function in the rat and implications for psychopathology: Side 
matters. Psychoneuroendocrinology, 27, 99-114. doi: 10.1016/s0306-4530(01)000385

Sutton, S. K., \& Davidson, R. J. (1997). Prefrontal brain asymmetry: A biological substrate of the behavioral approach and inhibition systems. Psychological Science, 8, 204-210. doi: 10.1111/j.1467-9280.1997.tb00413.x

Suvak, M. K., \& Barrett, L. F. (2011). Considering PTSD from the perspective of brain processes: A psychological construction approach. Journal of Traumatic Stress, 24, 324. doi: $10.1002 /$ jts.20618

Svoboda, E., McKinnon, M. C., \& Levine, B. (2006). The functional neuroanatomy of autobiographical memory: A meta-analysis. Neuropsychologia, 44, 2189-2208. doi: 10.1016/j.neuropsychologia.2006.05.023

Teneback, C. C., Nahas, Z., Speer, A. M., Molloy, M., Stallings, L. E., Spicer, K. M., . . . George, M. S. (1999). Changes in prefrontal cortex and paralimbic activity in depression following two weeks of daily left prefrontal TMS. Journal of Neuropsychiatry and Clinical Neurosciences, 11, 426-435.

Tenke, C. E., \& Kayser, J. (2005). Reference-free quantification of EEG spectra: Combining current source density (CSD) and frequency principal components analysis (fPCA). Clinical Neurophysiology, 116, 2826-2846. doi: 10.1016/j.clinph.2005.08.007

Thayer, J. F., Ahs, F., Fredrikson, M., Sollers, J. J., \& Wager, T. D. (2012). A meta-analysis of heart rate variability and neuroimaging studies: Implications for heart rate variability as a marker of stress and health. Neuroscience \& Biobehavioral Reviews, 36, 747-756. doi: 10.1016/j.neubiorev.2011.11.009

Thibodeau, R., Jorgensen, R. S., \& Kim, S. (2006). Depression, anxiety, and resting frontal EEG asymmetry: A meta-analytic review. Journal of Abnormal Psychology, 115, 715729. doi: 10.1037/0021-843x.115.4.715

Tillman, G. D., Kimbrell, T. A., Calley, C. S., Kraut, M. A., Freeman, T. W., \& Hart, J. (2011). Repetitive transcranial magnetic stimulation and threat memory: Selective reduction of combat threat memory $\mathrm{P} 300$ response after right frontal-lobe stimulation. Journal of Neuropsychiatry and Clinical Neurosciences, 23, 40-47. doi: 10.1176/appi.neuropsych.23.1.40

Tomarken, A. J., Davidson, R. J., Wheeler, R. E., \& Doss, R. C. (1992). Individual differences in anterior brain asymmetry and fundamental dimensions of emotion. Journal of Personality and Social Psychology, 62, 676-687. 
Tomarken, A. J., Davidson, R. J., Wheeler, R. E., \& Kinney, L. (1992). Psychometric properties of resting anterior EEG asymmetry: Temporal stability and internal consistency. Psychophysiology, 29, 576-592.

Tomarken, A. J., Dichter, G. S., Garber, J., \& Simien, C. (2004). Resting frontal brain activity: linkages to maternal depression and socio-economic status among adolescents. Biological Psychology, 67, 77-102. doi: 10.1016/j.biopsycho.2004.03.011

Towers, D. N., \& Allen, J. J. B. (2009). A better estimate of the internal consistency reliability of frontal EEG asymmetry scores. Psychophysiology, 46, 132-142. doi: 10.1111/j.1469-8986.2008.00759.x

Tucker, D. M., \& Newman, J. P. (1981). Verbal versus imaginal cognitive strategies in the inhibition of emotional arousal. Cognitive Therapy and Research, 5, 197-202. doi: $10.1007 / \mathrm{bf} 01172527$

Turriziani, P., Smirni, D., Zappala, G., Mangano, G. R., Oliveri, M., \& Cipolotti, L. (2012). Enhancing memory performance with rTMS in healthy subjects and individuals with Mild Cognitive Impairment: the role of the right dorsolateral prefrontal cortex. Frontiers in Human Neuroscience, 6. doi: 10.3389/fnhum.2012.00062

Urry, H. L., Nitschke, J. B., Dolski, I., Jackson, D. C., Dalton, K. M., Mueller, C. J., . . . Davidson, R. J. (2004). Making a life worth living - Neural correlates of well-being. Psychological Science, 15, 367-372.

Van Praag, H. M., Asnis, G. M., Kahn, R. S., Brown, S. L., Korn, M., Friedman, J. M. H., \& Wetzler, S. (1990). Nosological tunnel vision in biological psychiatry: A plea for a functional psychopathology. Annals of the New York Academy of Sciences, 600, 501510. doi: 10.1111/j.1749-6632.1990.tb16905.x

van Wingen, G. A., Geuze, E., Vermetten, E., \& Fernandez, G. (2011). Perceived threat predicts the neural sequelae of combat stress. Molecular Psychiatry, 16, 664-671.

van Zuiden, M., Geuze, E., Willemen, H. L. D. M., Vermetten, E., Maas, M., Amarouchi, K., . . Heijnen, C. J. (2012). Glucocorticoid receptor pathway components predict posttraumatic stress disorder symptom development: A prospective study. Biological Psychiatry, 71, 309-316. doi: 10.1016/j.biopsych.2011.10.026

Vanderhasselt, M. A., Baeken, C., Hendricks, M., \& De Raedt, R. (2011). The effects of high frequency rTMS on negative attentional bias are influenced by baseline state anxiety. Neuropsychologia, 49, 1824-1830. doi: 10.1016/j.neuropsychologia.2011.03.006 
Vasterling, J. J., Duke, L. M., Tomlin, H., Lowery, N., \& Kaplan, E. (2004). Global-local visual processing in posttraumatic stress disorder. Journal of the International Neuropsychological Society, 10, 709-718. doi: 10.1017/s1355617704105031

Velo, J. R., Stewart, J. L., Hasler, B. P., Towers, D. N., \& Allen, J. J. B. (2012). Should it matter when we record? Time of year and time of day as factors influencing frontal EEG asymmetry. Biological Psychology, 91, 283-291.

Viinikainen, M., Jaaskelainen, I. R., Alexandrov, Y., Balk, M. H., Autti, T., \& Sams, M. (2010). Nonlinear relationship between emotional valence and brain activity: Evidence of separate negative and positive valence dimensions. Human Brain Mapping, 31, 1030-1040. doi: 10.1002/hbm.20915

Vogt, B. A., Finch, D. M., \& Olson, C. R. (1992). Functional heterogeneity in cingulate cortex: The anterior executive and posterior evaluative regions. Cerebral Cortex, 2, 435-443.

Vytal, K., Cornwell, B., Arkin, N., \& Grillon, C. (2012). Describing the interplay between anxiety and cognition: From impaired performance under low cognitive load to reduced anxiety under high load. Psychophysiology, 49, 842-852. doi: 10.1111/j.14698986.2012.01358.x

Wacker, J., Chavanon, M. L., Leue, A., \& Stemmler, G. (2008). Is running away right? The behavioral activation-behavioral inhibition model of anterior asymmetry. Emotion, 8, 232-249. doi: 10.1037/1528-3542.8.2.232

Wager, T. D., Phan, K. L., Liberzon, I., \& Taylor, S. F. (2003). Valence, gender, and lateralization of functional brain anatomy in emotion: A meta-analysis of findings from neuroimaging. NeuroImage, 19, 513-531. doi: 10.1016/s1053-8199(03)00078-8

Wahbeh, H., \& Oken, B. S. (2013). Peak high-frequency HRV and peak alpha frequency higher in PTSD. Applied Psychophysiology and Biofeedback, 38, 57-69. doi: $10.1007 / \mathrm{s} 10484-012-9208-\mathrm{Z}$

Weigand, A., Grimm, S., Astalosch, A., Guo, J. S., Briesemeister, B. B., Lisanby, S. H., . . . Bajbouj, M. (2013). Lateralized effects of prefrontal repetitive transcranial magnetic stimulation on emotional working memory. Experimental Brain Research, 227, 43-52. doi: $10.1007 / \mathrm{s} 00221-013-3483-7$

Weinberg, I. (2000). The prisoners of despair: Right hemisphere deficiency and suicide. Neuroscience \& Biobehavioral Reviews, 24, 799-815. doi: 10.1016/s01497634(00)00038-5 
Wiedemann, G., Pauli, P., Dengler, W., Lutzenberger, W., Birbaumer, N., \& Buchkremer, G. (1999). Frontal brain asymmetry as a biological substrate of emotions in patients with panic disorders. Archives of General Psychiatry, 56, 78-84.

Wittling, W., Block, A., Genzel, S., \& Schweiger, E. (1998). Hemisphere asymmetry in parasympathetic control of the heart. Neuropsychologia, 36, 461-468.

Yufik, T., \& Simms, L. J. (2010). A meta-analytic investigation of the structure of posttraumatic stress disorder symptoms. Journal of Abnormal Psychology, 119, 764776. doi: 10.1037/a0020981

Yun, R. J., Krystal, J. H., \& Mathalon, D. H. (2010). Working memory overload: Frontolimbic interactions and effects on subsequent working memory function. Brain Imaging and Behavior, 4, 96-108. doi: 10.1007/s11682-010-9089-9

Zenhausern, R., Notaro, J., Grosso, J., \& Schiano, P. (1981). The interaction of hemispheric preference, laterality, and sex in the perception of emotional tone and verbal content. International Journal of Neuroscience, 13, 121-126. doi: doi:10.3109/00207458109043311

Zoellner, L. A., Bedard-Gilligan, M. A., Jun, J. J., Marks, L. H., \& Garcia, N. M. (2013). The evolving construct of posttraumatic stress disorder (PTSD): DSM-5 criteria changes and legal implications. Psychological Injury and Law, 6, 277-289. doi: $10.1007 / \mathrm{s} 12207-013-9175-6$

Zoellner, L. A., Pruitt, L. D., Farach, F. J., \& Jun, J. J. (2014). Understanding heterogeneity in PTSD: fear, dysphoria, and distress. Depression and Anxiety, 31, 97-106. doi: $10.1002 /$ da. 22133

Zoladz, P. R., \& Diamond, D. M. (2013). Current status on behavioral and biological markers of PTSD: A search for clarity in a conflicting literature. Neuroscience \& Biobehavioral Reviews, 37, 860-895. doi: 10.1016/j.neubiorev.2013.03.024 



\section{Chapter 7}

\section{General Discussion}

A part of this chapter is published as:

Meyer, T., Otgaar, H., \& Smeets, T. (2015). Flashbacks, intrusions, mind-wandering -

Instances of an involuntary memory spectrum: A commentary on Takarangi, Strange, and Lindsay (2014). Consciousness and Cognition, 33, 24-29. 



\section{Summary of this dissertation}

Many people are exposed to severe negative events in their life, and there are large individual differences in how people respond to, or recover from, such events. The present work was inspired by the question why some people appear to be resilient to aversive experiences, whereas others develop and maintain pathological symptoms afterwards. An underlying idea is that it may be possible to link resilience to measurable individual characteristics - which could eventually be used to make prognoses, monitor treatment outcomes, develop novel treatment interventions, and more generally, to better understand trauma-related psychopathology. As well, understanding the individual characteristics that are associated with resilience would enable us to predict psychological adjustment even before the occurrence of a traumatic event. This knowledge could thus be used to help prevent posttraumatic stress disorder (PTSD) in individuals with a high risk of trauma exposure. With this in mind, the chapters of the current dissertation focused on various person characteristics as potential predictors of emotional adjustment, particularly following negative and stressful experiences. The characteristics that we studied can be grouped into factors relating to memory formation on the one hand (Chapters 2 and 3), and to the regulation of emotion, on the other (Chapters $4-6$ ). The more specific content of the chapters is summarized below. Next, we will highlight several key findings, issues, and conclusions.

Following a general introduction in Chapter 1, two chapters evaluated the basic idea of the dual-representation model of PTSD (Brewin, Gregory, Lipton, \& Burgess, 2010), namely that two distinct but interacting memory systems in the brain account for the occurrence of intrusive trauma memories. According to this model, the formation of allocentric, contextbased memory in the hippocampal area should reduce, and perhaps even prevent, the occurrence of intrusions after aversive experiences. Thus, individual differences in the efficiency of hippocampal-area based memory formation might be related to the risk of developing intrusions after trauma. To test this prediction, we used the Spatial Contextual Cueing Task (SCCT; Chun \& Jian, 1998) to measure individual differences in an implicit form of spatial configuration learning. In particular, the SCCT measures the degree to which spatial configurations of multiple simple cues are bound in memory, a process known to critically depend on the hippocampal area.

Chapter 2 tested whether participants' configuration learning performance on the SCCT would predict the number of intrusions over the course of one week following exposure to a trauma film. We also investigated effects on physiological responses to cues that reminded 
participants of the film. Better SCCT performance predicted fewer intrusions during a 1-week period, and especially fewer intrusions that were purely perceptual in nature. In contrast, configuration learning performance was not predictive of the distress caused by the intrusions and overall PTSD symptoms. Also, there was no association with eye-blink startle responses during or shortly after presentation of reminder pictures that served to activate memories of the trauma film. Together, these findings suggest that information processing in the hippocampal area plays a specific role in PTSD-like intrusions, but not in the emotional reaction to these unwanted memories.

Chapter 3 tested another prediction that can be derived from the dual-representation model of intrusions (Brewin et al., 2010). In particular, if the development of intrusions is linked to the efficiency of hippocampal-area based information processing, then this efficiency would be expected to be reduced under acute stress. We thus tested the hypothesis that SCCT learning performance would be impaired when participants are acutely stressed. Our findings overall did not support this hypothesis. Rather, endogenous cortisol secretion in response to stress moderated the effect of stress on SCCT learning performance. Participants with moderate cortisol reactions had reduced post-stress SCCT scores, whereas participants with high cortisol responses displayed a tendency towards amplified post-stress SCCT learning scores. This moderation effect was also supported by a positive correlation between cortisol responding and mean SCCT learning scores in the stress condition. Thus, cortisol secretion appears to protect against the adverse effects of stress on hippocampal-area based learning performance.

Next, this dissertation turned to the idea that the ability to regulate emotions is crucial in emotional well-being and in adjusting to negative experiences. In particular, Chapter 4 investigated the affective consequences of two widely studied emotion regulation strategies: cognitive reappraisal and expressive suppression. Cognitive reappraisal influences the generation of emotions by changing cognitions about the situation, whereas expressive suppression targets bodily responses and response tendencies that are activated once the emotion has been fully generated (Gross \& Thompson, 2007). It is widely assumed that reappraisal is efficient and beneficial, whereas suppression has counterproductive effects in emotion regulation. To test these assumptions, we investigated whether habitual use of these strategies would predict emotional recovery after mood induction, as well as everyday affect characteristics. Our data showed that reappraisal generally dampened affect, both negative and positive, after recovery from our mood induction. In line with this pattern, reappraisal also correlated moderately with lower diurnal arousal scores, but not with valence scores. 
Meanwhile, the data on expressive suppression yielded no clear support for the a-priori hypotheses. That is, suppression was entirely unrelated to diurnal valence or arousal metrics and had no influence on affective recovery from our emotional provocation. Suppression only predicted higher maximum fear responses. Together, our findings suggest that the beneficial effects of reappraisal come at the cost of lower arousal, and hence also lower positive affect. Meanwhile, the alleged maladaptive effects of expressive suppression may be limited in time.

The next two chapters addressed the practical and theoretical relevance of frontal asymmetry, a more objectively quantifiable marker, in relation to well-being and adjustment to stressful experiences. This biomarker is measured with electroencephalography (EEG) and is thought to reflect the relative activity of two lateralized brain systems sub-serving motivation and emotion, which would determine the individual's style of affective responding in emotional situations. Frontal asymmetry is usually measured during a resting state of several minutes. This metric is generally regarded and used as a trait-like psychological construct, labelled trait frontal asymmetry. In addition, more recent studies have measured state-induced changes in frontal asymmetry to quantify individual differences. Both resting and state-dependent asymmetries appear to be intimately associated with emotional responding and psychopathology. In this dissertation, we addressed the potential involvement of frontal asymmetry in the emotional adjustment to shocking or traumatic experiences. In particular, we investigated empirically whether frontal asymmetry would predict startle response following the activation of aversive memories. Furthermore, we reviewed empirical studies on the role of frontal asymmetry in affective processing, its possible neuropsychological origins, how it may be linked to abnormalities in PTSD, as well as what the literature says about the association between frontal asymmetry and PTSD.

More in detail, $\underline{\text { Chapter } 5}$ tested whether frontal asymmetry can predict physiological self-regulation in response to reactivation of aversive memories. In two studies, frontal asymmetry was measured both at rest and during viewing of a trauma film, and later on we assessed how the presentation of film-reminder images modulates eye-blink startle responses. In study 1 of this chapter, we explored whether the type of trauma film would moderate potential effects. In line with expectations, left-sided frontal activation during film viewing predicted dampened startle responses after the presentation of reminder pictures, but only in individuals who viewed a staged road accident. For participants who viewed a genocide documentary, this effect tended to be reversed. As a post-hoc explanation, we proposed that cognitive regulation of emotion might have been more difficult for those who saw the genocide film condition, as compared with the road accident film condition. This may have 
made emotion regulation attempts less successful. We therefore conducted a second study, where all participants viewed the genocide film. Here, we manipulated the use of reappraisal by providing positive and negative contextual information about the film. Again, we found an effect that was contrary to our initial expectation, with left frontal activity at rest predicting higher startle responses. The reappraisal manipulation did not moderate the effects. The chapter concludes by pointing towards the apparently critical role of the type of trauma film in the effects of frontal asymmetry, which warrants further critical investigation.

Chapter 6 reviewed the frontal asymmetry literature, where studies have typically found that more left-sided frontal activity is related to approach motivation and to lower levels of depression and anxiety. Based on this, one would expect frontal asymmetry to be associated with PTSD as well, because this disorder shares dysphoria- and fear-related symptoms with depression and anxiety disorders. In addition, several lines of research provide plausible links between functional asymmetries in the brain and the neuropsychological abnormalities that characterize PTSD. However, neuroimaging studies rarely perform explicit tests of laterality effects, and only eight studies directly tested the association between frontal EEG asymmetry and PTSD. These studies provide no or only very limited support that resting frontal EEG asymmetry is associated with PTSD. Meanwhile, state-induced asymmetry during traumarelevant stimulation might distinguish trauma victims with and without PTSD. The chapter concludes by highlighting hypotheses that may guide future research and help to fully evaluate the practical and theoretical relevance of this promising biomarker. Notably, the investigation of provocation-induced asymmetries appears to be promising. Finally, we point out that frontal asymmetry could be a transdiagnostic marker of psychological adjustment, and future research may want to investigate its relationship with more specific symptom clusters rather than diagnostic status.

\section{Mechanisms behind involuntary trauma memory}

By showing that better hippocampal-area based spatial configuration learning is associated with fewer perceptual intrusions of a trauma film, Chapter 2 provides support for a specific prediction of the dual representation model of intrusions (Brewin et al., 2010). In particular, our findings support the idea that the efficiency of a context-based memory system in the temporal lobe accounts for the likelihood of re-experiencing symptoms following traumatic experiences. This interpretation also aligns with prior findings of impaired spatial processing abilities in PTSD patients, and with other tests of the dual representation model 
that linked intrusions to impaired visuo-spatial processing and learning abilities (Bisby, King, Brewin, Burgess, \& Curran, 2010; Gilbertson et al., 2007). While the present work lends plausibility to the dual representation model, it also leaves certain questions unanswered and suggests new routes of research that merit to be explored. One reason is that Chapter 2 and 3 are essentially based on neuropsychological predictions. That is, we probed the efficiency of temporal-lobe based memory formation using the SCCT, based on prior neuropsychological and neuroimaging studies using this paradigm. In other words, the psychological mechanisms linking spatial configuration learning, stress, and intrusions were not of primary interest, and still remain to be understood. The following section highlights potential routes of further investigation.

For instance, in Chapter 2, we argued that the contextual cuing effect may reflect implicit processing in the parahippocampus (Preston \& Gabrieli, 2008), an important input structure for spatial representations to the hippocampus (Fyhn, Hafting, Treves, Moser, \& Moser, 2007). On a cognitive level, this could mean that the contextual cueing effect is a precursor of other (e.g., more explicit) forms of spatial learning. For instance, contextual cueing appears to rely on abstract, but still egocentric spatial representations (Chua \& Chun, 2003). These might serve as the basis for constructing view-point independent, allocentric spatial representations. People with stronger contextual cueing might thus also have better spatial memory performance on tasks involving location or trail learning and spatial navigation. Such more complex abilities might mediate the effects of implicit spatial configuration learning on intrusion symptoms. Suggestive support for this idea comes from research that mirrors our findings with the SCCT in Chapters 2 and 3; more complex spatial learning abilities have been associated with the development of intrusions (Bisby et al., 2010), and they have been shown to be susceptible to stress during the learning phase (Taverniers et al., 2011). In sum, an interesting route for future research is to investigate psychological functions that are directly associated with efficiency of visuospatial configuration learning, so as to better understand their psychological function in intrusive memory.

In order to inform clinical models of intrusions and PTSD, a related route for future research is to investigate the mechanism by which the formation of allocentric, abstract, and contextually embedded memories suppress trauma intrusions. The dual-representation model (Brewin et al., 2010) ascribes importance to the translation of sensation-near representations into context-based memory, because this prevents highly distressing sensation-based trauma memory from being activated in isolation. This implies not only that the formation of contextbased memory is important, but also that context-based representations must be accessible 
whenever a sensation-based trauma representation is reactivated. Thus, to further strengthen the evidence base of the dual-representation model, future studies should test directly how context-based memory representations change, moderate, or inhibit, the activation of sensation-near memory representations. One way to achieve this may be to rely on explicit memory paradigms, in which the visual (see, e.g., van Ast, Cornelisse, Meeter, Joëls, \& Kindt, 2013) or conceptual context (e.g., Pearson, Ross, \& Webster, 2011) of stimuli is manipulated at encoding or retrieval. This may help to elucidate the role of context in emotional memory and intrusions induced by viewing trauma films.

\section{Interactions with neuroendocrine stress responses}

Our findings in Chapter 3 indicate that exploring the role of neuroendocrine responses in trauma memory may be enlightening. That is, we found that cortisol responses moderated the impact of acute stress on the contextual cueing effect. Therefore, stress in interaction with neuroendocrine responses might be an important factor in the relation between spatial configuration learning and intrusions. Hypothetically, one individual may have an excellent ability to encode spatial configurations under normal circumstances, but display strong impairments under stress. The opposite may be the case in a second individual: relatively weak configuration learning but, due to the protective effects of cortisol secretion, no additional impairments under stress. As a result, both individuals would display the same (low) learning ability in acutely stressful situations, including traumatic events that lead to the formation of intrusions. Thus, it might be that configuration learning performance is a more reliable predictor of intrusions when it is measured during acute stress, or when individual cortisol responses to stress are additionally taken into account. Tentative support for this idea comes from our finding that cortisol responding correlates positively with mean SCCT learning scores under stress. Another indication comes from a recent study by Chou, La Marca, Steptoe, and Brewin (2014a), who found higher cortisol levels to be associated with less vivid trauma-film induced intrusions. A study that would methodologically combine Chapters 2 and 3 could help to clarify this issue.

A related issue that potentially complicates matters is timing. For instance, at what point in time, relative to a traumatic experience, do different memory processes cause or prevent intrusions? In the above-mentioned individuals, both display impaired spatial memory functioning during a traumatic event. Consequently, the peri-traumatic failure to form context-based memories may give rise to intrusive trauma memories in both individuals. 
However, later, when both return to safety and the hormonal effects of stress on memory wane, one person's spatial learning abilities would improve, whereas the other's would not. This could mean that the two might differ in how quickly the intrusions decline over time. Thus, in this model, context-based memory formation during trauma determines the development of intrusions, whereas context-based memory formation under normal circumstances determines how long intrusions persist.

A related question is at what time, and in what way, neuroendocrine stress responses modulate memory functions that give rise to trauma intrusions. As discussed in Chapter 3, a prominent idea is that basal cortisol levels and stress induced cortisol levels impair the retrieval of trauma memory, and thereby prevent intrusions (e.g., de Quervain, Aerni, Schelling, \& Roozendaal, 2009). This idea is based on studies showing that cortisol elevations during memory retrieval typically impair performance (e.g., Smeets, 2011; Smeets, Otgaar, Candel, \& Wolf, 2008) (for reviews, see Schwabe, Wolf, \& Oitzl, 2010; Wolf, 2008), and on recurrent findings of hypocortisolism in PTSD patients (Wingenfeld \& Wolf, 2011). To complicate matters, accumulating evidence suggests that the effects of neuroendocrine responses on memory are complex and time-dependent (e.g., Quaedflieg, Schwabe, Meyer, \& Smeets, 2013). Our results additionally suggest that cortisol may not only suppress memory retrieval, but might also foster adaptive information processing that is associated with spatial configuration learning. Given our findings in Chapter 2, cortisol may thus protect or facilitate memory functions that counteract intrusions. In sum, the effects of cortisol on different memory functions, and their temporal dynamics, warrant careful and systematic investigation. Intriguingly, this might lead to the development of powerful treatment options in PTSD patients (see, e.g., de Quervain et al., 2009; de Quervain \& Margraf, 2008; Soravia et al., 2006).

\section{Preventing or treating intrusive trauma memory}

A systematic investigation of the interaction of specific memory systems may lead to improved psychological interventions that target flashbacks in trauma victims. Notably, the mechanisms underlying the most successful psychotherapies of PTSD are still not fully understood. For instance, standard theories of memory would predict that reactivating and rehearsing trauma memories makes them stronger. Yet, in what seems to be a paradox, successful therapies for PTSD rely on exposure to traumatic memories or cues, and still help to reduce intrusions (Bradley, Greene, Russ, Dutra, \& Westen, 2005; Foa et al., 1999). This 
highlights that it is important to consider different memory mechanisms in order to understand the development of intrusive trauma memories, as well as routes for intervention (for discussion, see Brewin, 2014a). In terms of the dual-representation model, interventions involving rehearsal of trauma memories likely activate sensation-near trauma representations, or even strengthen them, rather than reduce or suppress them. Accordingly, the therapeutic effect is more likely to be due to enhanced formation of context-based representations, which reduces the likelihood of intrusions. Future research into these mechanisms is warranted, because it may enable the optimization of therapies. For instance, in line with the reasoning of Chapter 2, one thus could expect the efficiency to form context-based memories to predict treatment success. If this is indeed the case, it might help to develop and assign more tailored and efficient treatments.

Research along these lines may also advance the development of novel interventions. For instance, studies with trauma films have shown that carrying out specific dual tasks during or after film viewing can reduce involuntary film memories in the following weeks (Brewin, 2014a; Holmes \& Bourne, 2008; Krans, Naring, Holmes, \& Becker, 2010). The beneficial effect appears to be specific to visuospatial dual tasks (e.g., Tetris, spatial pattern tapping) and does not occur with verbal tasks. This coincides with the finding that PTSD patients, who have just experienced a flashback, display acute impairments on visuospatial, but not verbal, tasks (Hellawell \& Brewin, 2002). Thus, visuospatial tasks appear to compete with traumatic flashbacks for the same cognitive resources. It might be possible to exploit this effect to prevent intrusions in trauma victims. In order to do so, however, it is important to understand how a brief visuospatial task can lastingly prevent intrusions.

Following the dual representation model (Brewin et al., 2010), one explanation might be that visuospatial tasks disrupt perception-near trauma memories, and hence, degrade the perceptual content of intrusions. Some support for this comes from studies showing that the vividness and emotionality of memories can be reduced by activating them and simultaneously blocking visual processing through lateral eye movements (e.g., Leer, Engelhard, \& van den Hout, 2014). Other findings indicate that visuospatial stimulation after seeing emotional pictures leads to worse picture recognition (Krans, Langner, Reinecke, \& Pearson, 2013). An alternative explanation is that sensation-based memories remain intact, but a visuospatial dual task makes them transiently inaccessible. Consequently, the individual can process trauma memories only verbally and in an abstract manner, enhancing the contextbased trauma representation, which reduces intrusions. Thus, degraded perceptual memory, enhanced memory contextualization, or both, might account for the effects of visuospatial 
interference tasks on intrusions. In sum, future research should try to pinpoint the memory mechanisms underlying known treatment and prevention approaches against trauma flashbacks. This may help to identify active components and can be used to maximize the effectiveness.

\section{Affect regulation in the aftermath of emotional challenges}

The work presented in this dissertation also investigated whether and how markers of emotion regulation may be associated with adjustment to stress and trauma. Chapter 4 focused on self-reported habitual cognitive reappraisal and expressive suppression. Our data showed that reappraisal generally dampened affective experience, both negative and positive, after recovery from our mood induction. In line with this pattern, reappraisal also correlated moderately with lower diurnal arousal scores. These findings are in line with the earlier finding that reappraisal is associated with the regulation of emotional arousal (Kuppens, Oravecz, \& Tuerlinckx, 2010). However, we found no association at all with diurnal emotional valence, or with the variability and stability of affective experiences. Furthermore, because reappraisal appeared to dampen affect only in participants who were familiar with the emotional film that we used, it is unclear whether this would apply to unexpected emotional provocations. Similarly, the data on expressive suppression yielded no clear support for the view that using this strategy is harmful. In fact, it was unrelated to diurnal valence or arousal levels and had no influence on affective recovery from our emotional provocation. Suppression only predicted higher maximum fear responses, suggesting that the alleged maladaptive effects of expressive suppression are limited in time.

In the context of this dissertation, an unresolved question is how reappraisal and suppression may relate to psychological adjustment following trauma exposure and, more specifically, to PTSD symptoms. Theoretically, both cognitive reappraisal and expressive suppression can be expected to be associated with this disorder. For instance, negative appraisals of trauma memories are considered to play an essential role in maintaining symptoms (Ehlers, 2010). More use of cognitive reappraisal could be expected to dampen this effect. Moreover, since our data point towards a special relationship with reduced emotional arousal, reappraisal might have particularly beneficial effects on PTSD hyper-arousal symptoms, as well as on the intensity of reliving symptoms. Furthermore, it has been argued that expressive suppression could be related to the tendency in PTSD patients to avoid trauma-related cues, thoughts and experiences (Boden et al., 2013). In addition, avoidance 
may paradoxically increase involuntary trauma memories, implying that suppression may be associated with higher levels of PTSD re-experiencing symptoms.

A few studies have directly tested the more specific role of reappraisal and suppression in PTSD. For instance, Moore, Zoellner, and Mollenholt (2008) measured emotion regulation and PTSD symptoms in students reporting trauma history, as well as in a sample of traumaexposed individuals from the general population. Suppression was positively associated with PTSD symptoms in trauma victims from the general population, but not in students reporting trauma, while reappraisal was unrelated to PTSD symptoms. Eftekhari, Zoellner, and Vigil (2009) also measured emotion regulation and symptoms of psychopathology in undergraduates, some of whom had been exposed to traumatic experiences. Here, participants who reported high levels of habitual reappraisal along with low levels of suppression displayed the lowest levels of psychopathology, including PTSD symptoms.

The positive correlation between PTSD symptom severity and expressive suppression, as well as the negative correlation with cognitive reappraisal, was further replicated in a large sample of survivors of different types of trauma (Ehring \& Quack, 2010) and in military veterans with PTSD (Boden et al., 2013). Supporting the intimacy of this relationship, Boden et al. (2013) reported on longitudinal data in veterans with PTSD undergoing cognitive therapy. They found that increases in the use of cognitive reappraisal and less use of suppression in the course of therapy went along with reduced PTSD symptoms. In line with the idea that reappraisal might be associated with lower arousal, use of this strategy was associated with lower hyper-arousal and re-experiencing symptoms in cross-sectional analyses, although the change in the use of reappraisal over the course of cognitive therapy did not predict decreases in these specific symptom clusters. Furthermore, in line with an association between suppression and avoidance, decreased use of this strategy, indeed, predicted less avoidance (but not re-experiencing symptoms).

All in all, there is good evidence that, at least in non-student trauma victims, reappraisal and suppression are robustly associated with PTSD symptoms. Still, very little data is available about the causal relationships between these variables. Relevant to this, Ehring and Quack (2010) addressed the idea that traumatic experiences cause decreases in adaptive and increases in maladaptive emotion regulation, especially when trauma occurs frequently and early during development (e.g., before the age of 14 years). Accordingly, they expected trauma victims with chronic early trauma to report lower levels of adaptive emotion regulation than victims with single and/or late traumatic experiences. However, when controlling for group differences in PTSD symptom severity, trauma type did not influence 
levels of reappraisal or suppression. Thus, there is no clear support for the view that traumatic experiences are causally linked to the use of these emotion regulation strategies. Thus, levels of habitual use of cognitive reappraisal and expressive suppression seem to be associated with the development or maintenance of PTSD symptoms, but seem not to be influenced by trauma exposure. Clearly, more longitudinal research is needed to elucidate the specific role of these emotion regulation strategies in the time course of adjustment to trauma and in specific PTSD symptom clusters (e.g., hyper-arousal, avoidance).

The cross-sectional and field studies reviewed in the section above have advanced our understanding of how emotion regulation strategies may be involved in PTSD in an unparalleled manner. However, interpreting their findings is troubled by the fact that emotion regulation and psychopathology cannot be segregated in time. In this respect, laboratory work such as that presented in Chapter 4 has notable advantages. In this case, we could study habitual emotion regulation as a trait-like factor that precedes (recovering from) the emotional provocation. Similarly, the trauma film-paradigm provides an excellent opportunity to study whether habitual levels of emotion regulation are prospectively associated with the development of stress-related symptoms. The following section presents previously unpublished data from two trauma film studies that are relevant to this discussion.

\section{Emotion regulation in analogue trauma: An empirical interlude}

To obtain a closer look at the relationship between reappraisal and suppression with PTSD symptoms, we analysed data from two studies that used the trauma film paradigm and in which the Emotion Regulation Questionnaire (ERQ; Gross \& John, 2003) was included as a baseline measure. The main aim of these analyses was to examine associations with PTSD analogue symptoms, which adds to the PTSD studies reviewed above. Furthermore, affective reactivity to the trauma film was measured in both studies. Thus, the data also allow a closer look at the short-term consequences of reappraisal and suppression, in addition to the insights on emotional recovery that were gained in Chapter 4.

\section{Methods}

Samples from two studies were combined for the present analyses. Study 1 refers to the study that is described in Chapter 2, and which comprised 82 participants (63 women; mean age $=21.5$ years, $S D=3.4$ ). Study 2 is an unpublished EEG study that provided data pertinent to the link between emotion regulation and intrusions, and was completed by 62 participants 
(51 women; mean age $=22.1, S D=2.1$ ). This yielded a convenience sample of 144 young adults (114 women, $79 \%)$ aged 21.8 years $(S D=2.9)$. All participants fulfilled the same inclusion and exclusion criteria (as described in Chapter 2). All were exposed to the same emotional video footage in the trauma film paradigm, consisting of a compilation of brief shocking video fragments. Participants were instructed to view the film and imagine being a witness or bystander to the depicted shocking scenes, while no instruction was given regarding the experience or expression of emotion.

Affective reactivity was measured in terms of self-reported positive and negative affect, i.e., change scores on the Positive and Negative Affect Schedule (PANAS; Watson, Clark, \& Tellegen, 1988; in Study 1, for one participant these data were lost due to technical failure). In addition, participants in both studies returned to the laboratory one week after viewing the trauma film, and completed a tailored version of the self-report PTSD Symptom Scale (PSSSR; Foa, Riggs, Dancu, \& Rothbaum, 1993), measuring analogue symptoms related to the traumatic film footage.

Correlation analyses were performed between the two ERQ subscales ( $\alpha$ s $>70$ ), PANAS reactivity ( $\alpha \mathrm{S}>.78)$, and PSS-SR scores $(\alpha=.78)$. One-tailed $p$-values of 0.05 were considered statistically significant for all analyses involving expressive suppression, following the assumption that expressive suppression would be associated with worse hedonic and symptomatic outcomes. Similarly, we expected cognitive reappraisal to predict favourable outcomes and consequently tested these correlations with one-sided tests. The only exception was for positive affect reactivity, where both a negative and a positive association can be expected based on prior findings (cf. Chapter 4).

In order to assure comparability between the two studies, we tested mean differences on all variables using independent-samples $t$-tests. Presumably due to random differences between the two studies, ERQ suppression scores tended to be higher in Study 1, $t(142)=$ $1.8, p=.07$, and participants showed a larger decrease in positive affect in this study, $t=2.3$, $p=.02$, as compared with Study 2. In addition, we tested for possible interactions in the relationships between ERQ, reactivity, and PSS-SR scores using multiple regressions with Study (dummy variable) and the respective interaction term (i.e. dummy $\times z$-transformed variable) as additional predictors. None of the interaction terms reached conventional levels of significance (reappraisal: all interaction $p s>.06$; suppression: all interaction $p s>.11$ ). 


\section{Results \& Discussion}

As can be seen in Table 7.1, both expressive suppression and cognitive reappraisal had minimal (if any) effects on affective reactivity to the trauma film and on the development of PTSD analogue symptoms. A negative correlation between reappraisal and negative affect reactivity emerged, but this effect was small and practically negligible. Reappraisal had no effect on positive affect responses, or on PTSD analogue symptoms. Similarly, suppression showed only small to medium-sized associations with more negative and less positive affect in response to film viewing, and with higher re-experiencing scores one week later.

\begin{tabular}{llc}
\hline Outcome & \multicolumn{2}{c}{ Correlation with ERQ $(95 \% C I)$} \\
\cline { 2 - 3 } & Reappraisal & Suppression \\
\hline Reactivity (PANAS) & $-.14^{*}(-.27 ; .02)$ & $.22 * *(.06 ; .37)$ \\
Negative Affect & $-.01(-.17 ; .15)$ & $-.16^{*}(.00 ; .32)$ \\
Positive Affect & & \\
& & \\
PTSD Symptoms (PSS-SR) & $.01(-.15 ; .17)$ & $.19 *(.03 ; .34)$ \\
Re-experiencing & $.06(-.10 ; .22)$ & $-.09(-.25 ; .07)$ \\
Avoidance & $.06(-.10 ; .22)$ & $-.03(-.19 ; .13)$ \\
Arousal & $.06(-.10 ; .22)$ & $.02(-.14 ; .18)$ \\
Total & & \\
\hline
\end{tabular}

Table 7.1. Pearson product-moment correlations between Emotion Regulation Questionnaire (ERQ) subscales and affective responses to the trauma film $(N=144)$.

$C I=$ confidence interval $;$ PANAS $=$ Positive and Negative Affect Schedule. PSS-SR = PTSD Symptom Scale - Self-Report. $* p<.05,{ }^{*} p<.01$ (one-tailed).

Overall, these findings add to the impression in Chapter 4 that both reappraisal and suppression play a rather limited role in affective experiences in response to emotional provocation. With respect to expressive suppression, our findings confirm that suppression has mild counterproductive effects on affective experience in response to the trauma film, but this effect is very small. This aligns well with the findings in Chapter 4, where the effects of suppression were limited in time and did not outlast the recovery period of $5 \mathrm{~min}$ following 
film viewing. Similarly, there was no clear association overall with PTSD analogue symptoms.

Regarding reappraisal, the low associations with emotional reactivity scores may not be surprising. That is, participants were instructed to imagine being a witness or bystander to the shocking scenes, and in order to follow these instructions, they may have refrained from efforts to change emotional experience during film viewing. Rather, participants with high levels of habitual reappraisal may have employed this strategy in the period following film viewing. Thus, it is more surprising that reappraisal was entirely unrelated to PTSD analogue symptoms, especially in light of the recurrent finding that PTSD patients report lower levels of cognitive reappraisal (e.g., Boden et al., 2013; Ehring \& Quack, 2010). A possible explanation could be that cognitive reappraisal is, indeed, unrelated to the initial onset of PTSD symptoms. Instead, low levels of reappraisal may be a maintaining factor in the course of the disorder.

\section{Conclusions}

Collectively, the empirical work presented in this dissertation provides only slight support for the notion that cognitive reappraisal and expressive suppression play a critical role in the psychological adjustment in the aftermath of emotional challenges. Suppression had very limited effects and appeared to influence affective experiences more in the short term. Reappraisal appeared to be involved in everyday affective experiences and in the recovery from emotional provocation, but not in the onset of PTSD analogue symptoms after viewing a trauma film. Since both reappraisal and suppression have repeatedly been linked with PTSD symptoms in clinical populations (e.g., Boden et al., 2013; Ehring \& Quack, 2010), it is an appealing interpretation that habitual emotion regulation strategies are not directly responsible for the onset of PTSD symptoms following trauma exposure, but rather moderate their persistence.

There may be several alternative explanations for our failure to observe robust associations between reappraisal, suppression, and PTSD analogue symptoms. For instance, the specific type of trauma film that was used in these studies may be part of an explanation. Importantly, in the two studies presented above, the trauma film consisted of a compilation of brief shocking video fragments. This may make it difficult for participants to engage in reappraisal, i.e., to attach more benevolent meanings to the viewed material. It is thus possible that reappraisal has more beneficial effects in response to other provocations that can more 
easily be re-interpreted in a less aversive manner. We reasoned along similar lines in Chapter 4. There, post-hoc analyses suggested that reappraisal is only associated with affective recovery from a fear-inducing fragment in individuals who already knew this fragment beforehand. Furthermore, in Chapter 5, we similarly argued that cognitive reappraisal may be easier in some trauma films and more difficult in others. Thus, once again, our findings point towards the need for more systematic research into factors that may moderate the effects of reappraisal. There is good reason to believe that the specific content of trauma-films and contextual information about provocation play crucial roles that remain to be fully understood.

\section{The puzzle of frontal asymmetry in aversive memory}

Chapter 5 addressed the role of frontal asymmetry in the regulation of physiological responses to aversive memories in two studies. As reviewed in Chapter 6, a large body of evidence suggests that more left-sided frontal activity is associated with lower reactivity to emotional provocations and with lower levels of psychopathology. Thus, we had good reasons to expect that more left-sided activity would also predict enhanced physiological selfregulation in response to trauma-film induced negative memories. However, Chapter 5 revealed that the type of film used to produce negative memories plays a crucial role in this effect. Only for participants who saw staged footage of a severe road accident, more left-sided frontal activation during film viewing predicted lower startle potentiation scores.

That this effect was absent or even reversed for participants who viewed horrific scenes from a documentary about the Rwandan genocide remains puzzling. In the second study, we experimentally manipulated cognitive emotion regulation by providing additional positive background information to one of the two groups. Participants in this group, indeed, engaged more in positive reappraisal, i.e., they interpreted the film more positively in terms of personal growth (Garnefski, Kraaij, \& Spinhoven, 2002). This did not moderate the effect of frontal asymmetry. Arguably, we failed to demonstrate a moderation effect because the use of positive reappraisal remained relatively low even in the condition aiming to increase it. As we suggest in Chapter 5, future studies may want to use film materials that make it easier to manipulate positive reappraisal, possibly in addition to stronger manipulations (e.g., instructed reappraisal, cognitive challenges).

Methodological consideration aside, our null findings give rise to questions regarding the interpretation of the data. For instance, one may wonder whether positive reappraisal is 
universally beneficial and should be expected to facilitate physiological self-regulation, irrespective of the kind of emotional provocation. Indeed, there is reason to believe that this assumption is inaccurate. For instance, as pointed out by Aldao (2013), whether any particular emotion regulation strategy is adaptive or not depends on the situational context - just as any emotion can be adaptive or inadequate in different situations. To illustrate this, consider the appraisal instructions that are typically used to manipulate subjective or physiological responses in the laboratory. For example, in order to compare positive and negative appraisals of a picture depicting a spider, Foti and Hajcak (2008) instructed participants that "this poisonous snake is about to attack", or that "this snake is harmless and is in a zoo exhibit" (p.985). If an encounter with a snake would occur in real life, both of these appraisals may be adaptive or maladaptive, depending on the situation. If the snake is harmless, it would be useless to respond with extreme fear and withdrawal responses, and these reactions might even interfere with ongoing goal-directed activities. However, failing to respond in such a way can be fatal if the snake, indeed, happens to be poisonous. In light of the unexpected pattern of findings in Chapter 5, this consideration leads to the question whether positive reappraisal can be considered as an adaptive strategy per se, when one is reminded of the genocide documentary viewed by our participants in Chapter 5 .

A similar and related concern is the interpretation of startle potentiation in the context of aversive memory. Similar to prior studies relying on the eye-blink startle paradigm (e.g., Jackson et al., 2003), Chapter 5 focused particularly on the modulation of startle one second after reminder offset, whereby higher scores can be interpreted as reflecting sustained affective processing. Inversely, lower scores reflect emotional down-regulation or affective disengagement. In response to an acute emotional provocation in the laboratory with aversive pictures such as done by Jackson et al. (2003), it appears safe to equate lower potentiation scores after stimulus offset as more adaptive responding. That is, in the laboratory situation, sustained affective processing of provocative pictures has no functional value. However, in the case of traumatic memory, there are indications that enhanced startle can serve an adaptive function. For instance, Robison-Andrew et al. (2014) recently reported that combat veterans with PTSD who responded favourably to treatment tended to display both increases and decreases in trauma-potentiated startle responses over the course of therapy, a pattern suggestive of emotional processing. In contrast, treatment non-responders displayed no clear temporal pattern in startle potentiation. This could indicate that heightened memorypotentiated startle responses may sometimes reflect enhanced memory processing, which eventually leads to a reduction of PTSD symptoms. 
In sum, our findings in Chapter 5 cause us to question two implicit assumptions about emotion regulation and startle responses. One assumption was that positive reappraisal is an adaptive cognitive response per se. After all, this may not be the case when viewing for example, a genocide documentary. As a second assumption, we conceptualized sustained memory-potentiated startle responding as a maladaptive response that is similar to PTSD hyper-arousal symptoms. However, it could be that in this condition, sustained emotional engagement reflects normal and even adaptive processing of emotional material. The brief empirical section that follows aims to elucidate these issues.

\section{Frontal asymmetry: Adding pieces to the puzzle}

With the above-mentioned considerations in mind, this section aims to shed more light on two questions. First, is positive reappraisal, indeed, an adequate and helpful emotion regulation strategy in response to the shocking documentary about crimes against humanity that we showed to our participants in Study 2 of Chapter 5? Second, does our data support the view that in these participants, potentiated startle responses following the offset of reminder pictures reflects maladaptive emotional processing?

While more studies may be necessary to answer these questions systematically, questionnaire data that was additionally collected in this study can provide relevant insights. In particular, participants completed the revised 21 item version of the Depression, Anxiety and Stress Scales (DASS-21; Lovibond \& Lovibond, 1995), which measures common symptoms of psychopathology. Its total score is an indicator of general psychological distress - a concept reflecting the tendency to experience negative affect and subjective stress, ineffective coping and enhanced risk to develop various forms of psychopathology, summarized under the broad personality factor neuroticism (McCrae \& John, 1992). Thus, this scale can serve as a separate criterion to test whether the processes of interest can be considered (mal-)adaptive processes. Positive reappraisal is typically negatively associated with neuroticism (Garnefski et al., 2002), while startle potentiation would be expected to show a positive correlation with neuroticism.

\section{Methods}

For procedural details, see Chapter 5, Study 2. The DASS-21 was administered within the questionnaire battery at the beginning of the study. Its 21 items describe psychological or somatic symptoms, and respondents indicate how frequently each of them occurred in the past 
week on a 4-point scale $(0=$ Did not apply to me at all, $3=$ Applied to me very much, or most of the time). The DASS-21 total score $(\alpha=.85)$ is calculated as the sum of all items multiplied by 2 , making it comparable to the longer 42 -item version (Lovibond \& Lovibond, 1995). The sample had an average DASS-21 total score $14.8(S D=10.4$; range: $0-60)$, indicating that all participants fell within the normative range for non-clinical adults from the general population (Henry \& Crawford, 2005). Due to their typical left-skewed distribution, DASS-21 scores were log-transformed prior to the following analyses.

Also as part of the baseline questionnaire battery, the original Cognitive Emotion Regulation Questionnaire (CERQ; Garnefski et al., 2002) was administered. The CERQ consists of nine 4-item subscales measuring the habitual use of different cognitive emotion regulation strategies that are applicable in negative events or situations. Each item requires respondents to indicate the frequency of a specific thought on a 5-point scale $(1=$ [almost $]$ never, $5=$ [almost $]$ always $)$. For the current analyses, only the positive reappraisal subscale $(\alpha$ $=.81$ ) is of interest (example item: "I think that I can learn something from the situation"). It served to explore the convergent validity of the state positive reappraisal subscale $(\alpha=.69)$ from the adapted state CERQ that was administered at the end of the study. Notably, habitual positive reappraisal is not necessarily a good predictor of state positive reappraisal during our study. Another reason why these two questionnaires may not fully converge is that in our adapted state version, the items asked respondents to indicate intensity for each item $(1=$ very slightly or not at all, $5=$ extremely) rather than frequency.

In the following, one-tailed tests of statistical significance were used for a-priori hypotheses and two-tailed tests for exploratory analyses, with alpha set at 0.05 . Prior to that, where applicable, we explored possible interactions with the two debriefing conditions (negative, positive) by entering the condition main effect (dummy variable) and the interaction term (dummy $\times z$-transformed variable) in linear regression models along with the respective predictor. Since no statistically significant interactions were found (all $p s>.10$ ), these tests are not further elaborated on below.

\section{Results and discussion}

As expected, state positive reappraisal measured at the end of the study showed a positive, but relatively small, correlation with habitual positive reappraisal, $r(72)=.25(95 \%$ $C I=.02 ; .46), p=.02$. Notably, this correlation may be weakened due to scoring and reliability differences between the original CERQ and the adapted state scale. Correlations 
between DASS-21, reminder startle potentiation, and state positive reappraisal are summarized in Table 7.2. As can be seen, no statistical associations emerged between startle potentiation scores and DASS-21 scores. Moreover, while positive correlations were expected, the correlation coefficients were negative. Together, the data clearly contradict the idea that higher startle potentiation scores would be associated with individual differences in general psychological distress, or neuroticism. Furthermore, and even more surprisingly, positive reappraisal scores were positively associated with DASS-21 scores.

\begin{tabular}{ccc}
\hline Outcome & $N$ & Correlation with DASS-21 $(95 \% \mathrm{CI})$ \\
\hline Startle Potentiation & & $-.04(-.27 ; .20)$ \\
$2.5 \mathrm{~s}$ & 70 & $-.16(-.38 ; .08)$ \\
$4.5 \mathrm{~s}$ & 70 & $-.18(-.40 ; .06)$ \\
$7.0 \mathrm{~s}$ & 70 & $.25^{*}(.02 ; .46)$ \\
State Emotion Regulation & & \\
Positive Reappraisal & 72 &
\end{tabular}

Table 7.2. Pearson product-moment correlations between Depression Anxiety and Stress Scales (DASS-21) total scores, startle responses, and state positive reappraisal. $C I=$ confidence interval; $* p<.05$ (two-tailed; effect is opposite to prediction).

To facilitate the discussion of which role frontal asymmetry may play in startle responding and emotion regulation, we also explored whether resting or activation frontal asymmetry scores would be associated with habitual or state positive reappraisal correlation analyses. Asymmetry scores were unrelated to habitual positive reappraisal, and to state reappraisal at the end of the study, all $p \mathrm{~s}>.18$. Thus, it may well be that frontal asymmetry interacts with emotion regulation, but cannot be regarded as a direct neural correlate of habitual or state positive reappraisal. Furthermore, the expected negative correlation between resting frontal asymmetry and DASS-21 scores failed to reach statistical significance, $r(71)=$ $-.15(95 \% C I=-.37 ; .09), p=.11$. Activation asymmetry also was unrelated to DASS-21 scores, $p=.48$. A possible reason for these null-findings is that our sample was relatively high-functioning (i.e., mostly undergraduate students), so that the range of both psychological symptoms and frontal asymmetry scores may have been too restricted to replicate the negative association that has frequently been found in clinical populations. 


\section{Conclusions}

Together, these data indicate that a few common assumptions about reappraisal, startle responding, and frontal asymmetry may require reconsideration. For instance, in line with what others have argued (e.g., Aldao, 2013), it appears that (positive) reappraisal should not be regarded as an emotion regulation strategy that is universally efficient or adaptive. That is, the positive association between reappraisal and general psychological distress even suggests that applying this strategy to the genocide documentary is maladaptive. In other words, thoughts like "I think that I can learn something from the situation" may even be a sign of poor emotional adjustment in this particular context. This observation adds to the observations regarding reappraisal in Chapter 4; it appears that in the immediate aftermath of emotional situations, individuals with high levels of reappraisal do not automatically fare better than those with low levels.

A similar, but inversed argument can be made for memory-potentiated startle responses. Sustained emotional processing, as indexed by potentiated startle after reminder picture offset, was not related to more general psychological distress. In fact, the correlation coefficients turned out to be negative, even if not significant. Thus, it may be inappropriate to equate sustained physiological reactivity and affective processing with unsuccessful adjustment. At this point, one may speculate that the functional significance of memory-potentiated startle differs between the two types of trauma film that we used in Chapter 5. That is, for individuals who had seen the staged road accident, lower startle may be indicative of affective disengagement as part of an adaptive response. In contrast, in response to the genocide film, such affective disengagement may be part of an avoidant response that prevents adequate cognitive and affective processing of the experience, thereby maintaining negative affect. Unfortunately, we did not measures the degree of positive reappraisal or levels of general psychological distress in Study 1 of Chapter 5, leaving it to future research to directly test these ideas in studies employing different types of trauma film.

\section{Disentangling the role of frontal asymmetry and memory-potentiated startle}

In Chapter 5, we reasoned that frontal asymmetry may play different roles in the adjustment to different types of aversive situations. However, as our line of reasoning above shows, one may as well think that frontal asymmetry exerts the same influence across different types of traumatic situations. Accordingly, in our experiments, where participants viewed either a staged road accident or a genocide documentary, the functional meaning of 
startle potentiation differs, just as the functionality of applying cognitive reappraisal to the situation. Supporting this view, our data show that frontal asymmetry is not directly related to reappraisal. In that case, what precisely is the role of frontal asymmetry in physiological responses to aversive memories?

According to the motivational direction model of frontal asymmetry (Davidson, 1998), more left-sided activity is linked to more approach and less withdrawal/avoidance motivation. Speculatively, when confronted with an emotional challenge, higher approach motivation might translate into more active cognitive and emotional processing of the provocative material, whereas higher withdrawal motivation might translate into avoidance. Importantly, information processing accounts of fear and anxiety (e.g., Foa \& Kozak, 1986) attribute great importance to emotional processing as a normal reaction following traumatic experiences, because it leads to the reduction of negative affect, to restoring a sense of safety, and to adjusting beliefs about the self and the world (also see Brewin, Dalgleish, \& Joseph, 1996). Enhanced emotional processing is also one of the mechanisms believed to underlie the therapeutic effects of exposure therapy (for a review, see Tryon, 2005). Accordingly, higher left-sided frontal activity could be expected to promote adjustment, irrespective of the specific content of the emotional situation.

Following this line of reasoning, the association between frontal asymmetry and startle potentiation can still differ between different types of emotional provocation, because the time course of emotional recovery will differ, depending on the severity of the provocation. As recent findings in PTSD patients suggest (Robison-Andrew et al., 2014), successful recovery from trauma and PTSD symptoms may be preceded by heightened trauma-potentiated startle reactivity. Arguably, such an increase reflects enhanced emotional processing, which might be associated with higher left-sided frontal activity (cf. supra). The duration and intensity of this emotional processing phase likely depends on the severity of the emotional provocation. Therefore, participants in Chapter 5 who watched the staged road accident may have been able to reduce negative affect much more quickly than those who saw the genocide documentary. Indeed, the subjective reports of negative emotions are in line with this view. As a consequence, the startle paradigm that was administered directly following exposure to the trauma film may have measured the emotional processing phase in the genocide condition, whereas in the road accident condition, most participants had already fully recovered from the provocation.

These considerations illustrate that our two studies in Chapter 5 have uncovered an intriguing pattern of physiological responding to aversive memories that may have important 
implications for our understanding of psychological adjustment to trauma. At the same time, these findings also make it clear that we currently have an incomplete picture of the affective dynamics that are set off by exposure to the two very different emotional provocations that we used. That is, it is plausible that the studies presented here only give us a glimpse at the dynamic interplay between frontal asymmetry, memory-potentiated startle, and psychological adjustment. Accordingly, an interesting route for future research would be to adopt a more dynamic view of adjusting to trauma, taking the time course of emotional responding into account. For instance, as in Chapter 2, it would be informative to follow up participants for the assessment of PTSD analogue symptoms. Moreover, it appears worthwhile to explore potential psychological processes that mediate the relationships between frontal asymmetry, cognitive reappraisal, and startle, with successful adjustment, such as active versus avoidant emotional processing.

More research is also needed before the findings in Chapter 5 can form a coherent picture with the literature on frontal asymmetry in PTSD that we reviewed in Chapter 6. There, we found only little support for the idea that resting frontal asymmetry is directly associated with PTSD symptoms, whereas initial evidence suggests that asymmetry measured during trauma cue exposure might be diagnostic of PTSD (e.g., Rabe, Beauducel, Zöllner, Maercker, \& Karl, 2006; Rabe, Zöllner, Beauducel, Maercker, \& Karl, 2008). In contrast, our findings in Chapter 5 indicate that both resting and provocation-induced asymmetries are related to physiological self-regulation in response to aversive memories, but in a complex and context-dependent manner. Thus, there appear to be explanatory gaps in the literature that should be closed from two sides. First, as mentioned above, future experimental studies into (laboratory-induced) aversive memory should extend their focus on the role of frontal asymmetry and emotion regulation in the temporal dynamics of negative affect, on longer timescales. Second, studies in clinical populations should focus on more precisely delineated symptom clusters rather than overall PTSD diagnosis or severity (see for a similar argument, Galatzer-Levy \& Bryant, 2013). As discussed in Chapter 6, there is no reason to believe that all symptoms that belong to the PTSD diagnosis are equally well linked to frontal asymmetry. Finally, both analogue-trauma and clinical studies with PTSD patients on frontal asymmetry may benefit from combining neuroimaging methods to achieve a more fine-grained functional understanding of asymmetric brain processes. 


\section{Bringing together analogue and clinical studies on PTSD}

One of the aims of the empirical work presented in this dissertation was to provide explanations for the occurrence of intrusive memories and other PTSD symptoms. Notably, we made use of analogue designs in healthy participant samples, including the trauma film paradigm (Holmes \& Bourne, 2008). This type of study has a number of advantages over studies relying on traumatized samples, because the analogue trauma occurs under laboratorycontrolled conditions. This allows control over the type and intensity of the aversive experience, which is often a problem in studies that include trauma-exposed individuals from the general population. In addition, the use of a relatively homogenous and high-functioning sample of participants reduces the influence of (pre-trauma) psychopathology that may overshadow the effects under investigation. However, one should keep in mind that the methodological approach used in these studies limits the generalizability of the findings.

Importantly, healthy participants typically experience only very mild symptoms that differ dramatically from more severe symptoms of PTSD patients. Moreover, the possibility cannot be excluded that PTSD symptoms differ not only quantitatively, but also qualitatively from involuntary memories assessed in analogue studies (Brewin, 2014a; Kvavilashvili, 2014). This limitation needs to be kept in mind when interpreting the empirical findings of this dissertation. At the same time, careful consideration of differences and similarities between analogue and clinical studies can also be highly insightful and contribute to a better understanding of mental health and psychopathology. The following section presents a commentary on a recent research paper authored by Takarangi, Strange, and Lindsay (2014). The commentary elaborates on the issue of bringing together analogue and clinical studies on trauma memory. Thereby, this commentary exemplifies potential problems and opportunities of bringing together analogue and clinical studies on PTSD.

In their paper, Takarangi et al. (2014) showed in two experiments that participants who had witnessed a shocking film frequently "mind-wandered without awareness" about the content of the film. More importantly, they equated this effect with the occurrence of traumatic intrusions. In this commentary, we argue that the authors have conceptually misused terms with the possible consequence of creating a conceptual blur in the traumamemory field. We postulate that clear definitions are urgently needed for phenomena such as intrusions, flashbacks, and mind-wandering, when using them in the context of trauma memory. Furthermore, our proposal is that these phenomena can fall under a spectrum of different involuntary memory instances. We propose that by adopting stricter definitions and 
viewing them as separate, but interrelated phenomena, different lines of trauma-memory research can be reconciled, which would considerably advance the field.

Flashbacks, intrusions, mind-wandering - Instances of an involuntary memory spectrum: A commentary on Takarangi, Strange, and Lindsay (2014)

Takarangi et al. (2014) examined whether individuals who had just witnessed a shocking film would think about this film during an unrelated reading task without being aware of it. For this purpose, participants in two separate experiments were instructed to press a button each time they caught themselves thinking about the trauma film while performing the reading task. The authors referred to these self-reports as "mind-wandering with awareness". In each study, a subset of participants were additionally asked, at unpredictable times and independently of the self-caught mind wandering, whether they were currently thinking about the trauma film. When participants affirmed these probes, the researchers counted this as "mind-wandering without awareness". In the two experiments, the authors found that participants "mind-wandered without awareness" on average on $29 \%$ and $40 \%$ of the probes.

Mind-wandering without awareness also had cognitive side effects, in that it correlated with deteriorated performance on the reading task. This correlation did not emerge for selfcaught mind wandering. The authors subsequently discussed that their findings bring together two lines of research; one using paradigms that probe meta-awareness during mind-wandering (for review, see Schooler, 2002; Smallwood \& Schooler, 2015), and another one using the trauma-film paradigm (Holmes \& Bourne, 2008) to study involuntary negative memories that serve as a laboratory analogue for trauma memories in post-traumatic stress disorder (PTSD). This way, their article suggests new and interesting routes of research on uncharted territory.

In this commentary, we argue that while Takarangi et al. (2014) describe a promising new line of research, the authors have used certain terminology in a rather liberal, and perhaps even conceptually ambiguous, way. Essentially, in our opinion, mind-wandering without awareness is distinct from other forms of involuntary memory. More importantly, we believe that not distinguishing between mind-wandering and other types of involuntary memory has the potential to unlock a conceptual fuzziness in the trauma-memory literature. Below, we elaborate on our main objection to the way the authors presented their findings. Then, we sketch in what way phenomena such as mind-wandering, intrusions, and flashbacks should be viewed in order to avoid confusion and unnecessary controversy. Finally, we briefly outline implications for future research and indicate how clearer definitions may advance the field. 


\section{The giant leap from mind wandering to trauma intrusions}

Takarangi et al. (2014) claimed to investigate whether "people would sometimes fail to recognize the occurrence of traumatic intrusions" (p.298). Based on the observation that participants were sometimes caught mind-wandering without reporting it, the authors came to the conclusion that "self-report may underestimate intrusions" (title). We argue that equating mind wandering with traumatic intrusions is a conceptually big leap, which carries with it the risk of exacerbating an already existing confusion and conceptual blur around the term intrusion. In our view, mind-wandering without awareness should not be equated with other forms of involuntary trauma memory, such as intrusions. Rather, we propose to interpret it as a separate phenomenon that can be distinguished from, but also shares features with, fairly common involuntary autobiographical memories. Furthermore, we show that it may nonetheless be remotely related to more severe intrusive flashbacks that are experienced by trauma victims who suffer from PTSD.

The idea that mind-wandering in the absence of awareness can be equated to traumatic intrusions likely traces back to the recent work of Baird, Smallwood, Fishman, Mrazek, and Schooler (2013). These authors used a similar probe-catching method as Takarangi and colleagues and showed that participants frequently mind-wander towards emotional memories without being aware of it, even though they are explicitly trying to suppress these memories. Baird and colleagues gave this phenomenon the provocative name "unnoticed intrusions". From the perspective of consciousness research, this term makes perfect sense because it reflects the idea that unwanted thoughts intrude into experiential consciousness in the absence of meta-awareness. However, this wording is problematic in the context of trauma, because it gives the false impression of alluding to intrusive trauma memory, a term that is often used interchangeably with involuntary autobiographical memory, or intrusive flashback. The field has yet to agree on clear definitions and distinctions between these concepts (Brewin, 2014b; Kvavilashvili, 2014). Using the term mind-wandering as another substitute or interchangeable term for intrusive trauma memory incites the impression that the processes underlying these phenomena are identical.

However, it is clear that mind-wandering without awareness does not qualify as any of these phenomena. Intrusions elicited with the trauma-film paradigm (Holmes \& Bourne, 2008) are a self-reported phenomenon that is defined by introspection. Typically, participants are instructed to record sudden, unwanted memories of the film footage (e.g., when images from the film suddenly pop into mind). Notably, the analogy of mind-wandering suggests a 
smooth transition rather than a sudden popping into mind. In line with this, non-conscious cognitive processes are thought to be continuous, whereas meta-awareness is considered to occur intermittently (Schooler, 2002). Besides, although non-conscious mental processes may be considered involuntary, the person experiencing them cannot appraise them as unwanted, let alone intrusive, unless he or she becomes aware of them. Thus, trauma-film intrusions require meta-awareness.

A similar case can be made for some of the re-experiencing symptoms in PTSD. According to the DSM-V (American Psychiatric Association, 2013), these comprise "spontaneous or cued recurrent, involuntary, and intrusive distressing memories of the traumatic event(s)" that are similar to trauma-film intrusions in terms of meta-awareness. In addition, the DSM-V lists "dissociative reactions (e.g., flashbacks) in which the individual feels or acts as if the traumatic event(s) were recurring. (Such reactions may occur on a continuum, with the most extreme expression being a complete loss of awareness of present surroundings)". According to this definition, meta-awareness can be present or absent, depending on the severity of the symptoms. Nonetheless, the presence of all re-experiencing symptoms is established by questioning (e.g., using structured interviews or questionnaires), which requires at least retrospective meta-awareness. Therefore, it is clear that PTSD reexperiencing symptoms, as defined in research and clinical practice, do not include phenomena akin to unnoticed intrusions.

\section{The spectrum of involuntary trauma memories}

Despite the above-mentioned critique that mind-wandering should not be fully equated with intrusions and traumatic flashbacks, we agree with Takarangi et al. (2014) that these phenomena are spontaneous thought processes that may be closely related to each other. Studying similarities and differences between these phenomena can yield important insights in trauma memory, but in our opinion, this requires the a priori setting of clear definitions. Indeed, Kvavilashvili (2014) recently argued along similar lines. She proposed that various forms of involuntary trauma memory can be presented on a continuum. Accordingly, involuntary autobiographical memories are a frequent part of everyday experiences that present almost no disruption to ongoing activities. Flashbacks in PTSD patients are situated on the opposite end of the continuum - these are highly distressing and can severely disrupt ongoing activities. Intrusive memories are situated in between these two phenomena. Importantly, all three share some features, and may be distinguished by quantitative and/or 
qualitative differences. We propose to extend this continuum by adding mind-wandering without meta-awareness as a fourth category on the lower end of the spectrum. Let us explain why.

Figure 1 displays the proposed spectrum and summarizes key variables that distinguish the four phenomena. Using the words of Takarangi et al. (2014), mind-wandering can be thought of as "fleeting involuntary autobiographical recollections", which individuals experience frequently "without becoming meta-aware that they are doing so" (p.303). The factor that most ostensibly distinguishes mind-wandering from involuntary memory is metaawareness. Furthermore, if mind-wandering is a fleeting mental process, a related qualitative difference with involuntary memories is that these latter occur suddenly. Otherwise, mindwandering and involuntary autobiographical memories share several features. For instance, they can involve any emotional valence (negative, positive, neutral), are typically low in distress and cause only very little disruption of ongoing activities. Importantly, similar to voluntary remembering, both may be integral parts of normal memory processing in healthy individuals and occur frequently in everyday life (Berntsen, 2010).

The other three phenomena can be distinguished as proposed by Kvavilashvili (2014). Figure 1 additionally highlights potential qualitative and quantitative differences. Involuntary autobiographical memories are similar to intrusive memories, but a key difference is that the latter are perceived as disturbing, unwanted (i.e., intrusive), and hard to control. Thus, a negative appraisal about having the memory and the wish to avoid it may differentiate an involuntary autobiographical memory from an intrusive memory. Finally, flashbacks can be distinguished from intrusive memories in that the individual has a sense of re-living the traumatic event rather than just remembering it. Thus, flashbacks comprise extreme dissociative reactions characterized by an overwhelming sense of re-living and "nowness". 


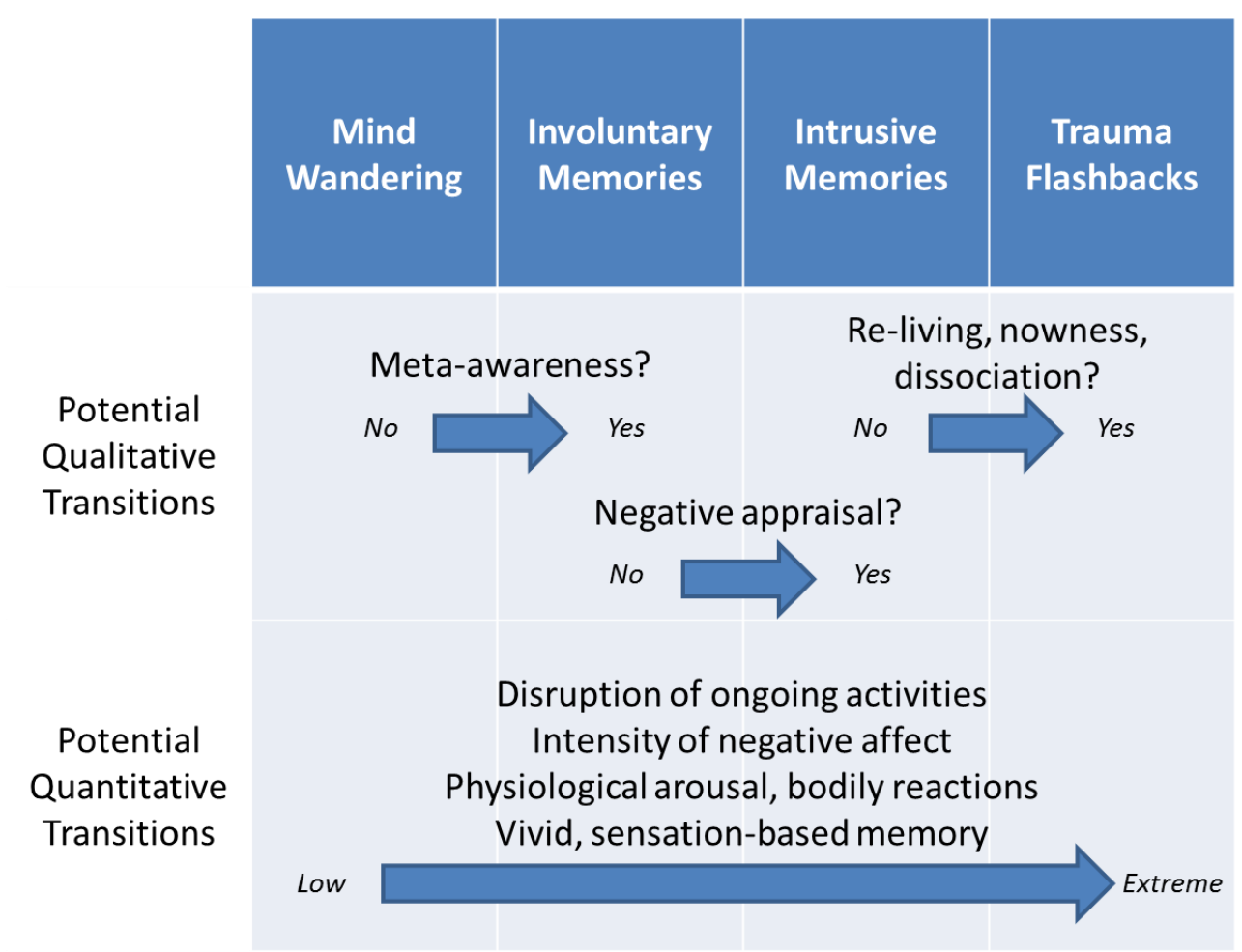

Figure 7.1. Schematic representation of different types of traumarelated memory phenomena on a continuum, whereby potential qualitative and quantitative differences between categories are highlighted.

In addition to the potential qualitative differences between the four phenomena, there also appear to be quantitative characteristics that are shared by all phenomena to different levels. One such characteristic is the degree to which the experience disrupts ongoing activities. While mind-wandering merely taps attentional resources and reduces performance on other tasks (Baird et al., 2013; Delaney, Sahakyan, Kelley, \& Zimmerman, 2010; Takarangi et al., 2014), a flashback can be so disabling that pursuing another activity in parallel becomes impossible. Similarly, the intensity of negative affect is low in mindwandering, but high to extreme in a flashback. This is also reflected in the observation that mind-wandering and involuntary memories can also relate to neutral and positive memories, while this is rarely the case for intrusive memories and even impossible for dissociative flashbacks. Closely associated with the negative emotional reactivity, similar relationships can be expected for physiological arousal and bodily reactions, including startle reactions (Brewin, 2014a; Kvavilashvili, 2014).

Finally, the four phenomena may depend to differing degrees on abstract and verbal, versus more sensation-near and perceptual, representations of the traumatic experience. 
Research has shown that these aspects of trauma memory are dissociable phenomena (Hagenaars, Brewin, van Minnen, Holmes, \& Hoogduin, 2010), and emotion specifically enhances perceptual memory (Arntz, de Groot, \& Kindt, 2005; Holmes \& Mathews, 2010). In line with this idea, mind-wandering, as studied by Takarangi et al. (2014), predominantly refers to verbal representations of the film content (they asked participants: "Just now were you thinking about the film?”; p.299). In contrast, flashbacks have been argued to be entirely based on long-term perceptual memory (Brewin, 2014a, 2014b). Accordingly, it is conceivable that involuntary autobiographical and intrusive memories lie in the middle of this continuum.

\section{Implications and conclusion}

We propose that dissociative flashbacks, intrusive memories, involuntary autobiographical memories, and now also mind-wandering, can all be regarded as instances on a spectrum of involuntary trauma memory phenomena. However, a clear definition is necessary for each of these concepts in order to avoid confusion and unnecessary controversy. We here present them on a spectrum that is based on the prior work of Kvavilashvili (2014), extended with the suggestion by Takarangi et al. (2014) that mind-wandering without awareness is closely related to traumatic intrusions. This may be helpful to refine theoretical accounts of involuntary trauma memory (Brewin, 2014a; Brewin et al., 2010; Ehlers \& Clark, 2000; Rubin, Berntsen, \& Bohni, 2008). Researchers are invited to refer more explicitly to the specific aspect of trauma memory that they aim to investigate, and to consider the qualitative and quantitative properties that would characterize it.

One might object that liberal definitions of phenomena such as flashbacks or traumatic intrusions are harmless, because they are so highly interrelated that one phenomenon implies the other. However, this strategy can be perilous. For instance, clinicians and researchers who are unaware of the intricacies of involuntary memory may mistake ordinary forms of memory processing for signs of PTSD-like reliving symptoms, only because the memory content is related to trauma (see Berntsen, 2010). This may lead to erroneous clinical decisions - and research into interventions like psychological debriefing have shown that unsolicited treatment can be detrimental to clinical outcomes (McNally, Bryant, \& Ehlers, 2003; Rose, Bisson, \& Wessely, 2003), purportedly because it may interfere with natural recovery.

Moreover, equivocal definitions account for unnecessary confusion and controversy among trauma-memory researchers (Kvavilashvili, 2014), and similar debates in other areas 
of memory research teach the same lesson. For instance, in research on false memories (i.e., memories of non-experienced events), scholars have often used the terms false belief and false memory interchangeably, implying that they are exactly the same. However, contemporary research is making it increasingly clear that believing and remembering can and should be dissociated from each other (Otgaar, Scoboria, \& Smeets, 2013; Scoboria, Mazzoni, Kirsch, \& Relyea, 2004; Smeets, Merckelbach, Horselenberg, \& Jelicic, 2005). Thus, imprecise definitions may have impeded progress in studying these separate, but related processes.

As pointed out by others (Brewin, 2014b; Kvavilashvili, 2014), extreme forms of trauma memory, such as dissociative flashbacks, may never occur in healthy individuals and cannot be studied in analogue studies using the trauma film paradigm. Thus, studies including PTSD patients are necessary. However, explicit referral to the qualitative and quantitative characteristics of involuntary memories will help to reconcile findings from analogue and clinical studies. Furthermore, as Figure 7.1 illustrates, mind-wandering, involuntary memories, intrusive memories, and trauma flashbacks can be regarded as separate phenomena, and distinguishing them would put findings from clinical, analogue, and basic memory research into perspective, making it easier to relate them to each other. Hopefully, this will stimulate methodological and conceptual convergence between these types of study, and in the end result in a better understanding of healthy and pathological responses to traumatic experiences.

For clinical models of PTSD, the inclusion of mind-wandering as a form of trauma memory leads to the question of which phenomena are clinically relevant. That is, are these mild forms of involuntary trauma memory associated with, or causally linked to the same underlying mechanism(s) as pathological flashbacks and other clinical outcomes? If so, this would plead for the validity of analogue studies, e.g., to explore the effects of treatment interventions (e.g., Holmes, James, Kilford, \& Deeprose, 2010). Moreover, milder forms of involuntary trauma memory in PTSD patients might be interesting novel targets for treatment. In contrast, if mind-wandering and everyday involuntary autobiographical memories are unrelated to clinical outcomes in PTSD, this would suggest that qualitative rather than quantitative characteristics of involuntary memory are responsible for healthy or pathological adjustment to trauma. This would indicate a crucial role, e.g., for meta-awareness, negative appraisals, or dissociation, in PTSD. More systematic research into these important questions is pressingly needed. 
In order to enable systematic research into mind-wandering, intrusions, and flashbacks, careful methodological considerations are needed. Unfortunately, still little is known about the accuracy and reliability of the currently available instruments to identify intrusions or flashbacks (e.g., structured clinical interviews). Controlled symptom provocation tests, such as script-driven imagery (e.g., Hopper, Frewen, van der Kolk, \& Lanius, 2007), trauma cue exposure (e.g., Bremner et al., 1999), or writing and reading trauma narratives (e.g., Hellawell \& Brewin, 2002) appear especially valuable for this area of study. Importantly, as pointed out by Brewin (2014b), it is crucial to determine whether the elicited memories qualify as involuntary or intrusive memories, flashbacks, or voluntarily retrieved memories. Recent adaptations of the trauma film paradigm show that these tasks can easily be implemented in analogue designs as well. For instance, based on participants' description of involuntary trauma film memories, Chou, La Marca, Steptoe, and Brewin (2014b) selected scenes that elicited intrusive memories and used them in a separate memory task. Likewise, specific trauma-film related memories can be elicited with neutral picture cues (Meyer et al., 2014; Meyer et al., 2013).

In order to also assess involuntary trauma memory that occurs outside awareness, the repertoire could be extended with a reading task that probes mind-wandering (Schooler, 2002), as Takarangi et al. (2014) have demonstrated. However, to understand the role of these processes in the build-up of intrusions and flashbacks, it would be particularly helpful to study their role in daily life. For this purpose, the diary method that is typically used in the trauma film paradigm could be adapted for both analogue and clinical studies. In particular, we agree with the suggestion of Takarangi et al. (2014) that the assessment should not only focus on the self-reported occurrence of intrusions, but should be extended with a signal-contingent experience sampling protocol (i.e., prompting with a timer). Prior research has shown that this method is able to sample mind-wandering along with characteristics of affective experience (e.g., Killingsworth \& Gilbert, 2010), and can be done even with a very high temporal resolution, allowing a closer look at temporal dynamics among multiple processes (e.g., see Kuppens et al., 2010). Similar applications in the field of trauma memory might thus lead to a better understanding of the within-person dynamics leading up to the build-up of flashbacks, as well as of between-subject moderators that determine mental health outcomes.

In conclusion, Figure 1 shows several assumptions and predictions that can inspire novel hypotheses and guide future research in the trauma memory field. As argued above, we suggest that all four instances of involuntary trauma memory share several characteristics, albeit to different degrees. This implies that they may be the products of the same underlying 
memory mechanism(s). At the same time, we postulate that qualitative differences between the phenomena exist, implying that fundamentally different mechanisms may be responsible for the occurrence of mild versus extreme forms of involuntary trauma memory.

To advance our understanding of these matters, we propose two research lines that can push the trauma-memory field a step further. First, research could establish what role mindwandering plays in PTSD, making use of studies in both traumatized and analogue trauma samples (for a helpful framework that may guide such studies, see Ehring, Kleim, \& Ehlers, 2011). Jointly, the aim should be to pinpoint the specific way in which trauma-related mind wandering is related to other forms of involuntary trauma memory (e.g., onset and/or maintenance of flashbacks). To test a causal role in PTSD re-experiencing symptoms, it may be possible to manipulate trauma-related mind-wandering experimentally following exposure to a trauma film, for instance by increasing participants' moment-to-moment awareness in a mindfulness-based intervention (e.g., Geschwind, Peeters, Drukker, van Os, \& Wichers, 2011). If such an intervention can be shown to reduce flashbacks, this might pave the way for the development of novel treatment interventions for PTSD. A second line of research could aim to explain why one individual may merely mind-wander about a recent trauma, whereas another person may suffer from highly distressing intrusions and flashbacks following a similar experience. Again, this will require both clinical and experimental laboratory work and a careful extension of the existing methodology to assess a larger spectrum of traumarelated cognition. In addition, future research should thus explicitly look for those cognitive factors that cause mind-wandering to become an involuntary memory, or an intrusive memory to become a flashback (e.g., meta-awareness, appraisals processes, dissociation).

\section{The adaptive functions of memory formation, emotion regulation, and frontal brain asymmetry in PTSD}

Above, we have argued that clinical and experimental studies can complement each other and can jointly advance our understanding of psychological adjustment following stress and trauma. This also applies to understanding how memory formation, emotion regulation, and frontal EEG asymmetry are involved in PTSD. In this context, it is worthwhile to consider the research framework by Ehring et al. (2011). These authors pointed out that studies with traumatised individuals and analogue studies are required to fully evaluate the involvement of specific cognitive factors in PTSD. Ehring and colleagues suggest that, at first, statistical associations between a candidate factor and PTSD symptoms should be established in both clinical and analogue studies. A next step would be to evaluate the 
principle of causality by experimentally manipulating the factors under investigation. If successful, the gained knowledge can be used to test clinical implications and develop novel interventions to treat or prevent PTSD. To exemplify their line of reasoning, Ehring et al. demonstrated how various studies have helped to establish perceptual priming for trauma cues and trauma-related rumination as mechanisms involved in the development and maintenance of PTSD symptoms, respectively.

Based on the work and literature presented in this dissertation, similarly specific roles in PTSD seem to emerge for at least some of the factors that were investigated here. For instance, hippocampal-area based spatial configuration learning and processing abilities appear to be associated with intrusions, both in traumatized samples (e.g., Gilbertson et al., 2007) and in analogue studies (e.g., Chapter 2). Together, this evidence suggests an important involvement in the initial development of PTSD re-experiencing symptoms. Meanwhile, the emotion regulation strategies cognitive reappraisal and expressive suppression have been linked to PTSD symptoms in traumatized samples (Boden et al., 2013; Eftekhari et al., 2009; Ehring \& Quack, 2010), but data from analogue samples provides only limited support for a link with psychological adjustment (cf. Chapter 4, and the section Affect regulation in the aftermath of emotional challenges above). Thus, the way individuals regulate their emotions appears to moderate the maintenance of PTSD symptoms, but not their initial development. Finally, it seems premature to draw similar conclusions for frontal EEG asymmetry in PTSD, although there are good reasons to expect an involvement in PTSD. That is, Chapter 5 suggests a complex relationship with trauma-potentiated startle, while our literature review in Chapter 6 that only very few studies have tested the involvement of frontal asymmetry in PTSD symptoms.

Following Ehring et al. (2011), the next step would be to devise experimental tests of the causal role of spatial configuration learning, emotion regulation, and frontal asymmetry in PTSD. To date, this step is still missing. Thus, future studies may want to investigate whether it is possible to stimulate or train spatial configuration learning and processing in the hippocampal area, and to test whether this has beneficial consequences in stressful and potentially traumatic experiences. Furthermore, regarding the use of cognitive reappraisal and expressive suppression, it could be possible to establish causal effects by testing the effects of specific emotion regulation trainings on the development and maintenance of PTSD symptoms (see, for a similar proposal, Berking et al., 2008). Finally, next to further establishing links between frontal asymmetry and PTSD symptoms in correlational designs, studies may want to investigate the causal involvement by manipulating frontal asymmetry. 
EEG neurofeedback is a promising and particularly suited tool for this purpose. That is, initial evidence suggests that this method allows enhancing left-sided frontal activity and that this has beneficial effects in individuals with emotional disorders (Baehr, Rosenfeld, \& Baehr, 1997, 2001; Choi et al., 2011).

\section{Concluding remarks}

The present work aimed to advance our understanding of healthy and pathological adjustment to traumatic experiences. A particular focus was on specific memory functions, emotion regulation strategies, and frontal brain asymmetry, i.e., factors that were thought to play a crucial role in the occurrence of involuntary trauma memory, in the subjective and physiological recovery from aversive experiences, and in the development or maintenance of PTSD symptoms. Our results suggest that deficiencies in temporal-lobe based spatial configuration learning contribute to the development intrusive memories following trauma. We found no evidence that this memory function is also involved in negative affect or emotion regulation deficiencies that often accompany intrusions in PTSD. As to the role of emotion regulation strategies, our experimental studies with emotional films lend only very limited support for the view that cognitive reappraisal has universally beneficial effects, and that expressive suppression leads to more harmful outcomes. However, since these strategies have repeatedly been associated with symptom levels in PTSD patients, an appealing interpretation is that these strategies do not cause the development of PTSD symptoms, but rather moderate their persistence.

Next, we presented evidence suggesting that frontal asymmetry - a biomarker that has often been linked to anxiety and depression - is involved in the adjustment to traumatic experiences. In particular, it appears to moderate physiological affect regulation in aversive memories, yet the effects also seem to differ for different types of trauma film. Meanwhile, the literature suggests that resting frontal asymmetry is not robustly related to PTSD symptoms, but provocation-induced EEG asymmetry may be linked with symptoms of this disorder. We recommend more fine-grained analyses into PTSD symptom clusters in relation to frontal asymmetry. Finally, it remains to be seen whether there are qualitative and quantitative differences between PTSD symptoms and the laboratory analogue symptoms that were studied in healthy participants here and elsewhere (Brewin, 2014a; Holmes \& Bourne, 2008). To promote a better integration of these lines of research, we propose that clearer definitions of intrusions, flashbacks, and other instances of involuntary trauma memory will 
be necessary. Finally, this area of research will likely also benefit from integrating various perspectives and methods, including neuropsychological, neuroimaging, and neuroendocrine approaches. 


\section{References}

Aldao, A. (2013). The future of emotion regulation research: Capturing context. Perspectives on Psychological Science, 8, 155-172. doi: 10.1177/1745691612459518

American Psychiatric Association. (2013). Diagnostic and statistical manual of mental disorders (5th ed.). Arlington, VA: American Psychiatric Publishing.

Arntz, A., de Groot, C., \& Kindt, M. (2005). Emotional memory is perceptual. Journal of Behavior Therapy and Experimental Psychiatry, 36, 19-34. doi: 10.1016/j.jbtep.2004.11.003

Baehr, E., Rosenfeld, J. P., \& Baehr, R. (1997). The clinical use of alpha asymmetry biofeedback protocol in treatment of depressive disorders: Two case studies. Journal of Neurotherapy, 2, 12-27.

Baehr, E., Rosenfeld, J. P., \& Baehr, R. (2001). Clinical use of an alpha asymmetry neurofeedback protocol in the treatment of mood disorders: Follow-up study one to five years post therapy. Journal of Neurotherapy, 4, 11-18.

Baird, B., Smallwood, J., Fishman, D. J. F., Mrazek, M. D., \& Schooler, J. W. (2013). Unnoticed intrusions: Dissociations of meta-consciousness in thought suppression. Consciousness and Cognition, 22, 1003-1012. doi: 10.1016/j.concog.2013.06.009

Berking, M., Wupperman, P., Reichardt, A., Pejic, T., Dippel, A., \& Znoj, H. (2008). Emotion-regulation skills as a treatment target in psychotherapy. Behaviour Research and Therapy, 46, 1230-1237. doi: 10.1016/j.brat.2008.08.005

Berntsen, D. (2010). The unbidden past: Involuntary autobiographical memories as a basic mode of remembering. Current Directions in Psychological Science, 19, 138-142.

Bisby, J. A., King, J. A., Brewin, C. R., Burgess, N., \& Curran, H. V. (2010). Acute effects of alcohol on intrusive memory development and viewpoint dependence in spatial memory support a dual representation model. Biological Psychiatry, 68, 280-286.

Boden, M. T., Westermann, S., McRae, K., Kuo, J., Alvarez, J., Kulkarni, M. R., . . BonnMiller, M. O. (2013). Emotion regulation and posttraumatic stress disorder: A prospective investigation. Journal of Social and Clinical Psychology, 32, 296-314.

Bradley, R., Greene, J., Russ, E., Dutra, L., \& Westen, D. (2005). A multidimensional metaanalysis of psychotherapy for PTSD. American Journal of Psychiatry, 162, 214-227. doi: 10.1176/appi.ajp.162.2.214 
Bremner, J. D., Staib, L. H., Kaloupek, D., Southwick, S. M., Soufer, R., \& Charney, D. S. (1999). Neural correlates of exposure to traumatic pictures and sound in Vietnam combat veterans with and without posttraumatic stress disorder: A positron emission tomography study. Biological Psychiatry, 45, 806-816. doi: 10.1016/S00063223(98)00297-2

Brewin, C. R. (2014a). Episodic memory, perceptual memory, and their interaction: Foundations for a theory of posttraumatic stress disorder. Psychological Bulletin, 140, 69-97. doi: 10.1037/a0033722

Brewin, C. R. (2014b). Prospects and problems in studying traumatic flashbacks: Reply to Kvavilashvili (2014). Psychological Bulletin, 140, 105-108. doi: 10.1037/a0034682

Brewin, C. R., Dalgleish, T., \& Joseph, S. (1996). A dual representation theory of posttraumatic stress disorder. Psychological Review, 103, 670-686.

Brewin, C. R., Gregory, J. D., Lipton, M., \& Burgess, N. (2010). Intrusive images in psychological disorders: Characteristics, neural mechanisms, and treatment implications. Psychological Review, 117, 210-232. doi: 10.1037/a0018113

Choi, S. W., Chi, S. E., Chung, S. Y., Kim, J. W., Ahn, C. Y., \& Kim, H. T. (2011). Is alpha wave neurofeedback effective with randomized clinical trials in depression? A pilot study. Neuropsychobiology, 63, 43-51.

Chou, C.-Y., La Marca, R., Steptoe, A., \& Brewin, C. R. (2014a). Biological responses to trauma and the development of intrusive memories: An analog study with the trauma film paradigm. Biological Psychology, 103, 135-143. doi: 10.1016/j.biopsycho.2014.08.002

Chou, C.-Y., La Marca, R., Steptoe, A., \& Brewin, C. R. (2014b). Heart rate, startle response, and intrusive trauma memories. Psychophysiology, 51, 236-246. doi: 10.1111/psyp. 12176

Chua, K. P., \& Chun, M. M. (2003). Implicit scene learning is viewpoint dependent. Perception \& Psychophysics, 65, 72-80.

Chun, M. M., \& Jian, Y. H. (1998). Contextual cueing: Implicit learning and memory of visual context guides spatial attention. Cognitive Psychology, 36, 28-71.

Davidson, R. J. (1998). Affective style and affective disorders: Perspectives from affective neuroscience. Cognition \& Emotion, 12, 307-330.

de Quervain, D. J. F., Aerni, A., Schelling, G., \& Roozendaal, B. (2009). Glucocorticoids and the regulation of memory in health and disease. Frontiers in Neuroendocrinology, 30, 358-370. doi: 10.1016/j.yfrne.2009.03.002 
de Quervain, D. J. F., \& Margraf, J. (2008). Glucocorticoids for the treatment of posttraumatic stress disorder and phobias: A novel therapeutic approach. European Journal of Pharmacology, 583, 365-371. doi: 10.1016/j.ejphar.2007.11.068

Delaney, P. F., Sahakyan, L., Kelley, C. M., \& Zimmerman, C. A. (2010). Remembering to forget: The amnesic effect of daydreaming. Psychological Science, 21, 1036-1042. doi: $10.1177 / 0956797610374739$

Eftekhari, A., Zoellner, L. A., \& Vigil, S. A. (2009). Patterns of emotion regulation and psychopathology. Anxiety Stress and Coping, 22, 571-586. doi: $10.1080 / 10615800802179860$

Ehlers, A. (2010). Understanding and treating unwanted trauma memories in posttraumatic stress disorder. Zeitschrift Fur Psychologie-Journal of Psychology, 218, 141-145. doi: 10.1027/0044-3409/a000021

Ehlers, A., \& Clark, D. M. (2000). A cognitive model of posttraumatic stress disorder. Behaviour Research and Therapy, 38, 319-345. doi: 10.1016/S0005-7967(99)00123-0

Ehring, T., Kleim, B., \& Ehlers, A. (2011). Combining clinical studies and analogue experiments to investigate cognitive mechanisms in posttraumatic stress disorder. International Journal of Cognitive Therapy, 4, 165-177. doi: 10.1521/ijct.2011.4.2.165

Ehring, T., \& Quack, D. (2010). Emotion regulation difficulties in trauma survivors: The role of trauma type and PTSD symptom severity. Behavior Therapy, 41, 587-598. doi: 10.1016/j.beth.2010.04.004

Foa, E. B., Dancu, C. V., Hembree, E. A., Jaycox, L. H., Meadows, E. A., \& Street, G. P. (1999). A comparison of exposure therapy, stress inoculation training, and their combination for reducing posttraumatic stress disorder in female assault victims. Journal of Consulting and Clinical Psychology, 67, 194-200. doi: 10.1037/0022006x.67.2.194

Foa, E. B., \& Kozak, M. J. (1986). Emotional processing of fear: Exposure to corrective information. Psychological Bulletin, 99, 20-35. doi: 10.1037/0033-2909.99.1.20

Foa, E. B., Riggs, D. S., Dancu, C. V., \& Rothbaum, B. O. (1993). Reliability and validity of a brief instrument for assessing post-traumatic stress disorder. Journal of Traumatic Stress, 6, 459-473.

Foti, D., \& Hajcak, G. (2008). Deconstructing reappraisal: Descriptions preceding arousing pictures modulate the subsequent neural response. Journal of Cognitive Neuroscience, 20, 977-988. doi: 10.1162/jocn.2008.20066 
Fyhn, M., Hafting, T., Treves, A., Moser, M.-B., \& Moser, E. I. (2007). Hippocampal remapping and grid realignment in entorhinal cortex. Nature, 446, 190-194.

Galatzer-Levy, I. R., \& Bryant, R. A. (2013). 636,120 ways to have posttraumatic stress disorder. Perspectives on Psychological Science, 8, 651-662. doi: $10.1177 / 1745691613504115$

Garnefski, N., Kraaij, V., \& Spinhoven, P. (2002). Manual for the use of the Cognitive Emotion Regulation Questionnaire. Leiderdorp, The Netherlands: DATEC.

Geschwind, N., Peeters, F., Drukker, M., van Os, J., \& Wichers, M. (2011). Mindfulness training increases momentary positive emotions and reward experience in adults vulnerable to depression: A randomized controlled trial. Journal of Consulting and Clinical Psychology, 79, 618-628. doi: 10.1037/a0024595

Gilbertson, M. W., Williston, S. K., Paulus, L. A., Lasko, N. B., Gurvits, T. V., Shenton, M. E., . . Orr, S. P. (2007). Configural cue performance in identical twins discordant for posttraumatic stress disorder: Theoretical implications for the role of hippocampal function. Biological Psychiatry, 62, 513-520. doi: 10.1016/j.biopsych.2006.12.023

Gross, J. J., \& John, O. P. (2003). Individual differences in two emotion regulation processes: Implications for affect, relationships, and well-being. Journal of Personality and Social Psychology, 85, 348-362. doi: 10.1037/0022-3514.85.2.348

Gross, J. J., \& Thompson, R. A. (2007). Emotion regulation: Conceptual foundations. In J. J. Gross (Ed.), Handbook of emotion regulation (pp. 3-24). New York: The Guilford Press.

Hagenaars, M. A., Brewin, C. R., van Minnen, A., Holmes, E. A., \& Hoogduin, K. A. L. (2010). Intrusive images and intrusive thoughts as different phenomena: Two experimental studies. Memory, 18, 76-84. doi: 10.1080/09658210903476522

Hellawell, S. J., \& Brewin, C. R. (2002). A comparison of flashbacks and ordinary autobiographical memories of trauma: Cognitive resources and behavioural observations. Behaviour Research and Therapy, 40, 1143-1156. doi: 10.1016/s00057967(01)00080-8

Henry, J. D., \& Crawford, J. R. (2005). The short-form version of the Depression Anxiety Stress Scales (DASS-21): Construct validity and normative data in a large non-clinical sample. British Journal of Clinical Psychology, 44, 227-239. doi: $10.1348 / 014466505 \times 29657$ 
Holmes, E. A., \& Bourne, C. (2008). Inducing and modulating intrusive emotional memories: A review of the trauma film paradigm. Acta Psychologica, 127, 553-566. doi: 10.1016/j.actpsy.2007.11.002

Holmes, E. A., James, E. L., Kilford, E. J., \& Deeprose, C. (2010). Key steps in developing a cognitive vaccine against traumatic flashbacks: Visuospatial tetris versus verbal pub quiz. PLoS ONE, 5, e13706. doi: 10.1371/journal.pone.0013706

Holmes, E. A., \& Mathews, A. (2010). Mental imagery in emotion and emotional disorders. Clinical Psychology Review, 30, 349-362. doi: 10.1016/j.cpr.2010.01.001

Hopper, J. W., Frewen, P. A., van der Kolk, B. A., \& Lanius, R. A. (2007). Neural correlates of reexperiencing, avoidance, and dissociation in PTSD: Symptom dimensions and emotion dysregulation in responses to script-driven trauma imagery. Journal of Traumatic Stress, 20, 713-725. doi: 10.1002/jts.20284

Jackson, D. C., Mueller, C. J., Dolski, I., Dalton, K. M., Nitschke, J. B., Urry, H. L., . . . Davidson, R. J. (2003). Now you feel it, now you don't: Frontal brain electrical asymmetry and individual differences in emotion regulation. Psychological Science, $14,612-617$.

Killingsworth, M. A., \& Gilbert, D. T. (2010). A wandering mind is an unhappy mind. Science, 330, 932. doi: 10.1126/science.1192439

Krans, J., Langner, O., Reinecke, A., \& Pearson, D. G. (2013). Intrusive images and voluntary memory for affective pictures: Contextualization and dual-task interference. Journal of Behavior Therapy and Experimental Psychiatry, 44, 418-425.

Krans, J., Naring, G., Holmes, E. A., \& Becker, E. S. (2010). Motion effects on intrusion development. Journal of Trauma and Dissociation, 11, 73-82. doi: $10.1080 / 15299730903318483$

Kuppens, P., Oravecz, Z., \& Tuerlinckx, F. (2010). Feelings change: Accounting for individual differences in the temporal dynamics of affect. Journal of Personality and Social Psychology, 99, 1042-1060. doi: 10.1037/a0020962

Kvavilashvili, L. (2014). Solving the mystery of intrusive flashbacks in posttraumatic stress disorder: Comment on Brewin (2014). Psychological Bulletin, 140, 98-104. doi: $10.1037 / \mathrm{a} 0034677$

Leer, A., Engelhard, I. M., \& van den Hout, M. A. (2014). How eye movements in EMDR work: Changes in memory vividness and emotionality. Journal of Behavior Therapy and Experimental Psychiatry, 45, 396-401. doi: 10.1016/j.jbtep.2014.04.004 
Lovibond, S. H., \& Lovibond, P. F. (1995). Manual for the Depression Anxiety Stress Scales (2nd ed.). Sidney: Psychology Foundation.

McCrae, R. R., \& John, O. P. (1992). An introduction to the 5-factor model and its applications. Journal of Personality, 60, 175-215. doi: 10.1111/j.14676494.1992.tb00970.x

McNally, R. J., Bryant, R. A., \& Ehlers, A. (2003). Does early psychological intervention promote recovery from posttraumatic stress? Psychological Science in the Public Interest, 4, 45-79. doi: 10.1111/1529-1006.01421

Meyer, T., Quaedflieg, C. W. E. M., Giesbrecht, T., Meijer, E., Abiad, S., \& Smeets, T. (2014). Frontal EEG asymmetry as predictor of physiological responses to aversive memories. Psychophysiology, 51, 853-865.

Meyer, T., Smeets, T., Giesbrecht, T., Quaedflieg, C. E. M., Girardelli, M., Mackay, G. N., \& Merckelbach, H. (2013). Individual differences in spatial configuration learning predict the occurrence of intrusive memories. Cognitive, Affective, \& Behavioral Neuroscience, 13, 186-196. doi: 10.3758/s13415-012-0123-9

Moore, S. A., Zoellner, L. A., \& Mollenholt, N. (2008). Are expressive suppression and cognitive reappraisal associated with stress-related symptoms? Behaviour Research and Therapy, 46, 993-1000. doi: 10.1016/j.brat.2008.05.001

Otgaar, H., Scoboria, A., \& Smeets, T. (2013). Experimentally evoking nonbelieved memories for childhood events. Journal of Experimental Psychology: Learning, Memory, and Cognition, 39, 717-730. doi: 10.1037/a0029668

Pearson, D. G., Ross, F. D. C., \& Webster, V. L. (2011). The importance of context: Evidence that contextual representations increase intrusive memories. Journal of Behavior Therapy and Experimental Psychiatry, 43, 573-580.

Preston, A. R., \& Gabrieli, J. D. E. (2008). Dissociation between explicit memory and configural memory in the human medial temporal lobe. Cerebral Cortex, 18, 21922207. doi: 10.1093/cercor/bhm245

Quaedflieg, C. W. E. M., Schwabe, L., Meyer, T., \& Smeets, T. (2013). Time dependent effects of stress prior to encoding on event-related potentials and $24 \mathrm{~h}$ delayed retrieval. Psychoneuroendocrinology, 38, 3057-3069. doi: 10.1016/j.psyneuen.2013.09.002

Rabe, S., Beauducel, A., Zöllner, T., Maercker, A., \& Karl, A. (2006). Regional brain electrical activity in posttraumatic stress disorder after motor vehicle accident. Journal of Abnormal Psychology, 115, 687-698. doi: 10.1037/0021-843x.115.4.687 
Rabe, S., Zöllner, T., Beauducel, A., Maercker, A., \& Karl, A. (2008). Changes in brain electrical activity after cognitive behavioral therapy for posttraumatic stress disorder in patients injured in motor vehicle accidents. Psychosomatic Medicine, 70, 13-19. doi: 10.1097/PSY.0b013e31815aa325

Robison-Andrew, E. J., Duval, E. R., Nelson, C. B., Echiverri-Cohen, A., Giardino, N., Defever, A., . . Rauch, S. A. M. (2014). Changes in trauma-potentiated startle with treatment of posttraumatic stress disorder in combat Veterans. Journal of Anxiety Disorders, 28, 358-362. doi: 10.1016/j.janxdis.2014.04.002

Rose, S., Bisson, J., \& Wessely, S. (2003). A systematic review of single-session psychological interventions ('debriefing') following trauma. Psychotherapy and Psychosomatics, 72, 176-184.

Rubin, D. C., Berntsen, D., \& Bohni, M. K. (2008). Memory-based model of posttraumatic stress disorder: Evaluating basic assumptions underlying the PTSD diagnosis. Psychological Review, 115, 985-1011. doi: 10.1037/a0013397

Schooler, J. W. (2002). Re-representing consciousness: Dissociations between experience and meta-consciousness. Trends in Cognitive Sciences, 6, 339-344. doi: 10.1016/S13646613(02)01949-6

Schwabe, L., Wolf, O. T., \& Oitzl, M. S. (2010). Memory formation under stress: Quantity and quality. Neuroscience \& Biobehavioral Reviews, 34, 584-591. doi: 10.1016/j.neubiorev.2009.11.015

Scoboria, A., Mazzoni, G., Kirsch, I., \& Relyea, M. (2004). Plausibility and belief in autobiographical memory. Applied Cognitive Psychology, 18, 791-807. doi: 10.1002/acp.1062

Smallwood, J., \& Schooler, J. W. (2015). The science of mindwandering: Empirically navigating the stream of consciousness. Annual Review of Psychology, 66, 487-518. doi: 10.1146/annurev-psych-010814-015331

Smeets, T. (2011). Acute stress impairs memory retrieval independent of time of day. Psychoneuroendocrinology, 36, 495-501.

Smeets, T., Merckelbach, H., Horselenberg, R., \& Jelicic, M. (2005). Trying to recollect past events: Confidence, beliefs, and memories. Clinical Psychology Review, 25, 917-934. doi: 10.1016/j.cpr.2005.03.005

Smeets, T., Otgaar, H., Candel, I., \& Wolf, O. T. (2008). True or false? Memory is differentially affected by stress-induced cortisol elevations and sympathetic activity at 
consolidation and retrieval. Psychoneuroendocrinology, 33, 1378-1386. doi: 10.1016/j.psyneuen.2008.07.009

Soravia, L. M., Heinrichs, M., Aerni, A., Maroni, C., Schelling, G., Ehlert, U., . . . de Quervain, D. J.-F. (2006). Glucocorticoids reduce phobic fear in humans. Proceedings of the National Academy of Sciences, 103, 5585-5590. doi: 10.1073/pnas.0509184103

Takarangi, M. K., Strange, D., \& Lindsay, D. S. (2014). Self-report may underestimate trauma intrusions. Consciousness and Cognition, 27, 297-305.

Taverniers, J., Smeets, T., Lo Bue, S., Syroit, J., Van Ruysseveldt, J., Pattyn, N., \& von Grumbkow, J. (2011). Visuo-spatial path learning, stress, and cortisol secretion following military cadets' first parachute jump: the effect of increasing task complexity. Cognitive, Affective, \& Behavioral Neuroscience, 11, 332-343. doi: 10.3758/s13415-011-0043-0

Tryon, W. W. (2005). Possible mechanisms for why desensitization and exposure therapy work. Clinical Psychology Review, 25, 67-95. doi: 10.1016/j.cpr.2004.08.005

van Ast, V. A., Cornelisse, S., Meeter, M., Joëls, M., \& Kindt, M. (2013). Time-dependent effects of cortisol on the contextualization of emotional memories. Biological Psychiatry, 74, 809-816. doi: 10.1016/j.biopsych.2013.06.022

Watson, D., Clark, L. A., \& Tellegen, A. (1988). Development and validation of brief measures of positive and negative affect: The PANAS scales. Journal of Personality and Social Psychology, 54, 1063-1070.

Wingenfeld, K., \& Wolf, O. T. (2011). HPA axis alterations in mental disorders: Impact on memory and its relevance for therapeutic interventions. CNS Neuroscience \& Therapeutics, 17, 714-722. doi: 10.1111/j.1755-5949.2010.00207.x

Wolf, O. T. (2008). The influence of stress hormones on emotional memory: Relevance for psychopathology. Acta Psychologica, 127, 513-531. doi: 10.1016/j.actpsy.2007.08.002 

Summary 



\section{Summary}

The work presented in this dissertation aimed to advance our understanding of healthy and pathological adjustment to traumatic experiences. A particular focus was on specific memory functions, emotion regulation strategies, and frontal brain asymmetry, as factors that may play a crucial role in the occurrence of involuntary trauma memory, in the subjective and physiological recovery from aversive experiences, and in the development or maintenance of post-traumatic stress disorder (PTSD) symptoms. In particular, Chapter 2 tested whether spatial configuration learning, a memory function known to critically depend on the hippocampal area of the brain, would predict PTSD-like symptoms following exposure to a trauma film. Indeed, better performance predicted fewer intrusions, but not the distress caused by these intrusions, nor other PTSD symptoms. In addition, Chapter 3 found that acute stress modulates this particular memory function, but differently for individuals with high versus low endogenous cortisol secretion in response to stress. That is, performance was only impaired under stress for participants with low cortisol secretion, suggesting a protective role of the hormonal response.

Next, Chapter 4 investigated the role of specific emotion regulation strategies in daily life and following emotional provocation. Cognitive reappraisal, which influences emotion by changing cognitions about a given situation, appeared to reduce only the arousal component of emotion and only under certain contextual circumstances. Meanwhile, expressive suppression, a strategy targeting bodily responses associated with an emotion, was entirely unrelated to diurnal affect and recovery from a laboratory fear induction procedure. Additional analyses (see General Discussion) indicated that cognitive reappraisal and expressive suppression exert very little (if any) influence on PTSD analogue symptoms in two studies using the trauma-film paradigm. The relatively limited role of these strategies in our laboratory studies contradicts recurrent findings in trauma victims, according to which PTSD is accompanied by higher levels of cognitive reappraisal and lower levels of expressive suppression. A reason for this discrepancy might be that these emotion regulation strategies are unrelated to the onset of PTSD symptoms, but play a role in their maintenance.

Chapter 5 focused on frontal asymmetry, an electroencephalography (EEG) based biomarker in relation to memory-potentiated startle responses following exposure to a trauma film. We expected left-sided frontal activity to be associated with adaptive emotion regulation and hence to predict better physiological self-regulation in response to reminders of a trauma film. In line with this, more left-sided frontal activation during film viewing predicted 
dampened startle responses following the offset of reminder pictures, but only if individuals had seen a staged road accident. For participants who had viewed a genocide documentary, this effect tended to be reversed. In a second study, we manipulated the degree to which participants used positive reappraisal in response to the genocide film. However, this did not moderate the effects of frontal asymmetry, with our results showing that left frontal activity at rest predicted higher startle responses. This highlights the need for further critical investigation to understand the involvement of this biomarker in memory-potentiated startle. On the one hand, frontal asymmetry may play opposing roles in different types of provocation. Alternatively, in the general discussion, we issued the idea that adaptive physiological self-regulation may require more and longer emotional processing in response to the genocide film, compared to the road accident film. Therefore, not the role of frontal asymmetry, but the functional significance of sustained memory-potentiated startle may have differed between the two film conditions.

$\underline{\text { Chapter } 6}$ reviewed the frontal asymmetry literature, where studies have typically found that more left-sided frontal activity is related to approach motivation and lower levels of depression and anxiety. A similar relation can thus be expected with PTSD, because this disorder shares dysphoria- and fear-related symptoms with depression and anxiety. In addition, several lines of research provide plausible links between functional asymmetries in the brain and the neuropsychological abnormalities that characterize PTSD. However, neuroimaging studies rarely perform explicit tests of laterality effects, and only eight studies directly tested the association between frontal EEG asymmetry and PTSD. These studies provide almost no support for a role of resting frontal EEG asymmetry in this disorder. However, state-induced asymmetry during trauma-relevant stimulation might distinguish trauma victims with and without PTSD. Chapter 6 concludes by entertaining hypotheses that may guide future research and help to fully apprehend the practical and theoretical relevance of this biomarker. Importantly, frontal asymmetry could be a transdiagnostic marker of psychological adjustment, and we suggest investigating its relationship with more specific symptom clusters rather than diagnostic status of PTSD.

Finally, Chapter 7 (general discussion) provides a summary of the most important findings, addresses their key implications, and suggests promising routes for future research. For instance, we propose that it is worthwhile to further study the involvement of hippocampal-area based spatial learning in PTSD and the interaction with other memory systems and neuroendocrine stress responses, which may also contribute to improved psychological interventions targeting flashbacks in trauma victims. Furthermore, the chapter 
presents previously unpublished data that help to clarify the roles of cognitive reappraisal, expressive suppression, and frontal EEG asymmetry in psychological adjustment following stress and trauma. Next, this chapter discusses how experimental studies in healthy participants and studies in trauma-exposed samples can jointly advance our understanding of psychological adjustment to stress and trauma. We advocate the use of clearer and stricter definitions for interrelated concepts such as involuntary memories, intrusions, flashbacks, or trauma-related mind-wandering, because there may be qualitative and quantitative differences between these phenomena that are measured in analogue versus clinical traumatised samples. The chapter concludes by highlighting the current state of knowledge regarding the adaptive functions of spatial memory formation in the hippocampal area, emotion regulation by means of reappraisal and expressive suppression, and frontal EEG asymmetry, following exposure to stress and trauma. 

Samenvatting 



\section{Samenvatting}

Het doel van dit proefschrift was om meer inzicht te verkrijgen in de vraag waarom sommige mensen beter met traumatische ervaringen kunnen omgaan dan anderen. Een bijzondere nadruk lag hierbij op individuele verschillen in specifieke geheugenfuncties, emotieregulatie strategieën en zogenaamde frontale asymmetrie in de hersenen. Het idee was dat deze factoren bepalen hoe mensen subjectief en fysiologisch van aversieve ervaringen herstellen, en dat ze een cruciale rol spelen bij het ontstaan van een posttraumatische stressstoornis (PTSS). Deze stoornis is, onder meer, gekenmerkt door onvrijwillige, steeds weer terugkerende, traumatische herinneringen. Specifiek heeft $\underline{\text { Hoofdstuk } 2}$ onderzocht of het leren van ruimtelijke configuraties, een geheugenfunctie waarbij het hippocampale gebied in de hersenen van essentieel belang is, voorspelt of mensen na het zien van een schokkende film PTSS-achtige klachten zouden vertonen. Wij vonden dat zulks inderdaad het geval was: een betere leerprestatie ging gepaard met minder ongewilde herinneringen. Er was echter geen samenhang met andere PTSS klachten. In $\underline{\text { Hoofdstuk } 3}$ vonden we bovendien dat het leren van ruimtelijke configuraties door acute stress wordt beïnvloedt. Het effect van stress hing echter ervan af of mensen veel of weinig van het stresshormoon cortisol afscheidden. Enkel bij deelnemers met een lage cortisol respons leidde stress tot verslechterde leerprestatie, hetgeen suggereert dat cortisol afscheiding tijdens stress een beschermende rol speelt. Vervolgens heeft $\underline{\text { Hoofdstuk } 4}$ onderzocht welke rol bepaalde emotieregulatie strategieën in het dagelijkse leven en na een emotionele provocatie uitoefenen. We vonden dat het herwaarderen van emotionele situaties ("cognitive reappraisal") enkel het arousal component van emotie lijkt te dempen, en dan nog alleen maar onder bepaalde omstandigheden. Verder vonden we dat mensen die het uiten van hun emoties vaker onderdrukken ("expressive suppression") niet van andere mensen verschillen in de alledaagse emotionele beleving. Ook herstelden deze personen niet meer of minder goed van de emotionele provocatie. Extra analyses (zie daartoe de algemene discussie) lieten verder zien dat reappraisal en suppression maar heel weinig invloed hebben op PTSS-achtige klachten na het zien van schokkende films. Dit lijkt literatuur waarin patiënten met PTSS vaak te weinig reappraisal en teveel suppression rapporteren tegen te spreken. Een mogelijke verklaring voor deze discrepantie is dat deze strategieën een rol spelen bij de instandhouding, maar niet het ontstaan van PTSS. $\underline{\text { Hoofdstuk }}$ $\underline{5}$ keek naar frontale hersenasymmetrie, een biomarker die met elektroencefalografie gemeten wordt, als mogelijke voorspeller van schrikresponses bij deelnemers die van tevoren een trauma film gezien hebben. We verwachtten dat hogere linkszijdige activiteit met betere 
emotieregulatie gepaard zou gaan, en dus zou samenhangen met betere fysiologische zelfregulatie na het zien van plaatjes van de trauma film. Dit patroon vonden wij inderdaad, echter alleen bij proefpersonen die een educatieve film van een zwaar auto-ongeluk gezien hadden. Bij proefpersonen die een documentaire over een genocide hebben bleek dit effect zelfs tegenovergesteld. In een vervolgstudie hebben we de mate waarin deelnemers positief over de genocide film gingen denken ("positive reappraisal”) gemanipuleerd. Dit had echter geen invloed op de effecten van frontale asymmetrie: meer linkszijdige activiteit voorspelde hogere schrikreacties. Deze bevindingen benadrukken het belang van verder onderzoek naar de rol van deze biomarker in geheugen-gerelateerde schrikresponses: het zou kunnen dat frontale asymmetrie bij verschillende provocaties tegenovergestelde effecten heeft. Een alternatieve verklaring, dewelke we in de discussie bespreken, is dat adaptieve fysiologische zelfregulatie bij de genocide film intensievere emotionele verwerking vereist dan de film over een auto-ongeluk. Dit zou betekenen dat niet de rol van frontale asymmetrie, maar die van geheugen-gerelateerde schrikresponses tussen de film condities verschilt.

$\underline{\text { Hoofdstuk } 6}$ verschaft een overzicht van de frontale asymmetrie literatuur. Hieruit blijkt dat linkszijdige activiteit vaak gepaard gaat met een hogere toenaderingsmotivatie en met minder depressie en angstklachten. Gezien PTSS ook fobische en stemming gerelateerde klachten omvat, zou dus ook deze stoornis door hogere rechtszijdige activiteit gekenmerkt kunnen zijn. Daarnaast bestaan er plausibele verbanden tussen functionele asymmetriëen in de hersenen en het patroon van abnormale hersenactiviteit dat PTSS patiënten vaak laten zien. Expliciete tests van asymmetrische effecten worden echter zelden uitgevoerd, en slechts acht studies hebben het verband tussen EEG asymmetrie en PTSS direct onderzocht. Hieruit blijkt weinig evidentie voor de assumptie dat frontale asymmetrie tijdens rust met PTSS-achtige symptomen samenhangt. Wel zijn er aanwijzingen dat meer rechtszijdige frontale activatie tijdens trauma-gerelateerde stimulatie indicatief voor PTSS kan zijn. Hoofdstuk 6 sluit af met nieuwe hypotheses, welke toekomstig onderzoek op dit gebied kunnen inspireren en kunnen helpen de praktische en theoretische relevantie van deze biomarker beter te begrijpen. Wij opperen dat frontale asymmetrie mogelijk als transdiagnostische marker van psychologische aanpassing kan fungeren, en dat toekomstig onderzoek zich beter op de rol van deze marker in specifieke symptoomclusters kan concentreren in plaats van op de diagnose PTSS.

Als afsluiting van dit proefschrift geeft Hoofdstuk 7 (algemene discussie) een samenvatting van de bevindingen, bespreekt de belangrijkste implicaties, evenals mogelijke routes voor toekomstig onderzoek. Onder meer bevelen we aan om nader te onderzoeken welke rol ruimtelijke informatieopslag in het hippocampale gebied bij PTSS speelt, en in 
hoeverre dit effect van andere geheugensystemen en hormonale stress responses afhangt. Wij denken dat dit onderzoek aan de verbetering van psychologische interventies tegen traumatische flashbacks kan bijdragen. Verder presenteren wij in dit hoofdstuk ook ongepubliceerde data die de rol van cognitive reappraisal, het onderdrukken van emoties, en frontale asymmetrie na het meemaken van een traumatische ervaring zouden kunnen verhelderen. Vervolgens bespreken wij hoe experimenteel onderzoek bij gezonde proefpersonen en studies met getraumatiseerde deelnemers gezamenlijk kunnen helpen om meer inzicht te krijgen in psychologische aanpassing aan stress en trauma's. Daarbij is onze aanbeveling om duidelijkere en striktere definities te gebruiken om trauma-gerelateerde concepten te beschrijven, zoals ongewilde herinneringen, intrusies, flashbacks, of het afdwalen van gedachtes. Wij argumenteren dat er kwantitatieve en kwalitatieve verschillen tussen deze fenomenen kunnen bestaan. Afsluitend vat dit hoofdstuk de huidige stand van zaken samen met betrekking tot de adaptieve rol van ruimtelijke geheugenvorming in het hippocampale gebied, emotieregulatie door middel van cognitive reappraisal of expressive suppression, en frontale hersenasymmetrie, na het meemaken van stress en trauma. 

Valorisation Addendum 



\section{Valorisation Addendum}

\section{Relevance}

Many people are exposed to severe negative events in their life, and there are large individual differences in how people respond to and recover from such events. The present work was inspired by the question why some people appear to be resilient to aversive experiences, whereas others develop and maintain pathological symptoms afterwards. An underlying idea is that it may be possible to link resilience to measurable individual characteristics - which could eventually be used to make prognoses, monitor treatment outcomes, develop novel treatment interventions, and more generally, to better understand trauma-related psychopathology. As well, understanding the individual characteristics that are associated with resilience would enable us to predict psychological adjustment even before the occurrence of a traumatic event. This knowledge could thus be used to help prevent posttraumatic stress disorder (PTSD) in individuals with a high risk of trauma exposure (e.g., fire fighters, military personnel, police officers), potentially by developing individually tailored resilience trainings. With this in mind, the chapters of the current dissertation focused on various person characteristics as potential predictors of emotional adjustment, particularly following negative and stressful experiences. The characteristics that we studied can be grouped into factors relating to memory formation on the one hand (Chapters 2 and 3), and to the regulation of emotion, on the other (Chapters $4-6$ ). 


\section{To whom are the research results of interest?}

It is now well established that people differ in their response to stress and trauma, which leads to the question of what determines the course of psychological adjustment and resilience. This dissertation aimed to contribute to answering this question and may thus be of interest to a broader public of researchers and clinicians who strive for a better understanding of psychological adjustment to aversive experiences, factors that predict the development of post-traumatic stress disorder, and more generally, resilience to psychopathology. Primarily, the presented work targets members of the scientific community working in various fields including emotion, memory, and affective neuroscience. Secondarily, it may be of interest to clinicians and researchers who are working to bridge existing gaps between research and theory on the one hand, and clinical practice on the other. 


\section{Potential products, services, processes, or activities}

This dissertation contributes to the search for factors that can predict psychological adjustment to trauma. This search is fuelled by the ambition to understand and help prevent the development of PTSD more effectively. Eventually, the insights gained by this line of research might be used to improve the diagnostic process and facilitate prognoses, monitor treatment outcomes, develop novel treatment interventions as well as resilience trainings, and more generally, to validate clinical theories of PTSD for a better understanding of traumarelated psychopathology. Notably, the development of PTSD following a traumatic experience may not be attributable to a single mechanism (see, e.g., Chapter 1). In line with this, the findings presented in the various chapters of this dissertation indicate that individual factors such as memory formation in the hippocampal area, cognitive emotion regulation, or asymmetric frontal brain activity may be involved in the psychological adjustment to aversive experiences, but taken individually, they may not serve as robust predictors of the entire clinical picture presented by PTSD patients. These insights should encourage and inform efforts to develop products, services, or activities, such as preventive or treatment interventions aiming to foster specific memory functions, train the use of emotion regulation strategies, or alter patterns of brain asymmetry (e.g., neurofeedback, electrical stimulation). 


\section{Innovation}

In the past decades, research on the predictive factors of PTSD has advanced rapidly and has provided many novel insights into risk and resilience factors. Jointly, the evidence suggests that psychological adjustment to trauma is related to individual differences in various psychological and psychophysiological factors before, during, and after the traumatic event. This dissertation makes innovative contributions to these research efforts by empirically testing a set of assumptions about the role of memory, emotion regulation, and frontal brain asymmetry in the psychological adjustment following aversive experiences. In particular, we tested whether temporal-lobe-based memory formation is related to intrusions following aversive experiences. As well, we investigated how acute stress and stress hormones alter the efficiency of this memory formation. Furthermore, we looked into the role of specific emotion regulation strategies in emotional recovery from a provocation and in everyday affective experiences. Next, we addressed a possible involvement of frontal asymmetry in the emotional adjustment to aversive experiences, specifically by looking at physiological selfregulation in response to reactivation of aversive memories, and by reviewing empirical links with PTSD. Finally, we refer the interested reader to the general discussion (Chapter 7), where we highlight several key theoretical and practical implications and indicate promising avenues for future research that flow from the work presented here. 


\section{Schedule and implementation}

The work presented in this dissertation aimed to advance our understanding of why some people appear to be resilient to aversive experiences, whereas others develop and maintain pathological symptoms afterwards. That is, this dissertation focuses on knowledge acquisition with respect to a relatively fundamental research question. Therefore, its contribution to the scheduling and implementation of specific products, services, processes, or activities is an indirect one. As outlined above, our findings may well prove to contribute to the development of tools to make prognoses, monitor treatment outcomes, or to develop novel preventive and treatment interventions for trauma victims or individuals who are at high risk to be exposed to trauma. This could entail preventive or treatment interventions aiming to foster specific memory functions, train the use of emotion regulation strategies, or alter patterns of brain asymmetry. Rather than having immediate relevance for such practical implementations, note that the findings of this dissertation suggest interesting routes for future research, as outlined in the discussion sections of Chapters $2-6$, and as elaborated in the general discussion (Chapter 7). Importantly, Chapter 7 emphasizes that our empirical findings are based on studies in healthy participants, and that these analogue studies should be complemented by research in traumatised individuals before the gained knowledge can be implemented in that latter population. Only thereby, the involvement of specific factors in the psychological adjustment to stress and trauma, as well as in the development of PTSD, can be fully evaluated. Furthermore, this dissertation focused on the establishment of statistical associations between candidate factors and outcome measures related to psychological adjustment, including PTSD analogue symptoms. An important next step would be to establish whether these factors are causally involved in PTSD. Thus, our findings can inform future research that devises experimental tests of the causal role of spatial configuration learning, emotion regulation, and frontal asymmetry in PTSD. These studies may want to investigate whether it is possible to stimulate or train spatial configuration learning and processing in the hippocampal area, and whether this has beneficial consequences following trauma exposure. Regarding the use of specific emotion regulation strategies, causal effects could by tested by employing emotion regulation trainings. Finally, studies may want to investigate the causal involvement of frontal asymmetry in the adjustment to stress and trauma, for instance by using EEG neurofeedback. 

Acknowledgements 



\section{Acknowledgements}

I would like to thank all those who have supported and accompanied me and who have shared experiences with me during the last five years. Only pleasant memories come to mind when I think about my PhD years. I remember a friendly, collaborative, and stimulating environment in my section, department, and faculty. As well, I remember many great moments, enjoyable routines, and the comfort of feeling at home in Maastricht, Liège, Aachen, and not to forget, in UNS40. It was a wonderful time.

Special thanks are due to my supervisors. First of all, I thank you, Tom, for supporting and making this project possible on a daily basis. It was great to work with you and having you as a mentor - thanks for so much trust, freedom, support, advice, and kindness. You were practically always available, ready to help with anything that would come up, and ready to give thorough feedback in no time. That was quite a luxury. I really hope that we will continue to work together. Luckily, I left enough data in your office cupboard that waits to be written up, so I will gladly be in touch. Also Harald, thanks for your patience and appreciation of my work, and for sharing your enthusiasm and wisdom. Timo, I really appreciated your input and availability, even from a distance. The meetings we had from time to time that were always full of critical feedback, practical solutions, and good advice.

Next, I wish to thank my fantastic colleagues. Conny, you were an important part of this $\mathrm{PhD}$ project and experience. We were a great team, and quite a lot would have been different without you. Next to your valuable input, I appreciated your super-efficiency, organization skills, determination, and assertiveness. I have learned from that. I enjoyed working with you and hope to do so in the future. And of course, now I'm glad to have your moral support as a paranymph. Thanks also to my wonderful roommates - you made it a pleasure to come to work every day. I'll miss exchanging ideas and our discussions of everyday issues, news, taste, lifestyle, and music, as well as your presence when we were eagerly working. Dear lunch, diner, and coffee loving fellows, and dear weekend snerds, thanks for your enjoyable company that I will surely miss. Marjolein, thanks for the inspiration that resulted in the cover of this book.

To all my (past and future) co-authors and collaborators, thanks for your critical input, from which I have learned a lot, and for sharing your expertise and enthusiasm. I am especially grateful for all inspirational exchanges of ideas that have resulted in exciting plans and real projects. To the always helpful instrumentation guys, thank you for your patience with my paradigms and for making everything run smoothly. And when I was looking for 
motivation at the coffee machine, thanks for reminding me that, at least, it was almost weekend. Dear students whom I had the pleasure to work with, thanks for your enthusiasm and your help in implementing and conducting the studies. Surely, this dissertation would have been shorter without your contributions.

A big thank you goes to all those who have been close to me and who shared or accompanied my Maastricht experience, including my parents, friends, and colleagues. Felix, since we met at the language course in 2004, you have played an important role in my development throughout the studies and later on. Thanks for a lot of valuable advice, things I have learned from you, and for all we shared studying, as housemates, cycling thousands of kilometres, and then as $\mathrm{PhD}$ students. Who knows whether I would be where I am now if I had not known you. Gesa, you are another constant factor that belongs to life in Maastricht. I appreciate your calm, supportive, and accepting nature. Therefore, it is wonderful to have your backing as my paranymph. Thanks also to all others who have become friends along the way, some still holding the line in Maastricht and others who are now scattered all around the world. Let's keep in touch.

Finally, I thank Alice, who has been the closest person to me throughout these years. Thank you for your support, interest, loyalty, for a lot of patience, and for a lot of positive emotion. I will always remember the nice things we shared with a smile. 


\section{Curriculum Vitae}





\section{Curriculum Vitae}

Thomas Meyer was born April 24, 1984 in Schwerin, Germany. In 1993, he moved with his family to Aachen, where he completed secondary education in 2003 and received the German university-entrance diploma Abitur from the Einhard-Gymnasium. He then did an alternative civilian service at WABe e.V. Wärmestube in Aachen, a day care for people experiencing poverty and/or difficult social situations. In 2004, he started studying psychology at the Faculty of Psychology and Neuroscience at Maastricht University. Between 2005 and 2009, he served as a student member of the faculty council for three academic years. In 2006, he completed the faculty's honours programme. In 2007, he did an Erasmus exchange semester at Catholic University of the Sacred Heart in Milan, Italy. In the same year, he received his Bachelor's degree in Biological Psychology and enrolled in the psychopathology track of the research master Cognitive Neuroscience, Neuropsychology and Psychopathology at Maastricht University. As a part of this programme, he performed a clinical psychology internship at the University Hospital Aachen. After receiving his Master's degree cum laude in 2009, he worked for five years as a PhD candidate at the department of Clinical Psychological Science, section Forensic Psychology, Maastricht University. Since 2015, he holds a postdoc position at the Behavioural Science Institute of Radboud University Nijmegen. 


\section{Publications}

\section{Journal articles}

Meyer, T., Smeets, T., Giesbrecht, T., Quaedflieg, C. W. E. M., Smulders, F., Meijer, E., \& Merckelbach, H. (in press). The role of frontal EEG asymmetry in post-traumatic stress disorder. Biological Psychology.

Quaedflieg, C. W. E. M., van de Ven, V., Meyer, T., Siep, N., Merckelbach, H., \& Smeets, T. (in press). Temporal dynamics of stress-induced alternations of intrinsic amygdala connectivity and neuroendocrine levels. PloS ONE.

Meyer, T., Otgaar, H., \& Smeets, T. (2015). Flashbacks, intrusions, mind-wandering Instances of an involuntary memory spectrum: A commentary on Takarangi, Strange, and Lindsay (2014). Consciousness and Cognition, 33, 24-29.

Quaedflieg, C. W. E. M., Meyer, T., Smulders, F., Merckelbach, H., \& Smeets, T. (2015). The functional role of asymmetric individual-alpha based frontal asymmetry in stress responding. Biological Psychology, 104, 75-81.

Meyer, T., Quaedflieg, C. W. E. M., Giesbrecht, T., Meijer, E., Abiad, S., \& Smeets, T. (2014). Frontal EEG asymmetry as predictor of physiological responses to aversive memories. Psychophysiology, 51, 853-865.

Meyer, T., Smeets, T., Giesbrecht, T., Quaedflieg, C. W. E. M., Girardelli, M., Mackay, G., \& Merckelbach, H. (2013). Individual differences in spatial configuration learning predict the occurrence of intrusive memories. Cognitive, Affective, \& Behavioral Neuroscience, 13, 186-196.

Meyer, T., Smeets, T., Giesbrecht, T., Quaedflieg, C. W. E. M., \& Merckelbach, H. (2013). Acute stress differentially affects spatial configuration learning in high and low cortisol responding healthy adults. European Journal of Psychotraumatology, 4.

Quaedflieg, C. W. E. M., Meyer, T., \& Smeets, T. (2013). The imaging Maastricht Acute Stress Test (iMAST): A neuroimaging compatible psychophysiological stressor. Psychophysiology, 50, 758-766.

Quaedflieg, C. W. E. M., Schwabe, L., Meyer, T., \& Smeets, T. (2013). Time dependent effects of encoding stress on processing efficiency and $24 \mathrm{~h}$ delayed retrieval. Psychoneuroendocrinology, 38, 3057-3069. 
Meyer, T., Smeets, T., Giesbrecht, T., \& Merckelbach, H. (2012). The efficiency of reappraisal and expressive suppression in regulating everyday affective experiences. Psychiatry Research, 200, 964-969.

Smeets, T., Cornelisse, S., Quaedflieg, C. W. E. M., Meyer, T., Jelicic, M., \& Merckelbach, H. (2012). Introducing the Maastricht Acute Stress Test (MAST): A quick and noninvasive approach to elicit robust autonomic and glucocorticoid stress responses. Psychoneuroendocrinology, 37, 1998-2008.

\section{Submitted manuscripts}

Merckelbach, H., Giesbrecht, T., van der Kloet, D., De Jong, J., Meyer, T., \& Rietman, K. (under review). The overlap between dissociative symptoms and symptom overreporting.

Quaedflieg, C. W. E. M., Smulders, F., Meyer, T., Giesbrecht, T., Merckelbach, H. \& Smeets, T. (under review). The validity of individual frontal alpha asymmetry EEG neurofeedback in targeting stress resilience.

\section{Conference presentations}

Meyer, T., Quaedflieg, C. W. E. M., \& Smeets, T. (2015). The relation between visual memory contextualization and trauma-film intrusions. Paper presented at the inaugural International Convention of Psychological Science, Amsterdam, The Netherlands.

Schreuder, M., \& Meyer, T. (2014). Earwitness speaker identification and physiological stress responses. Paper presented at the annual conference of the International Association for Forensic Phonetics and Acoustics, Zürich, Switzerland.

Quaedflieg, C. W. E. M., van de Ven, V., Meyer, T., Siep, N., Merckelbach, H., \& Smeets, T. (2014). Intrinsic amygdala network changes with neuroendocrine levels in acute stress and recovery. Paper presented at the annual convention of the Association for Psychological Science, San Francisco, USA.

Meyer, T. (2013). The role of frontal brain asymmetry in physiological responding to negative memories. Paper presented at the $13^{\text {th }}$ conference of the European Society for Traumatic Stress Studies, Bologna, Italy. 
Quaedflieg, C. W. E. M., \& Meyer, T. (2013). Learning to respond adaptively to stress using EEG neurofeedback. Workshop given at the $2^{\text {nd }}$ Forensic Psychology Update, Maastricht, The Netherlands.

Meyer, T. (2012). Spatial configuration learning and resilience after trauma. Paper presented at masterclass 'State of the art in Trauma and PTSD - From research to practice' by Arq Psychotrauma Expert Group, Amsterdam, The Netherlands.

Meyer, T., Smeets, T., Giesbrecht, T., \& Merckelbach, H. (2011). Individual differences in hippocampal configuration learning predict the occurrence of intrusive memories. Paper presented at the Society of Applied Neuroscience (SAN) meeting, Thessaloniki, Greece. 